MODELLING HUMAN

BEHAVIOUR IN

COUPLED HUMAN- AND

NATURAL SYSTEMS

Koen de Koning 


\section{Modelling Human Behaviour in Coupled Human and Natural Systems}

Koen de Koning 

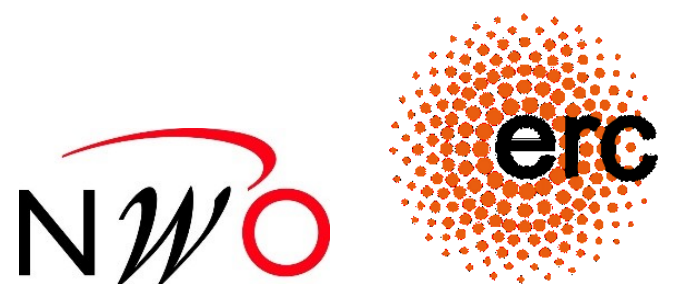

\section{UNIVERSITY OF TWENTE.}

This work was supported by The Netherlands Organisation of Scientific Research (NWO) VENI Grant (No 45I-I I-033), the European Research Council (ERC) under the European Union's Horizon 2020 research and innovation programme (grant agreement No 7580 I4 SCALAR), and by funding from the Faculty of Behavioural, Management and Social sciences, University of Twente.

Cover design: Tim de Koning

Published by: Gildeprint Enschede

ISBN: 978-94-632-3554-9

DOI: $10.3990 / 1.9789463235549$

Copyright (C) Koen de Koning, 2019, Enschede, the Netherlands

All rights reserved. No parts of this thesis may be reproduced, stored in a retrieval system or transmitted in any form or by any means without permission of the author. 


\title{
MODELLING HUMAN BEHAVIOUR IN COUPLED HUMAN AND NATURAL SYSTEMS
}

\author{
DISSERTATION \\ to obtain \\ the degree of doctor at the University of Twente, \\ on the authority of the rector magnificus, \\ prof. dr. T.T.M. Palstra, \\ on account of the decision of the graduation committee, \\ to be publicly defended \\ on Thursday the $4^{\text {th }}$ of April 2019 at 14:45 hrs
}

by

Koen de Koning

born on the $25^{\text {th }}$ of July 1991

in Venlo, the Netherlands 
This dissertation has been approved by:

Prof. dr. T. Filatova

Prof. dr. A. Need

\section{Graduation Committee:}

Chair

Prof. dr. T.A.J. Toonen University of Twente

Pomotor

Prof. dr. T. Filatova

University of Twente

Co-promotor

Prof. dr. A. Need University of Twente

Members

Prof. dr. J. van Hillegersberg University of Twente

Prof. dr. ir. B.P. Veldkamp University of Twente

Prof. dr. ir. G.J. Hofstede Wageningen University and Research

Prof. dr. V. Grimm Helmholz Centre for Envioronmenal Research

Dr. N. Schwarz University of Twente

Dr. J.G. Polhill James Hutton Institute

Dr. W. Jager University of Groningen 


\section{Acknowledgements}

This is it then. This book is the rewarding result of four years of hard work. A PhD can be from time to time a lonely struggle. But luckily I did not have to struggle all on my own. I managed to accomplish this work thanks to great support from my supervisors, colleagues, family, friends and other people who inspired me along the way. I would like to take this opportunity to thank those who played a significant role in this part of my life and those who helped me get where I am today.

First of all, I would like to give my sincere gratitude to my two supervisors Tatiana Filatova and Ariana Need. These two women have been an incredible inspiration for me, and they supported me in every aspect of $\mathrm{my} \mathrm{PhD}$ and professional lives. Tatiana, you shared your knowledge, wisdom and experience with me. You helped me to structure my thoughts and you motivated me to get the best out of myself. I do not believe that anyone has ever managed to motivate me in my work in the way that you did. You made me explore my full capacity, which made me grow tremendously over the last four years. Thank you for that. I also really appreciate that you were there for me on a personal level. I remember our meetings that turned into walks around the campus, during which you gave me the mental support I needed at the time. Ariana, you were like a mentor to me. You were always so kind and enthusiastic. I really admire your warm personality. You always made me feel more optimistic after our meetings, since you always saw an exciting new opportunity in every set-back. You were always there for me when I had personal struggles during my work. You stimulated me to explore my potential and to make strategic decisions that will help me in my future career. Thank you for that.

I would like to thank Paul Bin for his incredible commitment in supporting me, even though he was not formally appointed as my supervisor. We met over skype at the beginning of my $\mathrm{PhD}$, and he has been there for me ever since. Paul, you worked so hard arranging everything for my stay at ECU, and arranging all the administrative stuff to get my survey running. I cannot overstate how grateful I am for the things that you have done for me. In North Carolina you received me so warmly, introducing me to your colleagues at the Economics Department and Natural Hazards Center, taking me out for dinner and showing me around in the fitness centre. I remember meeting your wife when we had dinner together at the Sanitary Fish Market and Restaurant in Morehead City. Thank you for making my stay in North Carolina so enjoyable. I also want to say that it has been a great pleasure writing papers together with you. I hope we can continue to be in touch in the future.

My gratitude extends to Edward Halteman from Survey Design and Analysis. He has helped me formulating the survey questions to address the target audience, putting the surveys online, and distributing the surveys to the target audience in my case study area. Without 
his help it would not have been possible to get the data I needed to write my last two papers.

Many thanks to everyone who has made my life in Twente, at the university as well as outside. My colleagues in the L.I.K.E. room, as well as other colleagues from CSTM, with whom I enjoyed many many coffee breaks (bedankt Mariet dat jij iedere keer weer de perfecte koffie voor me klaarmaakte), lunch breaks and long lunch walks. Sorry to those who I dragged along for a lunch walk that was slightly too long. Thank you to the members of the PhD Initiative Group and the P-NUT board whose company I enjoyed in work that was a nice distraction from the normal $\mathrm{PhD}$ and researcher life. You are all very nice people, and I am happy to have had the opportunity to meet you and work with you during my $\mathrm{PhD}$. Thank you to all of those who have helped me skate ever faster laps on the $\mathbf{4 0 0}$ metre track (42.34 sec. on the 500 metre). Speed skating has always been a great way for me to blow off steam, and I would like to thank everyone from DSV de Skeuvel who has shared this with me. Bedankt dat jullie me uit de wind hielden op de lange intervallen waar ik helemaal stuk ging, en bedankt trainers voor de technische aanwijzingen die ik nodig had om harder te gaan schaatsen.

Thank you to my dear friends who have always supported me and believed in me. Cors, Ali, bedankt dat jullie aan mijn zijde staan tijdens de verdediging van mijn proefschrift, en bedankt dat jullie er altijd voor me waren. Maartje, bedankt dat je altijd in mij geloofde en bedankt dat jij mij steunde op de momenten dat ik worstelde met mijn PhD. Ik weet dat ik niet makkelijk ben geweest op die momenten. Anna, Ewert, Djaner, Festus, Neiske, Mandy, Babeth, Lisanne, Sjeng, Bas, Jos, Misghina, bedankt voor jullie vriendschap. Ik weet dat ik altijd op jullie kan bouwen. Bedankt voor het delen van alle mooie momenten, borrels, diners, lekkere biertjes, lange fietstochten, wandelingen, uitjes, voetbalwedstrijden en gezelligheid. Dit heeft mij altijd goed gedaan.

Als laatste wil ik graag mijn familie bedanken voor hun onvoorwaardelijke steun en liefde. Pap, mam, zonder jullie was ik nooit zo ver gekomen. Jullie hebben mij alles gegeven wat ik nodig had om dit te bereiken. Lieve Anouk en Tim, ik ben trots op jullie, en dat gebruik ik graag als inspiratie voor de dingen die ik zelf in het leven wil bereiken. Ik hou van jullie. Lieve Franzi, mijn grote liefde, mijn maatje, mijn steun en toeverlaat, dankjewel dat jij aan mijn zijde staat. 


\section{CONTENTS}

CHAPTER I

Introduction

CHAPTER 2

Improved Methods for Predicting Property Prices in

29

Hazard-prone Dynamic Markets

CHAPTER 3

Bridging the Gap Between Revealed and Stated

Preferences in Flood-prone Housing Markets

CHAPTER 4

Capitalization of Flood Insurance and Risk

73

Perceptions in Housing Prices: An Empirical Agent-

Based Model Approach

CHAPTER 5

Evading or Mitigating Flooding: Bottom-up Drivers

99

of Urban Resilience to Climate Change

CHAPTER 6

Repetitive Floods Intensify Outmigration and

Climate Gentrification in Coastal Cities

CHAPTER 7

Discussion and synthesis

Bibliography

Appendixes

Supplementary List of abbreviations, List of figures, List of tables

Summary

Samenvatting 

CHAPTER I - Introduction

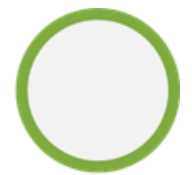

00 



\section{I.I Grand scientific challenges}

We currently live in an era where the human population and technology have an enormous impact on the environment. It has reached an extent that scientists consider that we have entered a new geological epoch: the Anthropocene (Crutzen 2002). Our global population, wealth and technology are still increasing, and so is our role in shaping our planet. This gives huge responsibilities to modern humans, in particular to scientists, engineers and policy makers. Climate change (Stern 2006; Stern 2008), land degradation (Admundson et al. 20I5; FAO, ITPS 2015) and rapid decline of global biodiversity (Leadley 2010; IPBES 2018) are among the major collective impacts of human action on our planet. Without putting a value judgement on these trends, it is obvious that some trends happen faster than we anticipate, some impacts are accelerating beyond our control (Steffen et al. 2018), and some developments are contradicting our interests, causing conflict and war over resources (Board 2007; Reuveny 2007). Therefore, societies need to adapt and scientists have a leading role in figuring out how. We need to understand how we shape the future of our planet, what the consequences of our collective actions are on a large scale, and what we can do to prevent undesired changes on our home planet (Bai et al. 2016).

The acknowledgement of these facts gives rise to the paradigm that societies and natural systems are not independent entities. The impacts of humans on the environment and the human-environment/environment-human interactions are often very complex. The role for scientists starts with understanding how people interact with the environment and vice versa. It introduces a new discipline of environmental science acknowledging that environmental systems and human systems are inextricably linked. They are interrelated and should be treated as such when addressing global environmental change issues. In 1993 Paul C. Stern called for connecting social science disciplines with environmental sciences as a holistic approach in addressing the scientific challenges of today (Stern 1993). Now, 25 years later, this paradigm has been extensively adopted in environmental science (Liu et al. 2007, Milner-Gulland 2012, An and Lopéz-Car 2012). Studies of natural systems increasingly include humans as part of integrated systems, and vice versa - social sciences fields increasingly include environmental system dynamics in their assessments (Nordhaus, 2018). These integrated systems are referred to as Coupled Human And Natural Systems (CHANS). Alternative names in the literature are: coupled natural and human $(\mathrm{CNH})$ systems, coupled human-environment systems (CHES), and socio-environmental or social-ecological systems (SES). I will mostly 
address them as CHANS, although I may use the terms interchangeably throughout this thesis.

\section{I.2 Modelling of complex systems}

Complexity is an inevitable feature that characterises CHANS. Due to the many elements and interactions it is not always easy to trace the cause-effect relationships in CHANS. Ecosystems contain many different species interacting on different trophic levels, and environmental conditions influence ecosystems' functioning and vice versa. They may undergo regime shifts and trophic cascades (Carpenter and Kitchell 1996; Scheffer et al. 2005). On top of that we have complexity in human systems: policies, laws and institutions acting on different levels of society, stakeholders operating with a diversity of interests, and communities having various social and cultural norms that influence people's behaviour. Like ecosystems, human systems are also subject to critical transitions. For example people may switch to migration when climate change causes catastrophic floods, droughts and food insecurity (Reuveny 2007; Penning-Rowsell 20II; Faist and Schade 20I3). The interactions in CHANS are characterised by nonlinearities, feedbacks, thresholds, surprises, heterogeneity and time lags (Liu et al. 2007), which make systems' dynamics particularly hard to predict. Particularly when environmental conditions change into the unexplored realm, where we are challenged to extrapolate the known into the unknown. Thresholds can be exceeded unexpectedly, causing sudden catastrophic changes in CHANS' appearance and functioning. Surprises happen when we overlook key components and processes in CHANS, in which case impacts can occur that we have not foreseen.

It is for these reasons that we rely on computational simulation models, which help us better understand CHANS in terms of how they function, how the elements interact with each other, what the dynamics are and how the systems change over time (Verburg et al. 2016). Computational simulation models can help us understand how humans interact with the natural system and vice versa. The complexity of CHANS make it difficult to grasp intuitively what the cause-effect relationships are, and therefore we can use modelling as an approach to disentangle the black box (Matthews et al. 2007). If done right, our models can be used to explore future scenarios and policy options that may help preventing (mitigation) or responding to (adaptation) undesired impacts of human actions on our natural systems and our well-being (Magliocca et al. 2013). Model simulations can be seen as a virtual lab for scientists and policy makers, as they provide a safe virtual environment to explore 
assumptions about the system and the consequences of various policy strategies (Gilber and Terna 2000; An 2012). Simulation models allow us to run large scale experiments in CHANS to trace human-driven global- and regional change that would otherwise take years to monitor (Railsback and Grimm 20I I).

Hence, models are an essential tool in addressing anthropogenic climate change, land degradation and rapid loss of biodiversity. There are numerous modelling approaches that can be (and have been) adopted for this purpose. Yet, there are a number of essential requirements that are worth mentioning. These requirements are broad and inspired by Verburg et al. (2016) and by Filatova et al. (2016). In order to make our models of CHANS useful, they should at least be capable of:

I. Capturing feedbacks between human and natural systems. The impact of human activities on natural systems and vice versa must explicitly be modelled. There is a wide range of possibilities of how this is done, and not all models contain two-way feedbacks (human-nature, nature-human). Ideally a model should capture a range of feedbacks simultaneously and form closed loops in order to capture the system as a whole, because the complexity of CHANS arises when many feedbacks operate at the same time.

2. Accounting for regime shifts. Regime shifts are radical and structural changes in the functions or processes of a system, as opposed to gradual changes in the state of a system following a trend in time (Folke 2006; Filatova et al. 2016). Although not all CHANS undergo regime shifts, the potential for regime shifts is increasing in many CHANS as we risk reaching a planetary threshold in the Anthropocene (Steffen et al. 2018). Regime shifts can emerge as a result of various drivers: they can be driven by internal change as well as by external pressure (although the definition of internal and external also depends on how the boundaries of the system are defined), and they can be caused by gradual change (e.g. gradual increase in global temperatures and sea levels) or by sudden shocks (e.g. heatwaves, droughts and floods). Other related concepts in various fields are: 'non-marginal changes', 'systemic shocks', 'tipping points' and 'critical transitions'. I use these terms interchangeably throughout my thesis.

It should be noted that complex systems are highly dynamic and that they are constantly subject to change, which makes it hard to strictly define regimes and equilibria for CHANS, and so is distinguishing a marginal from a non-marginal change in a system. Structural changes in systems may manifest themselves only in parts of the system, while the system as a whole is still identified as the same entity. Think for example about gentrification in certain neighbourhoods of a city due to improved 
environmental conditions. While neighbourhoods undergo structural demographic changes in this process, the function and identification of the city as a whole remains the same. Therefore one can argue whether capturing identifiable regime shifts is an appropriate requirement from models. At least a model must operate well under conditions out-of-equilibrium, and account for adaptive behaviour and learning of human actors - let's call them non-static conditions. They must at least capture systemic behaviour that undergoes changes of any nature and velocity. A model should operate well when we relax the assumptions of identifiable stable equilibria.

3. Working well for prediction and exploration purposes. Models are often developed for prediction purposes, in addition to serving other purposes including co-learning or understanding systems rather than forecasting. The accuracy of the predictions depends on a variety of aspects such as the complexity of the system and the model's ability to deal with these complexity aspects. In the case of CHANS in the Anthropocene, especially those that are subject to unprecedented changes in functioning, it is important that a model is flexible enough to capture system behaviour in the unexplored realm. This demands models that include all previous points: capturing the feedbacks between human and natural systems, being able to capture out-of-equilibrium dynamics shifts and capturing the complexity aspects. Moreover, models need to be properly validated and calibrated, and behave well when the predictions are extrapolated - e.g. when environmental conditions in the future change beyond historical conditions on which the models are calibrated. This complicates the search for proper validation sources, because we are not at that stage yet. For example: how coastal cities adapt when sea levels rise by one metre is hard to explore in our current situation because the sea has not risen by that much yet and waiting for that to happen is simply too risky. Lastly, models should be suitable for providing a virtual laboratory in which the impacts of mitigation and adaptation policies can be explored. Apart from simply exploring how CHANS evolve in the future, it is important that models can also show how undesired pathways can be avoided, and explore which policy actions could be effective in doing so.

\section{I.3 Agent-based modelling as a tool to model CHANS}

There are numerous approaches to consider when capturing CHANS in models. A few examples are: Bayesian networks, conceptual mapping, fuzzy cognitive maps, causal loop diagrams, agent-based modelling, regression models, structural equation modelling, system dynamic modelling, and computable general equilibrium models. 
Gotts et al. (2018) categorise these into four general approaches: conceptual, statistical, mathematical and simulation modelling. Various reviews exist on the various modelling approaches applied to CHANS (Kelly et al. 2013, Filatova et al. 2016, Gotts et al. 2018) that may classify the approaches in different ways. Modellers often do not use a single distinctive modelling approach, and many models of hybrid approaches exist. Reviewing all of these is beyond the scope of my thesis. I would like to present one model in particular as a promising approach to addressing the global challenges of the Anthropocene: agent-based modelling (henceforth: $A B M$ ) (Farmer et al. 2015; Stern 2016). I have chosen ABM mainly because it has the potential to cover all of the aforementioned modelling requirements.

In $A B M$, systems are modelled through sets of behavioural rules that operate at the micro level. The rationale behind the method is that complex systemic behaviour emerges from the bottom up through autonomous behaviour and interaction of many individual decision makers (agents) in the system. ABM is particularly powerful in capturing all aspects of complexity of CHANS. Heterogeneity, non-linearity, individual and social learning, interactions, adaptive behaviour, and emergence are inherently connected with ABM (Farmer and Foley 2009; An 2012; Tesfatsion 2002; Matthews et al. 2007; Gilbert and Terna 2000; Bonabeau 2002). By taking into account heterogeneity, bounded rationality and learning, $A B M s$ allow for a realistic representation of human behaviour. Agents are in constant interaction with their environment, which makes $A B M$ particularly useful in capturing feedbacks between human behaviour and the natural system and vice versa. Furthermore, ABM works exceptionally well for studying out-of-equilibrium dynamics. It allows us to relax the assumption of stable equilibria and to capture the dynamics of complex systems that are constantly subject to change. In $A B M$ we can study the emergence of nonmarginal changes in systemic functioning that are driven by bottom-up processes (Gilbert and Terna 2000; Farmer and Foley 2009; Railsback and Grimm 20I I; An 20I2). It allows us to trace back the bottom-up emergence of all sorts of regime shifts that are either caused by gradual changes or by systemic shocks, both internally and externally.

$A B M s$ are highly complex and although they are readily widely adopted in various applications, the field of $A B M$ is still far from mature. ABMs are typically less suitable for prediction purposes and more for understanding system dynamics (Kelly et al. 20I3), and consequently ABMs have not been used a lot for prediction purposes nor for solving real-world policy problems (Matthews et al. 2007; Verburg et al. 2016; Schulze et al. 2017). There is a need to invest further in ABM to make the method more suitable for the purpose of prediction and as a decision making tool. This entails 
a few key methodological issues in $A B M$ that need particular attention. Most importantly, we need to focus on the way human behaviour is formalised in mathematical models (Gotts et al. 2018), take into account adaptive behaviour and learning, and focus on validation and verification of behavioural rules of human agents in our model. Before I proceed with the research gap and research questions, I will briefly review a few ways in how human behaviour is typically addressed in ABM of CHANS.

\section{II.4 Human behaviour in ABM: the status quo}

\section{I.4.I Rational decision making}

Utility maximisation is the simplest and most straightforward theoretical approach to modelling human behaviour. It is therefore the theory most commonly applied in ABMs of Land Use/Cover Change that are not informed by empirical data (Groeneveld et al. 2017). This is complemented by the expected utility theory of Von Neumann and Morgenstern (1953) in the context of decisions under risk, for example in the case of climate-driven natural hazards. Expected utility theory is based on the assumption that people weigh all options by their expected gains and losses, and choose the option that gives the highest utility within their budget constraint. It is assumed that people are fully informed, self-interested, optimising rational agents and that they perceive all information objectively. The drawbacks of this homo economicus model has long been debated (Simon 1956; Gigerenzer and Selten 2002; Groeneveld et al. 2017), mainly because the assumptions are oversimplified. Social behaviour, habits and norms are ignored, people can never have full access to all information, and the theory fails to account for suboptimal behaviour (e.g. when satisficing instead of optimising) (Gigerenzer and Goldstein 1996).

\subsubsection{Bounded rationality and choice of a theory}

Alternatively, to go beyond the oversimplified rationality and optimisation assumptions, one can include principles of bounded rationality in agent behaviour (Simon 1997). I do not intend to enter the discussion of what behaviour can be considered rational and what not, as the interpretations differ per scientific field (Mueller and De Haan 2009). In this section I will discuss alternative behavioural theories used in ABM that deviates from the above-mentioned perfectly rational agent that is fully self-interested, has full information and always chooses the best option that is available to her. Bounded rational behaviour can be included in a model either by adding it to the utility maximisation functions or by choosing alternative theories. Despite the simplified assumptions of utility maximisation it is possible to 
adopt the principles of bounded rationality in expected utility equations (Brown and Robinson 2006). However, the variations of attempts are large and they seem more like a mathematical trick than theoretically or empirically grounded solutions. Bounded rationality is not grounded in expected utility theory, and deviations from perfect rationality therefore require empirical data to validate and parametrise. An alternative is to adopt more complex models that use theories from behavioural sciences and artificial intelligence (Filatova 2016). Examples are prospect theory (Mueller and De Haan 2009), the theory of planned behaviour (Richetin et al. 2010; Rai and Robinson 2015), the model of goal directed behaviour (Richetin et al. 2010), protection motivation theory (van Duinen 2015) and the social amplification of risk framework (Van Duinen et al. 2016).

The inclusion of bounded rational agents that deviate from perfectly rational agents is driven by the desire to model human behaviour more realistically. As such, it is important to focus on what we can learn from other disciplines in social sciences beyond the economic rational agent (Groeneveld et al. 2017; Filatova et al. 2016; Müller-Hansen et al. 2017, Schlüter et al. 2017). Yet, alternative approaches and theories that capture bounded rational behaviour also need more data to parametrise than expected utility theory and they are less suitable for prediction purposes. Utility maximisation is a good model to start with when empirical data on micro-level behaviour is limited, and more complex theories of human behaviour can only be implemented once more data is available for validation and parameterisation of the theoretical assumptions. Another problem with many behavioural theories from psychology and sociology is that they often cover only part of the cognitive processes that drive behaviour. For example: the social amplification of risk framework describes how social networks, opinion leaders and (social) media drive risk perceptions, but additional theory is needed to describe how risk perceptions affect decision making. Finally, a lot of behavioural theories from psychology and sociology are not formulated in a way that they can directly be implemented in a model. They describe general concepts that are relevant in driving human behaviour (e.g. social networks, habits, perceived risk, fear, social norms), but the operationalisation of these concepts are not as clearly described in terms of quantifiable variables (like utility in expected utility theory). As such, the modeller has many degrees of freedom as to which theories to use or to combine, how to operationalise the concepts and how to model the decision rules (maximisation, satisficing, random) (Muelder and Filatova 2018). 


\section{I.4.3 Empirical data}

As mentioned before, a lot of behavioural theories require empirical data for parameterisation of the behavioural variables that are adopted in the model. One can also choose to omit behavioural theories completely and fully rely on empirical data. The majority of ABMs on land use/cover change use empirical data to inform decision rules without the explicit use of any theory (Groeneveld et al. 2017). The advantage of this approach is that a modeller does not have to choose between a large variety of behavioural models, and she is not forced to define parameters for all the behavioural variables that comprise the chosen theory. Instead, the empirical data can be used to find statistical correlations between behavioural, cognitive or even demographic variables and the behavioural outcome (Valbuena et al. 2008, Smajgl and Bohensky 2013). Moreover - provided that the right data is used - empirical data gives a detailed insight in actual behavioural patterns on the micro scale. It supports the strength of ABM by allowing for parameterisation of heterogeneous agents, and it can be used to validate or verify the behavioural and theoretical assumptions in the model.

There are a few concerns to consider when relying on empirical data. Firstly, the problem with $\mathrm{ABM}$ s that are solely informed by empirical data is that the behavioural rules are often ad-hoc, and therefore difficult to generalise (Janssen and Ostrom 2006; Smajgl et al. 20 I I). Secondly, the level of detail and the amount of data needed make it difficult to extrapolate the behaviour in time. Surveys, interviews and lab or field experiments are often done at a single point in time, although modern information technologies such as social media and smart-phones may enhance the frequency of interactions (Bell 2017). The lack of longitudinal data makes it hard to identify any trends in the empirically observed behaviour (Janssen and Ostrom 2006). Thirdly, data collection is very costly and time consuming, and the question is whether the use of data is better than using well-established theories and common sense. This is also a matter of a costs and benefits, and the resources at hand. Fourthly, as data collection is often very time consuming and costly, it is important to make the right decisions on how data is collected and with what purpose.

In the next part I will discuss two important research gaps that still remain in the modelling of human behaviour in ABMs of CHANS. 


\section{I.5 Research gaps}

There are many degrees of freedom in terms of how to model human behaviour in ABMs of CHANS, and guidance is necessary to make strategic modelling decisions

The overview of the status quo sheds light on the variety of ways how human behaviour is modelled in ABM. There are many degrees of freedom of how human behaviour is captured in our models (Gotts et al. 2018). Fig. I.I presents three steps where modellers are faced with a choice. First, at the conceptualisation of human behaviour a trade-off needs to be made on the level of complexity by which the model will represent human behaviour (Sun et al. 2016). Secondly, modellers need to choose among many alternative ways to validate human behaviour in ABMs of CHANS. Thirdly, at the operationalisation there are still many degrees of freedom on how data can be used to parametrise the behavioural model, and how the theories are implemented in the model in terms of mathematical equations.

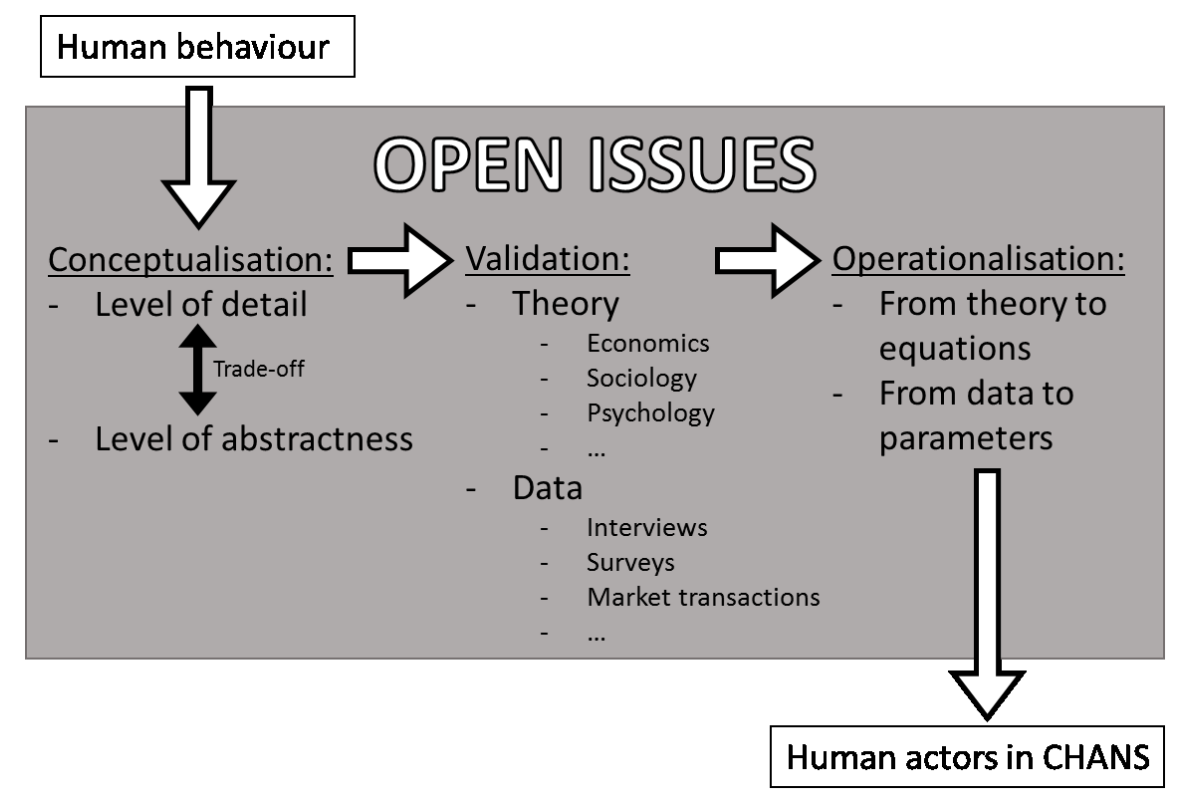

Figure I.I. Open challenges for modellers how to capture human behaviour in ABM. The three steps 'Conceptualisation', 'Validation', and 'Operationalisation' highlight where the modeller is faced with a choice. The arrows represent a typical pathway of the choices that modellers go through, although they may not necessarily follow this particular order, and dependencies between the three steps do exist. 
The fact that every agent-based modeller has to go through these challenges calls for proper guidance in order to follow good modelling practices. Some guidance is provided in a review by An (20I2), discussing the pros and cons of various ways of informing behavioural rules for agents in a model. Another important step is taken by Schlüter et al. (2017) who developed a framework to assist agent-based modellers of CHANS in structuring the formalisation of human behaviour in their models. It is an addition to the ODD+D protocol (Müller et al. 2013) that facilitates structuring the design of $A B M s$ and communicating them among modellers. The work of Schlüter et al. (2017) focuses on how modellers can incorporate theories of human decision making in models of CHANS. Their framework facilitates in mapping existing behavioural theories in such a way that they can be incorporated in an agent-based model. These are good developments, but still their framework does not provide assistance in how to make the trade-off between detail and abstractness, how to select the behavioural theories, and what to do with empirical data.

\section{Dynamic human behaviour is underrepresented in ABMs of CHANS}

Another major issue is the static representation of human behaviour, which is a common property of many ABMs of CHANS. The framework of Schlüter et al. (2017) is also rather static. As they themselves point out: "Our framework does not neatly capture processes that go beyond a single time step, such as learning." Humans are highly adaptive in their behaviour. Learning and change in behaviour becomes particularly relevant when global environmental changes alter context of the decision making. Their decisions will change as they learn to adapt to changes in the environment around them, which is not adequately taken into account in our models. Given the severe impacts of collective human action on natural systems it is essential that we capture the dynamic behavioural aspects of humans in CHANS adequately (Stern 1993; Milner-Gulland 2012; An 2012, Stern 2016). The effectiveness of policies aimed at sustainable use of natural resources is at stake when adaptive behaviour is not adequately represented in our models of CHANS. Policies designed to mitigate negative impacts of humans on the environment are ineffective when they are based on the wrong assumptions. This demands a deeper understanding of the behavioural drivers behind the choices that people make, and how these change over time as people interact with each other and the environment (Stern 1993; Milner-Gulland 2012). 


\section{I.6 Research goal, research questions and thesis}

\section{structure}

In the light of improving the prediction value of our ABMs of CHANS, I will focus on the operationalisation of dynamic human behaviour in models. We are capable of generating forecasts with complex geophysical models of the environment, emphasising the dynamics of environmental processes in a changing world. Yet, the complex dynamics of human behaviour and its impact on the environment is still underrepresented in CHANS prediction models (Stern 2016). The methodological focus of this thesis contributes to addressing the grand scientific challenges that humanity faces in the Anthropocene, such as climate change, land degradation and biodiversity loss. This leads to the following research goal:

Improving the modelling of human behaviour in coupled human and natural systems, in agent-based models that are designed for exploring global change scenarios and policy options

This goal encompasses our responsibility as modellers to go beyond understanding systems in the present. We must try to understand and predict how the world will change as a consequence of human action. I use the word 'exploring' because prediction suggests a precision that we will probably not achieve in complex systems like CHANS. 'Exploring' refers to the assessment of various assumptions on the micro level, and how they might influence the patterns on the macro level. It is not about being precisely right with our predictions, but about understanding the patterns that may emerge in societies and natural systems in the future as a consequence of the expanding collective impact of human behaviour. Furthermore, I address the potential for these models to serve as a virtual laboratory to support policy decisions. Apart from assessing what may happen, how societies and natural systems may change in the future, these models must be capable of assessing how certain policy strategies affect people's behaviour, in an attempt to mitigate the undesired consequences of our behaviour. In my thesis I address some key methodological issues that may help ABM move forward in serving these purposes.

I address the research goal by means of a case study of flood-prone housing markets in the U.S. An ABM has been designed to capture dynamics of a market where flood risk drives people's choices where to live and how much to pay/ask for their home. The case study addresses the modelling of human behaviour in case of risk and uncertainty. I show step-by-step improvements to the existing ABM of flood-prone housing markets as an illustrative example of how to tackle human behaviour in ABMs 
of CHANS. These improvements are achieved in response to the research questions addressed in the following chapters:

RQ I: How can the collective preferences of a variety of individuals be captured in a dynamic environment? (Chapter 2)

$A B M$ is focused on modelling the behaviour of individual agents, and thus it is important to trace the dynamics of goals, preferences, perceptions, states and decisions of all individual agents in the model. Yet, the system dynamics are equally important, particularly when there are many feedbacks in the system between the autonomous agents and their environment. Agents constantly perceive their environment, act according to the environmental conditions and their goals, and they learn from their behaviour and adapt their strategies accordingly. For modellers this requires giving the agents something to perceive. The environment in an $A B M$ is highly abstract, and consists of lots of dynamic variables that can be measured and tracked during the simulations. It is our job to identify which variables are relevant during the simulations to track in order to provide an accurate representation of the state of the environment that agents perceive and base their decisions on.

Furthermore, a first validation exercise for $A B M s$ is to adequately simulate the aggregate patterns that emerge from a collective of heterogeneous agents behaving and interacting. CHANS are vastly complex, and so are the outcomes of agent-based models that try to describe them. It is not sensible to validate and verify all the microlevel outcomes of the model, because ABMs are not designed for predicting the exact choices and outcomes for each individual agent. They are instrumental in modelling complex systemic behaviour by focusing on bottom-up dynamics, and therefore we should focus on the aggregate patterns that emerge in ABMs rather than tracking individuals (Grimm et al. 2005). In order to do so we must be able to capture the aggregate patterns well in the first place.

RQ2: What are the aggregate impacts of various behavioural theories and assumptions regarding risky choices? (Chapter 3 and 4)

Chapters 3 and 4 are the first chapters in which I run simulations of a flood risk ABM. As a first exercise of addressing the knowledge gap - I need to determine if should I use theory, data or both, and which theory to choose - I will start with a mostly theory based $A B M$ in these chapters. Theories are the easiest to start with because 
they do not necessarily require empirical data to implement them in the model. There are many behavioural theories to choose from, but I start with the ones that are more on the side of rational decision making and simple forms of bounded rationality. The advantage of these models/theories is that they require little to no micro level empirical data to develop parameters, and hence it is fairly simple to implement them in the ABM. In chapter $3 \mathrm{I}$ address which micro-level behavioural theory of risk perception matches closest with the price patterns (of risk-prone and safe properties) observed in a set of historical transaction data. At the core of my thesis is the modelling of macro-level patterns that emerge in CHANS through micro-level behaviour of many heterogeneous interacting agents. In that regard chapter 3 also serves as a first sensitivity analysis: how the choice of a behavioural theory impacts the simulated outcome on macro scale, and thus how sensitive the model output is to the input assumptions of micro-level behaviour. The simulated transactions are validated on aggregate patterns in historical transactions, by using the metrics of chapter 2 .

Chapter 3 also brings about an operationalisation issue: some behavioural theories are not sufficiently elaborate to be implemented directly in an ABM. For many theories, especially those with many variables and those that are abstractly formulated, there are many degrees of freedom in how to operationalise some basic elements of the theory in an ABM. In chapter 3 I therefore run multiple possible operationalisations of the same theory (prospect theory) in the ABM.

Chapter 4 serves as the first policy experiment of the ABM. In this case study I test the impact of an insurance-enforcement policy under various behavioural assumptions. This chapter serves as a second sensitivity analysis: I assess the effectiveness of a certain policy strategy in achieving its intended goal, and meanwhile I assess how sensitive the implications of the results are to the micro-level behavioural assumptions/theories I use as input.

RQ 3: How well do various behavioural theories fit with empirical micro level data of household behaviour in various contexts? (Chapter 5)

Building on Chapters 3 and 4, in Chapter 5 I use empirical data to validate a number of behavioural theories of risk and uncertainty. The use of empirical data allows for the inclusion of more complex theories of bounded rational decision making in ABMs. In chapter 5 I further address the knowledge gap on whether to use theory, data or both in more detail. I hold interviews among real estate agents and I run 
questionnaires among buyers and sellers in other to better understand the microlevel behavioural choices that drive market dynamics. The use of empirical microlevel data at this stage is driven by the desire to understand the context-specific decision making in more detail. Naturally, an increased level of detail leads to more realistic representation of human behaviour in ABM. Yet, a more realistic representation of human behaviour may also result in redundant complexity of our models. Hence, the knowledge gap does not simply require an answer whether theory is better than data or vice versa, but it also deals with the cost-effectiveness (in terms of time and money) of adding more data and complexity to our models.

Furthermore, chapter 5 deals with the context of decision making. Some behavioural theories may be more adequate than others in capturing and predicting human decisions, which depends also on the context of the decisions. The use of empirical data is a good way to validate an $A B M$ and verify which theories adequately cover the important drivers of human decision making in which context. I address this issue in the case study by looking at various decisions of households (whether or not to self-protect against flooding, to avoid buying properties the flood zone, abandoning the hazard zone) throughout the flood-prone coastal U.S. I cover areas that have recently been flooded as well as in areas that have not been flooded for a long time. Furthermore, I include both buyers and sellers, households that live inside high-risk flood zones, households that live in safe areas and households that have experienced a flood. This data should cover the dynamics of how people with different roles in the market respond before and immediately after a major flood in their town, and what happens when people's houses get flooded, which drastically alters their information and perceptions. The 'context' in this example refers to the kind of decisions that people must make as buyers or sellers, the information that they obtain from flood events and how they perceive this information.

RQ4: Can this $A B M$ be used to simulate the emergence of regime shifts from the bottom up, when changes in the natural system affect changes in human behaviour? (Chapter 6)

Any model can be used for prediction purposes, but how well your model performs in predicting the future - in particular a highly uncertain one - remains an open question. The validity of a model's predictions is also judged by our confidence in the design and basic assumptions of the model - how well are these validated, and can we assure that the model captures a plausible future? Where chapters 2-5 are focused on improving the formalisation of human behaviour in ABMs of CHANS and 
gaining confidence in a model's performance, chapter 6 serves as an experiment where the model is put to the test.

In chapter $6 \mathrm{I}$ assess the model's potential to capture the future in a globally changing environment. Non-marginal transitions or regime shifts are one of the major consequences of global change in many CHANS. In my thesis I use ABM as an instrument partly because of its capabilities to capture such systemic changes. In ABM we can simulate the emergence of systemic transitions from the bottom up, using our understanding of how individuals respond to changes in the environment. Regime shifts can be governed by internal as well as external drivers, due to gradual forcing (e.g. gradual temperature increase and sea level rise) as well as by shocks (e.g. floods, fires, diseases) (Scheffer 2009). In the case study of my thesis I simulate the regime shifts that are caused by sudden shocks, namely floods. I use micro level empirical data that represents an example of how people respond to severe floods. I combine empirical data with theory to form a mechanistic understanding of what happens when severe floods become more frequent and widespread in the future. And finally I scale up micro level behavioural patterns in an agent-based simulation experiment to predict how an increase in severe floods may drive shifts in macro level patterns such as demographic changes in coastal urban areas.

Moreover, chapter 6 shows the complexity of the output of an ABM and the benefits thereof. I will discuss the multidimensionality of the model's output: an infinite number of output parameters that can be analysed, of which the dynamics are often equally diverse. Regime shifts may manifest themselves in some facets of the system, while other aspects that characterise the system remain stable. In chapter 6 I show a variety of patterns that emerge in a number of macro level variables that are relevant indicators of the system's functioning and outlook. The diversity of these patterns and the diversity of conclusions that are drawn if they are assessed separately indicates why ABM does justice to the complexity of CHANS more than any other modelling technique.

\section{I.7 Case study background}

I use a case study of flood risk in climate-sensitive urban areas as an illustrative example of how to capture human behaviour in ABMs of CHANS. In this particular case study I am faced with the challenge of modelling how households in the housing market respond to flood risk, and how this affects the market dynamics and demographics in coastal urban areas. I work with an ABM that is designed to assess and predict the economic and social consequences of climate change in flood-prone 
property markets (Filatova 20I5). The modelling of human behaviour is thus primarily focused on a situation of risk and uncertainty, for which many theories exist in various social sciences fields. I test this model by changing the human behaviour component of the model significantly, in line with the goal and research questions of this thesis. In this chapter I will briefly discuss some background of this case study. Economic damage by floods has globally increased over the last decades as a result of increased frequency and severity of flood events, and due to increased property capital in coastal areas and river floodplains (Kunreuther and Michel-Kerjan 2007, Kousky 2010, Atreya and Ferreira 2012). Yet, the risk of flooding is often not well capitalised into property values (Atreya and Ferreira 20I2, Bin and Landry 20I3), mostly due to incomplete information of the actors in property markets (Chivers and Flores 2002, Burningham et al. 2008). People are willing to pay high prices for properties that are associated with environmental amenities such as proximity to the beach or adjacency to water, despite that these amenities are often spatially correlated with risks (Donovan et al. 2007, Bin et al. 2008). Consequently, flood events can cause sudden price drops because they serve as a reminder of the potential hazards, which alters people's perception of the hazard. Yet, hedonic studies of property prices in flood-prone areas have shown that prices will slowly recover as people forget about the flood (Atreya and Ferreira 2012, Bin and Landry 20I3). These dynamics are illustrated in Fig. I.2, showing the sudden price drop after a flood event followed by recovery of the price towards the zero risk threshold. In

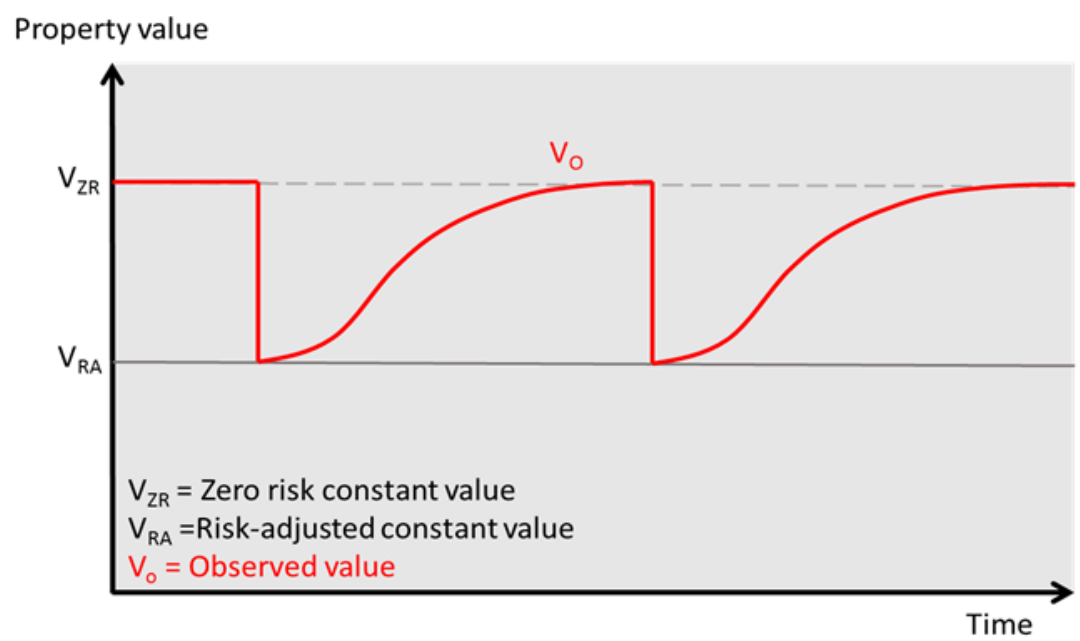

Figure 1.2. Projected property price with flood events whereby flood probability does not change over time. Note the sudden drop after the event and the slow recovery of the price. After Pryce et al. (2011) 
a market where people are fully informed and perceive risk objectively at all times, the property prices would be equal to the risk-adjusted price. In this case there would be no sudden drop and recovery of the property prices after a flood event. Pryce et al. (201 I) show that property markets that are not well adapted to natural hazards are prone to sudden and dramatic changes when the risk increases, illustrated in Fig. I.3. Initially the price recovers towards the zero-risk threshold, but as floods become more frequent this price will drop more strongly without recovering towards the zero-risk threshold. This scenario could be triggered by climate change, as the frequency and severity of natural hazards will increase (Stern 2006). Many urban areas will become more prone to flooding when global temperatures are increasing, mainly those that are flooded by severe hurricanes (Webster et al. 2005). The sudden shifts in property prices without recovery can have devastating consequences for the people that live there, as they will find themselves unable to adapt to these sudden changes. This poses a challenge for policy makers to come up with strategies to mitigate the potentially devastating impacts of climate change in flood-prone property markets and to design flood-risk management policies that can accommodate these changes in the coastal housing market regimes in a climate-changed world.

\section{Property value}

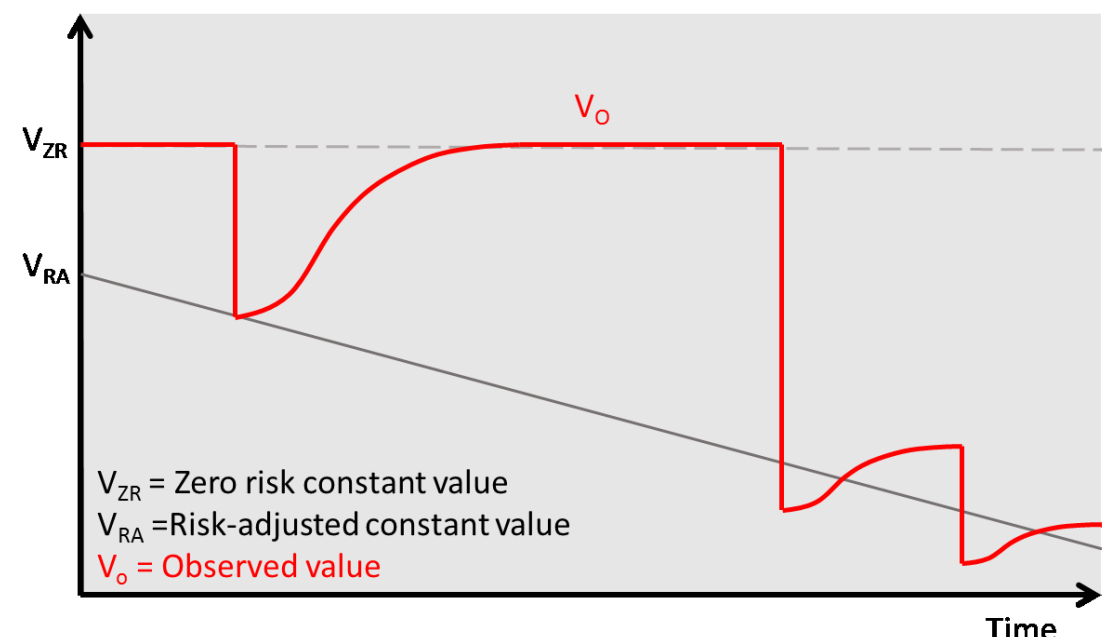

Figure 1.3. Projected property values over time with increasing flood risk. Note the sudden dramatic price drop after the second flood event, after which the value does not recover. After Pryce et al. (20II) 
The conceptual model of Pryce et al. (20II) is still very stylised and one-dimensional, looking only at property values and discarding any structural or demographic changes to property markets in response to hazard events. The predicted price dynamics can be validated with empirical observations of housing values in areas that have been affected by floods in the past, such as observed by Atreya and Ferreira (2012), and Bin and Landry (20/3). Although they provide a plausible explanation of the behavioural drivers behind these price dynamics, the theoretical support for this conceptual model is rather limited. Consequently, the concept may not hold in case of structural changes to the system and its functioning. Furthermore, by focusing merely on property values, the model is of limited use when studying the social and economic consequences of climate change in flood-prone urban areas.

Hence, an ABM has been developed by Filatova (20I5) to get a better understanding of the processes that drive the price dynamics in climate-prone coastal housing markets, and to study the consequences of climate change and regime shifts in coastal urban areas. The model supports a more holistic approach in studying the dynamics of markets in natural hazard areas in a changing climate, so that more effective policies can be adopted to mitigate the impacts of climate change and floods. In this thesis I build upon the model of Filatova (2015) and tackle step-by-step the challenges of capturing human behaviour in situations of risk and uncertainty more realistically, so that the model becomes more reliable in exploring how risk-prone urban areas might change over time as a consequence of climate change. The model captures the behaviour of buyers, sellers and a real estate agent. Homeowners decide to put their property up for sale and become sellers in the market. The role of the real estate agent is informing sellers about the asking price of the property. Buyers also enter the market and search for a property according to their preferences for various housing attributes, location and the level of risk. Sellers enter negotiation with buyers and they may come to an agreement on the price, after which the transactions are recorded by the real estate agent, who updates her price expectation on the basis of current market conditions, i.e.: the preferences of buyers and sellers in the market. My goal in this thesis is improving the prediction value of the model by capturing well the current and future preferences and behavioural choices of both buyers and sellers (especially those related to flood risk), and to be able to extrapolate how these agents might respond to increasing risk levels and flooding events. 


\title{
CHAPTER 2 - Improved Methods for Predicting Property Prices in Hazard-prone Dynamic Markets
}

This chapter is published as a journal article:

de Koning, K., Filatova, T., \& Bin, O. (2018). Improved Methods for Predicting Property Prices in Hazard Prone Dynamic Markets. Environmental and resource economics, 69(2), 247-263.

\begin{abstract}
Property prices are affected by changing market conditions, incomes and preferences of people. Price trends in natural hazard zones may shift significantly and abruptly after a disaster signalling structural systemic changes in property markets. It challenges accurate market assessments of property prices and capital at risk after major disasters. A rigorous prediction of property prices in this case should ideally be done based only on the most recent sales, which are likely to form a rather small dataset. Hedonic analysis has been long used to understand how various factors contribute to the housing price formation. Yet, the robustness of its assessment is undermined when the analysis needs to be performed on relatively small samples. The purpose of this study is to suggest a model that can be widely applicable and quickly calibrated in a changing environment. We systematically study four statistical models: starting from a typical standard hedonic function and gradually changing its functional specification by reducing the hedonic analysis to some basic property characteristics and apply kriging to control for neighbourhood effects. Across different sample sizes we find that the latter performs consistently better in the outof-sample predictions than other traditional price prediction methods. We present the specific improvements to the traditional spatial hedonic model that enhance the model's prediction accuracy. The improved model can be used to monitor price changes in risk-prone areas, accounting for changes in flood risk and at the same time controlling for autonomous market responses to flood risk.
\end{abstract}
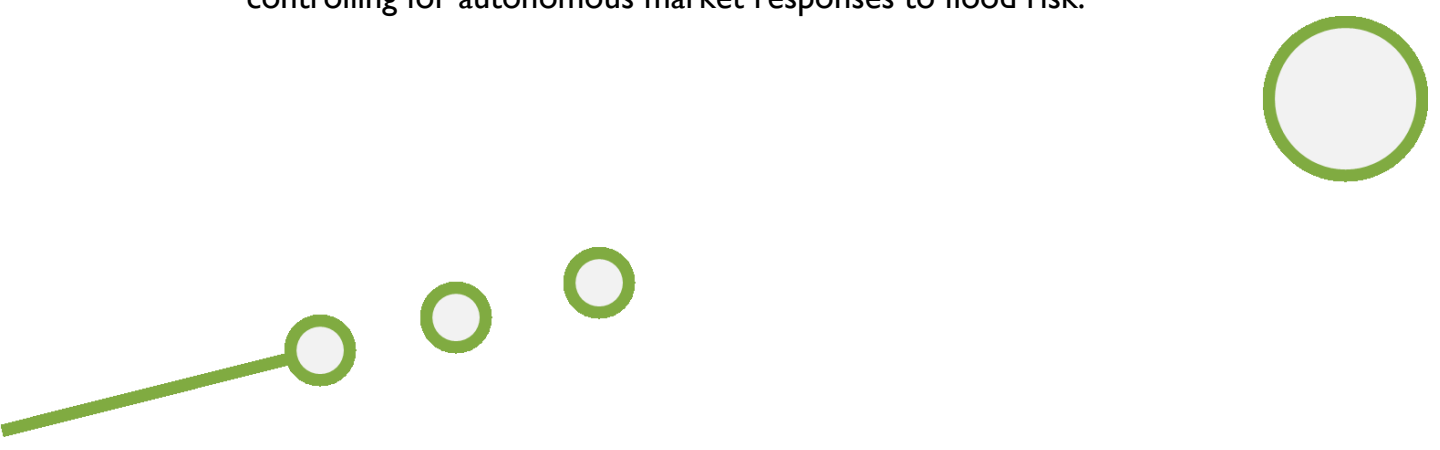



\section{2. || Introduction}

Housing contributes largely to the welfare of individuals. Consequently, housing prices can strongly influence households' financial decisions (Bostic et al. 2005) making households richer or poorer as prices fluctuate. Changes in property prices are driven by changes in macro-economic conditions, changes in consumer preferences and incomes, and exogenous shocks (Filatova 2014). At the times of natural disasters price tends to shift significantly and abruptly (Bin and Landry 2013, Atreya 2013) implying that there are systemic changes in property markets. In other words, transactions in the past may not be representative anymore when making current price assessments or projections for the future. Therefore it becomes important to utilize most recent sales in conducting reasonable market price assessments or predictions. Various comprehensive methods have been developed for these purposes in the past decades (Basu and Thibodeau 1998; Case et al. 2004; Dubin 1999; Pagourtzi et al. 2003). Real estate appraisers, local taxation offices, mortgage lenders and insurance companies are eager to know the current value of properties in line with changing market conditions. Models that predict housing values should, thus, be calibrated with most recent sales that represent current developments in the market. There is much demand for models that can detect and predict trends in the market at an early stage, and they require robust predictions while being calibrated with only few observations (Kuntz and Helbich 2014). This is generally problematic in hedonic analysis that may require thousands of transactions to deliver reliable statistically significant estimates for various structural and spatial attributes that influence housing prices in a particular market.

Assessment of a value at risk is also an important part of cost-benefit analyses (CBA) in the context of natural hazards and risk mitigation policies. Valuation of capital at risk is an essential part of the direct damage estimate in any CBA and provides a tool for policy makers to efficiently allocate resources among competing risk management options. Flood risk is one of the most frequently occurring disasters worldwide, and CBA is widely applied to assess flood management strategies (Gamper et al. 2006; Hall et al. 2005; Merz et al. 2010; Penning-Rowsell et al. 2005; Hallegatte 2006). Usually CBA's for flood risk rely on combining geographic (GIS) maps with flood zones (with probabilities and potential inundation depths), damage functions and land use data (Dutta et al. 2003; Hall et al. 2003; Hall et al. 2005). Flood risk is the sum of total impacts and probabilities of flood events with a particular severity and inundation depth:

Flood risk $=\sum_{i=1}^{i_{\max }} P\left(X_{i}\right) * D\left(X_{i}\right) * K$ 
Where $X$ is a list of all possible flood scenarios, $P(X)$ a list of all related probabilities, $D(X)$ is the damage to a property as a function of inundation depth, water stream speed and salinity (often expressed as a percentage of a property destroyed), and $K$ is the market value of properties located within the flood zone. Given the growing concerns for increasing vulnerability of urban areas driven by climate change and a need for climate adaptation policies, a majority of the studies focus either on calculating new probabilities ( $P(X)$, Eq. 2.I) (Hirabayashi et al. 2013; Ward et al. 2014) or on estimating damage functions $(D(X)$, Eq. 2.I) for properties and infrastructure (Farber 1987; Oliveri and Santoro 2000, Merz et al. 2010). Thus, while a lot of attention goes to clarifying location-specific hazard probabilities and relations between severity of hazards and corresponding damages to properties, the value of capital at risk is assumed to remain static. Possible structural changes in property markets driven by, for example, increasing severity and frequency of flooding, are not considered. This approach is insufficient in a changing environment, especially when climate-related natural hazards are concerned. While little attention is currently given to changes in capital in hazard zones ( $K$, Eq. 2.I), several hedonic studies documented that flood risk premium is not stable over time. Namely, values of flood-prone properties drop significantly after a flood event, but recover back just after a few years (Atreya and Ferreira 2012; Bin and Landry 2013; Pryce et al. 2009). It appears that recent experience with flooding awakens or reinforces the perceived risks and costs associated with flooding, and that a lack of flooding experience vanishes these perceptions. Thus, flood risk assessments in CBA may be quite sensitive to the timing when a flood discount is measured or to the year of a property valuation. It is important to keep track of these market responses to floods driven by exogenous shocks and changes in individual risk perceptions and location choices, and to update the expected prices and the corresponding value of the capital at stake.

Hedonic analysis is commonly used to asses and predict property prices and to estimate the flood risk premiums. In hedonic analysis a list of housing attributes is combined into a multiple regression with sales price as dependent variable. It can be used to predict future sales prices, yet the main purpose of these models is to calculate the marginal implicit price of specific housing attributes such as neighbourhood amenities, environmental quality or safety against floods (Atreya and Ferreira 2012; Bin and Polasky 2004; Bin and Landry 2013; Hallstrom and Smith 2005). Hedonic studies often employ a large scale cross-sectional data measured within a long time frame. The question remains whether these models can effectively predict prices when calibrated with only few recent sales. One of the problems with assessing and predicting future sales prices using traditional hedonic models, is the chosen functional relation between spatial factors and sales prices. The fact that 
housing location has a strong effect on sales price is widely acknowledged, but the complexity of space as a factor is not captured well enough in the hedonic literature (Dubin 1992). There are several ways to construct regression models that account for spatial and neighbourhood characteristics, including for example spatial error model (Anselin 200I). An extensive analysis of out-of-sample prediction performance of various spatial (econometric) models has been performed by Voltz and Webster (1990), Bourassa et al. (2007) and Basu and Thibodeau (1998). While usually hedonic analysis (including spatial error models) performs well on large multi-year datasets, there is a need for an improved approach for robust assessment of property prices in highly dynamic markets. As discussed above, dramatic prices changes in property market suffering from a shock, such as flooding for example, require a price prediction model that can work on small samples such as a few months of transaction data.

To mitigate the problem of a careful and robust assessment of the influence of spatial factors, Dubin (1992) suggests to omit all spatial variables in the hedonic analysis and to interpolate the spatial correlation in property prices by using kriging. Kriging is a spatial statistics method used to perform spatial interpolation, and is used for a wide range of applications in environmental sciences also based just on few observation points (Alemi et al. 1988; Delhomme 1978; Hernandez-Stefanoni and PonceHernandez 2006; Webster and Burgess 1983). Yet just a few hedonic studies have adopted this method despite the fact that it can significantly improve the prediction performance compared to the traditional regression-based hedonic analysis (Case et al. 2004; Kuntz and Helbich 2014). Some studies applied the technique to correct for spatial autocorrelation (Basu and Thibodeau 1998; Bourassa et al. 2007; Militino et al. 2004), and other studies also validated the method through out-of-sample predictions (Case et al. 2004; Kuntz and Helbich 2014). The model specifications examined in this study are based on hedonic analysis and kriging. While the literature suggests that kriging improves the prediction performance of spatial hedonic models, the performance of these models over a range sample sizes have yet to be tested. Therefore, the main purpose of this paper is to assess the robustness of the prediction performance of spatial hedonic models, either enhanced or not with kriging, under different sample sizes.

Another method used to predict property prices is artificial neural networks (Nguyen and Cipps, 200I). While housing attributes in hedonic models are typically fitted with linear, log or squared relationships with price, artificial neural networks are used to fit more complex functional relationships. This works well with large housing transactions samples, but is sensitive to over-fitting when calibrated with 
small samples. Nguyen and Cipps (200I) have compared the performance of multiple regression models with artificial neural network models across sample sizes, and have concluded that the multiple regression models perform better than artificial neural network models at small sample sizes. Moreover, regression models are far less complicated and more widely used than artificial neural networks, thus we do not consider it further in our paper.

Given the number of different methods to assess and predict property prices, the purpose of this study is to understand which model can be widely applicable across a range of sample sizes and can be quickly calibrated in a changing environment. Our main research objective is to determine which specification of a spatial hedonic model is the best in predicting sales prices when calibrated with a small sample of recent sales. We test various hedonic models by systematically changing the size of the in-sample set of transactions based on which the models are calibrated. The sales prices of out-of-sample properties are predicted with the calibrated models. We perform this analysis on the dataset with residential property transactions between 1992 and 2002 in a housing market in North Carolina. We also analyse the reliability of the flood risk discount assessed with different statistical models under various insample sizes. Given the challenges of assessing capital at risk and flood risk discount in particular in a changing environment, the outcomes of the current paper may be of interest for policy makers conducting CBA of flood risk management policies, for monitoring developments in insured and uninsured property values and capital-atrisk, and for assessing structural changes in property markets in response to natural hazards. The analysis in this paper can be applied to a wide range of natural hazards and real estate appraisal in general. Our results demonstrate when and why the kriging-enhanced hedonic model performs better. Especially the prediction performance with small sample sizes is interesting, because this is where the model can quickly be calibrated and be applied for price predictions under changing market conditions. We present the specific improvements to the traditional spatial hedonic model that enhance the prediction accuracy, especially when it is calibrated with few observations.

The paper proceeds as follows. We start by giving a description of the data and the four different models, which are systematically compared for different samples sizes. Then, we explain how the analysis is done to compare the models. We conclude by discussing the results and their implications. 


\subsection{Methods}

\subsection{Data}

We use housing sales data in Pitt County, North Carolina, from January 1992 to June 2002 (Bin and Landry, 20I3) to calibrate the models and to validate their prediction performance'. The area provides an excellent natural experiment setting for this study in that it had enjoyed a period of relative calm, not experiencing major hurricane flooding since Hurricane Hazel in 1954, followed by two major hurricanes. Hurricane Fran (1996) produced millions of dollars in property damages resulting from profuse rainfall, flash floods, and severe storm surge. Three years later Hurricane Floyd (1999) brought torrential rains and record flooding which resulted in one of the largest peacetime evacuations in U.S. history (Bin and Polasky, 2004). The data include information on sales price, property characteristics such as age and size, dummy variables that represent the presence or absence of extra facilities of the property, spatial information on the distance to amenities and disamenities, flood zoning, and time of sales. The summary statistics of all the relevant property characteristics can be found in Table 2.I.

Table 2.I. Summary statistics of the property attributes

\begin{tabular}{lll}
\hline Variables & \multicolumn{2}{l}{ Summary $(\mathbf{N}=4779)$} \\
\cline { 2 - 3 } & Mean & $\begin{array}{l}\text { Standard } \\
\text { deviation }\end{array}$ \\
\hline Sales price & USD I56 6I2 & 87354 \\
Age of the house & 22.4 years & 19 \\
Number of bedrooms & 3.2 & 0.59 \\
Total structure square feet & $239 \mathrm{I}$ & 993 \\
Lot size in acres & 0.64 & 2.4 \\
Gas heating $(=I)$ & 0.35 & 0.48 \\
Fireplace $(=I)$ & 0.77 & 0.42 \\
Face brick $(=I)$ & 0.48 & 0.50 \\
Hard wood flood $(=I)$ & 0.25 & 0.43 \\
Good quality $(=I)$ & 0.031 & 0.17 \\
Vacant home $(=I)$ & 0.0048 & 0.069
\end{tabular}

\footnotetext{
I Note, that the original study of Bin and Landry (2013) employed a spatial error model. Kriging and spatial error models differ in the way in which the spatial weight matrix is constructed. The spatial weight matrices in spatial error models are constructed based on the assumptions of the user, whereas in kriging they are based on the spatial structure of the error, which is defined in the construction of the semivariogram. The spatial error model is particularly relevant for proper estimation of the coefficients in the hedonic price estimation, whereas kriging focuses on the prediction of the dependent variable.
} 


\begin{tabular}{lll} 
Distance to creek & 854 feet & 596 \\
Distance to airport & 33966 feet & 17859 \\
Distance to major road & 135 feet & 99 \\
Distance to business centre & 4632 feet & 2452 \\
Distance to railroad & $5498 \mathrm{feet}$ & 6378 \\
Distance to Tar River & $20999 \mathrm{feet}$ & 17587 \\
Distance to park & $7490 \mathrm{feet}$ & $705 \mathrm{I}$ \\
Sold between Fran and Floyd $(=\mathrm{I})$ & 0.34 & 0.48 \\
Sold after Floyd $(=\mathrm{I})$ & 0.37 & 0.47 \\
Floodplain $(=\mathrm{I})$ & 0.064 & 0.24 \\
\hline
\end{tabular}

\subsubsection{Model specifications}

The hedonic price function of a property is given by

$\ln P^{i}=\beta_{0}+\sum_{k=1}^{K} \beta_{k} x_{k}^{i}+E^{i}$

where $\ln P^{i}$ is the natural $\log$ of the sales price of property $i, \beta_{0}$ is the intercept, $\beta_{k}$ is the coefficient for each property characteristic $k, x_{k}^{i}$ is the value of characteristic $k$ of property $i$, and $E^{i}$ is the residual of the predicted property price. Our objective is to identify the model that provides the smallest prediction errors in the out-ofsample predictions for various sample sizes. The models to be compared include:

- A spatial hedonic model from Bin and Landry (2013)

- $\quad$ An adjusted version of MI with different functional forms

- $\quad M 2$ with a reduced number of input variables

- $M 3$ whereby spatial variability in property prices is predicted with kriging (M4)

The hedonic analysis is used to estimate the coefficients of the input variables $\beta_{k}$ (Eq. 2.2). In M4 hedonic analysis is used to understand the influence of the core spatial variable of interest (flood risk) on property prices while the rest of the spatial variability in prices is captured by interpolating the residuals $\left(E^{i}\right.$, Eq. 2.2$)$ using kriging. A list of all the input variables can be found in Table 2.2. MI is same model with the same specifications as used in Bin and Landry (20I3). However, the model in Bin and Landry (2013) has more dummy variables than $\mathrm{MI}$ in this paper, as they distinguish the 100-year and 500-year flood zones. The reason this separation is not made in $\mathrm{MI}$ is that only $2 \%$ properties in the 500 -year floodplain were sold, so that the 500 -year floodplain properties are many times absent in this subset. Therefore, we merge the 100 -year and 500-year flood zone properties in one floodplain variable. 
Improved Methods for Predicting Property Prices in Hazard-prone Dynamic Markets

Table 2.2. Input variables and their functional forms for the hedonic models ${ }^{2}$

\begin{tabular}{lllll}
\hline Variables & MI & M2 & M3 & M4 \\
\hline Age of the house & $\mathrm{X}+\mathrm{X}^{2}$ & $\mathrm{X}+\sqrt{\mathrm{X}}$ & $\mathrm{X}$ & $\mathrm{X}$ \\
Number of bedrooms & $\mathrm{X}+\mathrm{X}^{2}$ & $\mathrm{X}+\sqrt{\mathrm{X}}$ & $\mathrm{X}$ & $\mathrm{X}$ \\
Lot size in acres & $\mathrm{X}+\mathrm{X}^{2}$ & $\mathrm{X}+\sqrt{\mathrm{X}}$ & $\sqrt{\mathrm{X}}$ & $\sqrt{\mathrm{X}}$ \\
Total structure square feet & $\mathrm{X}+\mathrm{X}^{2}$ & $\mathrm{X}+\ln (\mathrm{X})$ & $\ln (\mathrm{X})$ & $\ln (\mathrm{X})$ \\
Gas heating (=I) & $\mathrm{X}$ & $\mathrm{X}$ & & \\
Fireplace (=I) & $\mathrm{X}$ & $\mathrm{X}$ & & \\
Face brick (=I) & $\mathrm{X}$ & $\mathrm{X}$ & & \\
Hard wood flood (=I) & $\mathrm{X}$ & $\mathrm{X}$ & & \\
Good quality (=I) & $\mathrm{X}$ & $\mathrm{X}$ & & \\
Vacant home (=I) & $\mathrm{X}$ & $\mathrm{X}$ & & \\
Log of distance to creek & $\mathrm{X}$ & $\mathrm{X}$ & & Kriging \\
Log of distance to airport & $\mathrm{X}$ & $\mathrm{X}$ & & Kriging \\
Log of distance to major road & $\mathrm{X}$ & $\mathrm{X}$ & & Kriging \\
Log of distance to business centre & $\mathrm{X}$ & $\mathrm{X}$ & & Kriging \\
Log of distance to railroad & $\mathrm{X}$ & $\mathrm{X}$ & & Kriging \\
Log of distance to Tar River & $\mathrm{X}$ & $\mathrm{X}$ & & Kriging \\
Log of distance to park & $\mathrm{X}$ & $\mathrm{X}$ & & Kriging \\
Sold between Fran and Floyd $(=I)$ & $\mathrm{X}$ & $\mathrm{X}$ & & \\
Sold after Floyd (=I) & $\mathrm{X}$ & $\mathrm{X}$ & & \\
Floodplain (=I) & $\mathrm{X}$ & $\mathrm{X}$ & $\mathrm{X}$ & $\mathrm{X}$ \\
Floodplain * sold btw Fran and Floyd & $\mathrm{X}$ & $\mathrm{X}$ & & \\
Floodplain * sold after Floyd & $\mathrm{X}$ & $\mathrm{X}$ & & \\
\hline
\end{tabular}

M2 was built with the same input variables as MI, while changing some of the functional forms in order to better describe the saturation behaviour of the variable's influence on price. The variables bedrooms ${ }^{2}$, age ${ }^{2}$, square footage ${ }^{2}$ and acres $^{2}$ were substituted by $\sqrt{\text { bedrooms }}, \sqrt{\text { age }}, \ln$ (square footage) and $\sqrt{\text { acres }}$ respectively. These variables often enter the hedonic analysis function in the quadratic specification (Case et al. 2004). Yet, in our dataset price dependence on them does not necessary follow the parabolic form (Do and Grudnitski, 1993; Goodman and Thibodeau, 1995) (Fig. Al, Appendix 2.a). In M3 and M4 we consider a reduced regression that contains only the main characteristics of the properties - sq. footage,

\footnotetext{
2 A variable 'Schools' has not been included in the analysis since the school quality in this particular areas is rather homogeneous. Furthermore, school rating does not have statistically significant effect on property prices.
} 
bedrooms, acres and age - that have a clear, straightforward and always statistically significant effect on price. ${ }^{3}$

In M4, the kriging procedure compliments the hedonic analysis to explain spatial correlation in property prices. The hedonic regression of $M 3$ does not contain spatial characteristics of the property. All spatial relations and neighbourhood effects are captured by the residuals in predicted property prices, $E^{i}$ (Eq. 2.2). Therefore, $E^{i}$ in the out-of-sample prediction is added as a function of the in-sample residuals $E^{j}$ of nearby properties (Eq. 2.3). This function can be written as

$$
\begin{aligned}
& E^{i}=\sum_{j=1}^{N} W^{i, j} E^{j}, \\
& \sum_{j=1}^{N} W^{i, j}=1
\end{aligned}
$$

whereby $W^{i, j}$ is a spatial weight matrix which specifies how much the residual price of an in-sample property $j$ affects the residual of an out-of-sample property $i$, and depends on the spatial distance between property $i$ and property $j$. The spatial weight matrix is derived from a model of variance as a function of distance, called a semivariogram. The semivariogram is constructed by calculating the variance in $E$ for all point pairs within a certain distance class. It is expected that this variance increases when the distance between properties increases, but that it levels off with increasing distance. This relation is fitted with an exponential function, which is used to specify the spatial weight matrix; nearest properties get the highest weight. The user can specify how many of the nearest properties are taken into account in the kriging interpolation, which is a trade-off between computation time and prediction accuracy (see Alemi et al. 1988 and Delhomme 1978 for more details on the kriging procedure). In our case we take the 15 nearest properties ${ }^{4}$.

\subsubsection{Analysis}

We split the entire data set (4779 observations) into in-sample and out-of-sample parts. The coefficients of the hedonic regressions are calculated using in-sample data, which is constructed as a subset of the sales data ranging from $0.1 \%$ to $20 \%$. We

\footnotetext{
3 We did a thorough analysis with various combinations of log and square root functional forms to identify the functional forms that best fitted the transaction data.

4 We did a sensitivity analysis and concluded that 10-20 nearby properties is a good number to use since it does not change the model's performance. More than 20 does not change the performance but enhances computation time, while less than 10 properties reduces the prediction performance. The results of our sensitivity analysis are available upon request.
} 
systematically vary this share to understand how small the in-sample subset can be to be able to deliver an acceptable predictive power for each of the models. The remaining transactions form the out-of-sample dataset, which we use to compare the prices predicted by the four models against the actual sales price. The coefficients of the in-sample hedonic regressions are used to form the predicted prices of the out-of-sample properties in MI, M2 and M3. In M4 we sum the regression estimates and the kriged residuals.

Further, we apply Monte Carlo method by taking 50000 random subsets ranging from $0.1 \%(\mathrm{~N}=48)$ to $20 \%(\mathrm{~N}=956)$ of the dataset, with constant density ${ }^{5}$. Each model is calibrated with the same subsets, so that we can make pairwise comparisons. There is no limit to the number of subsets we can take, so we decide to take enough to cover the full range of model performances. The performance of a model may strongly depend on the subset it is calibrated on. Therefore we choose to look not only at average performance, but also at the $95 \%$ confidence interval of the performance range across sample sizes.

First, $\mathrm{MI}$ and $\mathrm{M} 2$ are compared to assess how changing functional forms affect the prediction performance across sample sizes. Second, M2 and M3 are compared to assess the effect of reducing the number of input variables in the hedonic analysis, which should be more suitable for predicting prices based on small samples. Third, M3 and M4 are compared to see the effect of kriging for different sample sizes. And finally, the performances of all models are compared to see which model performs best across various sample sizes.

For comparing the models' prediction performances, we use the following metrics: Root Mean Squared prediction Error (RMSE) (Bin, 2004; Case et al., 2004; Selim, 2009), Mean Absolute prediction Error (MAE) (Bin, 2004; Case et al., 2004; Selim, 2009), Standard Deviation of prediction Error (SDE) (Case et al., 2004) and Adjusted R-squared (Laurice and Bhattacharya, 2005). We look at the Adjusted $\mathrm{R}^{2}$ of the regression described by

$\ln ($ actual value $) \sim \ln ($ predicted value $)$

which is a measure of the model's explanatory power. RMSE, MAE and SDE are measures for prediction accuracy and precision (see Appendix 2.b).

\footnotetext{
${ }^{5}$ The analysis was done in R, version 3.2.0. Computation time was approximately I hour.
} 


\subsection{Results}

\subsection{Comparing functional specifications of the full hedonic model (MI and M2)}

Comparing $\mathrm{MI}$ and $\mathrm{M} 2$ (Fig. 2.I), the prediction performance of $M 2$ is considerably better than MI, with the strongest effect at small sample sizes. Looking at MAE, we see that the median performance of both models does not differ at sample fractions above $0.10(N>480)$. However, when looking at the metrics RMSE and SDE the median prediction performance of $M I$ is still worse than $M 2$ at samples of $N \approx 240$. RMSE and SDE are performance metrics that 'punish' the model for highly inaccurate predictions. We see that when price assessments for the out-of-sample set need to be made on a rather small in-sample dataset MI produces highly volatile prediction outputs, with price predictions that sometimes deviate from actual sales by several orders of magnitude. The upper limits of the $95 \%$ confidence intervals of MI's performance metrics (Fig. 2.I) show that MI often performs much worse than M2 as a result of these highly inaccurate price predictions.
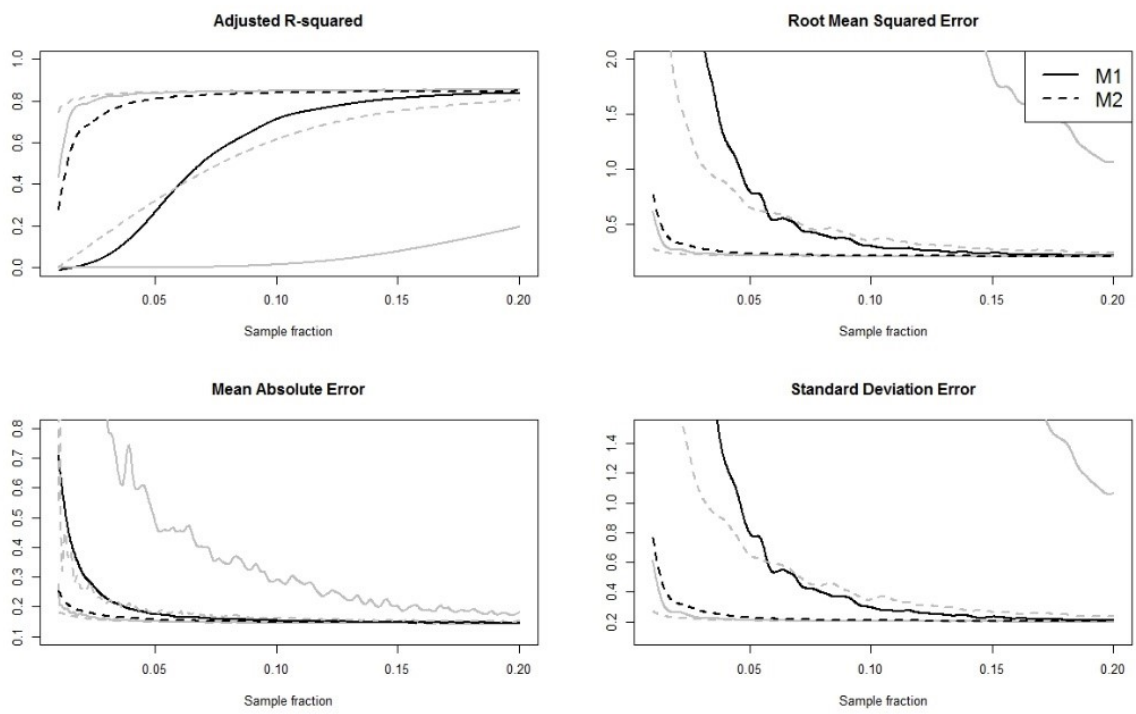

Figure 2.I. Four out-of-sample prediction performance metrics as function of sample fraction, comparing MI (continuous) and M2 (dashed). In black: smoothing spline of the moving-window median. In grey: the $95 \%$ confidence interval boundary.

The difference in performance between $\mathrm{MI}$ and $\mathrm{M} 2$ can be explained by the functional forms of the input variables. The functional forms of some input variables of $\mathrm{MI}$ are described by a squared relation with price, which is meant to represent 
the saturation behaviour of the characteristic's effect on price. However, a squared or second degree polynomial function is not a saturating function. Rather, it has a peak (minimum value or maximum value depending on the sign of the coefficient) and can only approximate saturation behaviour on a local scale, but it can neither describe nor predict actual saturation behaviour overall. This results in a low model fit, which is measured by the Adjusted R-squared metric (Fig. 2.I). When the properties in the subset only contain a limited range of the characteristics compared to their entire range within the population, it can lead to large errors in the predicted price of properties with characteristics on the extreme ends of the range. Changing the squared functions to square root and log functions as in M2 could therefore considerably reduce the extreme prediction errors.

\subsubsection{Reducing the number of explanatory variables (M2 and M3)}

Comparing M2 and M3 (Fig. 2.2) we see that M3 performs consistently better at sample fractions below $0.05(\mathrm{~N}<240)$. The prediction performance of $\mathrm{M} 3$ measured by all 4 metrics is relatively constant across sample sizes, whereas M2's performance reduces drastically with decreasing sample sizes. This shows that a decrease in the number of variables in the hedonic analysis can enhance the model's prediction performance when it is calibrated with few sales. Over-fitting is likely to happen in the case of small sample sizes and large numbers of explanatory variables. In these cases, the explanatory variables that have little explanatory value will not contribute to the prediction performance of the model, but rather start explaining noise within the sample. This results in a loss of generalisation in the model, and an increase in the stochastic behaviour of the predicted values. It is therefore crucial that the hedonic model only takes into account the factors that are of key importance and have a clear and sensible effect on the price, especially when it is based only on few observation data points. 

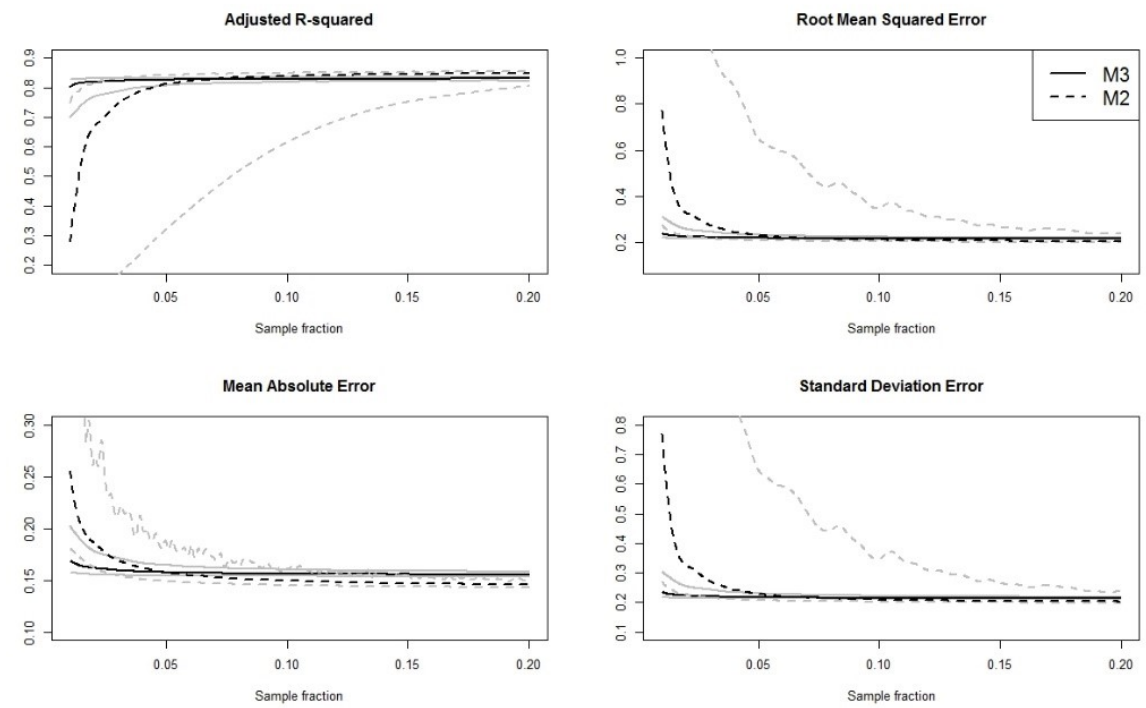

Figure 2.2. Four out-of-sample prediction performance metrics as function of sample fraction, comparing M3 (continuous) and M2 (dashed). In black: smoothing spline of the moving-window median. In grey: the $95 \%$ confidence interval boundary.

At sample fractions higher than 0.05 (or $N>240$ ) $M 2$ generally has a lower mean absolute error than $\mathrm{M} 3$, suggesting that $\mathrm{M} 2$ outperforms $\mathrm{M} 3$ when they are calibrated with samples of $N>240$. However, this conclusion does not hold when looking at the other performance metrics. The upper part of the $95 \%$ confidence interval of RMSE and SDE shows that the predictions of $M 2$ can still be quite volatile compared to $M 3$. Thus even though $M 2$ performs better than $M 3$ on average with samples of $N>240$, the precision of M2's predictions is still lower than that of $M 3$, where $M 2$ has a higher probability of strongly inaccurate predictions.

\subsubsection{Explaining spatial variability in prices through kriging (M3 and} M4)

From the first glance the previous discussion suggests that dropping various spatial factors when explaining property prices may be attractive. However, this is relevant only for small samples and serves as a disadvantage for larger samples. Indeed, properties in the same neighbourhood share similar spatial attributes such as proximity to parks, highways or other transport hubs, shopping centres, schools, and so on. It is a loss not to control for these given data availability. At the same time, the influence of all of them is not really vital for the research question at hand. Often one only needs to zoom in into a few specific attributes explaining price variations in the market and exclude the rest in an attempt to improve hedonic model 
performance. As we have seen above, including too many explanatory factors may jeopardize the latter for relatively small in-samples.

\section{(Semi)variogram residuals}

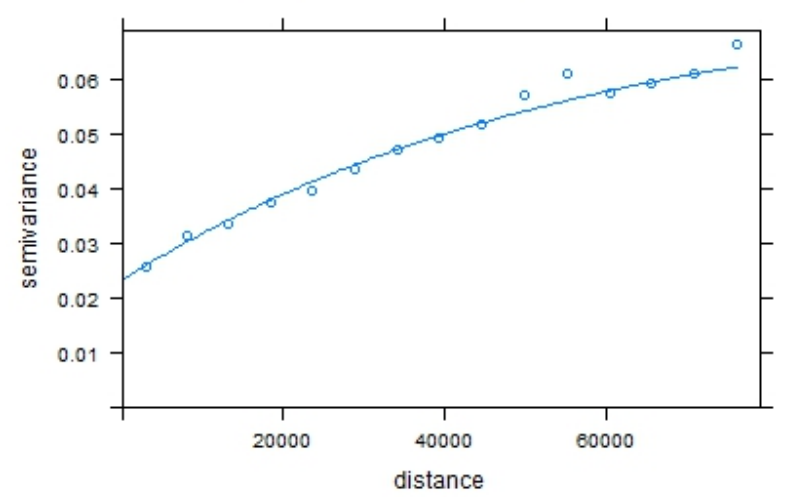

Figure 2.3. Semivariogram of the in-sample residuals of M3. The graph shows that there is spatial autocorrelation in the residuals, since the variance in residuals increases with distance between properties.

Kriging offers an alternative way to account for the influence of spatial complexity in price assessments. Namely, it captures any systematic variation in prices through the analysis of residuals. In our dataset we find a clear spatial correlation in the semivariogram of the in-sample residuals of M3 (Fig. 2.3). The semivariance increases with distance between properties, which shows that property prices are spatially correlated. This indicates that the prediction performance of M3 can be improved with regression kriging (Basu and Thibodeau, 1998). Regression kriging is done with the same variables as the hedonic model M3, after which the residuals are interpolated, so that only the remaining variation is addressed.

Comparing M3 (reduced hedonic model without kriging) and M4 (with kriging), we see that the model's performance consistently improves when kriging captures the spatial autocorrelation in residuals (Fig. 2.4). SDE, MEA and RMSE are consistently lower when kriging is added to M3 (Wilcoxon Signed-Rank Test, $\mathrm{P}<0.00 \mathrm{I}$ ), and Adjusted R-squared is consistently higher with kriging (Wilcoxon Signed-Rank Test, $\mathrm{P}<0.00 \mathrm{I})$. Most importantly, this result is consistent across all sample fractions.

The enhanced performance of M4 with kriging is more pronounced with increasing sample sizes (Fig. 2.4). At small sample sizes the density of the sample is so low that some of the predicted property prices are not spatially correlated with any of the property prices in the sample. The chance of having out-of-sample property prices 
that are spatially uncorrelated with the sample diminishes when the density of the sample increases. This is why the improvement of kriging becomes more pronounced with increasing sample sizes.
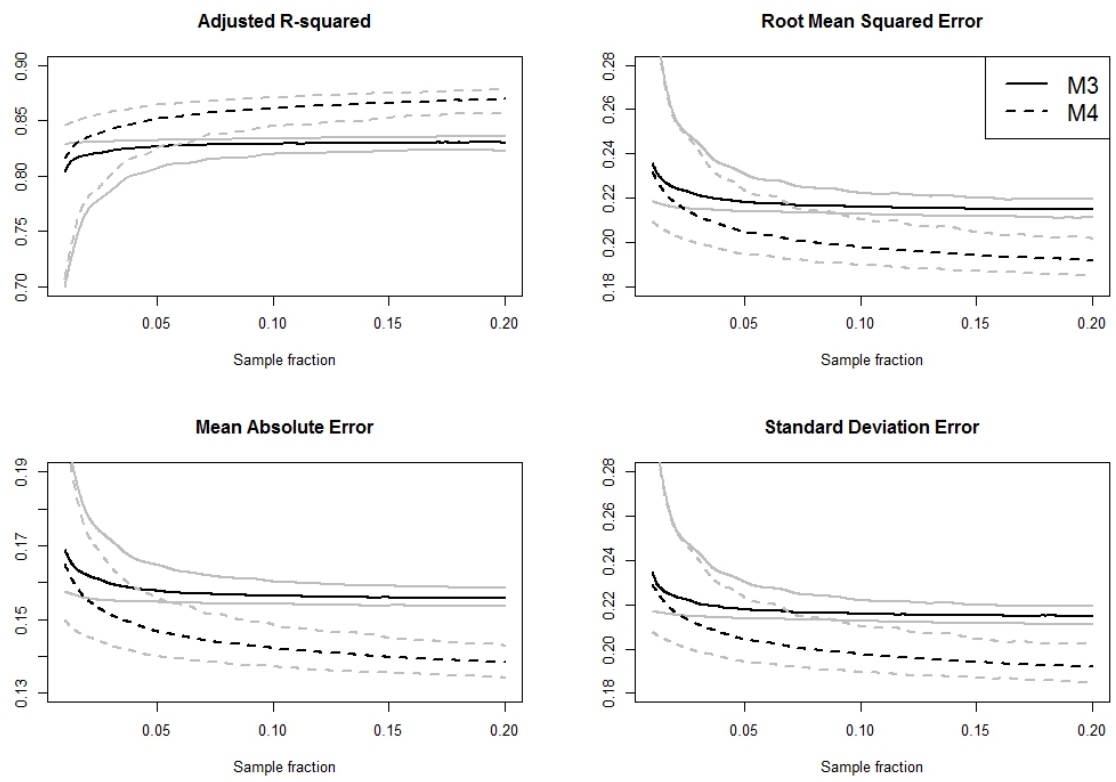

Figure 2.4. Out-of-sample prediction performance of $M 3$ (continuous) and M4 with kriging (dashed) as function of sample fraction. In black: smoothing spline of the moving-window median. In grey: $\mathbf{9 5 \%}$ confidence interval boundary.

When comparing the four models, we find that M4 with kriging performs consistently better across various sample sizes. Thus, it delivers a more robust model to be used in hedonic analysis without a need for a researcher to worry about meeting a particular threshold of an in-sample size: it simply performs well for a large variety of sample sizes. Looking at the performance metrics, M3 with kriging is the best model in $96.2 \%$ of the cases with RMSE and SDE, $97.2 \%$ of the cases with MAE and 98.9\% of the cases with Adjusted R-squared. It also implies that kriging explains the spatial variability in property prices better than the spatial variables that are included in $\mathrm{MI}$ and $\mathrm{M} 2$ and releases a researcher from worrying about functional specifications of the spatial variables of secondary importance. At the same time, using kriging in combination with traditional hedonic analysis allows disentangling spatial attributes of a particular interests for economic analysis - e.g. a location within a flood zone in our case - to be studied with precision. 


\subsubsection{Implications for policy making: example of the flood risk discount}

Outcomes of a hedonic analysis often serve as inputs for a larger CBA. When discussing flood risk management policy, either an adaptive estimation of the capital at stake (i.e. overall property price assessment) or a particular value of a flood risk discount (i.e. a value of the regression coefficient for a flood-zone dummy) plays a major role in the estimation of costs and benefits of a particular measure. Yet, what does the sensitivity to the in-sample size imply for a CBA? Let us examine the flood risk discount in particular (Table 2.3). It must be noted that the flood-zone dummy in $\mathrm{Ml}$ and $\mathrm{M} 2$ is also represented by two interaction terms (between hurricanes Fran and Floyd, and after hurricane Floyd) to see how the flood coefficient changes over time. Thus, the flood risk coefficient for $\mathrm{MI}$ and $\mathrm{M} 2$ in Table 2.3 was controlled for the interaction terms. Table 3 presents the averages across the 50,000 Monte Carlo sampling runs.

The full regression model with the traditional functional specification $\mathrm{MI}$ provides a rather unstable estimation of the flood risk coefficient since it varies greatly with the sample size. In fact for small in-sample sizes the flood dummy coefficient is positive but its standard deviation across random Monte Carlo sampling sets is huge undermining its statistical insignificance. The same can be said about $M 2$ for small insample sizes, although the standard deviation is already much lower than MI. M3 and M4 have the lowest standard deviation for small in-sample fractions $(<0.05)$. With larger sample fractions $(>0.10)$ it does not matter anymore which model is used to predict the flood coefficient.

Table 2.3. Stability of a regression coefficient of the floodplain variable over various insample sizes

\begin{tabular}{lllllll}
\hline & M1 & \multicolumn{3}{c}{ M2 } & \multicolumn{3}{c}{ M3 \& M4 } \\
\cline { 2 - 7 } Sample fraction & mean & SD & mean & SD & mean & SD \\
\hline$<0,05$ & 0.150 & 17.034 & -0.055 & 0.447 & -0.050 & 0.109 \\
$<0,10$ & -0.063 & 0.103 & -0.061 & 0.056 & -0.052 & 0.055 \\
$<0,15$ & -0.061 & 0.040 & -0.061 & 0.041 & -0.053 & 0.041 \\
$>0,15$ & -0.060 & 0.033 & -0.060 & 0.033 & -0.053 & 0.033 \\
\hline total population & -0.056 & - & -0.056 & - & -0.054 & - \\
\hline
\end{tabular}


Results in Table 2.3 suggest that the sales price differential between inside and outside the floodplains ranges from $5.0 \%$ to $6.3 \%$ with an exception of $\mathrm{MI}$ with less than 0.05 sample fraction. Several previous studies have documented the price reduction from location in a floodplain (MacDonald, Murdoch, and White 1987; Bin and Polasky 2004; Hallstrom and Smith 2005; Bin, Kruse, and Landry 2008; Daniel, Florax, and Rietveld 2009). A common finding in these studies is that location within a floodplain lowers property value anywhere from four to twelve percent. As shown in Table 2.3, our approach to limit the number of variables that enter the hedonic regression can be quite useful in determining the risk premiums associated with flooding especially with small samples. Our results may help insurance practitioners and policy makers make informed decisions on the flood risk management especially when the available data set is very limited.

\subsection{Discussion}

Across all sample sizes we see that M4 with kriging performs best in the out-ofsample predictions regardless of the in-sample size. This model differs from $\mathrm{MI}$ and M2 in the way the spatial variables enter the price estimation. In M3 the spatial variables were omitted completely, whereas kriging was used in M4 to predict spatial variability and spatial autocorrelation in property prices by analysing the residuals. Kriging-based M4 is thus more powerful in predicting the spatial patterns in property prices. This may not be surprising, as kriging is used for spatial interpolation by explicitly accounting for spatial autocorrelation, which is often present the property market (Basu and Thibodeau 1998; Bourassa et al. 2007; Dubin 1992; Militino et al. 2004). Case et al. (2004) have already shown that kriging can, for this reason, enhance the out-of-sample prediction performance of spatial hedonic models. Yet, their models were calibrated with very large samples $(\mathrm{N} \approx 50,000)$ and the question remained whether these conclusions hold for small samples. We have specified a model that is consistent in assessing housing prices and predicting future sales prices, even when calibrated with a limited number of recent sales. We can zoom into the mechanisms of why this model performs best by comparing MI, M2, M3 and M4 with kriging pairwise across different sample sizes.

Comparing $\mathrm{MI}$ and $\mathrm{M} 2$ reveals that the squared terms in the hedonic model can cause large errors in out-of-sample predictions of the property prices, with estimated prices that sometimes deviate even by several orders of magnitude from the actual price. This problem especially occurred at small sample sizes for which $\mathrm{MI}$ was calibrated. The poor out-of-sample prediction performances were expressed by a 
high variability in prediction errors and a low model fit. The latter indicates that the chosen functional forms of the variables may not represent their actual effect on price. Squared functional forms, and sometimes even cubed functional forms, are currently widely used in hedonic literature (Bin and Landry 2013; Case et al. 2004). These models are based on datasets that are usually large enough to sanitise the effects. Yet, we found that even with samples of $N=900$ the predictions can be inaccurate in the out-of-sample predictions as a result of a low model fit.

A comparison between $M 2$ and $M 3$ reveals that over-fitting is the main cause of the poor out-of-sample prediction performance of $\mathrm{M} 2$ at very small sample sizes. $A$ similar mechanism called over-training is also causing poor prediction performances of artificial neural network models (Nguyen and Cripps, 200I). Reducing the number of input variables in $\mathrm{M} 3$ results in a consistent prediction performance across sample sizes. Moreover, it leads to a decrease of volatility in the model's predictions. However, we also observe that M2 scores better on the metric mean absolute error when a sample size increases. This implies that some of the model's parameters can only be estimated when the number of sales is high. When trying to calibrate a model based on only a few recent sales, it is better to focus only on a few explanatory variables.

M4 with kriging outperforms M3 across the range of sample sizes, but performs only slightly better than $M 3$ with sample sizes of $N \approx 50$. In fact, the improvement of kriging with respect to the prediction performance of $M 4$ increases with sample sizes. The strong prediction performance of $\mathrm{M} 3$ and $\mathrm{M} 4$ at small sample sizes is mainly due to the reduction of variables in the hedonic model, whereas the role of kriging in improving the prediction performance of M4 becomes more important with increasing sample sizes. The latter is caused by an increase in density of the sample, so that the 15 nearest properties that are selected for spatial interpolation are on average closer to the predicted location, i.e.: their actual transaction prices are more correlated with the predicted price. To summarize: we observe that changes in functional forms of the input variables, a reduction of input variables and kriging improve the prediction performance at different parts of the range of sample sizes. Together, these specifications complement each other to form a model that is consistently better in prediction performance across sample sizes.

Despite that M4 consistently produces the best price predictions across a range of sample sizes, we do not suggest that it can replace the hedonic analysis when it is used for other purposes, namely to assess marginal implicit prices of specific housing attributes (Janssen et al. 200I). In this case the spatial attributes of interest should be kept in the hedonic function part of the analysis while the impact of other spatial 
neighbourhood attributes on price may be captured by kriging. The limitation of kriging is that all involved spatial attributes go into the black box of spatial interpolation. Thus, one cannot trace back which spatial factors exactly affect property prices and to what extent.

For the purpose of predicting property values, for example in real estate appraisal (Pagourtzi et al. 2003), it can be useful to work with models that are consistent in their performance even when calibrated with few current sales. Our model, which performs consistently well across a range of sample sizes, is particularly useful for this application. For policy makers that deal with management of natural hazards it is important that a good assessment of the capital at risk is made. Housing markets are affected by macro-economic changes as well as changes in consumer preferences, incomes and WTP for various property attributes. Housing markets in hazard areas experience structural changes in price trends after disastrous events, and these changes are expected to accelerate with climate change. Thus, when conducting a CBA for flood risk management policies it is essential that the assessment of capital at risk or a flood risk premium is based on the most recent sales to better reflect current market conditions. For example when dealing with flood risk, it is important to account for changing risk perceptions, which is driven by flood events and changing flood probabilities, influencing how risk is capitalized into property prices (Pryce et al., 201 I; Atreya and Ferreira, 2012; Bin and Landry, 20I3). Our algorithm allows for a rapid updating of property price assessments and predictions. Therefore, it can quickly capture a market response to potential changes in location preferences, market conditions and flood risk perceptions. This approach can be used to monitor price changes in risk-prone areas, accounting for changes in flood risk and at the same time controlling for autonomous market responses to flood risk. 


\section{CHAPTER 3 - Bridging the Gap Between Revealed and Stated Preferences in Flood-prone Housing Markets}

This chapter is published as a journal article:

de Koning, K., Filatova, T., \& Bin, O. (2017). Bridging the Gap Between Revealed and Stated Preferences in Flood-prone Housing Markets. Ecological economics, 136, I13.

Abstract. This papers attempts to shed light on the gap between revealed and stated preferences methods in flood-prone housing markets. We quantitatively explore the market-level consequences of various behavioural models' assumptions regarding risky choices at the individual level. In addition, we quantify the effect of the marketlevel bias in risk valuation that individual preferences for neighbourhood quality imply. We apply an economic agent-based housing market model to quantify and disentangle the impacts of behavioural biases in individual decisions under risk as well as neighbourhood externalities, which both are known to distort markets. We compare three different micro-level behavioural risk models: expected utility, prospect theory and risk negligence. The flood risk discount on a market level varies significantly, but the results do not rule out any of these models. In general, markets with prospect theory traders overprice risks causing an unrealistically high price differential between risk-prone and risk free properties. The marginal implicit price for flood risk is much closer to empirically found prices in the expected utility and risk negligence models. Additionally we find that individual preferences for neighbourhood quality and multicollinearity in housing attributes can cause a bias in

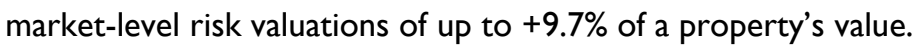
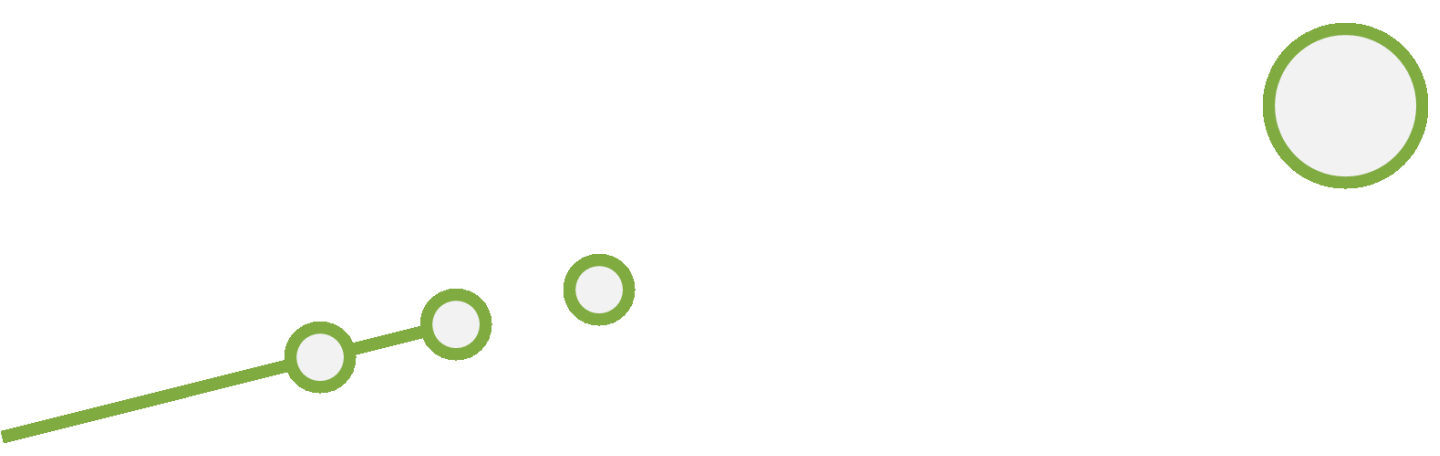



\section{II Introduction}

The price of risk is an important indicator that can facilitate decisions in any risk mitigation policy, which demands for methods to value the social costs of risk as accurately as possible. In particular, in flood risk management the central number that influences the balance between costs and benefits is the price of flood risk ${ }^{6}$. There are two main approaches to assess this value for households (Freeman et al. 2014): (I) the revealed preference method through hedonic pricing of risk (MacDonald et al. 1987; MacDonald et al. 1990; Speyrer and Ragas 1991; Harrison et al. 200I) and (2) the stated preference method through surveys and choice experiments (Mitchell and Carson 1989; Botzen and van den Bergh 20I2). It is claimed that both methods have their own advantages and disadvantages, suggesting that they are comparable in terms of what they measure (Whitehead et al. 2008). Moreover, stemming from the same conceptual roots, theoretically they should be producing similar estimates (Timmermans et al. 1994). Yet, there are consistent differences between the two methods over various applications, also outside flood risk management (Earhart 1998). Economists have been long debating on the reasons behind this gap (Wardman 1988; Adamowicz et al. 1994; Adamowicz et al. 1997; List and Gallet 2001; Cameron et al. 2002; Murphy et al. 2005). There are a number of key factors that cause divergence in stated and revealed preferences. Namely, revealed preferences methods may suffer from collinearity in input variables (Adamowicz et al. 1994; Earhart 1998), from inefficiency in accounting for uncommon attributes (Earhart 1998, Earhart 200I), and from the fact that choices are confined by what is available on the market (Timmermans et al. 1994). At the same time, the stated preferences methods may fail to reflect actual behaviour due to a hypothetical bias (Adamowicz et al. 1994, Murphy et al. 2005) and the fact that people find it difficult to separately express their preferences for individual attributes (Timmermans et al. 1994).

Considering the limitations of both methods, there is a clear benefit in confronting the two approaches in the same settings. Empirical research that used various valuation techniques to elicit willingness to pay or to accept (WTP or WTA) for amenities or disamenities provide the estimates that sometimes differ in orders of magnitude. A small number of studies have attempted to combine the two

\footnotetext{
${ }^{6}$ The price of flood risk serves as an indicator for the social WTP for risk-mitigation policies. For the purpose of CBA stated and revealed preferences methods estimate the discount in housing prices due to flooding risk. For example, what is the marginal price of a certain unit of risk, and thus what is the associated benefit of mitigation strategies that reduce the risk by a particular number of units.
} 
approaches in risk assessments in particular, e.g.: Florax et al. (2005) on pesticide reduction and de Blaeij et al. (2003) on road safety. Furthermore, while the drivers of divergence between both methods are largely known, the question remains to what extent the two approaches quantitatively affect the balance between costs and benefits in risk assessments. This paper is driven by a desire to quantitatively explore the path from individual economic choices, i.e. the level at which stated preferences are collected, to market outcomes, which are used as an input for revealed preferences analysis. We do this through computational modelling of a housing market encompassing floodplains under various behavioural risk models, heterogeneity and interactions. For this we apply an agent-based model (ABM), a method that is particularly strong at linking micro-level choices to the aggregated consequences of many individual decisions including market level impacts (Tesfatsion 2006; Farmer and Foley 2009). This paper serves as the first step in unravelling the interplay of various biases on the gap between the two estimation techniques with a special focus on the value of simulation techniques in this analysis. Thus, we highlight only on two aspects - violations from the expected utility framework and spatial correlation of property attributes that may confuse risk assessments. The paper's aim is to quantitatively explore (I) the market-level consequences of various behavioural models' assumptions regarding risky choices at the individual level, and (2) the effect of the market-level bias that individual preferences for neighbourhood quality imply. By pursuing this dual goal, we contribute to understanding the gap between individual choices, on which stated preference methods rely, and observed market outcomes based on revealed preferences methods. We consider three types of behavioural biases that are known to distort markets: subjective weighting of probabilities, loss aversion, and correlation between amenities and risks.

We proceed with an overview of confounding factors that affect flood-risk analysis on the micro and macro level. Then, we dive more into the details on how ABMs can alleviate the impact of these confounding factors and combine the strengths of revealed and stated preference approaches. The paper proceeds by briefly describing the model and the data that is used for its calibration and validation. We discuss the different sub-models that are systematically compared in the results section. Next, we explain how the analysis is done, and we conclude by discussing the results and their implications. 


\subsection{Individual choices in the presence of risks and amenities}

In the social and economic literature there are several models conceptualizing how people make decisions when facing a risky choice. They range from purely objective judgements about risks by perfectly-rational agents under the conditions of full information (Herstein and Milnor 1953; Von Neumann and Morgenstern 2007) to models that embrace the idea that risk judgements are subjective and biased (Kahneman and Tversky 1979) to more cognitively complex psychological models (Finucane et al. 2000). These models deal with risk perception and decision making in a different way, which leads to different hypotheses about economic choices in a risk situation. Thus, each model will have a different impact on the price differential between risk-prone and risk-free properties. We use the various assumptions for risk-decision strategies based on empirical data and existing theoretical models, and compare aggregated market outcomes emerging from interactions of heterogeneous households in a simulated market. In particular, we test three individual riskperception models to quantify a hedonic price function under the assumptions of three behavioural models: risk negligence, expected utility and prospect theory.

Risk negligence: some surveys for households in hazard-prone areas suggest that the households' decisions to buy a property are made independent of risk (e.g.: Burningham et al. 2008, Willis et al. 20I I). This does not imply that people are always unaware of the hazard, but rather that this knowledge does not affect their decision making. Willis and colleagues (20II) interviewed people who live in a flood zone, and most of them acknowledge the fact that they live in a flood risk zone. However, they do not worry about the flood in their everyday lives. Thus, despite that they perceive the risk as real, it does not affect their behaviour and their choices in buying a house. When explicitly asked for in an interview, people may state that they have been aware of the flood risk, but it does not imply that it affected their weighing of costs and benefit of living in the hazard zone (Burningham et al. 2008, Willis et al. $20 \mathrm{II})$. Another set of empirical literature indicates that people residing in floodprone areas may just have biased risk perceptions. In other words, they either do not realise that they bought a property in a flood zone (Chivers and Flores 2002) or they largely underestimate the objective chances of a hazard (Ludy and Kondolf 2012). Given these empirical examples it seems that people can act negligent towards risk in some cases. Yet, it is possible that there are rational reasons behind this risk negligence behaviour. Namely, perhaps the costs of flooding are subject to severe discounting of expected future losses. Particularly, when long term decisions are involved, the short term costs and benefits of a purchase decision may severely 
outweigh the long-term costs of a flood that may or may not occur. Irrespectively of the mechanisms behind risk negligence behaviour, one may expect to see no differences in prices between risk-prone and risk-free properties if risks are completely neglected.

Expected utility (EU): being well-grounded in economic literature, this model is based on the assumption that economic actors make fully rational decisions. According to the expected utility model, people weigh costs and benefits and their associated probabilities objectively, to come up with a decision that maximises their expected utility (Herstein and Milnor 1953). Brookshire et al. (1985) were the first one to apply it to the risk-prone housing market. They used the revealed preference method by looking at transaction data in an attempt to test the applicability of the expected utility model in low probability, high consequence situations. They claim that their outcomes of the hedonic analysis supports the EU paradigm. Subsequent hedonic analysis studies, which aimed at capturing the price of flood risks, mostly stem from the EU paradigm. Yet, they provide divergent results and at times find no evidence of a flood risk discount (Daniel et al. 2009). These studies do not measure the individual decisions that stand behind this aggregated outcome or how they may change with time. More detailed tests need to be done on how this individual choice model actually gives rise to the aggregated outcomes in terms of market responses to flood risk.

Prospect theory (PT): this model takes into account that people subjectively value probabilities and risks, and that risk framing and loss aversion play a role when choosing between risky options (Kahneman and Tversky 1979). Acknowledging that humans are boundedly rational, the PT model is grounded in more realistic assumptions on how people make decisions under risk. It is distinguished from EU in three ways. First, probabilities are not objective but weighted subjectively, as people tend to over-value small probabilities and under-value large probabilities. Second, when people weigh decisions they do not look at the absolute outcome but compare consequences of each decision relative to a reference point - their status quo. Third, losses are over-valued against gains due to loss aversion, i.e. it matters for the decision maker whether prospects are framed as losses or as gains. It is hypothesized that people will generally be risk averse because of over-valuation of the low probability of natural hazards, and because of loss aversion. Therefore, the price differential between safe and hazard-prone properties will be greater than that of the expected utility theory. Prospect theory has proved to be more accurate than expected utility theory in the prediction of individual decision making in a controlled environment (Kahneman and Tversky 1979). Whether the same applies for decision 
making in a risk prone housing market - where decisions and consequences are much more complex - has yet to be discovered. In particular, we would like to assess how different ways of risk framing by households would impact the aggregated price differential between safe and hazard-prone properties.

It is expected that the above mentioned risk perception models result in a capitalised price of flood risk that can be detected by the hedonic analysis of transactions (Brookshire et al. 1985). Yet this analysis of sales data, particularly in the case of natural hazard assessments, is largely confounded by collinearity in input variables (Adamowicz 1994; Earnhart 1998) and inefficient estimation of rare attributes (Earnhart 1998; Earnhart 200I). Collinearity in amenities and risks is one of the main and well-known confounding factors in the hedonic analysis of natural hazards (Donovan et al. 2007; Bin et al. 2008a; Bin et al. 2008b). Flood risk, particularly in coastal regions, is often spatially correlated with environmental amenities, leading to the fact that flood risk is understated in the revealed preference method (Atreya and Czajkowski 2016). A common approach to correct for collinearity between amenities and risks is to include some spatial factors in the hedonic analysis that control for neighbourhood effects accounting even for such attributes as sea view and sound (Bin et al. 2008b). However, de Koning et al. (2018) have shown that standard hedonic models do not always perform well in capturing trends in the market, especially for a limited set of transaction records that is common when a disaster hits. In particular, they do not capture the full complexity of space well enough, leading to the fact that estimates of how flood risk is capitalised in the market are accompanied by large errors. Although some recent attempts to better control for collinearity between risks and amenities, those including a property-specific inundation depth variable in the hedonic pricing model (Rambaldi et al. 2013; Atreya and Czajkowski 2016), have been proven successful in capturing the marginal implicit price of flood risk in more detail. Yet, while spatial dependence of risks and amenities is widely acknowledged, collinearity with other housing characteristics can play a similar role in the bias of flood risk estimates. There is currently no perfect way to control for this in the hedonic analysis.

$A B M$ can be instrumental in dealing with the issues mentioned above in the following ways. First, in a simulated market we can specify households' preferences for risk avoidance and neighbourhood quality and to control the extent to which households are sensitive to both amenities and risks. By this means we can quantify the bias in risk valuation that emerges in a market where amenities and risks are spatially correlated. Second, we can eliminate collinearity in attributes by creating a synthetic landscape with randomly allocated housing attributes, which implies no collinearities 
between risks and amenities or other factors contributing to spatial neighbourhood quality. By comparing the market outcomes between a synthetic and the real landscape where collinearities are present we can estimate the pure market impact of individual risky decision models. Additionally, we can simulate scenarios on the basis of hypothetical risk choices by individuals, and explore the consequences of changes in the risk exposure. When it comes to predicting more extreme scenarios of flood risk - especially relevant under climate change - revealed preferences methods have the drawback that they are confined to current situations. Being based on actual sales, the data is also confined to current levels of flood risk. This requires a more mechanistic approach to explore how the market will respond when the risk of flood increases under climate change (Webster et al. 2005; Stern 2008; Pryce et al. $20 \mathrm{II}$ ), something that revealed preferences models are not strong at (Adamowicz et al. 1994; Hensher et al. 1998). Stated preferences methods can be applied to address unobserved variation in attributes. Its hypothetical nature can be used to explore potential behavioural outcomes in scenarios that have not occurred.

ABMs are particularly useful to facilitate cost-benefit analyses in risk-prone areas, applying the strength of both stated and revealed preference approaches. It can be used to identify the contribution of risk exposure to housing decisions, as well as to explore the aggregate consequences of individual risk decisions and preferences for other housing attributes. While stated and revealed preferences methods are often used to assess preferences and marginal utility for specific attributes, our intention is to quantify aggregated market responses in interactions of preferences for risk and amenities. This methodology has a potential to aid scientists and policy makers with more accurate estimates of the price of natural hazards, which facilitates CBA's of risk mitigation policies.

\subsection{Data and methods}

The agent-based model used in this study is a complete model of the property market in Greenville, NC, which experiences regular floods. An extensive dataset of both GIS and housing transaction data is employed (Bin and Landry 20I3) in addition to income and housing budget data from the US (Quigley and Raphael 2004; Statista 2016; USCB 2016). Our current model is based on the RHEA model, the background and the detailed model description for which can be found in Filatova (20I5). The current version makes significant improvements on the price expectations procedure (de Koning et al. 2018) and the structure of the utility as explained further in this section. The model simulates household decisions in the flood-prone housing market. 
In particular, we model the following: I) households deciding on whether or not to sell their property, 2) a realtor agent advising sellers on their ask price, 3) buyers looking for properties within their budget, 4) buyers comparing properties based on their utility given a particular risk model and deciding to bid on a property, 5) sellers entering negotiations with buyers that offer the highest bid and 6) buyers and sellers negotiating over prices. Flood risk enters the calculations in steps 2 and 4 . In step 2 the capitalised discount for properties at risk of flood is calculated and in step 4 individual risk perception enters the utility calculation. The utility calculation is based on buyers' housing budgets, preferences for neighbourhood quality, preferences for housing characteristics and subjective risk perceptions. All of which, except for subjective risk perceptions, are heterogeneous among agents. The capitalised discount for flood risk is calculated through a hedonic analysis of sales during the simulations. Each time step the realtor agent updates his price expectations through hedonic analysis and spatial interpolation (kriging) of completed sales in the previous time step.

The initial landscape in the model is setup by uploading the entire GIS map of actual properties $(\mathrm{N}=9,793)$ and their characteristics. The expected sales price for all these properties is determined through hedonic analysis and spatial interpolation (kriging) of actual sales. For this we use the dataset of Bin and Landry (2013) for the sales in Greenville between 1992 and 2002 (N= 1,954). Two major flood events happened during this period: hurricane Fran in September 1996 and hurricane Floyd in September 1999.

In step 2, buyers randomly choose five properties within $80-100 \%$ of their housing budget, which varies among households, ${ }^{7}$ and compare the utility they gain from the properties based on their characteristics. Price negotiations and the market clearing process are modelled explicitly as explained in details in Filatova (2015). The minimum price of a property is $80 \%$ of the buyer's housing budget because properties that are cheaper will likely give a much lower utility. The buyer will not consider these properties because he can afford to spend more on housing to increase his utility. Naturally, the buyer cannot spend more than 100\% of his housing budget. Within this budget range, the buyer randomly selects five properties to compare.

\footnotetext{
7 The housing budget is derived from Quigley and Raphael (2004), Statista (2016) and the US Census Bureau (2016). With housing budget we mean the amount of money that households decide to spend on housing annually. We also take into account the relative share of income spent on housing as a function of income, i.e. we acknowledge that low income households spend a larger share of their income on housing.
} 
We did an extensive analysis to come up with the number of properties buyers consider in the utility comparison. It is an optimisation between two trade-offs: I) a large number of properties makes sure that only the properties that yield the highest utility in their price range will receive bids, and 2) a small number increases heterogeneity among buyers and makes sure that not all buyers bid on the same property. Buyers' valuation of flood-prone versus safe properties in this comparison depends on the behavioural model of risk perception used in each scenario. We analyse three different models of risk perception derived from empirical research: expected utility, prospect theory and risk negligence. They differ in the way people perceive and weigh risks when searching for a property within their budget constraints. The exact equations of each model are provided below.

\subsection{Risk Negligence}

The utility for a property without flood risk under consideration is solely based on a hedonic analysis of actual sales ${ }^{8}$, which include only the key housing characteristics that drive the variation in sales prices ${ }^{9}$. An ANOVA with the input variables described in Table 3.I yields the fraction of variance explained by each input variable, including the residual variance. This leaves us with a relative importance of each input variable in the variation of property prices, which serves as a benchmark for the buyers' preferences. The buyers' utility function can be written as:

$U_{0 L}=A_{i} * X_{i, n o r m}$

$\mathrm{X}_{\mathrm{i}, \text { norm }}$ is a vector that represents the key housing characteristics that are used as an input variable for the utility calculations, and $A_{i}$ is a weight vector that represents the relative importance of each characteristic (Table 3.I). The weights of $A_{i}$ sum up to 100 , which means that $100 \%$ of the variation in sales prices is taken into account in the buyer's utility function. The input variables, $X_{i}$, for the utility function are normalised between 0 and I depending on the sign of the hedonic regression

\footnotetext{
${ }^{8}$ We use the sales data of Bin and Landry (20I3) described earlier in this paper. We include all I 954 sales in Greenville between 1992 and 2002.

${ }^{9}$ Note that we do not include the dummy variable that describes whether or not a property is located in a flood zone. We do this for two reasons: I) it is not relevant for the risk negligence model as flood risk is not even considered, and 2) the utility function (Eq. I) is used in the other models where flood risk is included in an additional function.
} 
coefficients $^{10}$. We assume that neighbourhood quality is represented by the residuals of the hedonic regression, as all spatial factors of the hedonic analysis are replaced by spatial interpolation (kriging) of the residuals (Dubin 1992; De Koning et al. 2018) The quality of a neighbourhood may be influenced by factors such as proximity to central business district, environmental or urban amenities, quality of schools, recreational opportunities, etc.

Table 3.I. Input variables of the utility function

\begin{tabular}{ll}
\hline Input variable $\left(\mathbf{X}_{\mathbf{i}}\right)$ & Weight $\left(\mathbf{A}_{\mathbf{i}}\right)$ \\
\hline Age & 28.9 \\
Square footage & 53.8 \\
Acreage & 0.1 \\
Number of bedrooms & 0.3 \\
Gamma $*$ Neighbourhood quality & {$[0-2] * 16.9$} \\
\hline
\end{tabular}

Gamma represents the buyer's preference for neighbourhood quality. The mean value of gamma is specified between 0 and 2 , with some individual variation $(\sigma=0.05)$ between agents. We run the simulations with 5 different values of gamma $(0,0.5, I$, 1.5 and 2) to see how preferences for neighbourhood quality impacts the revealed preferences for hazard-prone properties.

\subsubsection{Expected Utility}

The utility of a hazard-prone property in EU theory is based on Eq. 3.I, with inclusion of an expected damage function. According to Kousky and Michel-Kerjan (2015) the average damage claimed from flood insurances after a flood is $25 \%$ of the property value. This serves as a benchmark for expected average losses. In other words, average of insurance claims serves as a proxy for the extent of actual damages an average household may experience during a hazard event. Thus, disutility of losses will then be:

$U_{L}=-0.25 * U_{0 L}$

With $U_{0 L}$ representing the utility of the property (Eq. 3.I). The expected damage is not just a function of expected losses in case of a flood, since it should include the

\footnotetext{
10 If the regression coefficient is positive, then the maximum gets assigned the value $\mathrm{I}$ and the minimum gets assigned the value 0 , and vice versa for negative coefficients.
} 
probability of a flood occurring. This leaves us with a temporal issue: though flood risk and flood zone are usually described as either an annual probability or a frequency of reoccurrence (100-year and 500-year flood zones), the actual probability of a flood depends on the length of residence. The probability of a flood (or $\mathrm{N}$ floods) during the length of residence is in fact a binomial distribution. This distribution is a function of length of residence (YR), number of occurrences $(N)$ and annual flood probability $(P)$. The expected length of residence is a function of turnover rate in our model: (Fraction of properties sold per year) ${ }^{-1}$, with default $\mathrm{YR}=10$. The probability of $\mathrm{N}$ floods is written as:

$P_{N}=p^{N} *(1-p)^{Y R-N} *\left(\begin{array}{c}Y R \\ N\end{array}\right)$

The annual flood probability ' $\mathrm{p}$ ' differs for each property and depends on whether the property is located inside or outside the flood zone. We can sum up the expected damage and their probabilities for a number of floods $(\mathrm{N}=0, \mathrm{I}, 2,3, \ldots)$. For our convenience we exclude the probability of more than three 100-year floods, as this seems irrelevant because of its low probability $\left(\mathrm{P}_{\mathrm{N}>3}<0.0000 \mathrm{I}\right){ }^{\prime \prime}$. The utility of a property in the expected utility model is written as:

UTILITY $=\sum_{N=0} U_{N}$ losses $* P_{N}$

And

$U_{N \text { losses }}=U_{0 L} *(1-0.25 N)$

Where $U_{N \text { losses }}$ is the utility gain for a property given a specific number of floods. In this paper discounting is not a part of the household housing purchase decision. The influence of intertemporal weighting of costs and benefits of buying a house, where flood costs may occur in various points in time and thus have different input into individual utility depending on the discounting rate, is subject for future work.

\subsubsection{Prospect Theory}

The prospect theory model is slightly more complex as it accounts for subjective weighing of probabilities, loss-aversion and framing of the utility change relative to a reference point. Unfortunately there are very few empirical examples from the real world on which to base this reference point in the housing market (but see: Bokhari and Geltner 20 I I). Which challenges the application of prospect theory in economic

II $P_{N>3}=\sum_{N=4} p^{N} *(1-p)^{Y R-N} *\left(\begin{array}{c}Y R \\ N\end{array}\right)$ is smaller than $10^{-5}$ for $\mathrm{YR}=10$. And thus we decide to neglect the probability of more than 3 floods. 
models of risky decision making (Barberis 2013). We explore four different utility reference points in order to capture a range of possible outcomes: PTnull, PT0, PTI and PT3. In PTnull the utility change of each flood scenario ${ }^{12}$ is the sum of two reference points: I) utility before owning the property, and 2) utility from owning the property in absence of floods. The total utility of a property is the sum of weighted probabilities of each scenario multiplied with the associated utility. PTnull closely resembles the expected utility model, with inclusion of the loss aversion parameter $\lambda$ and subjective weighting of probabilities $\Omega\left(P_{N}\right)$. Equations 3.6-3.8 belong to PTnull:

UTILITY $_{\text {PTnull }}=\sum_{N=0} U_{N \text { losses }} * \Omega\left(P_{N}\right)$

And

$U_{N \text { losses }}=U_{0 L} *(1-0.25 N * \lambda)$

in $\Omega\left(P_{N}\right): \gamma\left\{\begin{array}{l}0.61 \text { if } U_{N} \text { losses } \geq 0 \\ 0.69 \text { if } U_{N} \text { losses }<0\end{array}\right.$

The loss aversion parameter $\lambda$ is constant and set at 2.25 (Tversky and Kahneman 1992; Laciana et al. 2006). The non-linear function of weighted probabilities $\Omega\left(P_{N}\right)$ corresponds with Eq. 3.10 in Laciana et al. (2006). We use the values of parameter $\gamma$ found by Tversky and Kahnemann (1992): $\gamma=0.6 \mathrm{I}$ for gains and $\gamma=0.69$ for losses as these values are also found in other empirical studies.

In PTO the utility of no floods is taken as a reference point. All the other outcomes are considered as a loss of utility. In comparison to PTnull, PT0 treats a case of "no floods' as a reference point while the former considers 'no floods' as a scenario with an associated probability. Equations 3.9-3.II belong to PTO:

$\operatorname{UTILITY}_{P T 0}=U_{0 L}-\sum_{N=1} U_{N \text { losses }} * \Omega\left(P_{N}\right)$

And

$U_{N \text { losses }}=N * 0.25 * U_{0 L} * \lambda$

in $\Omega\left(P_{N}\right): \gamma=0.69$

\footnotetext{
12 A flood scenario is a hypothetical case in which a particular number $(N=0,1,2,3, \ldots)$ of floods would strike during the length residence. Each scenario has its associated probability and utility change.
} 
The reference point of PTI is one flood, i.e. the losses of one flood are already accounted for. The scenario where no flood occurs is valued as a utility gain, whereas the scenarios with 2 or more floods are valued as a utility loss. Equations 3.5 and 3.12-3.14 belong to PTI:

$\operatorname{UTILITY}_{P T 1}=\sum_{N=0}\left(U_{N}\right.$ losses $\left.-U_{1 L}\right) * \Omega\left(P_{N}\right) * \lambda$

And

$\lambda=\left\{\begin{array}{l}1 \quad \text { if }\left(U_{N \text { losses }}-U_{1 L}\right) \geq 0 \\ 2.25 \text { if }\left(U_{N \text { losses }}-U_{1 L}\right)<0\end{array}\right.$

in $\Omega\left(P_{N}\right): \gamma=\left\{\begin{array}{l}0.61 \text { if }\left(U_{N} \text { losses }-U_{1 L}\right) \geq 0 \\ 0.69 \text { if }\left(U_{N} \text { losses }-U_{1 L}\right)<0\end{array}\right.$

$U_{\mathrm{IL}}$ in Eq. 3.12 is calculated by applying Eq. 3.5 for $\mathrm{N}=\mathrm{I}$.

The reference point of PT3 is three floods. I.e. the losses of 3 floods are accounted for. All other scenarios with fewer floods are valued as a utility gain. Equations 3.5, 3.15 and 3.16 belong to PT3:

$\operatorname{UTILITY}_{P T 3}=\sum_{N=0}\left(U_{N \text { losses }}-U_{3 L}\right) * \Omega\left(P_{N}\right)$

And

in $\Omega\left(P_{N}\right): \gamma=0.61$

$U_{3 L}$ in Eq. 3.15 is found by applying Eq. 3.5 for $N=3$.

\subsubsection{Risks and amenities}

We intend to capture and quantify the effect of multicollinearity between risks and amenities, for which we apply two different approaches. One is to systematically change the mean value of gamma, the parameter that specifies buyers' preferences for neighbourhood quality (Table 3.I). As mentioned earlier, we choose five values of gamma: $0,0.5$, I, I.5 and 2. Whereby gamma $=0$ means that buyers completely disregard the neighbourhood quality, and gamma $=2$ means that buyers weigh the neighbourhood quality twice as high as expected from the analysis of transactions. In addition to changing the mean value of gamma, we include some heterogeneity in households' preferences by drawing individual gamma values from a normal distribution with $\sigma=0.05$. If there is any spatial dependence between neighbourhood quality and flood risk, then the revealed preference for flood risk will respond to changes in preferences for the neighbourhood quality. 
Another strategy that we use to disentangle the impact of flood from the influence of neighbourhood quality on flood risk capitalisation on property prices is to randomise the housing characteristics in the synthetic GIS landscape in the model. All housing characteristics, including flood risk, are randomly assigned to new properties so that there is no correlation between any of the input variables. The only variables that we maintain are neighbourhood quality and location. This approach should result in a clean effect of risk perception - revealed through hedonic analysis of transactions - that is constant and independent of gamma. We compare the outcomes of the two approaches by looking at the coefficient of flood risk in the hedonic analysis of simulated transactions.

During the simulations transactions are recorded and analysed by the realtor agent in the model so that the preferences of buyers can be captured and fed back into the formation of sellers' ask prices. The process of tracing buyers' preferences and risk perceptions by the realtor consists of 3 stages. Firstly, hedonic regression and kriging of recent sales updates the coefficients that determine the marginal implicit price of the property characteristics, including the flood zone dummy, which forms the base of the price expectation. Secondly, the price expectation is adjusted according to the number of buyers that properties with similar characteristics receive, including the risk of flood. Thirdly, sellers that are on the market for a long time will lower their ask price. The ask price formation is thus sensitive to shifts in sales prices, captured by the hedonic analysis and kriging, as well as to shifts in demand.

We run a full factorial design with 50 simulations for each combination of risk perception model (risk negligence, expected utility, PTnull, PT0, PTI and PT3), gamma $(0,0.5, \mathrm{I}, \mathrm{I} .5$ and 2$)$ and landscape (real vs random) resulting in 3000 Monte Carlo model runs. A simulation consists of 50 semi-annual time steps over a period of 25 years with no hazard event occurring. We compare the price discount revealed through hedonic analysis of sales in the simulations. This price discount is represented by the flood zone coefficient, which is the marginal implicit price for properties inside the 100-year flood zone. The unit of the flood zone coefficient roughly equivalent to the percentage price difference between properties inside and outside the flood zone. We only include sales after an equilibrium has been established.

\subsection{Results}

This section is divided into four different subsections. Each with its own experimental setup to systematically tackle the two research goals: (I) to quantitatively explore 
the market-level consequences of various behavioural models' assumptions regarding risky choices, (2) and the effect of the market-level bias that individual preferences for neighbourhood quality imply. We refer to Appendix 3.a for more details on which research objective is addressed in each section, with which settings and parameters and why.

We limit our analysis to the last 5 years of the simulations - when the simulated market has converged to an equilibrium - as we are only interested in comparing the equilibrium values. An analysis of the time series of some parameters in the model reveals that an equilibrium is generally reached after 40 time steps. All simulations that do not reach an equilibrium after 40 time steps are omitted from the analysis. For more details, see Appendix 3.b.

Table 3.2. Summary statistics for the six models of risk perception. Buyer agents have normal preference for neighbourhood quality: gamma $=1$

\begin{tabular}{lllll}
\hline Risk perception model & \multicolumn{2}{l}{$\begin{array}{l}\text { Flood zone coefficient } \\
\text { hedonic analysis }\end{array}$} & \multicolumn{2}{l}{$\begin{array}{l}\text { Number of floodplain } \\
\text { properties sold in last 5 years }\end{array}$} \\
\cline { 2 - 5 } & Mean & SD & mean & SD \\
\hline Risk negligence & 0.043 & 0.021 & 251 & 20.8 \\
Expected utility & -0.001 & 0.022 & 246 & 19.4 \\
Prospect theory no flood & -0.186 & 0.034 & 230 & 26.0 \\
Prospect theory I flood & -0.803 & 0.111 & 181 & 24.7 \\
Prospect theory 3 floods & -0.250 & 0.037 & 223 & 23.0 \\
Prospect theory null & -0.369 & 0.047 & 212 & 24.0 \\
\hline
\end{tabular}

\subsection{Revealed preferences for risk in the real landscape: prospect theory vs expected utility}

In this section, we attempt to tackle the first research goal of quantifying the marketlevel impact of different risk perception models. We perform a hedonic analysis of simulated sales in the actual landscape and buyers that have no bias in preference for neighbourhood quality (gamma=I). Table 3.2 shows the mean discount for floodprone properties for each risk perception model. Risk negligence results in a positive revealed preference for risk-prone properties, and the expected utility model approximately shows indifference between risk-prone and safe properties. Both models show that the revealed preference method underestimates buyers' risk perceptions. In other words, there is no clean effect of different risk perception models on the marginal implicit price paid for risk avoidance. Furthermore, prospect theory shows a great variance in flood zone coefficient (mean values range between 
-.186 and -.803) depending on the chosen reference point. It implies that the marginal implicit price of flood risk in this model strongly depends on framing. PTnull and PTI have a much higher standard deviation of the flood zone coefficient and appear unrealistic in comparison to the empirically estimated flood risk discount in this area. Therefore, PTnull and PTI are omitted from further analysis.

It should be noted that the large discount in PTI is mainly caused by loss aversion in case more floods occur and by the fact that people under-weigh the probability of no flood. In PT3, people already account for 3 floods and so there is no loss aversion. Similarly, the coefficient for PT0 is closer to zero than PT3. The primary reason is that in PT3, people under-weigh the probability that no flood occurs, making them undervalue the expected utility gain from buying a flood-prone property in this scenario.

Table 3.3. Summary statistics for the six models of risk perception. Buyer agents have no preference for neighbourhood quality: gamma $=0$

\begin{tabular}{|c|c|c|c|c|c|c|}
\hline \multirow[t]{2}{*}{ Risk perception model } & \multicolumn{3}{|c|}{$\begin{array}{l}\text { Flood zone coefficient } \\
\text { hedonic analysis }\end{array}$} & \multicolumn{3}{|c|}{$\begin{array}{l}\text { Number of floodplain } \\
\text { properties sold in last } 5 \text { years }\end{array}$} \\
\hline & mean & SD & $\begin{array}{l}\text { Difference } \\
\text { with } \\
\text { gamma = I }\end{array}$ & mean & SD & $\begin{array}{l}\text { Difference } \\
\text { with } \\
\text { gamma = I }\end{array}$ \\
\hline Risk negligence & 0.000 & 0.018 & -0.043 & 242 & 23.0 & -9 \\
\hline Expected utility & -0.037 & 0.026 & -0.036 & 232 & 25.6 & -14 \\
\hline Prospect theory no flood & -0.212 & 0.050 & -0.026 & 236 & 24.2 & +5 \\
\hline Prospect theory 3 floods & -0.287 & 0.052 & -0.037 & 242 & 19.6 & +19 \\
\hline
\end{tabular}

\subsubsection{Restraining the preferences for neighbourhood quality}

Next, we address the market-level bias driven by individual preferences for neighbourhood quality. We compare the statistics of Table 3.2 with the simulations where buyers are insensitive to neighbourhood quality: gamma $=0$. This comparison should reveal any spatial dependence of flood risk and neighbourhood quality. Given the previous results, it is expected that individual risk perceptions are better captured by the hedonic analysis, resulting in a larger price discount for flood risk (Table 3.3). Indeed, we see that the flood zone coefficient in the hedonic analysis is consistently lower than in the simulations with gamma=I. The marginal effect of neighbourhood quality on risk perceptions in the revealed preferences method ranges between -.043 and -.026 . Suggesting that the marginal implicit price of flood 
risk in this particular case may be understated by $2.6 \%$ to $4.2 \%$ of the property value, which is more than one standard deviation of the flood zone coefficient.

\subsubsection{Excluding the effect of neighbourhood amenities: revealed preferences for risk in the random landscape}

In the previous section we have looked at how preferences for neighbourhood quality may cause a systematic bias in the revealed preferences for flood risk. Alternatively, we can eradicate spatial dependency between neighbourhood quality and flood risk by randomly assigning characteristics to properties in the landscape. This is an alternative way to correct for preferences in the neighbourhood quality, and at the same time correct for collinearity with any other housing characteristics so that the clean impact of micro-level risk perceptions can be measured on a macro scale. Thereby addressing both research objectives. We see that the flood zone coefficient in the hedonic analysis ranges between 0 in the risk negligence model to -0.35 in the PT3 model (Table 3.4). The latter indicates a price differential of $-30 \%$ between risk-prone and safe properties.

Furthermore, the comparison of this randomised landscape with the real landscape reveals an even greater bias in the flood zone coefficient estimate. The bias in marginal implicit price of flood risk ranges between $4.2 \%$ and $9.7 \%$.

Table 3.4. Summary statistics for the six models of risk perception in the randomised landscape. Buyer agents have normal preference for neighbourhood quality: gamma = I

\begin{tabular}{lllllll}
\hline Risk perception model & \multicolumn{2}{l}{$\begin{array}{l}\text { Flood zone coefficient } \\
\text { hedonic analysis }\end{array}$} & \multicolumn{2}{l}{$\begin{array}{l}\text { Number of floodplain } \\
\text { properties sold in last } 5 \text { years }\end{array}$} \\
\cline { 2 - 7 } & mean & SD & $\begin{array}{l}\text { Difference } \\
\text { with real } \\
\text { landscape }\end{array}$ & mean & SD & $\begin{array}{l}\text { Difference } \\
\text { with real } \\
\text { landscape }\end{array}$ \\
\hline Risk negligence & 0.000 & 0.013 & -0.043 & 269 & 18.0 & +18 \\
Expected utility & -0.049 & 0.015 & -0.048 & 263 & 15.8 & +17 \\
Prospect theory no flood & -0.268 & 0.025 & -0.082 & 271 & 18.7 & +41 \\
Prospect theory 3 floods & -0.352 & 0.071 & -0.102 & 264 & 16.6 & +41 \\
\hline
\end{tabular}

\subsubsection{Disentangling market impacts of preferences for risk from neighbourhood quality preferences}

From the previous we can conclude that preference for neighbourhood quality (and perhaps also other housing characteristics) has a clear effect on the estimate of flood risk. Next, we assess whether this effect is consistent for different values of gamma, 
and if the effect increases when agents have a strong preference for neighbourhood quality in their utility calculations (Fig. 3.I). All risk perception models consistently reveal that an increased preference for neighbourhood quality diminishes the flood discount in the hedonic analysis of sales (values approach to zero or increase in the positive range).

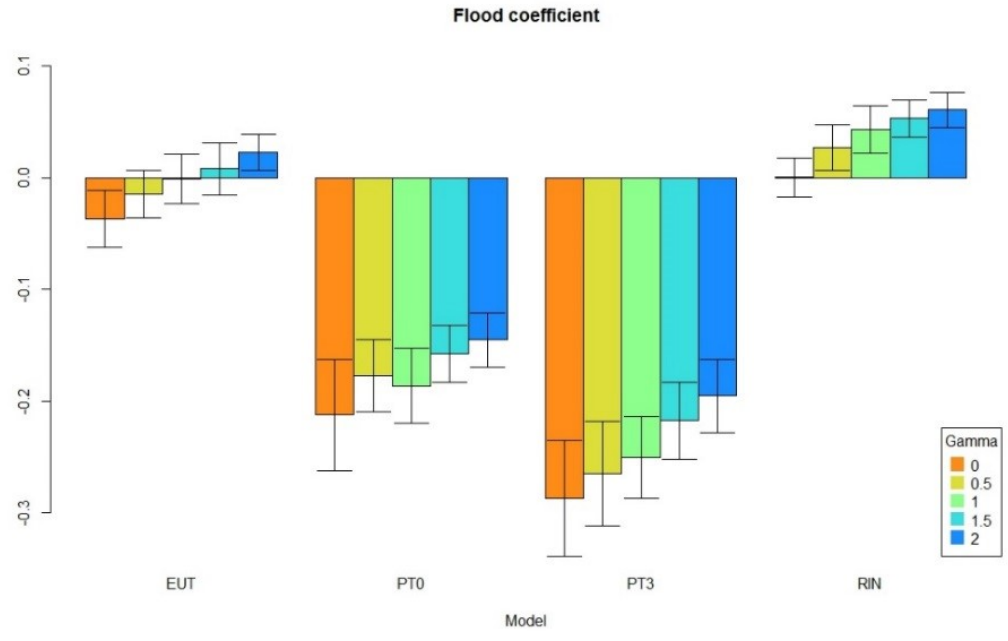

Figure 3.I. Discount for flood-prone properties in the last 5 years of the simulations in the real landscape. The models shown are expected utility, risk negligence, prospect theory with no floods as reference point and prospect theory with 3 floods as reference point. Gamma indicates buyer's preference for neighbourhood quality. Error bars indicate one standard deviation, i.e. effect size.

This effect is likely caused by spatial correlation between risks and amenities in the landscape. When we remove this correlation by randomly assigning new housing attributes to properties we see that the correlation between gamma and the flood zone coefficient disappears (Fig. 3.2). In this case, the discount for flood-prone properties is constant over a range of preferences for neighbourhood quality, which means that in this landscape, preferences for neighbourhood quality and risk preferences can be valued separately. Furthermore, we see that the discount for flood-prone properties is much closer to the values that can be expected from observing the individual risk-preferences of households. 


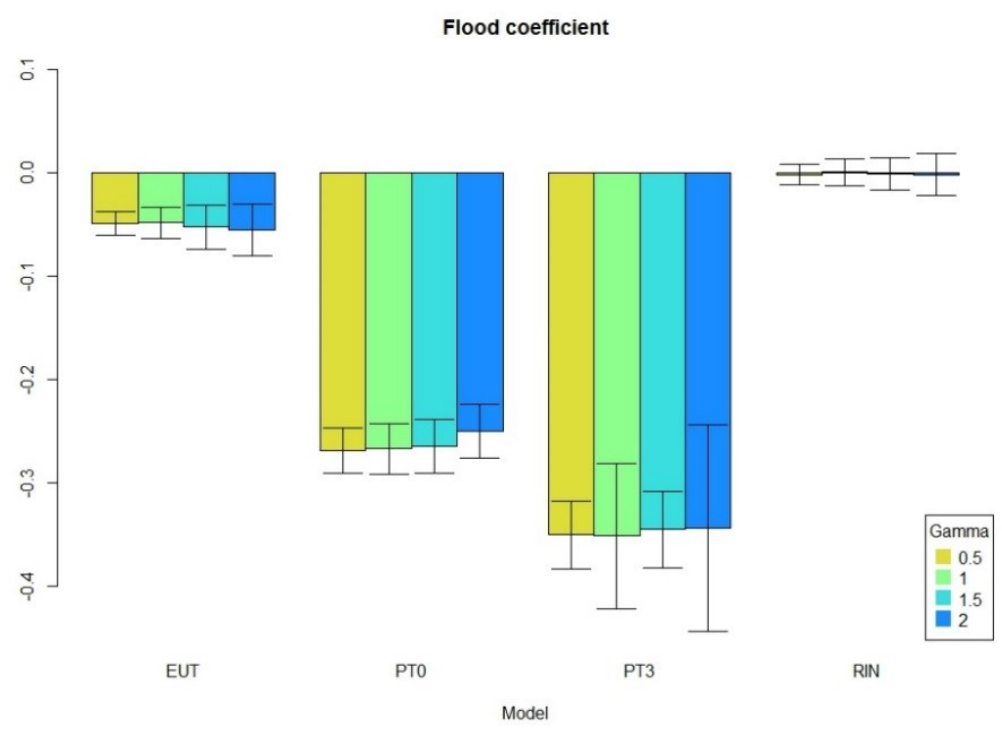

Figure 3.2. Discount for flood-prone properties in the last 5 years of the simulations in the random landscape. The models shown are expected utility, risk negligence, prospect theory with no floods as reference point and prospect theory with 3 floods as reference point. Gamma indicates buyer's preference for neighbourhood quality. Error bars indicate one standard deviation, i.e. effect size.

\subsection{Discussion}

The aim of this paper is to quantitatively explore (I) the market-level consequences of various behavioural models' assumptions regarding risky choices at the individual level, and (2) the effect of the market-level bias that individual preferences for neighbourhood quality imply.

Regarding the first goal, our results demonstrate that the marginal implicit price of flood risk is largely affected by individual perceptions of risk and loss aversion. The 'clean effect' of individual risk perceptions on the flood discount for hazard-prone properties in the simulations, where economic agents are indifferent about neighbourhood quality, ranges between $0 \%$ in the risk negligence model to $-25 \%$ in the PT3 model on average. The bias in revealed risk valuation due to the preference for neighbourhood quality ranges between $+2.6 \%$ and $+4.2 \%$, an effect more than one standard deviation. Empirical studies mostly found a discount for hazard-prone properties without hazard events at the upper end of this range: $-2 \%$ on average, although the values vary between $-52 \%$ and $+58 \%$ (Daniel et al. 2009). The risk 
negligence model yields a flood discount value that closely resembles these empirical estimations, followed by EU and PT, while EU is closer to the empirical values when preferences for neighbourhood quality are included. This is an interesting finding since PT is argued to be superior in explaining individual risky choices, and we expected that a property market with PT traders will resemble the actual market in hazard-prone areas more accurately than for example EU. There might be several explanations for the seemingly poor performance of the PT-traders market. Firstly, PT envisions that people overestimate low probability high consequence events. Thus, the result that markets with PT agents overestimate flood risk discount is in fact a natural outcome. However, while PT may be superior in explaining individual decisions under risk, it may not be the case on a market level. Namely, while there are individual biases including loss aversion and subjective weighting of probabilities, there might be some 'wisdom of crowd' on a market level when individual irrationalities may well be sorted out and priced out when many agents interact. Perhaps it is a 'law of large numbers' at work on the aggregated market level. Secondly, the EU model does not produce a perfect fit for the empirically estimated flood risk discount. Thus, while some PT factors may be still at work, there may be something else not included in our simulation model or in the data we use. Our data is still quite aggregated, which we try to disaggregate when initializing the synthetic trader population based on income intervals, average housing budget share and average demand curve. Most importantly, we do not take into account the full heterogeneity in preferences that could be present on an individual level. When we assume that all agents are equally biased in their risk behaviour, we amplify the differences in behavioural risk models on an aggregate scale. Our results do not give a conclusive answer to which behavioural model of decisions under risk is the most accurate. It is an interesting question for future research given the availability of disaggregated data.

Importantly, our results show that different assumptions in risk perception models lead to fundamentally different market level outcomes in terms of capitalised risk discounts. We quantify the marginal effect of risk on property prices under various behavioural models scenarios. The large variance in flood discount between them indicate that risk perception and potentially loss aversion of individual households can have a strong impact on property prices in hazard-prone areas (McCluskey and Rausser 200I), which challenges cost-benefit analyses in risk mitigation policies. It demands a clear understanding of the (heterogeneity in) individual choices of households in risk-prone property markets. This individual approach, in combination with our agent-based model, can aid policy makers to respond both timely and adequately to changes in households' risk behaviours. 
With respect to the second objective, to disentangle the biases that housing attributes such as neighbourhood quality may impose on the pure effect of capitalised risk perceptions in property prices we run our model with the landscape and housing characteristics randomised. Any sample of sales for the hedonic analysis is then always clean in terms of multicollinearity in the input variables. A mismatch in risk valuation between stated and revealed preference methods can easily be caused by this confounding mechanism (Adamowicz et al. 1994; Timmermans et al. 1994). Only in a hypothetical landscape with no correlation in housing characteristics and space can we obtain a direct relation between individual level preferences (e.g. risk perceptions) and revealed risk preferences. We found that the real landscape - that contains all the limitations of empirical hedonic studies such as multicollinearity in input variables - causes systematic biases in the valuation of flood risk through revealed preferences. These biases are likely caused by: I) preferences for neighbourhood quality, which is often spatially correlated with risks, and 2) multicollinearity of risk and other housing characteristics. Correlation between amenities and risks in particular is for a large part responsible for the bias in risk valuation through hedonic analysis. Although this is widely acknowledged in hedonic literature (Donovan et al. 2007; Bin et al. 2008b), there is no perfect solution to disentangle the two and to quantify the pure value of risk. While some recent studies have captured more detailed location-specific information on flood risk in their hedonic pricing models, which allows for predicting the property specific price of flood risk in more detail than our model (Rambaldi et al. 2013; Atreya and Czajkowski 2016). Our ABM allows us to quantify the marginal prices for risks and amenities separately. This gives us more insight in the discrepancy between individual and market level risk valuations. The capitalised value of flood risk at the market level is consistently lower in the synthetic landscape (4.2\%-9.7\% lower on average) where amenities and risks are randomized, irrespectively of the behavioural risk model used at the individual level. Notably, the capitalised value of risk, which is positive under the high preferences for neighbourhood quality scenarios in EU and risk negligence models, switches signs. Since hedonic analysis literature sometimes finds positive flood risk discount, our approach offers a virtual laboratory for such a quantitative test. It can be applied to a wide range of cases and enhances the performance of revealed preference methods by controlling for confounding factors more precisely.

In conclusion, we found some clear differences in the marginal implicit price of flood risk among different behavioural risk perception models. At the same time we quantitatively assessed that spatial correlation between risks and amenities and the preference for neighbourhood quality can have strong confounding effects on the valuation of flood risk through the revealed preferences method. Simulations, such 
as our agent-based housing market, can be instrumental in supporting empirical and theoretical economic research especially when used in symbioses with the traditional techniques. The purpose is not to 'fit' the results of our ABM with an empirical dataset of actual sales. Our ABM is used to quantitatively explore the difference in individual and market-level risk valuations, given the limitations of using a real dataset. As such, it serves as a bridge between alternative assumptions on the individual household level and the data on a market level. The approach presented in this paper has some limitations that open new avenues for future research. It would be important to zoom into the choices of individual households that underlie the price of flood risk on a market-level. The data used in our model is not sufficient to give a conclusive answer to how risk governs their decisions. Behavioural theory regarding risky choices may work well in experiments with clear and simple choices. Contingent valuation methods and choice experiments provide valuable information but cannot offer a full control. Even so these hypothetical choice experiments are still far less complex than actual housing decisions, and risk is just one of the many factors that impacts the choice of housing (Timmermans et al. 1994; Collen and Hoesktra 200I; Zinas and Jusan 20I2). While in the hedonic literature many found a clear discount for risk-prone properties (Murdoch et al. 1993; De Blaeij et al. 2003), surveys suggest that people do not consider risk when purchasing a house (Burningham et al. 2008; Willis et al. 20I I). The price for flood risk that is found in empirical studies could be unrelated with conscious choices and risk perceptions of individuals. Daniel et al. (2009) even suggest that there may be a publication bias in the hedonic literature. We challenge future research to disentangle the mechanisms of how risk exposure capitalises into property prices. 



\title{
CHAPTER 4 - Capitalization of Flood Insurance and Risk Perceptions in Housing Prices: An Empirical Agent-Based Model Approach
}

This chapter is accepted for publication as a paper in the journal 'Southern Economic Journal' (16/8/2018)

\begin{abstract}
Federally regulated or insured lenders in the United States are mandated to require flood insurance on properties that are located in areas at high risk of flooding. Despite the existence of this mandatory flood insurance requirement, takeup rates for flood insurance have been low and the federal government's exposure to uninsured property losses from flooding remains substantial. Meanwhile the value of capital at risk varies significantly driven by flood events and changing risk perceptions, which necessitates mechanisms that stabilize these dynamics. In this paper we discuss how a scenario of complete insurance uptake, under various risk attitudes, affects the value of properties in the 100-year and 500-year flood zone. Our results indicate that an increase in flood insurance uptake may provide such a mechanism by lowering the value of capital at risk in the flood zone consistently, independent of homeowners' risk attitudes. We apply an empirical adaptive agentbased model to examine the capitalization of insurance costs, risk premiums and their interaction in housing prices. Our approach combines widely-used empirical hedonic analysis with the computational economic framework. We highlight the usefulness of our method in capturing the marginal implicit price of homeowners' preferences that may change over time, and assess separately the effect of various factors and policies on property values, illustrating the agent-based modeling as a valuable complement to traditional hedonic analysis.
\end{abstract}
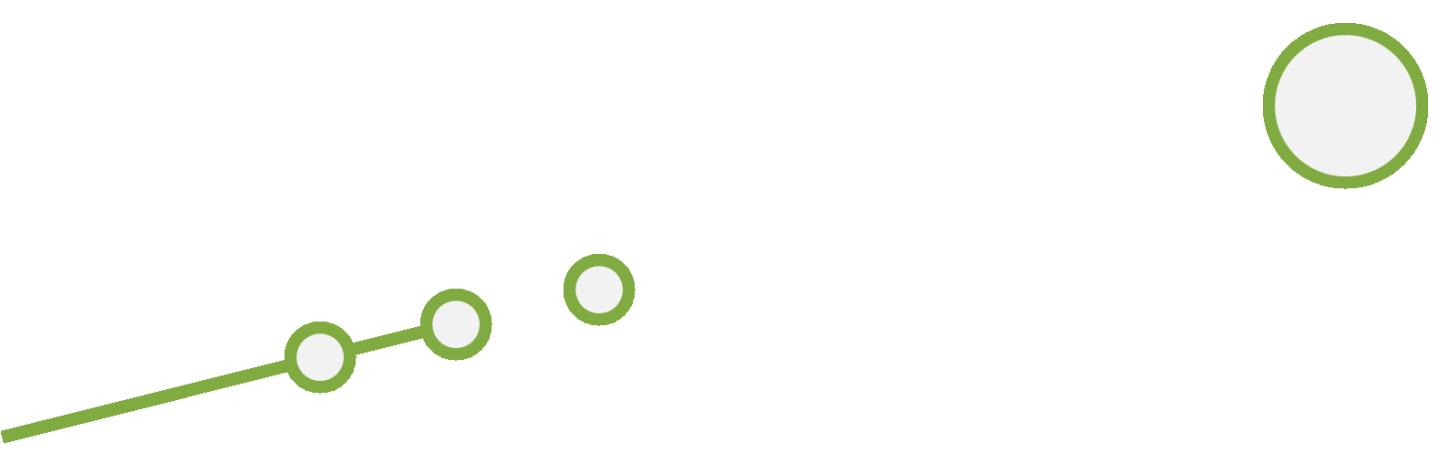



\section{4.|| Introduction}

Flooding is the most frequent and costly natural disaster in the world. In the United States, the National Flood Insurance Program (NFIP) aims to redistribute risks and provide immediate relief funds to meet the escalating costs of floods. The success of the NFIP may depend on appropriate pricing and also making the flood insurance widely available to homeowners at risk. Certain property owners in Special Flood Hazard Areas (SFHAs) in the communities that participate in the NFIP are required to purchase and retain flood insurance for the life of their mortgage loans. Yet, despite the existence of this mandatory purchase requirement, take-up rates for flood insurance have been low (Browne and Hoyt 2000; Kriesel and Landry 2004; Michel-Kerjan and Kousky 2010), reaching up to only $49 \%$ of single-family homes in the designated zones nationwide (Dixon et al. 2006). A concern on the low take-up rates has been often expressed following major flood events.

The full insurance take-up is desirable not only because it creates a safety net but also provides immediate financial funds essential for a recovery process. It is argued that insurance may also lead to a lower capital at stake due to a decrease in property prices (Frame 1998) and communicates risks to homeowners (Nyce et al. 2015). With complete information about flood risks and rational behaviors, risk-based housing price differentials should persist as long as risk differential exists. However, empirical evidence suggests the flood risk discount may diminish over time. Atreya, Ferreira, and Kriesel (2013) find that house prices fell sharply after a hundred year flood event and the discount disappeared after four years following the flood. Bin and Landry (2013) find a significant housing price discount following Hurricane Fran and Floyd, which declined steadily over five to six years to the baseline. This dependence of housing price differentials on the timing after a flood has confounded the assessments of willingness to accept for flood risks, and consequently further analyses of flood risk management options. It is an open question how flood insurance, individual risk attitudes of potential homeowners, and the resulting property prices (which form the basis for direct flood damage assessments) interact.

This paper aims to quantitatively explore how the full uptake of flood insurance and households' risk attitudes interact and affect property prices in flood zones. In the pursuit of this goal we pose two research questions: (1) How does the housing market endogenously change with an introduction of a hypothetical full insurance uptake policy that affects households' budget constraints? (2) How do risk attitudes of individual homeowners affect capitalized flood risks, in absence or presence of the full insurance uptake? Specifically, we assess whether the flood insurance has an effect on housing values, whether this effect differs across flood zones and among 
behavioral models, and how it capitalizes in housing prices after the introduction of compulsory insurance for all flood-prone properties.

To address this challenge we employ a novel combination of the traditional hedonic analysis (HA) and an agent-based computational economic model (ACE). Hedonic analysis is a common method to study how various attributes of a composite good contribute to its value (Rosen 1974). It has been actively applied to residential housing markets and often to elicit the impact of flood risks on property values (Macdonald, Murdoch, and White 1987; Bin and Polasky 2004; Kousky 2010; Atreya and Czajkowski 2016). In general, HA utilizes recorded transaction prices that represent the net results of bargaining between buyers and sellers (Bockstael 1996). However, the implicit price estimates based on past transactions may not be robust when underlying individual behaviors or market conditions change, altering traders' willingness-to-pay and willingness-to-accept. ACE approaches to modeling property markets represent a flexible alternative (Nolan et al. 2009; Parker 2014). This simulation method models economic systems with heterogeneous agents that act, interact and learn according to defined rules (Farmer and Foley, 2009). ACE markets are usually based on decentralized bilateral trading among agents (Tesfatsion and Judd 2006; Tesfatsion 2017). In other words, quantities and prices, at which goods are exchanged on market, are defined not by an intersection of aggregated market demand and supply curves but through price negotiations between a pair of agents: a buyer and a seller. Due to this flexible model structure, ACE models provide a platform for wider exploration of out-of-equilibrium dynamics (Arthur 1999), agent heterogeneity (Kirman 1992), bounded rationality (Simon 1997), and interactions and learning between agents (Axtell 2005). The major strength of using an ACE model in our study is that we can systematically run scenarios with various behavioral assumptions, such as individual risk tolerance, as well as insurance or its absence, to create a large dataset of simulated transactions suitable for traditional hedonic analysis. By analyzing this pool of data, we are able to disentangle the effects of different factors - insurance, risk attitudes, or their interactions - and to quantitatively assess their marginal contribution on the flood risk discounts capitalized in housing prices.

The strengths and limitations of agent-based economic models have been well documented (Irwin 2010; Meen 2016). Our agent-based model integrated with HA combines the strengths of two methodologies. We use ACE to trace changes in the aggregated housing market dynamics endogenously as flood insurance costs affect buyers' budget constraints, where both budget constraints and buyers' risk attitudes alter the willingness-to-pay for properties in a floodplain. Also, HA provides an 
empirical ground to elicit buyers' preferences for housing attributes and serves as a vehicle to model adaptive price expectations. When ACE and HA are combined, a bilateral housing market allows exploring a shift between simulated hedonic equilibria while directly tracing the dynamics of implicit prices of flood risk given different behavioral risk models and assumptions about the flood insurance.

The next section describes the study area and data, briefly discussing a background on the NFIP relevant to the understanding of the flood insurance requirement. The details of the method are presented in Section 3. In Section 4 we discuss in which cases the flood insurance requirement may be associated with lower property values in flood-prone areas and how risk attitudes of homebuyers may offset the overall reduction. An understanding of these mechanisms may help insurance practitioners and policy-makers make informed decisions on managing flood risks and improving resilience. We conclude with a discussion of our findings and opportunities for future work.

\subsection{Study Area and Data}

The NFIP was created in 1968 as a result of the passage of the National Flood Insurance Act which enabled property owners in participating communities to purchase insurance as a protection against flood losses in exchange for local floodplain management regulations. Concerns about the costs of flooding and low take-up rates led Congress in 1973 to make the purchase of flood insurance mandatory for property-owners in 100-year floodplains with a mortgage from federally backed lenders. Still, concern is often expressed following major flood events that many at-risk homeowners remain without coverage. An estimate of takeup rates in 100-year floodplains by RAND Corporation finds high regional variation, with the South and West having the highest take-up rates of around $60 \%$, while in the Midwest, take-up rates are only around 20-30\% (Dixon et al. 2006). As of August 2016, about 5.1 million policies were in-force nationwide (CBO 2017). The significant NFIP debt mainly associated with Hurricanes Katrina (2005) and Sandy (2012) generated broad interest in reforming the program which includes a gradual rate increase for higher-risk areas and the mandatory disclosure of flood risk. The flooding from Hurricane Matthew (2016) and Harvey, Irma, Maria, and Nate (2017) have further complicated matters by adding another over $\$ 10$ billion to the program's $\$ 25$ billion debt. 


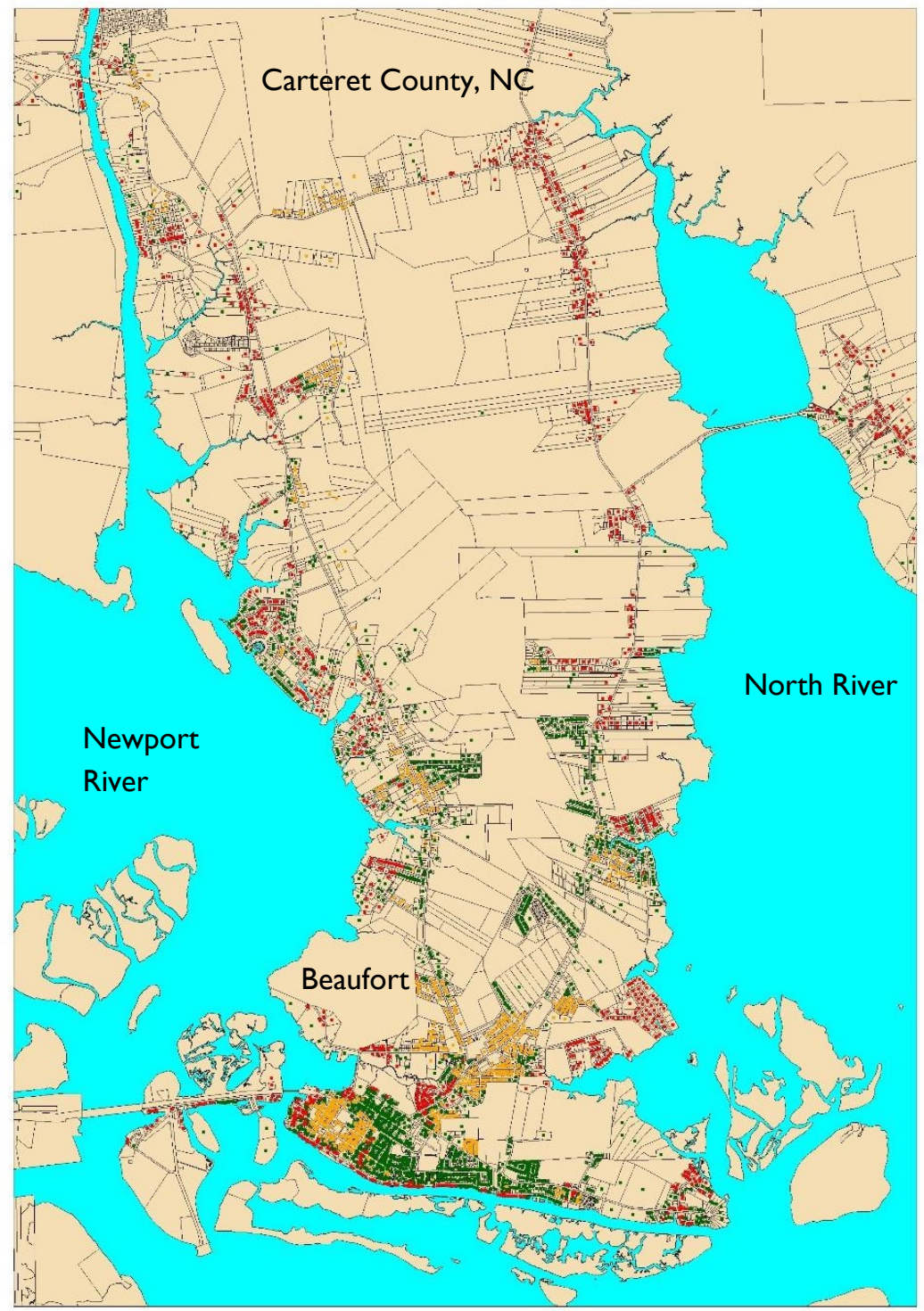

Figure 4. I. Map of the study area. Dots indicate the centroid of a parcel with colors indicating the parcels' flood zone designations: red $=100$-year, orange $=500$-year and green $=$ safe zone.

Our study area encompasses the town of Beaufort in Carteret County, North Carolina (Fig. 4.I). The area is in general low lying and prone to flooding with varying risks. Carteret County is comprised of mainland and coastal barrier islands. We exclude the barrier islands of Carteret County, which usually covers the most 
hazardous areas with wave velocity. For the model initialization, we employ spatially referenced data from multiple GIS datasets on the locations of residential housing, environmental amenities and neighborhood quality, flood zone designations, and data on structural characteristics of properties including age, structure square footage, lot size, and number of bedrooms (Table 4.I). We focus on the single family residential transactions between $200 \mathrm{I}$ and 2004, where sales prices are inflation-adjusted using a Consumer Price Index to report figures in September 2004 dollars. This hedonic analysis is used to calibrate the pricing of housing at initialization of the integrated HA-ACE model.

The digital flood maps obtained from the North Carolina Floodplain Mapping Program are used to identify properties within flood zones. Flood zone maps provide the location and extent of floodplains in the county. We denote two major categories based on the recurrence interval: a 100-year floodplain, where flood insurance is mandatory for homeowners who finance their purchase through federally regulated lenders, and a 500-year floodplain. The study area in our GIS dataset contains 7,106 parcels, and 3,48I of which are used in the analysis. Among the residential properties, $48 \%$ are located outside the flood zone, $30 \%$ and $22 \%$ are in 100 -year and 500-year floodplains, respectively.

Amenities such as the proximity to coastal water (i.e., ocean, sound, and intracoastal waterways), water frontage, and boat access are highly valued in the coastal housing market. In addition, other locational characteristics such as distance to the nearest central business district (downtown Morehead City), the nearest highway, urban amenities, quality of schools and recreational opportunities may affect housing prices. Given the spatial correlation/multicollinearity among these spatial attributes, we assume that neighborhood quality is represented by the residuals of the hedonic regression. We apply spatial interpolation (kriging) to quantify the impact of neighborhood quality on property prices (de Koning, Filatova, and Bin 2017). To endow agents with heterogeneous incomes and housing budgets based on the empirical data (Statista 2016; United States Census Bureau 2016; Quigley and Raphael 2004), the results of an ANOVA of the main housing attributes are used as proxies for households preferences for the corresponding attributes as described in the next section.

\subsection{Methods}

To examine capitalization of insurance premiums in housing prices we apply a spatial ACE model combined with hedonics analysis. We build upon other agent-based 
models of property markets (Parker and Filatova 2008; Gilbert et al. 2009; Ettema 201 I; Magliocca et al. 20I2; Magliocca, McConnell, and Walls 20I5). Our approach makes a step forward towards the empirical modeling of ACE by running a policy scenario based on HA, GIS data, and distribution of households' preferences and incomes, and the explicit treatment of different behavioral models at the agent level. The core of our model is a simulated bilateral market (Fig. 4.2) where heterogeneous household agents (buyers and sellers) exchange heterogeneous spatial goods (houses) (Filatova 2015).

The ACE model is coded using the $R$ statistical language, and is complemented by $\mathrm{HA}$ to examine the effects of the flood insurance take-up on housing prices. The initial model is setup by uploading the entire GIS map of actual properties and their attributes. At the start of the simulation the expected sales prices for properties are assigned using HA of the actual sales data combined with spatial interpolation (kriging) of the residuals (de Koning, Filatova, and Bin 2017). The model dynamics is realized through a sequence of steps: (I) households who own a property may decide to put it on a market, (2) real-estate agents advise sellers on their ask price given the current market trends, (3) buyers search for a property that gives them the highest utility under the budget constraint and place a bid, (4) sellers engage in price negotiations with the buyers who place the highest bid and (5) real-estate agents update their price prediction based on the recent realized bilateral transactions. In this paper, we further extend our experiments by altering buyers' budget constraints through insurance premiums for housing in flood zones and individual risk perception. The logic behind buyers and sellers' decisions is discussed below.

There are four scenarios considered in this study: no enforced insurance requirement and the full take-up of flood insurance ${ }^{13}$, tested under two behavioral risk models. Namely, we compare the aggregated market outcomes assuming that individual households are expected utility maximizers with risk-neutral behaviors vs. when they completely neglect flood risk when purchasing a house. We perform 50 Monte Carlo runs for each scenario while running the empirical agent-based housing market model for 100 periods assuming that each period equals to 6 months.

\footnotetext{
${ }^{13}$ We assume the full penetration of flood insurance from the start. ACE can also be instrumental to explore a process of the insurance take up (Haer et al. 2017, Dubbelboer et al. 2017).
} 


\subsection{Buyers behavior: risk avoidance and flood insurance}

The HA-ACE simulation model is initialized with thousands of heterogeneous household agents differentiated by budgets, and housing- and location preferences, which search for a house to buy. Each time step buyers randomly choose five properties affordable for their housing budget which varies across households. Insurance influences buyers' decisions first in this phase. In the model with $100 \%$ insurance uptake, buyers limit their budget for properties in the flood zone to reserve fund for insurance costs. Next, buyers choose one among five affordable properties that gives them the highest utility. Their utility for owning a property depends on a bundle of property attributes, neighborhood quality and a potential exposure to flood hazard. The base multi-attribute utility function $\left(U_{O L}\right.$, Eq.4.I) for a house in a safe area is parameterized using weights $\left(A_{i}\right)$ that reflect relative importance of each characteristic $\left(X_{i, n o r m}\right)$ :

$U_{0 L}=A_{i} * X_{i, n o r m}$

The weights $\left(A_{i j}\right)$, which indirectly measure preferences of an agent $j$ for a particular attribute $i$, are based on de Koning, Filatova, and Bin (2017) which examines HA of actual sales using only the key housing characteristics ${ }^{14}$ that drive the variation in sales prices ${ }^{15}$. Table 4.1 presents the ANOVA results illustrating the fraction of variance explained by each input variable, including the residual variance, which captures neighborhood quality. The relative importance of each input variable in the variation of property prices in Table 4.1 serves as a benchmark for the buyers' preferences. The sum of all $A_{i j}$ equals to 100 for every agent $j$. The property attributes $\left(X_{i}\right)$ are normalized between 0 and $I$ depending on the sign of the hedonic regression coefficients ${ }^{16}$.

\footnotetext{
${ }^{14}$ We used data from Bin and Landry (2013) that includes 9,793 residential property sales between 1992 and 2002.

${ }^{15}$ Note that we do not include the dummy variable that describes whether or not a property is located in a flood zone here as it enters the analysis later.

${ }^{16}$ For a positive regression coefficient the maximum gets assigned the value $I$ and the minimum gets assigned the value 0 . It is vice versa for negative coefficients.
} 
Table 4.I. Relative importance of property attributes in the agents' utility function

\begin{tabular}{llll}
\hline Input variable $\left(\mathbf{X}_{\mathbf{i}}\right)$ & Mean weight $\left(\mathbf{A}_{\mathbf{i j}}\right)$ & Min weight $\left(\mathbf{A}_{\mathbf{i j}}\right)$ & Max weight $\left(\mathbf{A}_{\mathbf{i j}}\right)$ \\
\hline Age & 18.2 & -99.2 & 100 \\
$\ln ($ Square footage) & 35.2 & -19.9 & 100 \\
$\ln ($ Acreage) & 4.4 & -49.9 & 100 \\
Number of bedrooms & 6.4 & -49.9 & 100 \\
Neighbourhood quality & 35.8 & 0.10 & 95 \\
\hline
\end{tabular}

When choosing a location within designated flood zones, a household operates under the conditions of uncertainty and may experience flood damage. Kousky and MichelKerjan (2015) estimates the average flood insurance claim after a flood as approximately $25 \%$ of the property value. This serves as a proxy for the extent of actual damages an average household may experience during a flood. We model the disutility of losses as:

$U_{L}\left\{\begin{array}{lr}=0.25 * U_{0 L} & \text { without insurance } \\ =0.10 *\left(0.25 * U_{0 L}\right) & \text { with insurance }\end{array}\right.$

with $U_{0 L}$ representing the utility of the property in the absence of flooding (Eq. 4.I). The disutility of losses in case of insurance is 10\% of that without insurance because we account for losses that are covered by the insurance. This is to take into account the disutility of losses that cannot be not covered, such as the emotional distress and deprivation of being displaced along with the loss of personal or family items with sentimental value that goes beyond the financial losses. The total expected damage is a function of expected losses in case of a flood and the probability of a flood occurring. The actual probability of a certain number of floods $(N)$ experienced by a household in 100-year and 500-year flood zones depends on the length of residence (YR). Following de Koning, Filatova, and Bin (2017), the probability of $\mathrm{N}$ floods is estimated as:

$P_{N}=p^{N} *(1-p)^{Y R-N} *\left(\frac{Y R}{N}\right)$

Whereby $p$ is the annual probability of a flood occurring, reflected by the 100 -year and 500-year flood zone. Consequently, the expected utility (EU) a household receives from owning a property equals:

$E U=\sum_{N=0} U_{\text {Nlosses }} * P_{N}$

given that

$U_{N \text { losses }}=U_{0 L}-U_{L} * N$ 
where $U_{N}$ losses is the utility gain for a property considering a specific number of floods. Eq. 4.4 is based on a simple additive Von Neumann and Morgenstern utility function (Pollak, 1967). Note that the shape of our utility function is linear with losses (Eq. 4.2), implying that buyers in our model are risk-neutral ${ }^{17}$. We acknowledge that this assumption is a simplified one, and ignores the risk-averse behaviour under a concave utility function. Yet, to parameterize the utility functions for the composite good on empirical data has already been a challenge, especially because we model heterogeneous preferences among buyers ${ }^{18}$. The degree of concavity or convexity of the utility functions would add a substantial complexity and require more empirical data to parameterize. Given that validation of agent-based models is challenging enough in itself (Windrum et al. 2007; Fagiolo et al. 2007; Moss 2008), we proceed with limiting the degree of complexity by assuming risk-neutral agents.

While expected utility is a predominant behavioral model to study the decisions under uncertainty, empirical evidence suggests that the households' decisions to buy a property could be made independent of risk. A number of surveys indicate that despite the fact that people realize they live in a flood zone and perceive flood risks as real, this awareness does not affect their choices when buying a house (Burningham et al. 2008; Willis et al. 20I I). Others suggest that people residing in flood-prone areas may just have biased risk perceptions. These individuals either do not realize that they bought a property in a flood zone (Chivers and Flores 2002) or they underestimate the objective flood probabilities (Ludy and Kondolf 2012). These subjective risk perceptions may change after a flood, increasing the housing price differentials, at least immediately following the flood (Atreya, Ferreira, and Kriesel 2013; Bin and Landry 2013). The question is how these perceptions of risks among individual households interact with the purchase of flood insurance. In order to address this question, we employ two extreme behavioral models: an expected utility model which assumes that people perceive risks objectively at all times (Eq. 4.4), and a risk neglect model with complete ignorance of flood risks, which assumes that households enjoy the utility of their properties as if there is no hazard involved (Eq. 4.I).

\footnotetext{
${ }^{17}$ In the first version of the model (Filatova 20I5) we used the traditional Cobb-Douglas utility function, therefore assuming that this function is concave and agents are risk-averse. While it is a useful assumption for theoretical modeling, bringing the agent-level decisions towards a more empirical basis required significant adjustments to the utility function (de Koning, Filatova, and Bin 2017).

${ }^{18}$ We ran a hedonic analysis on data from Bin and Landry (20I3) that includes 9,793 residential property sales between 1992 and 2002 to elicit preferences of individuals for different attributes.
} 
After a buyer has found the property that gives her maximum utility, she submits her bid price to a seller. Buyers' bid prices are anchored to sellers' ask prices varying within a range of -/+ 5 percent of an ask price of a property of interest. Price negotiations (Fig. 4.2) are modelled explicitly as an auction (Filatova, 2015). Buyers that are unsuccessful in securing a property will participate in a market in the next period. Buyers that are not able to find a property within their budget will leave the market.

\subsubsection{Sellers and adaptive price expectations}

Each time step a random number of properties goes for sale. At the beginning of a trading period, sellers announce their ask prices. Price expectation formation is a core process in any agent-based market. While ACE has made a major progress on modeling markets of homogeneous goods (Tesfatsion and Judd 2006), it remains challenging to model adaptive prices in property markets. The common ACE approaches to modeling price expectations in the bilateral trading environment do not fit housing markets well: spatial goods are highly heterogeneous and traders have little opportunities to learn efficient prices for such an infrequently purchased good as housing. The same house in a different location may have a disproportionately different price, as do two houses with different structural characterizes in the same neighborhood. Thus, modeling price expectations in housing markets relies on a mediator who regularly participates in transactions and learns what the appropriate price for any unique house may be. We introduce a real estate agent who observes successful transactions and re-estimates hedonic coefficients allowing to capture price changes due to shifting market conditions for different locations. In other words, the outcomes of the bilateral ACE market are registered in a database, which real estate agents analyze to understand the price trends on a market, as realtors do with Multiple Listing Service ${ }^{19}$ data in reality. In the model, every time step a real estate agent uses the simulated transactions of the past 6 months to estimate a new hedonic function. The new coefficients of this cross-sectional analysis are recorded into realtor's memory and are used to predict prices in the next time steps accounting for both the heterogeneity of spatial goods as well as the market dynamics.

\footnotetext{
${ }^{19}$ http://www.mls.com/
} 


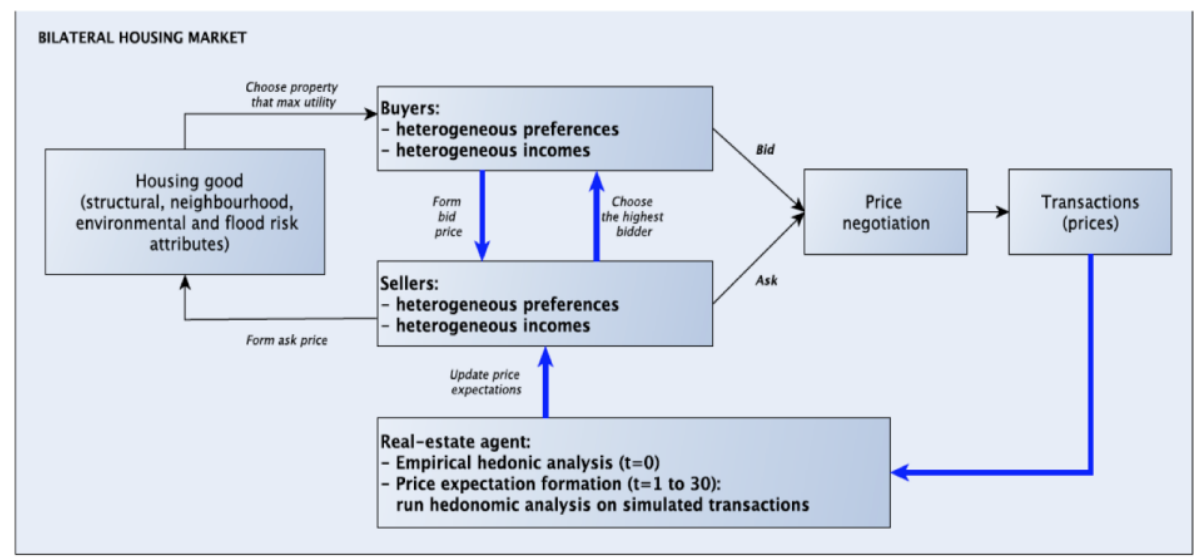

Figure 4.2. Agent-based housing market model

In summary, each seller forms an ask price for his unique property based on the implicit prices for various structural and neighborhood characteristics of his house, which come from the latest HA run by realtors. This ask price is also adjusted for the relative market power of traders when the real estate agent observes an excess demand for a particular segment of a market. A market segment is defined by properties that have similar characteristics and that are within a certain distance radius of each other. After all buyers make their choices, sellers select the highest bid price, if any, to engage in price negotiations (Fig. 4.2). Unsuccessful sellers decrease their ask prices in the future. Thus, the emerging shifts in housing prices are endogenously driven by the interactions of heterogeneous adaptive buyers and sellers. Due to the differences in households' incomes and preferences for neighborhood amenities across agents, some properties are more demanded than others. Thus, sellers of those properties will receive more bids, out of which they choose the highest, driving prices up. At the same time sellers with less or no bids are in the position to accept lower bids. Therefore, an allocation of heterogeneous spatial goods among households with heterogeneous incomes and preferences occurs endogenously via the market sorting. Note, that both demand and supply of residential properties are disaggregated in our HA-ACE model. It implies that if agents' preferences or their housing costs structure change - for example due to flood insurance premiums that make it more costly to live in a flood-prone area - it influences individual demand for locations. Cumulatively, this leads to endogenous changes in the aggregate market demand, and to the capitalization of these changes in property prices. 


\subsection{Results}

To quantitatively explore how insurance and households' risk attitudes affect floodprone housing markets we ran 4 experiments: (I) expected utility model without insurance, (2) expected utility model with insurance, (3) risk neglect model without insurance, (4) risk neglect model with insurance. We ran HA on simulated sales in each of the four experiments. Traditionally, HA is done only on one set of transactions over a certain time period. The discount for properties located in the flood zone is given by the coefficient of the 100-year and 500-year flood zone dummies in the HA. We perform multiple Monte Carlo runs of our four experiments, and hence we run the HA of the simulated transactions multiple times to come up with an average discount for properties located in the flood zone. We estimate the coefficients of the 100-year and 500-year flood zone dummies on the basis of ACE model sales in the last 5 years of the simulations ${ }^{20}$. The four experiments each performed 50 times deliver 200 hedonic regression results, providing us with a set of 200 flood dummy regression coefficients that differ among the four experiments. We compare the average flood dummy coefficients by running an ANOVA on these coefficients (= dependent variable), with the experimental settings and their interaction (risk attitude, insurance policy, risk attitude with insurance policy) as independent variables.

To assess how risk attitudes and insurance premiums capitalize into property prices separately, we compare the four experiments within the ANOVA. None of the coefficients are measured on the basis of their absolute value, for the following reason: we find a baseline price discount for properties located in the flood zones that cannot be attributed to either capitalized insurance rates or capitalized risk attitudes. We assume that a discount for properties in the flood zone reflects people's perception of risk, and the capitalization of flood insurance. Yet, when risk perceptions and flood insurance equal 0 , we still find a discount for properties in the flood zone in our simulation experiments (Table 4.2, second column) where there should be none. This discount reflects the mean coefficient of the flood zone dummy in the experiments with risk neglecting buyers and without the full insurance uptake. In fact, the second column in Table 4.2 is derived from the intercept in our ANOVA, where both factors (risk attitude and insurance) are equal to 0 (Appendix 4.b outlines

\footnotetext{
${ }^{20}$ We limit our analysis to the last 5 years of the simulations to focus on the period when the agent-based housing market has converged to an equilibrium. ACE models are inheritably dynamic models, which may or may not converge to an equilibrium or a set of equilibria. Our agent-based housing market model does converge to an equilibrium, meaning that the market price stabilizes.
} 
how the percentages in Table 4.2 are calculated). In our ANOVA, we compare the differences in the coefficients of the flood zone dummy among the experiments, while the coefficients in all experiments deviate from zero statistically significantly - and hence the intercept is significantly different from zero. The value of the intercept is -0.261 and -0.226 in the 100-year and 500-year flood zones respectively. We consider this as the baseline price discount for properties located in the flood zone compared to properties outside the flood zone. This discount distorts our estimates of the flood dummy coefficient in the simulations, and is likely governed by spatial correlation between location in the flood zone and other housing attributes. More on this is explained in detail in de Koning, Filatova, and Bin (2017).

We discuss the results of the integrated HA-ABM model in line with two issues. Firstly, we identify the pure capitalization of flood insurance premiums in housing prices under the hypothetical scenario of the full insurance take up. Secondly, we compare the effect of flood insurance with the effect that the risk neglect behaviour may have on housing values. Our agent-based model offers a virtual lab, in which we experiment with insurance premiums and households' risk attitudes. The combination with the hedonic pricing method allows us to identify separately the marginal contribution of risk attitudes and flood insurance to the prices of properties in the flood zone. Moreover, we show other relevant variables in a housing market that our agent-based model enables us to analyze on top of the hedonic price analysis. The results are discussed step by step below.

\subsection{Discount on property prices in the flood zone due to insurance}

Firstly, we look at the flood dummy coefficients based on HA of simulated transaction prices with and without insurance. We compare the means of the coefficients to estimate how much the property values in the flood zones would decrease because of the flood insurance premium with the full insurance uptake. In the simulations, buyers take the insurance costs into account in their housing budget, and thus it reduces the amount that buyers spend on housing. This is not the case in the simulations where the insurance is absent. Thus, the comparison between the coefficients of these scenarios highlights the marginal implicit price of insurance costs, which add to buyers' budget constraints and capitalize into the housing price in the flood zone. 
Table 4.2. Summary stats for the flood zone coefficient

\begin{tabular}{lllll}
\hline $\begin{array}{l}\text { Flood } \\
\text { zone }\end{array}$ & $\begin{array}{l}\text { Average price } \\
\text { compared to safe zone }\end{array}$ & $\begin{array}{l}\text { Discount for } \\
\text { flood insurance }\end{array}$ & $\begin{array}{l}\text { Discount for } \\
\text { flood risk }\end{array}$ & $\begin{array}{l}\text { Flood risk * } \\
\text { insurance }\end{array}$ \\
\hline I00-year & $-23.0 \%$ & $-8.1 \%$ & $-6.2 \%$ & $+5.2 \%$ \\
& $(+/-0.7 \%)$ & $(+/-1.0 \%)$ & $(+/-1.0 \%)$ & 0 \\
500 -year & $-20.2 \%$ & $-4.4 \%$ & 0 & 0 \\
& $(+/-0.6 \%)$ & $(+/-0.8 \%)$ & & \\
\hline
\end{tabular}

The third column in Table 4.2 shows how flood insurance capitalizes into property values in the 100-year and 500-year flood zones. In the case of full insurance uptake, we see that the average price of a property is reduced by $-8.1 \%(+/-1.0 \%)$ and $-4.4 \%$ $(+/-0.8 \%)$ on average in the 100 -year and 500 -year floodplains respectively, irrespectively of how individuals perceive risks. In other words, the coefficient of the flood zone dummy is on average reduced by $0.085(+/-0.010)$ and $0.045(+/-0.008)$ in the 100-year and 500-year flood zone respectively as a result of insurance premiums that capitalize into property values. Equations A4-All in Appendix 4.b explain more in depth how the difference in the coefficients of the flood zone dummy between the simulations are used to calculate a percentage discount for a property in the flood zone driven by the insurance costs.

In addition, we also explore parameters in the model that explain the discount for properties with the full insurance take up. As described before, insurance premiums add to the costs of flood-prone properties affecting the budget constraint of buyers. It makes properties in the flood zone less affordable. That is, a property in the flood zone, which gives the highest utility for a certain buyer, will likely be close to the maximum budget for that buyer. Thus, this property may become unaffordable when insurance costs are included. At the same time, higher income buyers who could afford that particular property will most likely avoid it because it does not maximize their utility: for the same housing budget they can afford a better house avoiding additional insurance costs. This leads to a decrease in demand of properties in the flood zone followed by the need for sellers to lower ask prices for properties that require flood insurance. These feedback effects make properties in the flood zone more affordable again over time for the former group of buyers, since they would have to spend less of their budget on the housing. Indeed, looking at the relative amount that buyers spend on a house, we see that buyers of properties in the 100 year flood zone spend on average $88.4 \%$ of their maximum housing budget ${ }^{21}$ on a

\footnotetext{
${ }^{21}$ The maximum housing budget is the maximum amount that buyers can afford to spend on housing. In most cases they end up spending less than this amount. In the simulations they spend on average about
} 
house when they do not need a flood insurance, while they spend on average $83.7 \%$ of their housing budget when required to purchase a flood insurance. Thus, buyers in the 100-year flood zone spend on average $4.6 \%$ less on housing when they need to incur additional cost on flood insurance. In the 500-year flood zone buyers save $3.1 \%$ of their budget on housing under the full insurance take up scenario.

\subsubsection{Separating the effect of risk attitudes from the effect of flood insurance}

Next, we compare the expected utility model with the risk neglect model. It allows us to calculate how risk attitudes capitalize into property prices and to quantitatively examine an interplay between imperfect information about risks among households and the flood insurance. The factor "risk attitude" in the ANOVA (Table 4.2, column four) shows the discount for properties in the flood zone that can be attributed to the capitalization of risk attitudes among the buyers. The expected utility model differs from the risk neglect model by the way buyers perceive flood risk and take it into account when searching for a property that maximizes their utility under budget constraints. Buyers in the risk neglect model do not consider the flood zone status of a property in their utility calculations, while buyers in the expected utility model reduce their utility by the objective probability of flooding, multiplied by expected damages. These perceived losses in utility decrease the demand of informed buyers for properties in the flood zone under the expected utility model, leading to a reduction of property prices therein. Hence, the mean coefficient of the flood zone dummy is lower in the expected utility model than in the risk neglect model. Table 4.2 (fourth column) shows that the expected utility model lowers the value of 100 year flood zone properties by $-6.2 \%(+/-1.0 \%)$ compared to a housing market where buyers ignore flood risks. In other words, when buyers perceive risks objectively, the price of properties in the flood zone is reduced by $-6.2 \%$ on top of the discount for flood insurance premiums. These results show that prices for properties in the flood zone depend on how buyers value the risk of living in the flood zone. When buyers neglect flood risks in a market, the aggregate flood risk discount is less than it would be in market with traders who are perfectly informed and have neutral risk attitudes. Generally, a higher risk perception leads to a larger discount for properties located in the flood zone. We can use the simulations in our ABM to quantify the marginal implicit price of flood risk in the market (de Koning, Filatova, and Bin , 2017). Risk attitude had no significant effect on property values in the 500-year flood zone,

$88-89 \%$ of this amount, but it varies depending on whether insurance premiums are also considered by buyers. 
likely because the probability of flooding is too small to have a relevant impact on the price.

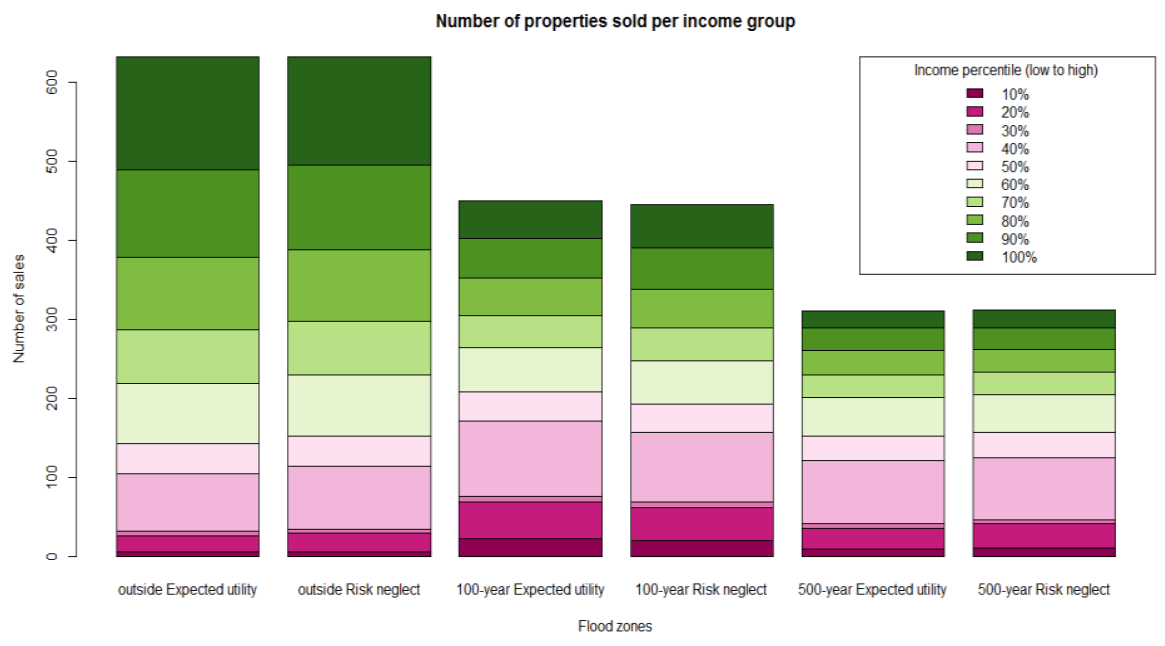

Figure 4.3. Properties sold in each flood zone, distributed in household income percentiles

Again, we look at various other measures in the model to see how the discount for properties in the flood zone due to risk manifests itself in the housing market. Fig. 4.3 shows how property sales in the 100-year and 500-year flood zone and outside the flood zone are distributed among income groups in the expected utility model and risk neglect model. It appears that the 100 -year flood zone is relatively highly represented by the 20 percent lowest income homeowners, while properties outside the flood zone are relatively highly represented by the 30 percent highest income households. Hence, the discount for properties in the 100 -year flood zone results in a larger number of low income households that occupy these properties. Comparing the expected utility model with the risk neglect model, we also find that there are slightly more high income households (highest 10\% in particular) in the 100-year flood zone when people ignore the risks. In the expected utility model we see slightly more low income (lowest 40\%) households in the 100-year flood zone. This pattern is initially driven by the reduction of property prices resulting from lowered demand given buyer's risk attitudes. Further, due to the market sorting lower income households are allocated to the areas where property prices are lower, leading to the path dependence.

In addition to the individual effect of the two main factors (risk attitude and insurance) in the ANOVA on the coefficients for the 100-year and 500-year flood zone dummies, we also find a significant interaction effect in the 100 -year flood zone. 
Full insurance take-up in combination with the expected utility model increases the mean price of a property in the 100 -year flood zone by $+5.2 \%(+/-1.5 \%)$ (Table 4.2 , fifth column), leading to the overall discount of $-9.3 \%$ for flood zone properties. While insurance premiums and flood risk both capitalize into property prices leading to a discount for properties in the 100 -year flood zone (reducing the value of a property by $-8.1 \%$ and $-6.2 \%$ respectively), we also see that expected damages are lower in the expected utility model with insurance than in the expected utility model without insurance. Hence, the discount for properties in the flood zone in the simulations with expected utility traders and the full insurance uptake is less than the sum of the effects. Flood insurance comes with additional costs for an individual homeowner in the form of annual premiums, as well as with additional benefits in the form of damage coverages in the case of a flood. The fact that insurance coverages compensate (part of) the damage alters buyers' utility, which capitalizes positively into prices for properties in the 100-year flood zone. Thus, despite the fact that maximizing the expected utility among traders result in an additional discount for properties in a flood zone, we find that this discount reduces with the full insurance take-up, as anticipated in theory (Frame, 1998).
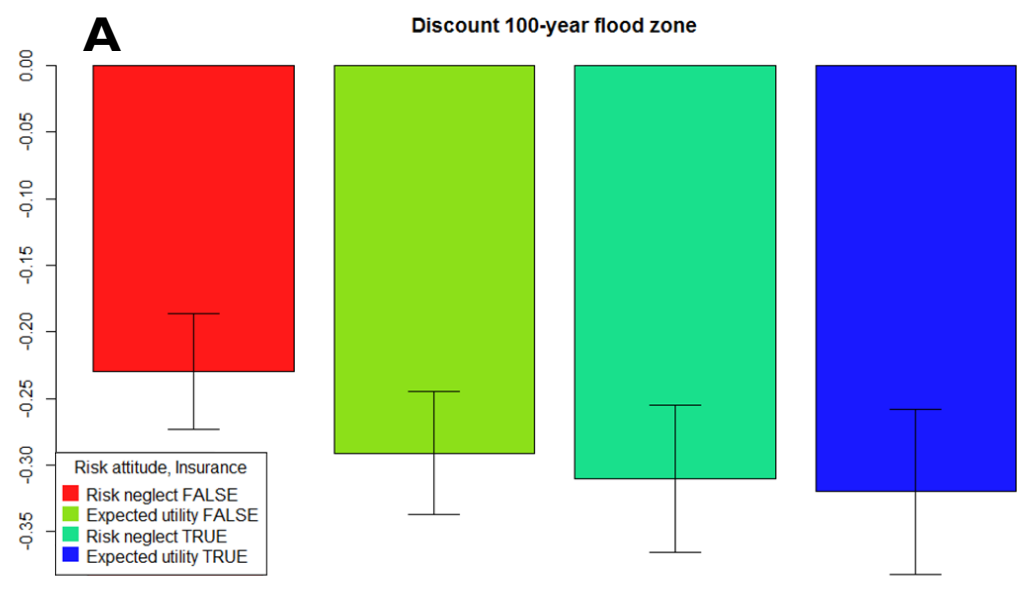

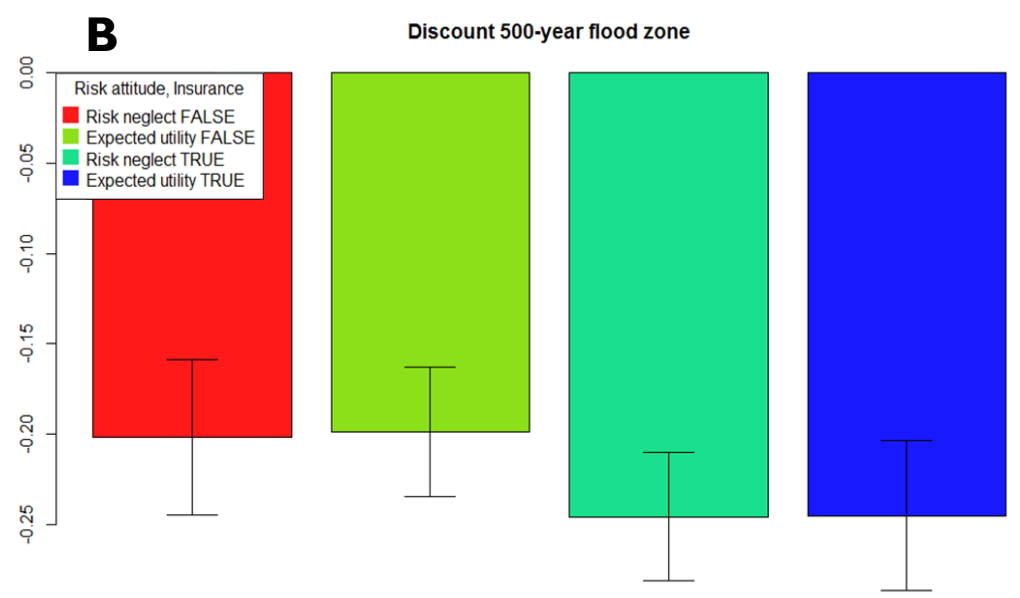

Model

Figure 4.4. Discount for Properties in the 100 -year $(A)$ and 500-year (B) flood zone

Fig. 4.4 shows the mean coefficients (+/- one standard deviation) of the 100 -year flood zone dummy $(A)$ and 500-year flood zone dummy $(B)$ of the four simulations. It illustrates that flood insurance consistently causes a lower price for properties in both the 500-year and 100-year flood zone, independent of buyers' risk attitudes. Empirical work finds that price discount changes over time as individual risk perception of market participants is magnified after a flood event (Atreya, Ferreira, and Kriesel 20I3; Bin and Landry 20I3). Our agent-based model integrated with HA allows us to consider two extremes at the individual household level - fully informed traders with risk-neutral attitudes and individuals who entirely ignore these risks and to study how market outcomes change in each case. Our analysis indicates, that irrespectively of whether individuals perceive risks objectively or ignore them completely, a hypothetical scenario of the full flood insurance take up leads to the price decrease in the flood zone. Lower value for the capital at stake emerging in a flood-prone housing market has two positive implications. Firstly, an individual choosing to buy a house in a flood zone is compensated for the additional insurance costs by paying a lower price for the hazard-prone house. Secondly, lower property values in flood zones translate into less direct damages in the case of a flood, leading to less pressure on the NFIP and eventually taxpayers, as well as to less incentives to redevelop in the risky zone (Table 4.3). Hence, these results may provide important policy implications. 
Table 4.3. Total housing capital at risk in the 100 -year flood zone

\begin{tabular}{llll}
\hline Experiment settings & \multicolumn{2}{l}{ Insurance } \\
\cline { 3 - 4 } & & False & True \\
\hline Risk attitude & Risk neglect & $\$ 158.4 \mathrm{M}$ & $\$ 150.5 \mathrm{M}$ \\
& Expected utility & $\$ 152.3 \mathrm{M}$ & $\$ 148.6 \mathrm{M}$ \\
\hline
\end{tabular}

\subsection{Discussions and conclusions}

This paper contributes to the existing literature on flood insurance by exploring the effects of a hypothetical policy and by illustrating a methodological value of combining HA with simulations. Firstly, we assess the impact of flood insurance on property prices under various risk attitudes in a hypothetical scenario of $100 \%$ take-up. Secondly, we intend to show the usefulness of $A B M$ as a tool to address such challenges that require disentangling different policy effects in a market. We conclude with critically reflecting on the challenges we still face with this approach and suggest opportunities for future work.

\subsection{Policy impacts: effect of insurance and flood risk on property values}

The results from our agent-based model grounded in HA show that insurance reduces the property values on average by $-8.1 \%$ and $-4.4 \%$ in the 100 -year and 500 year flood zone respectively. We compare the discount for properties in a flood zone in a scenario with $100 \%$ insurance uptake and a scenario without insurance. The effect is consistent, independent of households' risk attitudes implying that the flood zone discount is present even if risk is ignored among buyers. This shows that flood insurance serves as a mechanism to convey risk information to buyers independently of risk attitudes or a potential flood risk information asymmetry, ensuring that the flood risk is capitalized into property values. Our simulation is based on the assumption of full compliance, but in reality some homeowners still may choose not to be covered by the insurance. The net effect then should depend on the enforcement effort and the compliance to the proposed change.

We find that risk attitudes also affect property values in addition to the insurance effect, but its effect on property values is less than the capitalized effect of insurance. When people are risk-neutral, the value of properties in the 100 -year flood zone is reduced by another $-6.2 \%$ on top of the insurance effect. We find no effect of risk attitude in the 500-year flood zone. Given that expected damages in the 500-year flood zone are rather low, it is likely that the effect is too small to yield a significant effect on property prices in the 500-year flood zone. 
Furthermore, we find that the insurance reduces the effect of risk perceptions in the expected utility model. Expected damages are reduced because they are (at least partly) covered by the insurance, making people feeling less at risk should a flood occur. The damages that are covered by flood insurance capitalize as an increase in property values by $+5.2 \%$ in the 100 -year flood zone in the expected utility model with insurance relative to the case with the complete ignorance of risks, both still delivering a flood zone price discount of $-8.1 \%$ and $-9.3 \%$ correspondingly. This minimizes the difference between the expected utility model and risk neglect model in case of $100 \%$ insurance uptake, which has important policy implications. Risk perceptions may be biased depending on information and experience with flooding events (Atreya, Ferreira, and Kriesel 20I3; Bin and Landry 20I3). Experience drives changes in people's risk attitudes, and hence the discount for properties in the flood zone is not stable over time. This implies that the value of capital at stake is very dependent on the volatility of homeowners' risk attitudes, unless there is a market mechanism in place that stabilizes the value of properties in the flood zone over time (Bagstad et al. 2007; Filatova 2014). The results in our simulation study suggest that the full insurance uptake may provide such a mechanism for two reasons: I) the value of insurance premiums capitalizes into property prices, thereby reducing the total value of capital at stake, and 2) the insurance may reduce people's risk perceptions because (part of) the expected losses are covered by future insurance claims.

\subsubsection{Methodological lessons: benefits of agent-based computational modelling and open challenges}

The issues addressed in this paper represent several scientific challenges. Namely, (I) quantifying the impact of insurance and risk in hazard-prone dynamic markets (MacDonald et al. 1990; Harrison et al. 200I; Bin, Kruse and Landry 2008), (2) isolating the marginal impacts of individual housing attributes in a housing market where many of these attributes are spatially correlated, (3) dealing with risk attitudes that change over time (Bin and Polasky 2004; Atreya, Ferreira, and Kriesel 2013; Bin and Landry 2013) and (4) addressing the issue that property values in hazard zones are pushed up by the demand for environmental amenities and people that ignore the risks (Bin, Kruse and Landry 2008). The results of our model, that integrates HA and agent-based simulations illustrate the usefulness of the method to address these scientific challenges.

While we try to rely on empirical data and secondary literature sources as much as possible here, the numbers presented in this paper should not be taken literally for a policy analysis or for drawing any direct policy implications. This should rather be considered as an illustrative case, which shows the possibilities of the method that 
combines traditional empirical HA and computational economics simulation models to directly trace market impacts of a policy intervention under adaptive market dynamics. By combining the strengths of two methodologies we are able to run experiments and quantify the impacts of the complete take-up of flood insurance which alters households' budgets constraints. Although hedonic studies have demonstrated a link between insurance premiums and price discounts, the innovation of our approach is in its ability to trace the marginal contribution of flood insurance and risk in isolation, as well as their interaction. We provide an experimental landscape in which we can vary certain parameters while keeping others constant and vice versa. We can systematically run multiple simulations under the same settings to estimate a mean effect of various factors on property values in the flood zone. In contrast to hedonic studies in real markets that are limited to a single time series of sales, our agent-based housing market model can produce multiple time series under the same set of assumptions. This way we can eliminate stochastic effects caused by availability of properties on the market and shifting preferences of buyers over time. We can also work around spatial correlation in housing attributes by comparing outcomes between sets of experiments. Moreover, in scenario studies we can use our model to explore policy options that could be adopted to inhibit price changes caused by changing risk attitudes. In the hypothetical scenario of the $100 \%$ insurance uptake we explore how this affects the value of capital at stake.

Finally, in our agent-based simulations we can assess other relevant market parameters, such as households' budgets spent on housing and income distributions among different housing sectors, which allows us to go beyond HA and study the impact of certain policies on the market in more detail. Agent-based computational economic models trace the emergence of aggregated market level features, such as market demand or prices and number of sales, as well as provide an opportunity to record relevant disaggregated data such as incomes or preferences of traders. It allows a unique analysis of macro-level phenomena and patterns using micro-level data (Sun et al. 20 I4; Filatova et al. 20II).

\subsubsection{Challenges and future work}

Several caveats are in order for our analysis. First, our initial HA is based on a township in Carteret County while the income variable comes from general US public and the preference variable are derived from Pitt County in North Carolina. Albeit spatially close, the simulated demand in our model is not of the same population from the hedonic analysis of Bin, Kruse, Landry (2008). We do not consider this problematic for the purpose of this research because the results should 
in any case be interpreted as a representative example, rather than a case-specific study. Yet, it may partially explain why we find such a large discount for properties in the flood zone in the experiment with risk neglecting buyers without insurance (Table 4.2, second column). Namely, the preferences of buyers in our simulations are not exactly in line with the preferences of actual buyers in our housing transactions dataset. At initialization we obtain our data from a market that is structured according to preferences and budgets of one set of buyers while during our simulation the preferences and budgets of another set of buyers capitalize into property values. Although the hedonic coefficients update at each time step to match with buyers' preferences, there is a remaining bias in the coefficient estimates that amplifies the discount for properties in the flood zones, which is caused by multicollinearity in housing characteristics (de Koning, Filatova, and Bin 2017). Hence, the hedonic analysis in our agent-based simulations can only be useful when it is applied in a comparison between multiple experimental scenarios.

Second, our model requires disaggregated empirical data on buyer preferences for different housing and location attributes. We tried to disaggregate buyers' preferences by inferring them from transactions in an actual market through the ANOVA. Since the strength of agent-based modeling lies in simulating the aggregated behavior of a system that emerges from individual choices, preferences and interaction of individuals (Farmer and Foley 2009; Tesfatsion 2006; Tesfatsion 2017). Hence, one needs data on individual preferences to simulate how macro patterns emerge from the bottom up, rather than attempting to infer preferences of individuals from the emerging system. We strived to move away from theoretical modeling, bringing the agent-level decisions towards a more empirical basis, in which we chose to compromise in the complexity of the theoretical models. In an attempt to infer buyers' preferences with empirical data, we simplified the utility function by assuming a linear risk-neutral behaviour, while the typical shape is concave, representing the risk-averse behaviour. In order to properly capture individuals' riskaverse (or risk-seeking) behaviour, the degree of concavity or convexity of these utility functions too need to be parametrised with empirical data. Given the complexity and the many interactions between individuals that give rise to the behavior of a system, any errors in the modelled behavior may propagate through the system. Intensive Monte Carlo simulations are a must to assure the reliability of agent-based market estimates. Yet, any errors in the underlying micro data may naturally lead to amplified errors on the macro scale.

Third, our simulation is based on the assumption of the full insurance compliance, but in reality some homeowners still would choose not to be covered by the 
insurance. The net effect of a program that stimulates insurance uptake should depend on the enforcement effort and the compliance to the proposed change.

This work could be extended in several directions. Firstly, a realistic policy analysis requires more precise data on insurance premiums and coverage as well as disaggregated data of preferences for amenities and housing goods. It may require a survey that explicitly studies risk preferences and perceptions of sellers and buyers and elicits any behavioral rules underlying their location choices. Secondly, as discussed by Viscusi (1985) and Smith and Desvousges (1988) people constantly learn about risks they face. We simulated two models of individual risk attitudes in a static way. In reality, risk perceptions change over time as people forget or get reminded about a specific hazard such as flood in our case, which can seriously alter their locations choices and willingness-to-pay for safety. Empirical research on housing markets in flood-prone areas captures that there are indeed dynamics in flood risk perceptions, which get exacerbated just after a disaster and get forgotten over time, as reflected in price discounts changing dramatically over time. Our ACE model integrated with traditional hedonic analysis could be used to study how the flood risk capitalization in housing prices changes over time as people's risk perceptions get updated or vanish, and assess how the insurance could be used to interfere with these dynamics. Thirdly, as such changes in individual behavior could be captured and parameterized with data, one may explore non-marginal effects, which are anticipated in economic systems in climate change world (Stern 2016). As opposed to majority of economic tools that are designed to study gradual changes along the same trend, computational economics models are not bounded to such marginal dynamics. This could potentially open new methodological opportunities and shift research frontiers to quantitatively examine non-marginal changes as well. 




\section{5.| Introduction}

Flooding is the most frequent and consequently also one of the costliest natural disasters. In the U.S. alone it causes $\$ 7.96$ Billion in damages per year on average (NWS 2018). With changing climate its impacts are expected to grow due to more frequent extreme rainfall events and sea level rise Jongman et al. 2012, UNISDR 20II, Wensemius et al. 2016). Therefore, climate adaptation policies focus on effective strategies to reduce adverse impacts of increasing flood risk (Dronkers et al. 1990). To be effective in increasing resilience against flood risks, climate adaptation policies should span across scales (Adger et al. 2005) making it important to understand the endogenous bottom-up drivers of societal vulnerability to flood risk. How is flood risk perceived by individual households? What motivates homeowners' protective responses to flood risk? Are people prepared to move away from hazardprone areas when the risk becomes too high? Does it differ among people who are already in the flood-zone compared to those who are still choosing a place to live? Answering these questions requires an understanding of people's perception of the hazard, and the cognitive processes that drive their preparedness to adopt protective measures and adapt to increasing risk.

Behavioural science offer a rich set of theories that elaborate on these cognitive processes behind individual decision making. Potentially, each of them may lead to different hypotheses about how people choose to protect themselves against flood risk. The probability of flooding and anticipated damages, the two main variables of expected utility theory, are the most common factors in explaining what motivates risk management behaviour (Bubec et al. 2012). Expected utility theory postulates that people weigh the costs of living in a hazard zone (and of adopting risk mitigation measures) by the probability of a flood occurring, and the damages associated with a flood of a certain reoccurrence interval. In practice, the probability of a flood and its expected impact could be perceived subjectively, vary between different people, and can change over time in response to experience with floods and exchange of information. A more extensive theory which links risk appraisal with coping appraisal is protection motivation theory (Grothmann and Reusswig 2006, Kahnemann and Tversky 1979), adding behavioural variables such as perceived control efficacy, perceived self-efficacy and perceived control costs. This theory suggests that in order to consider risk management strategies people should perceive a minimum level of risk. If they do, they proceed with weighing the costs and effectiveness of the risk management strategies, as well as their perceived control over the risk. Protection motivation theory also encompasses non-protective responses, such as fatalism or denial, that negatively motivates protective responses. Another psychological theory 
on risk mitigation behaviour is the psychometric paradigm or the affect heuristic, which focuses on decision making driven by feelings of dread and fear (Slovic et al. 2005). The latter is also included in protection motivation theory. Some of these theories focus on perceptions alone, like the affect heuristic (Slovic et al. 2004) and the psychometric paradigm, while others (such as protection motivation theory) include behavioural responses as well. We explore a multitude of variables that are relevant in households' motivation to adopt various risk management measures, including the choice to evade the hazard zones. We empirically assess the effects of the variables of multiple theories to examine what drives households' decisions at multiple stages, and how this affects their vulnerability to flooding. We use the variables of protection motivation theory as the basis, because it is the most elaborate framework and the most widely used in explaining homeowners' risk mitigation behaviour (Bubeck et al. 2012, Grothmann and Reusswig 2006). Moreover, it includes most of the variables that are present in other theories as well.

There are numerous empirical studies grounded in various theoretical frameworks that attempt to explain risk mitigation behaviour in response to flooding (as reviewed by Kellens et al. 2013, Bubeck et al. 2012, and Koert et al. 2017). Affect heuristics and the psychometric paradigm are frequently studied (Ge et al. 20I I, Ho et al. 2008, Keller et al 2006, Terpsta 20II) as well as concepts from protection motivation theory (Grothmann and Reusswig 2006, Poussin et al. 2014, Zaalberg et al. 2009). Most of these behavioural theories have been used to explore individual choices when facing flood risks (Bubeck et al. 2012, Kellens et al. 2013, van Valkengoed and Steg 2019) assuming households take only incremental actions such as taking flood insurance. In light of climate change, however, transformative adaptation actions such as relocation from a flood zone - will become prominent (Kates et al. 2012). In the long term, planned retreat - as opposed to flood protection (e.g. levees, dikes, seawalls) or damage mitigation (e.g. flood proofing, drainage systems) - is a viable policy option to overcome the increasing costs of flood protection and increasing flood damages (Song et al. 2018, Filatova 2014). The implications for society of such a high-impact policy as planned retreat has been studied for example by Freudenberg et al. (20I6), including on what drives public support (see: Alexander et al. 2012 and Rey-Valette et al. 2019). Yet, it requires major planning and is not necessarily supported by the affected households. Given that publicly planned retreat is a major political intervention, it would take many years to operationalise. Meanwhile retreat as a private decision of households may become increasingly prominent, in particular following a major flood event such as after hurricane Katrina (Zaninetti and Colten 2012), which puts high-income households in a favourable position over low-income households, who may find themselves trapped due to the lack of resources to move 
(Black et al. 20I3). Yet, while evading flood zones is an effective way of reducing the risks, the choice to live there is fuelled by a desire to live close to water or have an ocean view. Having rich environmental amenities may result in up to a $36 \%$ premium for properties that are actually located in a flood zone (Beltrán et al. 2018). The expansion of urban development into hazard-prone areas is the largest driver of risks in a climate changing world, not the growing probabilities of hazards (IPCC 20I2, Wong et al. 2014). Hence, it is important to understand what cognitive mechanisms drive the balance between the costs of living in a hazard zone and enjoying the benefits of coastal locations including environmental amenities. Will an increase in hazard events shift this balance and prompt a household to move out of a floodzone?

What drives individual households to reconsider their location choice and to relocate to higher ground has received little attention. To the best of our knowledge the only exception is the work of Treuer et al. (2018). This is however based on a lab experiment, which does not provide empirical evidence for people's preparedness to move when it concerns their own home. To address this gap, we run a survey among 1040 households in coastal hazard-prone areas in the U.S., of which some have recently experienced a major flooding. We study what behavioural mechanisms drive the relationship between flood mitigation and outmigration separately among property buyers and sellers, who shape the demand for locations - and consequently future urbanization patterns - in flood-prone cities. Therefore, the survey covers the choice of location where to live, how people protect themselves against river and coastal flooding, and how this impacts their vulnerability to flooding and possible future impacts due to climate change. It aims to assess (I) how households respond to flood risk in terms of choosing where to live and how to protect themselves against flooding, (2) which factors change households' perceptions towards flood risk and how their perceptions affect their adaptation choices, and (3) how households' responses to flood risk potentially impacts their vulnerability to flooding in a changing climate. Studies on homeowners' flood risk management, including many surveys, focus primarily on protective measures such as flood insurance and flood proofing (Bubeck et al. 2012, Bubeck et al. 2013, Grothmann and Reusswig 2006, Terpstra 20I I, Zaalberg et al. 2009). They employ cognitive decision making models such as protection motivation theory as well as theories of affective decision making to explain why some people protect themselves and others do not. This article adds to the literature by including location choice as a protective response to flood risk. Those who live in areas that are prone to flooding can choose to adopt protective measures. Yet, a natural line of defence against flooding is choosing not to live in these areas in the first place. Risk is a product of 
probability and exposure, meaning that households can mitigate risks by altering both. Households can minimise the probability by adopting measures that reduce damage to structure and content in case of flooding, or they can minimise their exposure by moving out of the flood zones or avoiding buying a home in these areas. The latter will become increasingly important in the context of climate change when sea levels rise and flood hazards increase. As flooding events become more frequent and severe and exposure increases due to urbanization (Jongman et al. 2012, UNISDR 20II, Wong et al. 20I4), local flood climate adaptation measures become more costly and less effective. It brings attention to adaptation by avoiding areas with increasing hazard either through planned retreat or bottom-up relocation. Our survey assesses how information, learning and experience with flooding alter households' responses to flood risk and the individual factors (affective and cognitive) that drive the latter. Our study includes some unique data because our sample includes the respondents who were affected by Hurricane Harvey of 2017. By focusing on how buyers and sellers respond to flooding events, we address the choices of potential newcomers to flood risk areas (buyers) as well as of people who are already there (sellers) but could take a decision to move away from the hazard.

\subsection{Data and methods}

\subsection{Study area and data}

This study focuses on two types of flooding that are both caused by hurricanes river and coastal flooding. Our study area consists of 8 states in the U.S. along the Gulf and Atlantic coast that are known to have periodic flooding from hurricanes: Texas, Louisiana, Mississippi, Alabama, Florida, Georgia, South Carolina and North Carolina. Our sample was focused on areas close to the coast and waterways, focusing on those areas where flood risk is most relevant. We conducted the survey as an online questionnaire ${ }^{22}$ in January and February 2018 among buyers and sellers separately who were either active on the market at the time of the survey or had recently bought or sold a property in the study area. The buyer and seller survey were sent out to different people, and thus no respondent could fill in the survey in both roles as seller and buyer. The identification of active buyers and sellers was done through a series of qualifying questions: 'are you currently selling your house?', 'are you currently in the market to purchase an owner-occupied house?' and 'have you identified

\footnotetext{
${ }^{22}$ The sampling was assisted by a U.S. survey company 'Survey Design \& Analysis' through their georeferenced databases.
} 
a property on which you are considering making or have made an offer?' (Appendix 5.b and Appendix 5.c, part I). We aimed to sample as many active buyers and sellers as possible. Furthermore, we included households that had bought or sold their residential property in the past 12 months to increase our sample size. This group responded affirmatively to the questions 'did you purchase the home you live in within the past 12 months?' or 'have you sold your house within the last twelve months?'. Of the responding buyers only $12.5 \%$ were active in the market, while $42.0 \%$ of the responding sellers were active on the market at the time of the survey. It proved to be challenging to sample buyers who were active on the market, particularly because the survey included specific questions about a property they were likely going to buy. This required buyers that already had a specific property in mind on which they were considering making or had made an offer. Other buyers that had no interest in any specific property disqualified for the survey, which reduced the number of active buyers in our survey.

Table 5.I shows the number of buyer and seller responses, where they come from and their household income. The majority of respondents are from Florida and Texas which have been hit by Hurricane Matthew in October 2016 and Hurricane Harvey in August 2017 respectively. Hurricane Harvey caused severe rainfall and is ranked as the second costliest hurricane on the U.S. mainland after Hurricane Katrina, causing $\$ 126$ billion worth of damages (National Hurricane Center 2018). A total of 72 respondents in our survey $(6.7 \%)$ had experienced damage from a flood.

Table 5.I. Demographics of survey respondents

\begin{tabular}{llll}
\hline & Buyers & Sellers & Combined \\
\hline Number of responses & 519 & 521 & 1040 \\
Active on the market & $12.5 \%$ & $42.0 \%$ & $27.3 \%$ \\
State: & & & \\
Alabama & $6.6 \%$ & $6.5 \%$ & $6.5 \%$ \\
Florida & $30.6 \%$ & $27.8 \%$ & $29.2 \%$ \\
Georgia & $9.6 \%$ & $11.3 \%$ & $10.5 \%$ \\
Louisiana & $5.4 \%$ & $4.0 \%$ & $4.7 \%$ \\
Mississippi & $1.7 \%$ & $3.8 \%$ & $2.8 \%$ \\
North Carolina & $12.9 \%$ & $15.0 \%$ & $13.9 \%$ \\
South Carolina & $8.9 \%$ & $7.5 \%$ & $8.2 \%$ \\
Texas & $24.3 \%$ & $24.0 \%$ & $24.1 \%$ \\
Flood zone: & & & \\
Outside & $60.1 \%$ & $67.4 \%$ & $63.8 \%$ \\
I00-year recurring flood & $6.0 \%$ & $9.0 \%$ & $7.5 \%$ \\
$500-y e a r$ recurring flood & $6.0 \%$ & $5.8 \%$ & $5.9 \%$
\end{tabular}




\begin{tabular}{llll}
$\begin{array}{l}\text { Unknown } \\
\text { Experience with }\end{array}$ & $27.9 \%$ & $17.9 \%$ & $22.9 \%$ \\
flooding & $24.7 \%$ & $25.7 \%$ & $25.2 \%$ \\
$\begin{array}{l}\text { Household income: } \\
\text { Less than } \$ 30,000\end{array}$ & $11.0 \%$ & $9.6 \%$ & $10.3 \%$ \\
$\$ 30,000-\$ 49,999$ & $16.4 \%$ & $12.7 \%$ & $14.5 \%$ \\
$\$ 50,000-\$ 74,999$ & $22.5 \%$ & $24.7 \%$ & $23.7 \%$ \\
$\$ 75,000-\$ 99,999$ & $19.5 \%$ & $23.6 \%$ & $21.5 \%$ \\
$\$ 100,000-\$ 149,999$ & $19.8 \%$ & $17.3 \%$ & $18.6 \%$ \\
$\$ 150,000-\$ 199,999$ & $4.2 \%$ & $5.4 \%$ & $4.8 \%$ \\
$\$ 200,000$ or more & $3.7 \%$ & $2.5 \%$ & $3.1 \%$ \\
Prefer not to answer & $2.9 \%$ & $4.2 \%$ & $3.6 \%$ \\
\hline
\end{tabular}

\subsubsection{Variables of interest and ways to measure them}

Our primary interest is in revealing behavioural factors that drive a major choice of homeowners among various flood risk management strategies: to protect property from flood damage when choosing flood-prone locations or to avoid properties in hazard locations entirely. Various behavioural factors, including people's experience with flooding, were formulated into specific variables (Table 5.2). Subsequently we selected the questions from the survey that measure these variables (shown in the second column of Table 5.2). The questions in the survey have been carefully formulated in line with existing surveys (e.g. Van Duinen et al. 2015, Lindell and Hwang 2008, Bubeck et al. 2013). The buyer and seller survey consisted of 37 and 44 questions respectively. We ended up with 12 and 13 questions for the buyer and seller survey respectively that were used for measuring our variables of interest.

Table 5.2. Summary statistics of the variables included in the analysis

\begin{tabular}{|c|c|c|}
\hline Variable & $\begin{array}{l}\text { Description; Question \# in } \\
\text { survey, Appendix 5.b and } \\
\text { Appendix 5.c }\end{array}$ & Rationale \\
\hline \multicolumn{3}{|c|}{ Buyer and seller variables } \\
\hline $\begin{array}{l}\text { Fear towards } \\
\text { flooding }\end{array}$ & $\begin{array}{l}\text { Combined score on questions that } \\
\text { measure fear towards flooding; } Q \\
\text { II. } 2 \text { in A.5.b and A.5.C }\end{array}$ & $\begin{array}{l}\text { The affect heuristic and protection } \\
\text { motivation theory state that fear drives } \\
\text { people's perception of risk and can } \\
\text { motivate protective as well as non- } \\
\text { protective responses (Van Duinen et al. } \\
2015 \text { ) }\end{array}$ \\
\hline $\begin{array}{l}\text { Information and } \\
\text { learning about } \\
\text { flood risk }\end{array}$ & $\begin{array}{l}\text { Sources and ways to get informed } \\
\text { about flood risk (personal } \\
\text { experience, family or friends, } \\
\text { neighbours, FEMA flood maps, }\end{array}$ & $\begin{array}{l}\text { Various sources of information can } \\
\text { dynamically affect (positively and } \\
\text { negatively) people's risk perceptions, }\end{array}$ \\
\hline
\end{tabular}




\begin{tabular}{|c|c|c|}
\hline & $\begin{array}{l}\text { news media, or other sources); } Q \\
\text { VI.I A.5.b and A.5.c }\end{array}$ & $\begin{array}{l}\text { fear, and motivation to adopt protective } \\
\text { responses }\end{array}$ \\
\hline $\begin{array}{l}\text { Not learning } \\
\text { about flood risk }\end{array}$ & $\begin{array}{l}\text { Indicates whether people are } \\
\text { informed about flood risk at all or } \\
\text { not; Q VI.I A.5.b and A.5.c }\end{array}$ & $\begin{array}{l}\text { Being informed about hazard risks is } \\
\text { essential in order to start considering the } \\
\text { benefits of risk management strategies }\end{array}$ \\
\hline $\begin{array}{l}\text { Experienced } \\
\text { flooding in town }\end{array}$ & $\begin{array}{l}\text { Indicates whether people have } \\
\text { experienced a major flooding event } \\
\text { in their town; Q V.I A.5.b and } \\
\text { A.5.c }\end{array}$ & $\begin{array}{l}\text { Experience is considered an important } \\
\text { source of information on which people } \\
\text { base their perceptions and decisions } \\
\text { (Bubeck et al. 20I3) }\end{array}$ \\
\hline $\begin{array}{l}\text { Damage from } \\
\text { flooding }\end{array}$ & $\begin{array}{l}\text { Indicates whether people have } \\
\text { experienced any damages from a } \\
\text { flooding event; Q V.4 A.5.b and } \\
\text { A.5.c }\end{array}$ & $\begin{array}{l}\text { Damage, as an indicator of bad } \\
\text { experience, can enhance people's fear } \\
\text { towards the risk according to the affect } \\
\text { heuristic theory (Van Duinen et al. 2015) }\end{array}$ \\
\hline $\begin{array}{l}\text { Probability of } \\
\text { flooding }\end{array}$ & $\begin{array}{l}\text { Indicates whether a property } \\
\text { (bought or sold) is in a flood zone, } \\
\text { and in which flood zone, to the } \\
\text { knowledge of the respondents; Q } \\
\text { IV.8 A.5.b and Q IV.I2 A.5.c }\end{array}$ & $\begin{array}{l}\text { In expected utility theory the objective } \\
\text { probability of a flood forms the base of } \\
\text { people's perceived probability and hence } \\
\text { their risk perception }\end{array}$ \\
\hline $\begin{array}{l}\text { Perceived } \\
\text { likelihood of } \\
\text { damage }\end{array}$ & $\begin{array}{l}\text { Indicates the perceived likelihood } \\
\text { that their property will get damages } \\
\text { from flooding; Q II.I A.5.b and } \\
\text { A.5.c }\end{array}$ & $\begin{array}{l}\text { This variable can elicit the function by } \\
\text { which people value the objective } \\
\text { probability of flood under prospect theory } \\
\text { (Lindel and Hwang 2008, Van Duinen et } \\
\text { al. 20I5, Trumbo et al. 2014) }\end{array}$ \\
\hline \multicolumn{3}{|l|}{ Buyer variables } \\
\hline $\begin{array}{l}\text { Take flood risk } \\
\text { into account in } \\
\text { the search for a } \\
\text { home }\end{array}$ & $\begin{array}{l}\text { Buyers who have thought about } \\
\text { flood zones when they started } \\
\text { looking for a property; Q IV.9 A.5.b }\end{array}$ & $\begin{array}{l}\text { Before even considering how to deal with } \\
\text { the risk of flooding, buyers first need to } \\
\text { take the risk into consideration }\end{array}$ \\
\hline $\begin{array}{l}\text { Avoid flood } \\
\text { zones }\end{array}$ & $\begin{array}{l}\text { Average score on the statement 'If } \\
\text { a property was in a ... flood zone } \\
\text { (or flooded before), I would never } \\
\text { buy this property'; Q II.2 A.5.b }\end{array}$ & $\begin{array}{l}\text { Dependent variable to measure whether } \\
\text { risk perception and fear actually lead to a } \\
\text { protective response in the form of risk } \\
\text { avoidance }\end{array}$ \\
\hline $\begin{array}{l}\text { Willingness to } \\
\text { pay (\%) }\end{array}$ & $\begin{array}{l}\text { Buyers' bid price or transaction } \\
\text { price relative to the listing price } \\
\text { when buyers placed their first offer; } \\
\text { Q IV.I and IV.2 A.5.b }\end{array}$ & $\begin{array}{l}\text { In expected utility theory the expected } \\
\text { costs of the risk should equal a reduction } \\
\text { in the willingness to pay }\end{array}$ \\
\hline $\begin{array}{l}\text { Get flood } \\
\text { insurance }\end{array}$ & $\begin{array}{l}\text { Preparedness to get flood } \\
\text { insurance on the new home; Q III.I } \\
\text { A.5.b }\end{array}$ & $\begin{array}{l}\text { Dependent variable to measure whether } \\
\text { the perceived risk leads to a protective } \\
\text { response in the form of flood insurance } \\
\text { (Van Duinen et al. 20I5, Bubeck et al. } \\
2013 \text { ) }\end{array}$ \\
\hline $\begin{array}{l}\text { Perceived costs } \\
\text { of mitigation } \\
\text { measure }\end{array}$ & $\begin{array}{l}\text { Perceived costs of various risk } \\
\text { mitigation measures (get flood } \\
\text { insurance, install flood vents, selling } \\
\text { the house and move out of the } \\
\text { flood zone, elevating the property } \\
\text { and abandon the lowest flood); Q } \\
\text { III.3 A.5.b }\end{array}$ & $\begin{array}{l}\text { Protection motivation theory variable that } \\
\text { is part of people's cost-effectiveness } \\
\text { evaluation of the mentioned risk } \\
\text { management strategies (Van Duinen et al. } \\
2015 \text {, Bubeck et al. 20I3) }\end{array}$ \\
\hline
\end{tabular}




\begin{tabular}{|c|c|c|}
\hline $\begin{array}{l}\text { Perceived } \\
\text { effectiveness of } \\
\text { mitigation } \\
\text { measure }\end{array}$ & $\begin{array}{l}\text { Perceived effectiveness of various } \\
\text { risk mitigation measures (get flood } \\
\text { insurance, install flood vents, selling } \\
\text { the house and move out of the } \\
\text { flood zone, elevating the property } \\
\text { and abandon the lowest floor); Q } \\
\text { III.2 A.5.b }\end{array}$ & $\begin{array}{l}\text { Protection motivation theory variable that } \\
\text { is part of people's cost-effectiveness } \\
\text { evaluation of the mentioned risk } \\
\text { management strategies (Van Duinen et al. } \\
20 \text { I5, Bubeck et al. 20I3) }\end{array}$ \\
\hline $\begin{array}{l}\text { Adopt } \\
\text { protective } \\
\text { measures }\end{array}$ & $\begin{array}{l}\text { Preparedness to adopt any } \\
\text { protective measures to reduce } \\
\text { damage from flooding (installing } \\
\text { flood vents, elevating the property } \\
\text { or abandoning the lowest floor); Q } \\
\text { III.I A.5.b }\end{array}$ & $\begin{array}{l}\text { Dependent variable to measure whether } \\
\text { the perceived risk leads to a protective } \\
\text { response in the form of structural } \\
\text { modifications to the property (Van } \\
\text { Duinen et al. 2015, Bubeck et al. 2013) }\end{array}$ \\
\hline \multicolumn{3}{|l|}{ Seller variables } \\
\hline $\begin{array}{l}\text { I00-year flood } \\
\text { zone AND } \\
\text { experienced } \\
\text { flooding in town }\end{array}$ & $\begin{array}{l}\text { Indicates sellers of property in the } \\
\text { I00-year flood zone who have } \\
\text { experienced a flood in their town; } \\
\text { Q IV.I } 2 \text { and Q V.I A.5.c }\end{array}$ & $\begin{array}{l}\text { Floods have a high impact in the } 100 \text {-year } \\
\text { flood zones, which will negatively impact } \\
\text { the value of a property }\end{array}$ \\
\hline $\begin{array}{l}\log (\$) \\
\text { willingness to } \\
\text { accept for } \\
\text { property }\end{array}$ & $\begin{array}{l}\text { Transaction price of recently sold } \\
\text { property or current list price of } \\
\text { property still on the market on a } \\
\text { log scale; Q IV.3 A.5.c }\end{array}$ & $\begin{array}{l}\text { In expected utility theory the expected } \\
\text { costs of the risk should be discounted by } \\
\text { sellers in the form of a willingness to } \\
\text { accept }\end{array}$ \\
\hline $\begin{array}{l}\text { Moving out of } \\
\text { the flood zone }\end{array}$ & $\begin{array}{l}\text { Sellers who have moved away from } \\
\text { the flood zone after selling their } \\
\text { property; Q III.I A.5.c }\end{array}$ & $\begin{array}{l}\text { Dependent variable to measure whether } \\
\text { risk perception and fear lead to future } \\
\text { risk avoidance }\end{array}$ \\
\hline $\begin{array}{l}\text { Moving out to } \\
\text { evade the } \\
\text { hazard }\end{array}$ & $\begin{array}{l}\text { Sellers who explicitly mentioned } \\
\text { that the risk of natural hazards was } \\
\text { a reason to sell their property; Q } \\
\text { IV.I A.5.c }\end{array}$ & $\begin{array}{l}\text { Moving away from the flood zone may be } \\
\text { directly related to risk avoidance } \\
\text { behaviour }\end{array}$ \\
\hline $\begin{array}{l}\text { Costs of flood } \\
\text { insurance }\end{array}$ & $\begin{array}{l}\text { Perceived costs of flood insurance } \\
\text { only for those households that had } \\
\text { flood insurance on the property } \\
\text { they sold. The value ranges I-5, or } \\
0 \text { otherwise; Q III.3 A.5.C }\end{array}$ & $\begin{array}{l}\text { Moving away from the flood zone may be } \\
\text { related to the costs of mandatory flood } \\
\text { insurance rather than risk perception }\end{array}$ \\
\hline $\begin{array}{l}\text { Costs of } \\
\text { moving }\end{array}$ & $\begin{array}{l}\text { Perceived costs of the measure to } \\
\text { sell the property and move away } \\
\text { from the flood zone; Q III.3 A.5.C }\end{array}$ & $\begin{array}{l}\text { The perceived costs of moving, falling } \\
\text { under protection motivation theory, may } \\
\text { inhibit people to sell and move away from } \\
\text { the flood zone }\end{array}$ \\
\hline $\begin{array}{l}\text { Perceived } \\
\text { effectiveness of } \\
\text { moving to } \\
\text { reduce risk }\end{array}$ & $\begin{array}{l}\text { Perceived effectiveness of the } \\
\text { measure to sell the property and } \\
\text { move away from the flood zone as } \\
\text { a way to minimize future damages } \\
\text { from flooding; Q III.2 A.5.c }\end{array}$ & $\begin{array}{l}\text { Falling under protection motivation } \\
\text { theory, the perceived effectiveness of } \\
\text { reducing the hazard exposure by moving } \\
\text { away from the flood zone may motivate } \\
\text { people to relocate (Van Duinen et al. } \\
2015 \text {, Bubeck et al. 20I3) }\end{array}$ \\
\hline
\end{tabular}

We tried to minimize order effects in the survey by asking first about flood risk perceptions and mitigation, and later the actual decisions and experiences. Yet, the 
first questions may bias the response to questions about the role that flood risk has played in the purchase or selling decision. Hence, respondents could potentially overstate the importance of flood risk in their decisions, in particular those that have a high risk perception. This may strengthen the signal in our data on the role of flood risk perception in the decisions to adopt protective responses.

We examine 4 behavioural responses of buyers and 3 responses of sellers that relate directly to flood risk and how it affects the market value of properties in the flood zone. For buyers: I) considering flood zones when looking for a property (Part IV, question 9 in Appendix 5.b), 2) avoiding a property in the flood zones, 3) getting flood insurance on the new property (Part III, question I in Appendix 5.b), and 4) paying less for properties that are located in a flood zone. For sellers: I) moving out of the flood zone (Part III, question I in Appendix 5.c), 2) moving primarily to evade the hazard of flooding (Part IV, question I in Appendix 5.c), and 3) asking for a lower price if the property is within a flood zone.

The tendency to avoid property in the flood zone is measured by combining three questions on a Mokken scale ( $\alpha=0.73$ ): I) not buying property that has been flooded before (easy), 2) not buying property in the flood zone with an annual 1:100 chance of flooding (intermediate) and 3 ) not buying property in the flood zone with an annual 1:500 chance of flooding (difficult). The scores are normalised based on the mean score of all respondents on each of the three items, resulting in the average score per respondent.

$X_{i}=\sum_{j=1}^{3}\left(\frac{x_{i j}}{\sum_{i=1}^{N} x_{i j} / N}\right)$

In Eq. 5.I $N$ is the number of survey respondents and $x_{i j}$ indicates the score of respondent $\mathrm{i}$ on item $\mathrm{j}$.

Buyers' willingness to pay for properties in the flood zones is measured by looking at their bid price or purchase price relative to the listing price (in \%) at the time of bidding, and can be negative or positive depending on whether buyers bid under or over ask price respectively. We ask buyers to give both values because we know that buyers typically anchor their bids on the sellers' ask price. Therefore there is no need to control for various housing characteristics which is common practice in hedonic studies that measure the marginal contribution of various housing attributes to the sales price of properties on the market, such as a discount for properties that are prone to flooding. We compare the offers for properties outside a flood zone with those inside a flood zone. We ask the respondents to inform us whether the property they bought (or are considering buying) is located inside any flood zone, 
and about the annual probability of flooding for these flood zones, regardless of whether this information is correct or known to the buyer. Primarily because our interest is in homeowners' responses to their perception of flood risk rather than objective information of flood risk.

Similarly, we measure if location in a flood zone affects sellers' ask price for properties. This requires more information about the properties to control for various structural and spatial characteristics, which is typical in any hedonic study of housing prices (Rosen 1974). We take into account square footage, whether a property is located on the waterfront, presence of a garage and Zillow Home Value Index (Zillow 2018). The latter is a spatial measure of housing values that controls for the market where the property is sold. For example: the median value of a property in Miami (FL) is twice as much as the mean value of a property in Jacksonville (FL). Hedonic studies commonly use a lot more variables that matter in housing prices, but these studies often have a lot more data available both in number of transactions and in number of housing attributes. We choose to limit the number of variables to prevent overloading the respondents with detailed questions on housing attributes, and to prevent over-parameterising our model (de Koning et al. 2016).

Additionally, we are interested in homeowners' perceived likelihood that flooding can cause damage to their property. This is only partly affected by the objective knowledge of being located in a flood zone, and may be much more important in governing decisions in the market. We measure the perceived likelihood of damages on a qualitative scale: "How likely is it that your house will get damage from flooding?" - answered on a 4 point scale with end labels I ="very unlikely" and 4="very likely". Despite numerous attempts to measure perceived probabilities on a quantitative scale (Botzen et al. 2009, Katapodi et al. 2004, Weinstein 2000) we decide that it is better to avoid this line of questioning for the following reasons: people find it difficult to understand probability statistics, people find it hard to express their perceived probabilities, the answers that people give are anchored on what the options are, and qualitative assessments are often better predictors than quantitative ones (Weinstein et al. 2007).

Finally, we measure people's fear towards flooding, which is treated as separate risk perception variable in the psychometric paradigm (Slovic et al. 2005) and protection motivation theory (Grothmann and Reusswig 2006). This variable combines the scores of two questions that measure fear $(\alpha=0.77)$ (Part II, question $2 g$ and $2 \mathrm{~h}$ in Appendix 5.b), and is calculated in the same way as Eq. 5.I. 
Apart from the behavioural responses that relate to location choice and willingness to pay/willingness to accept for properties at risk, we enquire about a few alternatives that reduce households' flood risk in case they live in a flood zone. We select a few options suggested by the Federal Emergency Management Agency (FEMA) that have a high potential in reducing damage from flooding (FEMA 20I5), namely: installing flood vents/openings (routes for the water to drain), elevating the property, and abandoning the lowest floor. We measure all coping appraisal variables of protection motivation theory related to these options: protective response efficacy (i.e. how effective do you think this measure is in reducing your risk?), perceived self-efficacy (i.e. how easy or difficult do you think it is to adopt this measure in your home?) and perceived response costs. The same is measured for the options to get flood insurance and to sell the property and move away from the flood zone.

\subsubsection{Statistical analyses - Bayesian statistics}

Above mentioned variables on the risk perception and mitigation behaviour and their relation are selected using a number of explanatory variables that we consider relevant in affecting risk perceptions and mitigation behaviour. Table 5.3 provides an overview of the dependent variables (first column) and the variables that are used to predict them (second column). Next, we run a stepwise regression with backward selection based on the Akaike information criterion in order to select those variables that are significant in explaining the dependent variable. We also check the signs of the coefficients to evaluate whether the outcome of the stepwise regression analysis makes sense.

Table 5.3. List of explanatory variables and dependent variables included in the stepwise regression.

\begin{tabular}{ll}
\hline $\begin{array}{l}\text { Dependent } \\
\text { variables }\end{array}$ & Explanatory variables considered \\
\hline $\begin{array}{l}\text { Fear towards } \\
\text { flooding }\end{array}$ & $\begin{array}{l}\text { Learning about floods through FEMA, learning about floods through } \\
\text { neighbours, learning about floods through family and friends, learning about } \\
\text { floods through news media, not learning about flood risk, damage from } \\
\text { flooding, experienced flooding in town, probability of flooding, perceived } \\
\text { likelihood of damage }\end{array}$ \\
$\begin{array}{l}\text { Install flood vents, } \\
\text { elevate the property, } \\
\text { abandon the lowest } \\
\text { floor }\end{array}$ & $\begin{array}{l}\text { Peasure, perceived self-efficacy of mitigation measure, fear towards flooding, } \\
\text { pvoid flood zone }\end{array}$ \\
$\begin{array}{l}\text { Perceived likelihood of damage } \\
\text { efficacy of moving, fear towards flooding, perceived likelihood of damage, } \\
\text { probability of flooding }\end{array}$
\end{tabular}


Get flood insurance Perceived costs of flood insurance, perceived effectiveness of flood insurance, perceived self-efficacy of flood insurance, fear towards flooding, perceived likelihood of damage, take flood risk into account in the search for a home

Take flood risk into Learning about floods through neighbours, learning about floods through account in the family and friends, learning about floods through news media, not learning search for a home about flood risk, damage from flooding, experienced flooding in town Perceived likelihood Learning about floods through neighbours, learning about floods through of damage (buyer) news media, not learning about flood risk, damage from flooding, experienced flooding in town, probability of flooding $\log (\$)$ willingness to Square footage (log), house on the waterfront, house with a garage, Zillow home value index, 100-year flood zone AND experienced flooding in town, 500-year flood zone AND experienced flooding in town, damage from flooding, experienced flooding in town, 100-year flood zone dummy, 500year flood zone dummy, perceived likelihood of damage

Moving out of the flood zone Perceived costs of moving, perceived effectiveness of moving, get flood insurance, get flood insurance AND perceived costs of flood insurance, fear towards flooding, perceived likelihood of damage, damage from flooding

Moving out to evade the hazard Perceived costs of moving, perceived effectiveness of moving, fear towards flooding, perceived likelihood of damage, damage from flooding Perceived likelihood Learning about floods through FEMA, learning about floods through of damage (seller) neighbours, learning about floods through family and friends, learning about floods through news media, not learning about flood risk, perceived likelihood of damage, fear towards flooding, damage from flooding, experienced flooding in town, probability of flooding

The models with the lowest number of informative explanatory variables are used to construct Fig. 5.I and 5.2. We use the Bayesian approach to support the evidence for the causal relationships between the variables depicted in Fig. 5.1 and 5.2 (Howson and Urbach 2006). We apply Bayesian statistics as an addition to the stepwise regressions that yield $\mathrm{p}$-values of the coefficients. Bayesian statistics is used to assess the likelihood of a certain hypothesis given the available data, $\mathrm{p}(\mathrm{H} \mid$ data $)$, whereas the frequency statistics yield the likelihood of the data given a certain hypothesis, $\mathrm{p}($ data| $\mathrm{H})$. More specifically, $\mathrm{p}$-values used in frequency statistics indicate the likelihood of the data given the null-hypothesis (i.e. there is no causal relationship or correlation between the tested variables), $\mathrm{p}\left(\right.$ data| $\left.\mid \mathrm{H}_{0}\right)$, and can subsequently lead to the rejection of this null-hypothesis. The problem with this frequentist approach is that it can result in type-I and type-Il errors, i.e. false rejection of the nullhypothesis or failure to reject the false null-hypothesis. Contrastingly, the benefit of using the Bayesian approach is that it allows for simultaneously assessing the likelihood of a set of alternative hypotheses, including the null-hypothesis, based on the available data and a set of prior likelihoods of combinations of hypotheses and data. Instead of an arbitrary cut-off value (typically: $\alpha=0.05$ ) that leads to the 
rejection of a hypothesis, Bayesian statistics can yield a likelihood estimation that a certain hypothesis is true without having to falsify any alternative hypothesis. This hypothesis can for example be about the value of a certain parameter in a regression model. As Ellison (2004) phrases: “Bayes' Theorem provides an intuitively clear alternative method for estimating parameters and expressing the degree of confidence or uncertainty in those estimates. Bayesian methods allow for the explicit incorporation of as much or as little existing data or prior knowledge that is available, and provides a direct measure of the probability of one or more hypotheses of interest."

The Bayesian approach forces the researcher to be explicit in the prior assumptions about the hypotheses and the data, and specify the prior likelihood of one or more hypotheses. At this stage it can be decided a priori that certain hypotheses are more likely than others, which is not accounted for in frequency statistics. These priors can be derived from earlier studies, so that each new study adds knowledge to the previous one, which builds increasing evidence and confidence in supporting a particular hypothesis with every new study. The list of priors used for this study is summarised in Table A3 (Appendix 5.a). The priors for sellers' ask price and buyers' willingness to pay are derived from a meta-analysis of hedonic studies that assesses how flood risk affects the value of properties in the flood zones (Beltrán et al. 2018). The prior likelihood of the effect size is a normal distribution, which means that the effect size, a priori, is most likely the mean of all hedonic studies on flood risk and property values. For all other variables we use uninformative priors, i.e.: the true effect could be any value between a certain range with an equal likelihood for each value within this range, which is a uniform distribution between a minimum and a maximum.

For each relation between the variables we form a set of mutually exclusive hypotheses:

$\mathrm{H}_{0}$ : There is no effect between the independent and dependent variable.

$\mathrm{H}_{\mathrm{al}}$ : The independent variable has a negative effect on the dependent variable.

$\mathrm{H}_{\mathrm{a} 2}$ : The independent variable has a positive effect on the dependent variable.

These hypotheses meet two conditions: (I) They are mutually exclusive, i.e.: $\mathrm{p}\left(\mathrm{H}_{0} \mathrm{U}\right.$ $\left.\mathrm{H}_{\mathrm{a}} \cup \mathrm{H}_{\mathrm{a} 2}\right)=\mathrm{p}\left(\mathrm{H}_{0}\right)+\mathrm{p}\left(\mathrm{H}_{\mathrm{al}}\right)+\mathrm{p}\left(\mathrm{H}_{\mathrm{a} 2}\right)$. (2) They cover the full range of all possible outcomes, i.e.: $\mathrm{p}\left(\mathrm{H}_{0}\right)+\mathrm{p}\left(\mathrm{H}_{\mathrm{a} 1}\right)+\mathrm{p}\left(\mathrm{H}_{\mathrm{a} 2}\right)=\mathrm{I}$. Furthermore, we allow for a certain range of negligible effect sizes around $\mathrm{H}_{0}$, which increases the likelihood of $\mathrm{H}_{0}$, whereas it is nearly impossible to find an effect size that is exactly equal to 0 . We neglect effect sizes smaller than 0.10 times the standard deviation of the dependent variable, which 
falls in the range of $\mathrm{H}_{0}$. Any effect sizes larger than 0.10 times the standard deviation of the dependent variable fall in the range of $\mathrm{H}_{\mathrm{al}}$ or $\mathrm{H}_{\mathrm{a} 2}$. The posterior probabilities of $\mathrm{H}_{0}, \mathrm{H}_{\mathrm{a} 1}$ and $\mathrm{H}_{\mathrm{a} 2}$ are calculated as follows:

$$
\begin{aligned}
& p(H \mid \text { data })=\frac{p(H) * p(\text { data } \mid H)}{p(\text { data })}=\frac{p(H) * p(\text { data } \mid H)}{\int_{\min }^{\max } p(H) * p(\text { data } \mid H) d H} \\
& \int_{\min }^{\max } p(H) d H=1 \\
& p(H) \ni p\left(H_{0}\right), p\left(H_{a 1}\right), p\left(H_{a 2}\right)
\end{aligned}
$$

$\mathrm{P}(\mathrm{H})$ in Eq. 5.2, 5.3 and 5.4 is the function that describes the prior probability density of the effect size, which is a continuous probability function of all possible effect sizes, the sum of which equals to I (Eq. 5.3). For computational purposes we limit these to 10,001 discrete hypotheses between a predefined minimum and maximum. $\mathrm{p}($ data $\mid \mathrm{H})$ in Eq. 5.2 is the probability density of our data for each hypothesis (note that the specific case of $p\left(\right.$ data| $\left.\mathrm{H}_{0}\right)$ in Eq. 5.2 is the $\mathrm{p}$-value yielded from t-tests, ANOVAs and regression analyses), $\mathrm{P}$ (data) is the weighed probability of the data for all hypotheses, and $\mathrm{p}(\mathrm{H} \mid$ data $)$ is the posterior probability density function of the hypotheses. The latter is used to calculate the likelihood of each of the hypotheses $\mathrm{H}_{0}, \mathrm{H}_{\mathrm{a}}$ and $\mathrm{H}_{\mathrm{a}}$, by assessing which hypothesis $\mathrm{H}$ belongs to which subset in Eq. $5.4\left(\mathrm{H}_{0}, \mathrm{H}_{\mathrm{a}}\right.$ or $\left.\mathrm{H}_{\mathrm{a} 2}\right)$ and summing the probabilities $\mathrm{p}(\mathrm{H})$ for each subset.

Besides the mean and the range of possible effect sizes, we formed some additional underlying assumptions about the data. First, we assume a priori that the probabilities of the dependent variables in the data follow either a normal or binomial distribution. Second, we assume that the impact on the dependent variable is always linear. We acknowledge that this may not always be the case, but it is sufficient for the purpose of highlighting either a positive or negative relationship between the independent and dependent variable.

\subsection{Results}

\subsection{Cognitive and affective drivers of households' responses to flood risk}

In our study we find that fear towards flooding, damages from flooding and perceived likelihood of damage are the three main factors that drive households' responses to flood risk. For buyers these responses are: considering flood zones when looking for a property, avoiding property in the flood zones, and getting flood insurance on the 
new property. For sellers the responses are: moving out of the flood zone ${ }^{23}$, and accepting a lower price for their property if it is located in a flood zone. Fig. 5.I and 5.2 show all the relevant relations between risk perception variables, experience, information and learning and their effect on the decisions to either self-protect against flooding or to evade the hazard. The strength of the effect is highlighted by the thickness of the lines, the darkness of the lines indicates the strength of the evidence for the hypothesised effect $\left(\mathrm{H}_{\mathrm{a}}\right)$, and colours indicate whether the response was positive or negative $\left(\mathrm{H}_{\mathrm{al}}\right.$ or $\left.\mathrm{H}_{\mathrm{a} 2}\right)$. Fear, driven by (lack of) information and experience, is the main reason for buyers to avoid the flood zone (Fig. 5.I), with an effect size of 0.97 standard deviation ${ }^{24}$. While the adoption of other protective responses (in particular flood insurance) is primarily driven by the perceived likelihood of damage (effect size of 0.78 standard deviation in the case of getting flood insurance), and to a lesser extent by other cognitive decision processes and variables included in protection motivation theory. On the seller's side we see that the decision to move out of the flood zone is a combination of affective as well as cognitive decision processes, though this includes people that sell their house for different reasons than flood risk alone. People who sell their house explicitly to avoid the risk of natural hazards do so for affective reasons: fear and experience with flood damage (effect sizes of 0.37 and 0.90 standard deviation respectively). Experience with flooding in town and damage to property are the only factors that reduced sellers' ask price for properties in the 1:100 annual flood zone. The probability of flooding has a positive effect on the ask price, which may be counter-intuitive, but is actually a very common finding because properties at high risk of flooding are often associated with high environmental amenities such as living close to the beach or living close to water (Bin et al. 2008).

\footnotetext{
${ }^{23}$ A certain group of people were living in the flood zone and decided to sell their house for various reasons, while they were looking for a new home outside the flood zone. A subgroup of these people decided to abandon the flood zone explicitly because of the risk of natural hazards.

24 The effect size depends on the coefficient of the linear regression, the range (min-max) of the independent variable and the standard deviation of the dependent variable.
} 


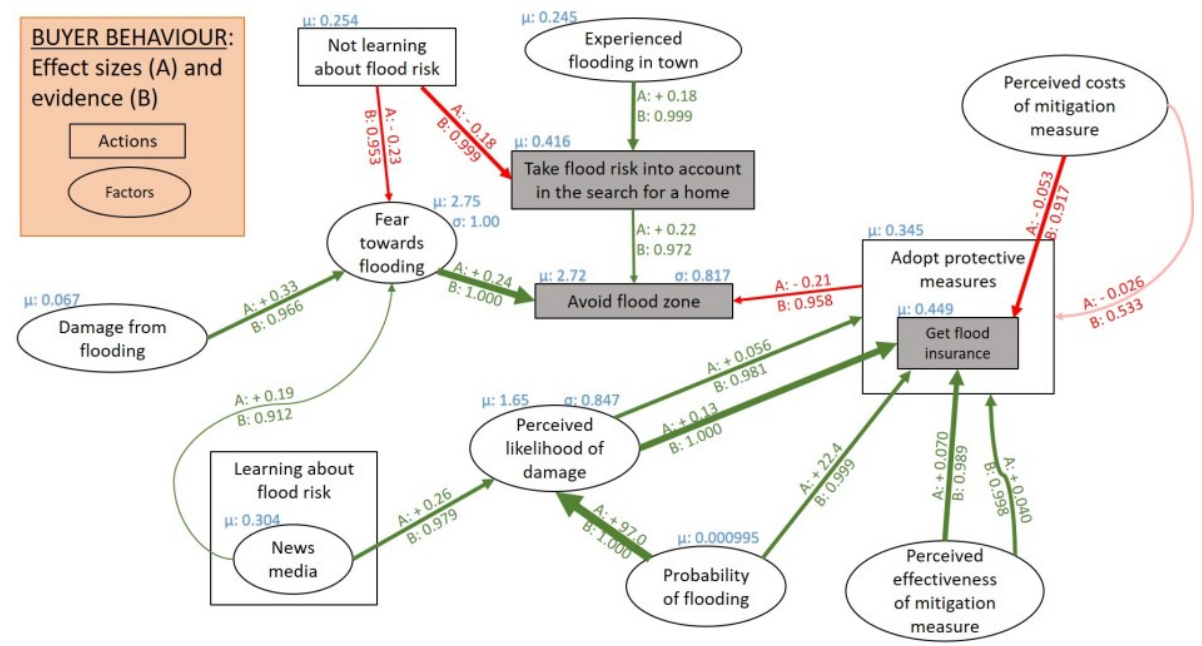

Figure 5. I. Impacts and effect sizes (A) of factors that govern buyers' responses to flood risk. B highlights the Bayesian posterior likelihood of the effect. Red indicates negative effects and green the positive. Lighter arrows indicate weaker evidence for the effect. We added the mean value of each variable $(\mu)$ and, if applicable, the standard deviation $(\sigma)$ that allow for a more meaningful interpretation of the effect size. Thicker lines indicate a stronger effect.

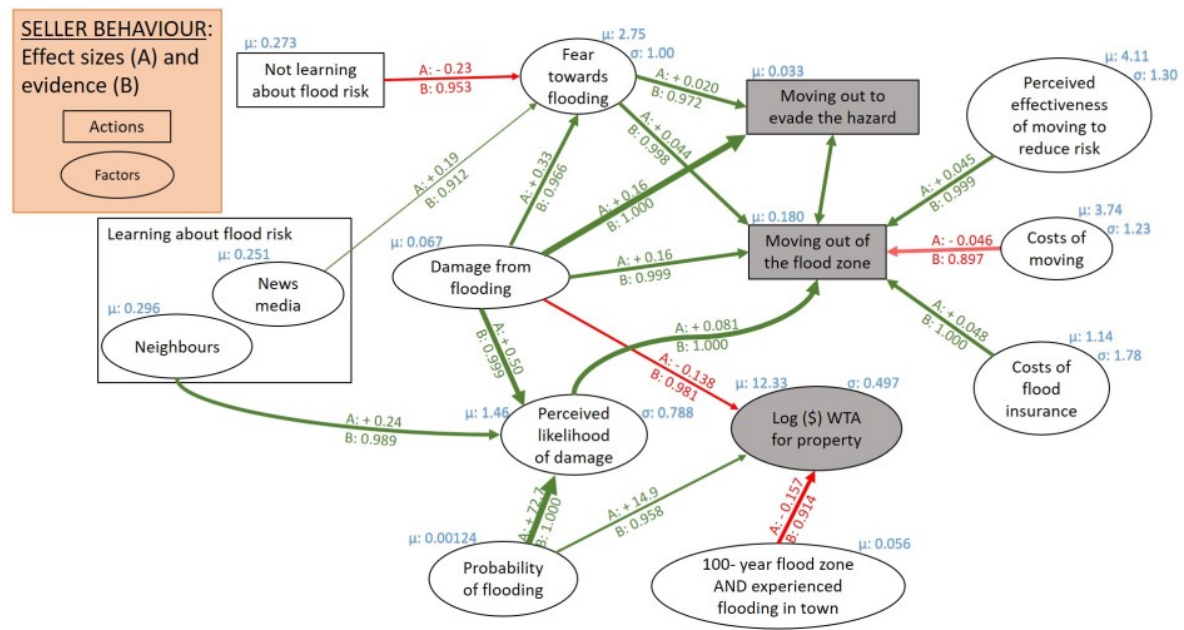

Figure 5.2. Impacts and effect sizes (A) of factors that govern sellers' responses to flood risk. B highlights the Bayesian posterior likelihood of the effect. Red indicates negative effects and green the positive. Lighter arrows indicate weaker evidence for the effect. We added the mean value of each variable $(\mu)$ and, if applicable, the standard deviation $(\sigma)$ that allow for a more meaningful interpretation of the effect size. Thicker lines indicate a stronger effect. The main dependent variables are highlighted in grey. The box 'moving out of the 
flood zone' indicates people that sell their property for all sorts of reasons, and choose to live outside the flood zone after the move. The box 'moving out to evade the hazard' is a subset of the former group that do so explicitly to escape the hazard of flooding.

In Fig. 5.2 we exclude sellers' protective measures against flooding, even though we find the same factors to be relevant in their decisions to self-protect against flooding as in the buyer survey. Yet, for sellers the adoption of protective measures against flooding has no further consequences for their decision to sell their property, which means moving out of the flood zone.

These results show that affective decision making drives buyers to evade the flood zone and sellers to abandon their homes at risk of flooding. For buyers the effect of fear is almost four times stronger than the cognitive processes of thinking about flood risk when searching for a property and the adoption of measures to reduce the risk of flooding. Also for sellers, fear and experience with damage are the only drivers that makes them abandon the flood zone by selling their property. Damage from flooding is particularly impactful, making it almost five times more likely for homeowners to abandon the flood zone by selling their property. An increase in fear could also easily double this likelihood. The decisions to adopt protective measures against flooding are more objective, and are influenced by the perceived likelihood of damage, perceived control efficacy and perceived control costs. For example, the preparedness to get flood insurance is positively affected by perceived control efficacy of flood insurance, the perceived costs of flood insurance and the actual probability of flooding. The latter is most likely caused by the fact that flood insurance in the U.S. is mandatory for properties in the 1:100 annual flood zone that are financed by a federally insured mortgage.

Sellers that move out of the flood zone do so for various reasons, ranging from cognitive appraisals of costs, risks and effects to affective responses such as experience and fear. It should be noted that sellers who move out of the flood zone do not always do so explicitly because of the risk. They may initially have different reasons to sell their house, but in the process they also decide to evade the flood zone in their future home. The choice could also be to avoid the costs of flood insurance, and thus made independent of flood risk. Only a small proportion of respondents (3.3\%) explicitly mention that their reason to sell is to avoid the risk of flooding.

\subsubsection{Which information drives perceptions}

News media and personal experience are the primary information sources that affect feelings of fear for both sellers and buyers. Fear is positively affected by experience 
with damage from flooding and to some extent also by news media, though this effect is rather small (+/- 0.19 standard deviation). The Bayesian posterior likelihoods of these positive effects are $96.6 \%$ and $91.2 \%$ respectively based on the survey data (Fig. 5.1 \& 5.2), and the effect of damage on fear is 1.7 times larger than media on fear. People who are not informed about flood risk have a lower fear towards flooding (0.23 standard deviation), implying that a lack of information about a hazard reduces fear towards it. Consequently, these buyers do not take flood zones into account in their choice where to buy property. Buyers that ever experienced a flooding event in their town are more likely to take flood zones into account. It does not affect their level of fear, likely because not everyone who experiences flooding in their town regards it as a dreadful experience. Furthermore, taking the flood zones into account in the choice where to buy property is somewhat related to the tendency to evade the flood zones altogether, but it is not a one-on-one correlation. We did not ask for more details about what their considerations are about the flood zones, apart from avoiding them. Perhaps these are also related to other protective measures, or even to the positive aspects of living in flood zones (e.g. living close to the beach or on the water front). We do not take these into account in our analysis.

The perceived likelihood of damage is to a large extent governed by objective information on the probability of flooding, i.e. the knowledge that a house is located in the flood zone. Interestingly, information from neighbours affected the perceived likelihood of damage for sellers, and buyers' perceived likelihood of damage was influenced by news media. Neighbours' experience with flooding as well as personal damages has a positive effect on sellers' perceived likelihood of damage, most likely because they have already been living in the area for a long time. While buyers, who are the newcomers to the area, are influenced by information from news media and not by their own experience or experience from neighbours.

\subsubsection{Effects on property values in hazard areas}

Given that flood risk influences the decisions that buyers make in the market in terms of evading the flood zones, it is expected that sellers in the flood zones anticipate on this by lowering their ask price. Similarly, we expect that buyers offer a lower price for a property in the flood zone. We do find such an effect for sellers who have experienced a flood, in particular those that have had damage from flooding, but not for buyers. Buyers' willingness to pay is unaffected by flood risk, probably because the risk-sensitive buyers are already sorted out by avoiding the flood zones. On average, sellers in the flood zones demand a higher price for their property, unless their town is hit by a major flooding event or their property is damaged by flooding. A higher price for properties in the flood zones is often found in many hedonic pricing 
studies that valuate the risk of flooding (e.g. Bin and Kruse 2006, Bin et al. 2008, Hallstrom and Smith 2005), and is likely caused by positive amenities associated with flood zones. It is likely that sellers in the I:100 annual flood zone anticipate on a drop in demand after a major flooding event, causing them to drop their price. However, the evidence for this effect is only a little over $91 \%$, which leaves a $9 \%$ probability of a type-I error (false positive). The evidence that direct damages from flooding negatively affect sales prices is higher: $98 \%$.

\subsection{Discussion and conclusions}

This study explores several climate adaptation strategies of homeowners, that are driven by various behavioural triggers. We conducted a survey in January 2018 among households in hazard-prone areas in eight coastal states in the U.S., of which some have recently experienced a major flooding in their town. The survey results are analysed with Bayesian statistics to build on evidence of previous studies. We examine what behavioural mechanisms drive the trade-off between flood damage mitigation and relocation separately among property buyers and sellers, who shape demand for locations - and consequently future urbanization patterns - in floodprone cities.

\subsection{To leave or not to leave? Evasion versus adaptation to flood risk}

Flood risk is already the costliest natural hazard of all, and the impacts are only expected to increase in frequency and severity with climate change. In addition, the severity of flood damage is boosted by an increase in population and capital at stake. This means that flood damage mitigation measures will become more costly and decreasingly effective. Flood insurance in particular will become increasingly costly, both for policy holders as well as for insurance companies in case of a flooding event. Therefore, a complimentary strategy that contributes to societal resilience against ever-growing flood risks is to retreat from hazardous areas (Dronkers et al. 1990). The retreat can take a form of a governmental planned policy or be triggered from the bottom up by individuals' motivation to move. We assessed to what extent households are prepared to protect themselves against flooding by taking precautionary action, as well as by their choice whether to live in a flood-zone. The former has been studied well in numerous surveys, in particular to study what drives homeowners' preparedness to self-protect against flooding (Osberhaus 2017, Terpstra 20I I, Zaalberg et al. 2009), while the latter has received considerably less attention. Our survey assesses to what extent people who live in the flood zone are 
prepared to move away from the hazard, and potential newcomers are evading the risks of living in a flood zone. In particular, we elicit the psychological and cognitive mechanisms that drive these decisions, and assess how sensitively people respond to information from various sources, including their own experience with flooding events. We see that fear and experience with flooding can be important triggers for homeowners to retreat from hazard areas. This implies that bottom-up forces behind retreat (I) are highly sensitive to information that provoke people's feelings of fear inducing volatile responses to flood risk, and (2) rely on hazard events to trigger a protective action, which ideally would take place well before these events occur.

\subsubsection{Behavioural drivers of location choice and mitigation measures}

The risk perception factors that drive behavioural responses to flood risk are subject to change, driven by new information, personal experience and learning from others. In our study we find one key difference between evading and protecting against flood risk in the mechanisms that drive these behaviours. Abandoning and avoiding the flood zones are mostly driven by bad experiences and fear - buyers' fear has almost twice as much impact as other variables combined on the tendency to avoid the flood zones and sellers' decision to abandon the hazard zones is exclusively affected by experience with damage and fear - which fits the psychometric paradigm of affective decision making well (Slovic et al. 2005). The adoption of flood damage mitigation measures are governed by more cognitive decision processes that are incorporated in protection motivation theory (Grothmann and Reusswig 2006). Protection motivation theory is a comprehensive framework that explains well how people adapt to the increasing risk of flooding, and has a strong empirical basis. It has been studied well how risk and coping appraisals, as well as experience with flooding affect households' preparedness to take precautionary measures against flooding (Osberhaus 2017, Poussin et al. 2014, Terpstra 201I, Zaalberg et al. 2009). Given the previous work that has been done, we restrain from running an extensive analysis on all the variables that drive flood risk damage mitigation measures. Our results are consistent with the findings that perceived probability of damage and perceived costs and effectiveness of flood damage mitigation measures motivate the adoption of precautionary measures against flood damage. However, when it comes down to more radical measures to reduce flood risk, i.e. evading flood zones or selling one's flood-prone home, we see that the behavioural drivers shift from risk and response appraisal to fear. The latter is expected to be particularly relevant when hazard events occur more frequently and when more people experience flooding first-hand. 
Our primary concern is the different decision making processes between location choice and adopting flood damage mitigation measures, i.e.: evading flood zones and protecting against flood damage in a flood-prone location. Furthermore, we tested if there is any interaction between the two. Results indicate that fear and bad experience (severe damage from flooding) drive people away from hazard areas regardless whether they have taken damage mitigation measures to reduce their personal damage from flooding. Buyers' first decision is whether or not to evade flood zones in their search for a new home. The choice how to protect themselves against flooding is only a secondary decision. It appears that buyers' preparedness to adopt protective measures against flooding has a slight negative impact on the tendency to avoid flood zones, but the causality could be reversed - buyers that avoid the flood zones do not need to adopt any further protective measures against flooding. Therefore, we conclude that the choice whether or not to live in the flood zone is made separate from the choice to adopt flood damage mitigation measures.

\subsubsection{Existing patterns in individual cognitive mechanisms may drive regime shifts in the future.}

These finds raise a question on whether these cognitive mechanisms may cause any structural changes in urbanization in climate-sensitive areas in the long run, attenuating or amplifying resilience of these areas. In this study we find a few trends as a result of flooding events: I) people who experienced damage have an increased level of fear towards flooding, which drives both buyers and sellers away from flood zones, 2) fear is to a small extent also affected by media coverage of flooding events, 3) people who had damage from flooding are more likely to move out of the flood zone, regardless of any measures they took to self-protect against flooding, 4) sellers who had damage from flooding, as well as sellers in the $1: 100$ annual flood zone who experienced flooding in their town will demand a lower price for their property. These four mechanisms contribute to a drop in demand and an increased supply of (damaged) properties in the 1:100 annual flood zone, and subsequently a drop in property values in hazard zones in the wake of a flood. Acknowledging this, our findings suggest that bottom up forces behind aggregated demand and supply for housing in coastal urban areas may gradually change as probability and severity of floods intensify. The price trends in hazard-prone areas have been observed in previous difference-in-differences studies of property values before and after a flood event (Atreya et al. 2012, Bin and Landry 2013, Hallstrom and Smith 2005, Kousky 2010). Yet, none of them provide detailed insight in the bottom-up processes that drive these price changes, since they only look at transaction data. We highlight that homeowners' individual climate adaptation strategies may suddenly change when people experience flood, the effects of which will likely propagate through the 
housing market dynamics. We speculate that there is an increased risk of highly volatile market responses driven by fear and irrational behaviour in areas where climate change causes an increase in extreme flood events. In the extreme case, some areas risk large-scale outmigration (Black et al. 20I I), which causes a significant drop in property values in high risk zones (Pryce et al. 20I I). Yet, the impacts will vary per hurricane region around the globe, and it will even vary within our study area in the U.S. The impacts of climate change will vary across regions and the ways in which people deal with flood risk may differ as well.

Our results have important policy implications. Namely, while evading flood zones is an effective way to reduce a households' vulnerability to flooding, we stress that this strategy should be adopted in a smooth transformation to prevent migration and market shocks that are driven by extreme events. Instead of subsidizing repetitive damage properties over and over again, policies could focus on getting people to move out of flood zones well before the next major flooding event. These transformations are not always easy to adopt, and will have a particularly high impact in cities that are entirely below base flood elevation. Yet, in many cases this transformation should not be devastating, since small localised differences in elevation make a big difference in flood risk. Flood zones - and river floodplains in particular - are still very local in many cities.

People's choices where to live and how to protect themselves is a complex issue that depends on many factors. We focused on the cognitive drivers of these individual choices. How these choices affect the structure and outlook of coastal cities affected by climate change is subject for future studies. A survey that measures the status quo alone is insufficient to quantify the potential of reaching a 'tipping point'. Further studies are best focused on quantifying the cumulative impacts of the behavioural responses that we found. This study can be used as input to simulate and explore market responses under various scenarios of flood risk in our future climate and in combination with climate change impacts projections in coastal areas. 


\title{
CHAPTER 6 - Repetitive Floods Intensify
}

\section{Outmigration and Climate Gentrification in Coastal}

\section{Cities}

This chapter has been submitted as a paper to the journal 'Nature Climate Change' on $11 / 12 / 2018$

\begin{abstract}
The 2018 floods in the USA, Europe, and Asia reminded societies across the world of the need to revisit their climate adaptation strategies. Rapid urbanization coinciding with a growing frequency and intensity of floods requires transformative actions in cities worldwide. While abandoning flood prone areas is sometimes discussed as a public climate adaptation option, little attention is paid to studying cumulative impacts of outmigration as an individual choice. To explore the aggregated consequences of households' outmigration decisions in response to increasing flood hazards, we employ a computational agent-based model grounded in empirical heuristics of buyers' and sellers' behaviour in a flood-prone housing market. Our results suggest that pure market-driven processes can cause shifts in demographics in climate-sensitive hotspots placing low-income households further at risk. They get trapped in hazard zones, even when individual risk perceptions and behavioural preferences are independent of income, suggesting increasing climate gentrification as an outcome of market sorting.
\end{abstract}





\section{I Urbanizing coasts and climate gentrification}

Climate change is not a matter of the far distant future. High-impact storms are already increasing in frequency, with the 2017 hurricanes Harvey, Irma and Maria ranking among the 5 costliest hurricanes in U.S. history (NOAA 20I8). The impact of climate change on flood damage is expected to be even worse in the future when sea levels rise, and severe storms become more common (IPCC 20I4). Rapid population growth and urbanisation in coastal and wetland areas, driven by economic, cultural and environmental amenities that the coast and waterways offer (Nicholls 2004), lead to the increase of assets and the number of people exposed to intensifying flood hazards (Jongman et al. 2012). Adaptation to climate change that aligns both public and private actions requires an understanding of how people behave in response to increasing flood risks, how they are incentivised to adapt and what implications this has for the resilience of various groups of society. This is supported by theory and rich empirical literature on risk perception and its dynamics in response to floods (Whitmarsh 2008; Knuth et al. 2014), and on people's willingness to take climate adaptation measures such as insuring against flood risk or flood proofing their homes (Grothmann and Reusswig 2006; Terpstra 20 I I; Koerth et al. 2013).

Despite a strong empirical focus on households' adaptation measures, individuallydriven outmigration as an adaptation option is still underexplored (De Sherbinin et al. 20I I; Adger et al. 2015). Outmigration may become increasingly attractive in the long run when risks become too high and incremental adaptation measures too expensive (Kates et al. 2012). Transformational changes - such as to move away from hazard zones (McNamara and Keeler 2013) - could become a viable option. Households may voluntarily choose to do so at the point where risk is unacceptably high, and people switch to abandoning hazard areas. This puts high-income households in a favourable position over low-income households, who may find themselves trapped due to the lack of resources to move (Black et al. 2013). Moreover, floods can lead to climate gentrification (Keenan et al. 2018) as highincome households push up demand and prices for safe locations, further forcing socio-demographic shifts in urban areas. While flooding has economic consequences for all affected, the impacts are more detrimental for those that are economically vulnerable. The consequences of floods are therefore also characterized by environmental injustice (Walker and Burningham 20I I).

Yet, an open question remains: what is the risk threshold for people to decide to switch to outmigration? What would be cumulative socio-demographic impacts of these behavioural traits if we wait for floods to happen? A quantitative study that 
brings these aspects together is missing. To address this gap, we study how people's risk perceptions change dynamically with the occurrence of major floods, exploring when people switch to outmigration as an adaptation option. In an empirical agentbased simulation we show how individual choices, institutionalised in property markets, involuntarily lead to demographic shifts in response to natural hazards. We show that this process can gradually sort out high and low income households, fuelling climate gentrification and placing vulnerable households further at risk.

\subsection{Floods risks, housing markets and climate change: modelling behavioural responses in an artificial society}

Comprehensive surveys, hedonic analysis, and flood modelling deliver a variety of empirical evidence for the relationships between climate-driven floods and adaptation choices, property values and socio-demographics in hazard zones. Agentbased modelling $(A B M)$ is increasingly becoming the mainstream method to merge this variety of data on behavioural traits, with adaptive learning, dynamics of institutions and spatial or environmental changes essential to study socio-economic impacts of climate change (Stern 2016).

To explore the impacts of potential bottom-up outmigration from flood zones on the socio-demographic structure of cities in face of repetitive floods, we employ a spatial $A B M$ of a housing market where buyers and sellers with heterogeneous risk perceptions and incomes interact (de Koning et al. 2017; Filatova 2015). ${ }^{25}$ Buyers choose a dwelling within their budget based on its price and characteristics. Their attitude towards flood risk may inhibit them from buying property in a flood zone. A real estate agent informs sellers on an efficient ask price and updates their appraisal based on recent supply, demand and transactions. Households who reside within a flood zone may choose to put their house on sale and look for a home in a safer location, which according to our survey data is more likely to happen when they have experienced a flood. We simulate how people update their risk perception and their preferences for living in a flood zone after the occurrence of a major flood, which is grounded in theory and empirical observations of household-level preferences and behaviour in response to floods (de Koning et al. 2018). Individual changes in behaviour affect the supply, demand, and value of properties in hazard versus safe areas. Driven by adaptive households' preferences, the effects of floods propagate

${ }^{25}$ A more detailed methods description can be found in Appendix 6.a 
through market interactions, affecting the socio-demographic structure of climatesensitive urban areas.

Our ABM uses a number of unique datasets for calibration and validation: (I) two GIS datasets of actual properties (Bin et al. 2008a, Bin and Landry 20I3), along with information on structural characteristics and flood zone location based on federal flood maps (FEMA 20I8), (2) time series of housing sales before and after a flood (Bin and Landry 2013), (3) $2 \times 2$ hour in-depth interviews with real estate agents to specify the main architecture of the market (how ask and bid prices are formed, how agents negotiate prices, how they adjust prices, how learning on price expectations is happening) and $19 \times$ half-hour to one hour interviews with real estate agents on how flood zones affect the decisions of buyers and sellers in the market, and (4) surveys among 519 buyers and 521 sellers, of which some were affected by the floods caused by hurricane Harvey in 2017 (de Koning et al. 2018). To our knowledge it is the first time such an extensive model of the housing market is tested in the context of climate change in coupled human-and-natural systems.

To account for increasing frequency and the extent of flooding, we apply the model to two cases in North Carolina, USA: Beaufort and Greenville. Both cities are in an area where hurricanes caused major flood damage to properties. They differ in the nature of the flooding (coastal storm surge vs. inland river flooding) as well as in the extent of the flood zone - Beaufort has a larger share of hazard-prone properties, and hence the impact of flooding is more widespread. We simulate 15 years of property transactions in the period 2015-2030 with two repetitive floods in 2020 and 2024. We assess the net impact of recurring floods by comparing it with a benchmark scenario with no floods and a scenario with a single flood in 2020.

\subsection{A bilateral model of the housing market}

The RHEA (Risks and Hedonics in Empirical Agent-based land market) model is an agent-based model of a housing marked with a proportion of properties that are at risk of flooding. The model contains household agents who own residential property and can decide to put their property on the market, buyers who search for residential properties within their budget and preferences and enter negotiation with sellers (Fig. 6.I). A central real estate agent informs the sellers on the price they should ask for their property. The real estate agent adaptively updates the price expectation during the simulations on the bases of recorded sales as well as failed sale attempts. 


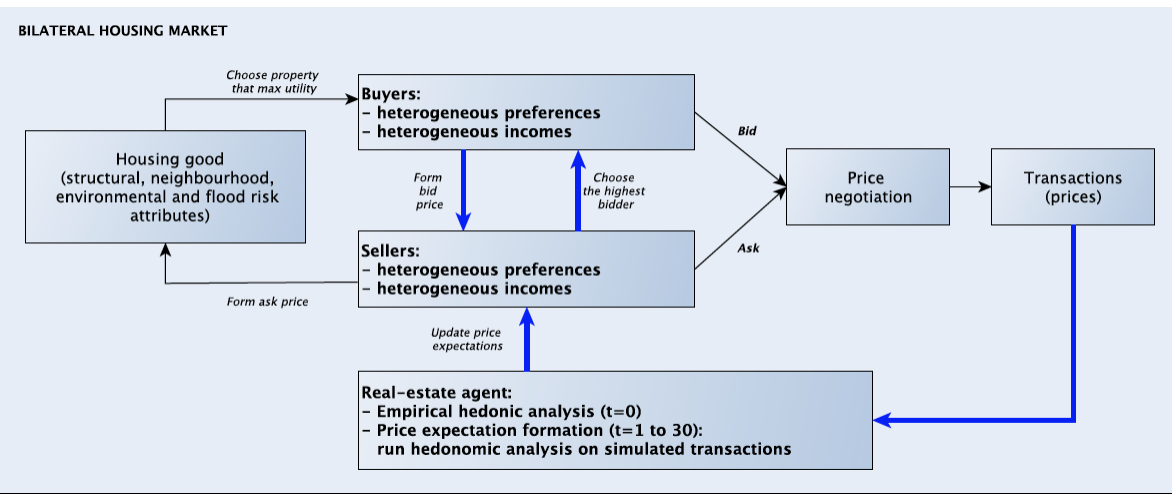

Figure 6.I. Schematic representation of interactions between buyers and sellers, and the real estate agent that updates the price expectation based on property transactions in the bilateral housing market model. Source: Filatova 2015

The trade procedure is as follows. Each time step represents a half year of property transactions. At the beginning of each time step 10\% of the homeowners randomly decide to put their property on the market. The realtor informs them on the price they should ask for their property, based on hedonic analysis of sales in the previous time step and a correction for demand for similar properties in the neighbourhood. Each time step a number of new buyers enter the market, approximately equivalent to the number of new properties on sale to avoid creating an excess of demand or supply artificially. Buyers randomly select 5 properties within their budget and preferences constraints. They compare them with a multi-attribute utility function to select the property they want to bid on. Buyers that were already on the market but have not found a property will follow the same procedure until 4 unsuccessful trade attempts ( 2 years), after which they will leave the market. Once buyers have selected a property and placed a bid, sellers will evaluate if they have received any bids and who has offered the highest. They are prepared to accept a lower bid as long as it is above a minimum price they consider acceptable. All trade attempts (including unsuccessful ones) are registered and used for the adaptive price expectation algorithm of the realtor agent in the next time step. After a successful transaction $47.5 \%$ of sellers stay in the market and become buyers in the next time step as our survey data indicated.

Flood risk perception, experience with flooding and damage from flooding are the main variables that affect households' decisions; they determine if buyers avoid buying properties in the hazard zone, and sellers may decide at some point that the risk of flooding becomes too high and they do not want to live in the flood zone anymore. Risk perception, feelings of fear toward flooding and experience with flooding are 
heterogeneous among agents, which are elicited from empirical survey data. Furthermore, buyers have heterogeneous preferences for property characteristics (age, surface area of living space, size of the lot, number of bedrooms and neighbourhood quality), and they have heterogeneous budgets.

The user can choose between two case studies of actual property markets in North Carolina, based on GIS data of actual properties and their characteristics, Greenville $(\mathrm{N}=9793)$ and Beaufort $(\mathrm{N}=348 \mathrm{I})$. The two cities differ in the fraction of properties that are located in the hazard zones: Greenville has $6.4 \%$ of the properties in the 100 -year flood zone, and Beaufort has $29.9 \%$ of the properties in the 100 -year flood zone and another $21.5 \%$ of the properties in the 500 -year flood zone.

\subsubsection{Updating price expectations}

Following Filatova 2015, the adaptive price expectation algorithm is based on the hedonic analysis of simulated transactions occurred in the past time steps. Given the statistical tests on model prediction accuracy for small samples, we reduce the number of explanatory variables common in traditional hedonic analysis to the few essential ones: square footage of living space, age of the house, lot size, number of bedrooms and a dummy variable ( 0 or $\mathrm{I}$ ) describing if the property was located in a (100/500-year) flood zone. The variables related to proximity to the city centre, parks, schools or highways are grouped into the single 'neighbourhood quality' represented by the residuals, which we spatially interpolate using kriging (Dubin 1998). These updates significantly improved the accuracy of the price predictions, especially when the sample size was rather small (de Koning et al. 2016).

Furthermore, price expectations account for demand for a segment of a market in the previous time step. For each property, the realtor checks the demand for a group of properties with the same characteristics. The observed demand for these properties relative to the expected demand (at time $t-l$ ) determines if the price should be lowered or raised (at time $t$ ). The maximum amount by which the price is changed is captured by a variable called 'alpha', which can be adjusted by the user. Alpha is set at $10 \%$ by default. This variable plays a large role in the simulations when flood risk perceptions and experience enters the decision making of buyers. When a large number of buyers avoid properties in the flood zone, the price of properties in the flood zone must be lowered to attract more buyers.

\subsubsection{Households' budgets and incomes}

The budget that buyers are ready to spend on housing is based on how much they earn. The agents (homeowners, buyers and sellers) have various levels of income based on U.S. national income data (Statista, 2017). When households enter the 
market as a buyer they allocate a certain budget to housing, which is a function of their income (Eq. 6.I). Each buyer spends a random percentage of its budget on housing, but low income buyers spend relatively more of their income on housing than high income households. The percentages of income that households allocate to housing is validated with survey data and U.S. national statistics (Statista, 2017).

$e^{4.96+0.63 * \ln (\text { income })}$

Furthermore, the housing budget does not exceed $30 \%$ of a households' income, which is a financial rule-of-thumb for homeowners and is often the mortgage limit supplied by mortgage lenders. In the model, the housing budget includes mortgage and flood insurance. The percentage of the housing transaction that is financed by mortgage, if anything, is also a random percentage drawn from a distribution based on survey data and U.S. national statistics (Statista, 2017). Some buyers are cash buyers and do not have a mortgage. Homeowners that do have a mortgage will pay off their mortgage linearly in 30 years. This is another important aspect of the model, because it determines whether homeowners are capable of selling their house. If the expected price of their property on the market has dropped below their mortgage debt, for example as a result of a major flooding event, they will abolish their trade attempt. These traders are identified as 'stuck' in their homes.

\subsubsection{Individual decisions under risk: empirical heuristics for households agents}

In 2017-2018 we held interviews with real estate agents and surveys among buyers and sellers as input to set up the model architecture of households' responses to flood risk. We started with 19 of 30-60 minute semi-structured interviews with real estate agents to start exploring which decisions are relevant in housing markets where a proportion of properties on sale are located in a flood zone. These interviews helped us in setting up two conceptual frameworks - one of buyers and one of sellers - of individual behavioural heuristics when facing flood risk, in particular how it affects households' decisions in the housing market (e.g. whether or not to evade or abandon the flood zones). We used the risk perception variables of protection motivation theory as the bases for explaining the behavioural responses in our conceptual framework.

Next, we ran surveys separately among buyers and sellers $\left(N_{B}=519, N_{S}=521\right)$. The surveys aimed to I) verify the conceptual model and adjust it based on statistical analysis, and 2) parameterize some of the input variables of the model, such as the variables that drive flood zone avoidance behaviour. Among our survey respondents $25 \%$ have experienced a flooding, including that from hurricane Harvey; $7 \%$ of the 
respondents had experienced damage from flooding. The survey measures how these experiences affect people's perceived likelihood of damage, their feelings of fear towards flooding, and their behavioural responses such as buyers avoiding properties that are located in the flood zone and homeowners that decide to sell their property because they do not want to deal with flooding anymore. More details of the survey questions, the conceptual frameworks and data analysis can be found in (de Koning et al. 20l8).

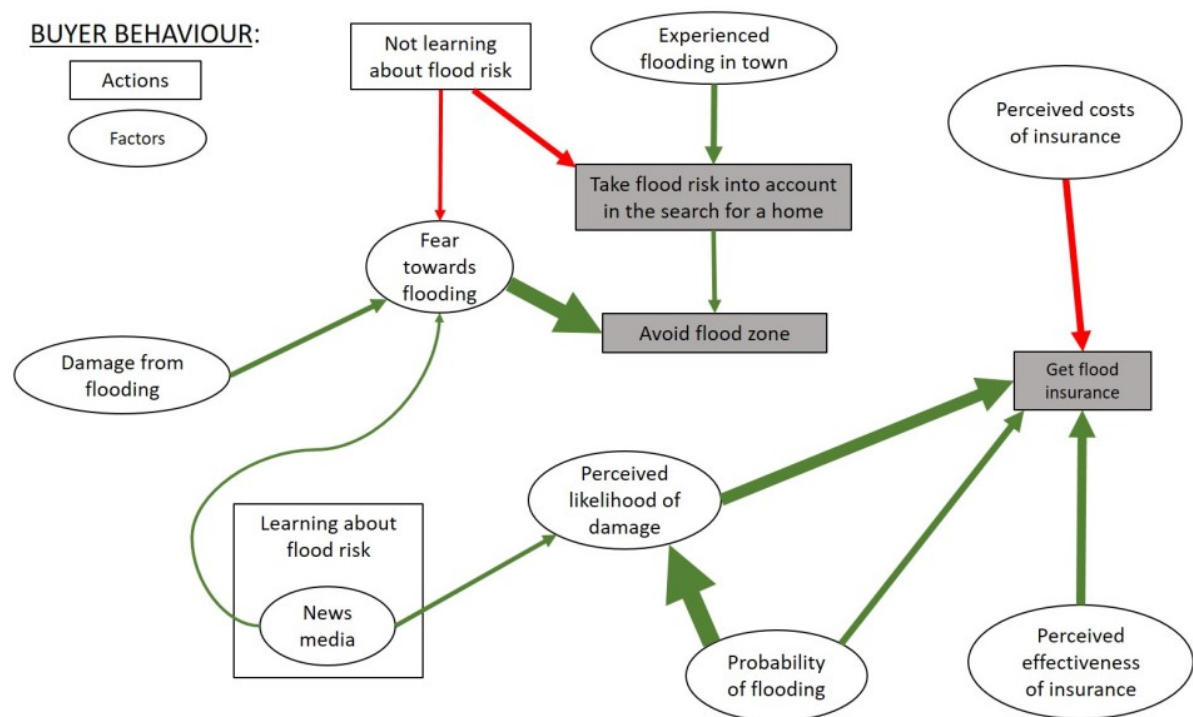

Figure 6.2. Schematic representation of buyer behaviour in response to flood risk. Red indicates negative effects and green the positive. The strength of the impact is given by the thickness of the lines. The main responses of buyer agents in the model are highlighted in grey. 


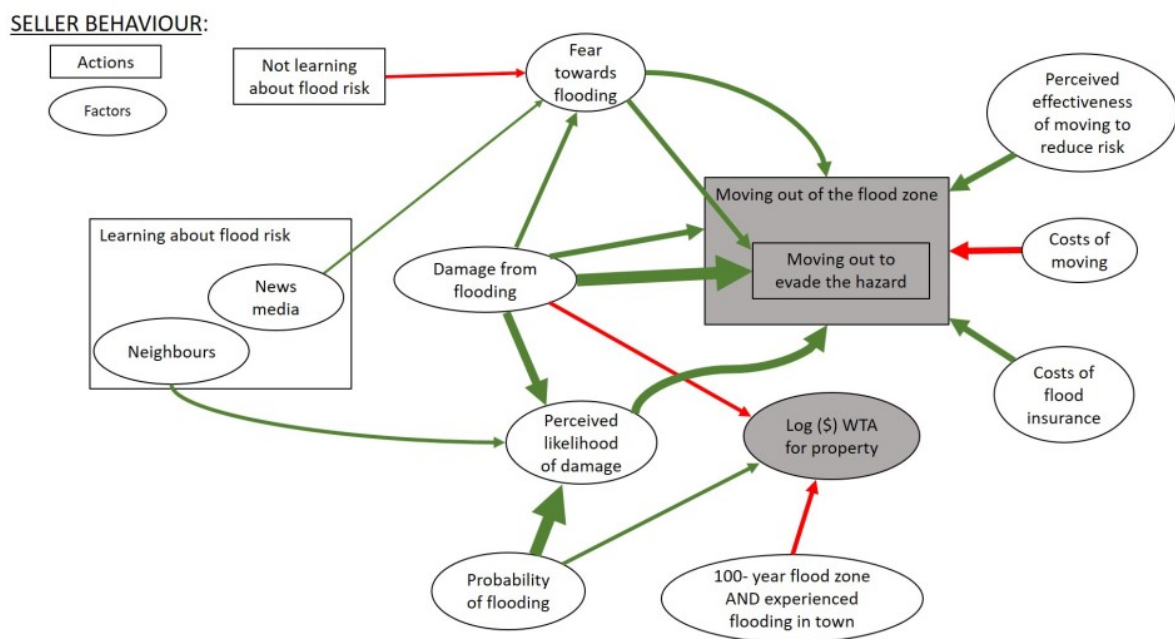

Figure 6.3. Schematic representation of seller behaviour in response to flood risk. Red indicates negative effects and green the positive. The strength of the impact is given by the thickness of the lines. The main responses of seller agents in the model are highlighted in grey. The box 'moving out of the flood zone' indicates household agents that sell their property for all sorts of reasons, and choose to live outside the flood zone after the move. The box 'moving out to evade the hazard' is a subset of the former group highlighting agents sell their house explicitly to escape the hazard of flooding.

We ran Bayesian statistics and regression analysis on survey results, upon which we built the module of flood risk and behavioural choices of buyers (Fig. 6.2) and sellers (Fig. 6.3). We used the output of the analysis directly in the model to set the Bayesian learning rules in which agents update their risk perception variables and their behavioural responses correspondingly. The risk perception variables and behavioural responses depend on the individual experience with flooding in town, on the damage to own property and on the damage to neighbouring properties. In the risk context people exhibit bounded rationality. In particular, homeowners in the flood zone weight their perceived probability and perceived damage while making decisions under risk (i.e. behave according to protection motivation theory) before they experience any flooding. As soon as they have personal experience going through this shock, more psychological reasoning kicks in: for example with fear dominating the decisions to move away from the flood zone (Fig. 6.3). Every half year homeowners' update their perceived likelihood of damage and likelihood to abandon their risk-prone property. Also, the fear score of all households and buyer's tendency to avoid the flood zone get updated according to their information sources (Fig. 6.2 and Fig. 6.3). 


\subsubsection{Setup of the computational/simulation Experiments}

We design the experiments with our agent-based model in R-studio. We simulate a I5-year period of sales (i.e. 30 semi-annual time steps) in the two different coastal markets (Greenville and Beaufort, NC) between the years 2015 and 2030. Since more frequent floods are projected to be a norm in a climate-changed world, we model repetitive floods: a major flooding event in 2020 and another in 2024. The major flooding events are characterised by a return period of once in 500 years in our current climate, although it is expected to happen more frequently in a changing climate. The GIS dataset of parcels contains information on whether the properties are in a 100/500-year recurring flood zone, and it is assumed that these indications are correct for all properties. Hence, we assume that all households living in the 100 and 500 year recurring flood zones experience damage to their homes by the floods. All households in town (homeowners and buyers) update their experience with flooding - a binary true/false variable - after the floods. Housing prices reduce in anticipation of a drop in demand for properties in the flood zones. To capture this in the model, we assume that following the flood affected sellers reduce their price by $13.8 \%$, and sellers in the 100 year flood zone reduce their price by an additional $15.7 \%$ (empirically observed in the survey among sellers).

We run 663 Monte Carlo model runs for both datasets - Greenville and Beaufort. The settings of alpha and the percentage price reduction for flood zone properties in the years of the flood vary per model run. The Monte Carlo runs also vary in the random seeds that produce stochastic effects in the virtual market. The (quasi)random values that are produced in R-studio can be fixed with the 'set.seed' function, which calls a list with quasi-random values that can be used to generate random processes in the simulations. This list include: deciding which 5 properties are selected by buyers, setting households' preferences, incomes and risk perception variables, or selecting which homeowners want to sell their property. One Monte Carlo run consists of three experiments with constant random seed: a control without flooding, an experiment with only one flood in 2020, and an experiment with both floods. With this approach we can keep all other processes between the experiments constant that allows us to re-trace the effect of the floods in isolation of stochastic effects. The control experiment starts to deviate from the flood experiments after the first flood, and the experiments with two floods deviates from the single flood experiment after the second flood.

We monitor how the value of properties change over time when they are sold repetitively on the market. The value change (measured as a percentage of its initial value at $\mathrm{T}=0$ ) is averaged for all properties in the 100-year and 500-year flood zone, 
which we plot as a comparison among the experiments. Furthermore, we monitor the percentage of seller agents in the 100-year and 500-year that failed to sell their property after 2 years (leaving the market with their property unsold) and the percentage of sellers that are forced to abolish their sale because the predicted market price of their property is valued below their mortgage debt (identified as 'people stuck in their homes'). Again, these statistics are plotted as a comparison among the three experiments - a control, a single flood and two floods. Lastly, we monitor the number of people below the 2016 US poverty threshold in the 100 -year and 500-year flood zone ( $\$ 24.563$ for four-person households). These are plotted as a percentage difference between the flood experiments and control experiment.

\subsubsection{Sensitivity analysis}

Finally, we run a sensitivity analysis on three variables that we expect to have a large impact on the results. Namely, alpha that controls the price correction according to demand for a sub-market, the price reduction of properties in the 100 year reoccurring flood zone in the year of the flood, and the price reduction of properties that were flooded. Alpha is set at $0,0.05,0.08,0.10,0.12,0.15,0.20$ and 0.25 . The other two variables are sampled at each iteration from a normal distribution, for which we use the Bayesian posterior probability distribution based on the survey data.

The primary goal of this sensitivity analysis is to do a robustness check. We want to assess how robust the conclusions of our study are when we change the assumptions on these three input variables - from which we think they have a large impact on the results.

\subsection{Transition from affluent neighbourhoods to poverty}

\section{traps}

Affluent locations in a coastal town may become unattractive for living as floods become repetitive and signal the extent of risk when affecting a large share of local properties. Housing markets drift into a new regime when damages lead to a drop in the aggregate demand, when market recovery does not occur smoothly, and when some people rush to relocate into safe zones while others remain trapped in the hazard zones.

\subsection{Damage and drop in demand}

Under a variety of behavioural heuristics elicited from the survey, our spatial agentbased coastal housing market model indicates that a major flooding initially stagnates 
the market. Properties suffer damages and demand for properties in the flood zone declines rapidly as household agents avoid risk-prone properties. It causes a significant drop in property values in the hazard zones (Fig. 6.4).

\section{Average value change of a property}

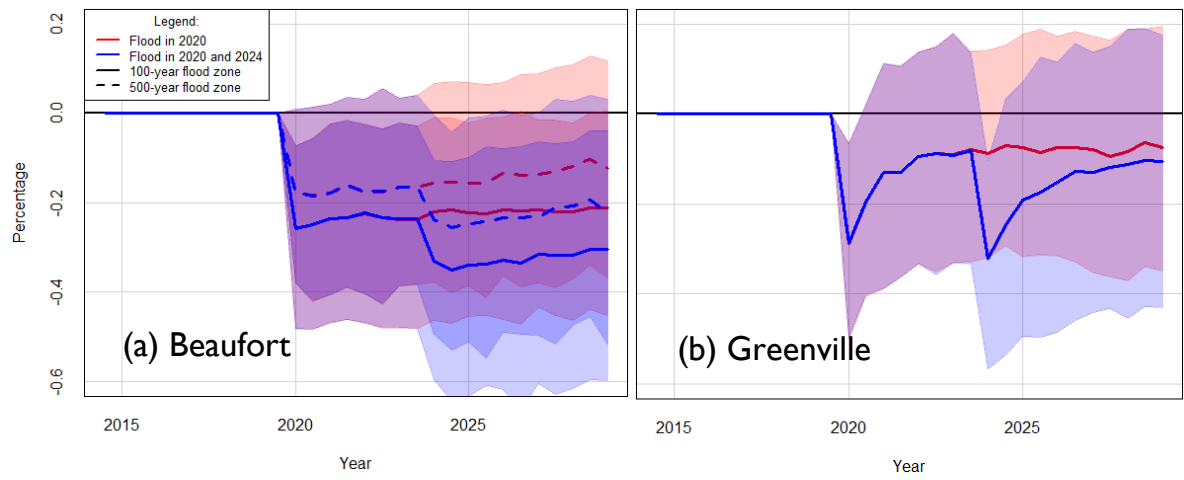

Figure 6.4. Change in property values in the 100 -year and 500 -year flood zones as a result of flooding. Recovery time differs between the cases because Greenville has a lower proportion of properties in the flood zone. Lines represent the average impact of the first flood (red) and of both floods combined (blue) across 663 Monte Carlo model runs. The bands represent $80 \%$ of the runs.

In both towns the peak price drop occurs immediately after the flood, after which values slowly start to recover. The price recovery is a result of newcomers entering the market from outside who have not yet experienced local floods. These people are generally less risk-aware than those who have experience with flooding and damage to property (de Koning et al. 2018) and see an investment opportunity in the temporarily low-valued properties in the hazard zones. As a result of market sorting, this group of risk-unaware buyers generally have a lower income than households who lived there before the flood. Due to path dependence over time, the effect is amplified by the fact that demand for safe properties increases followed by prices, forcing low-income households into hazard zones. Consequently, the first flooding results in a price drop of $26 \%$ (29\% for Greenville) on average in the 100-year flood zone and $20 \%$ in the 500 -year flood zone for Beaufort (Fig. 6.4.a and 6.4.b). The second flood leads to a $35 \%$ drop (32\% for Greenville) in the 100-year flood zone and $25 \%$ in the 500 -year flood zone.

\subsubsection{Market recovery}

Recovery time of property values strongly depends on the number of properties in the flood zone and the number of households affected by the flood. Markets with 
only few properties in hazard zones (Greenville, Fig. 6.4.b) quickly recover, as the city population forgets about few local flood occurrences in the large pool of unaffected properties. Hence, there is sufficient demand from risk-unaware households moving into flood zones, and it does not create a lasting market effect. Contrastingly, markets with a large share of flood-prone properties (Beaufort, Fig. 6.4.a) witness a troublesome shift in the market trend. When many people have experienced flooding or property damage the price drop is significant and lasting. Moreover, there is a surplus of properties for sale in the flood zone compared to the relatively few risk-seeking households that buy them, resulting in a large share of unsuccessful sale attempts in Beaufort after the flood (Fig. 6.5.a).

\subsubsection{Outmigration from flood-prone areas}

In the model, empirical behavioural traits prescribe some affected households to relocate from hazard areas after a flood. It results in a significant outmigration of people away from the flood zone, particularly when the number of affected households is relatively small. The fraction of households moving out is a lot smaller when there are more properties affected, limited by market demand for flood-prone properties. Namely, while a great number of household agents desire to move out, the relocation is limited by the number of people that are willing to buy these properties. Initially the sales increase slightly due to risk-unaware buyers that are attracted by the low prices, but in the long run people risk getting locked in the hazard zones because few people want to buy their houses (Fig. 6.5.a and Fig. 6.5.b). This is particularly the case in Beaufort that has a large share of affected households and relatively few risk-unaware buyers (Fig. 6.5.a).

Moreover, when prices drop sharply following a flood it impedes some household agents from selling at a price lower than their mortgage ${ }^{26}$ (Fig. 6.5.c and Fig. 6.5.d). Hence, households with a low down-payment become locked into living in hazard areas. Households that invested more personal capital in the property have better opportunities to migrate out of the hazard zones, since they can afford accepting a lower price for their house.

\footnotetext{
26 This immediate effect of people getting stuck does fade away relatively quickly when prices recover and people have paid off more of their debts. The pattern is independent of the study area, which indicates that paying off the mortgage is more important as prices do not recover very well in Beaufort after the flood.
} 


\section{People failed to sell}
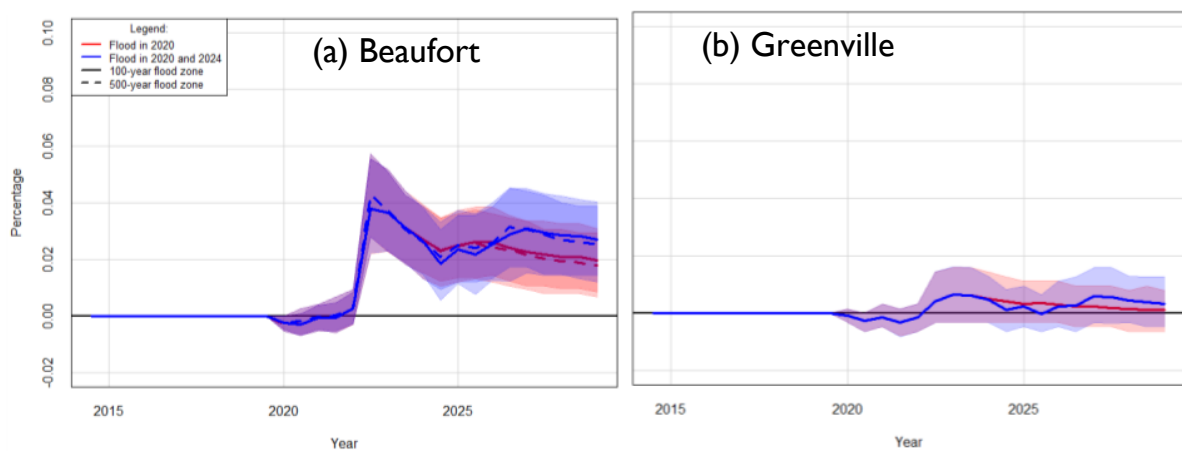

People stuck in their homes
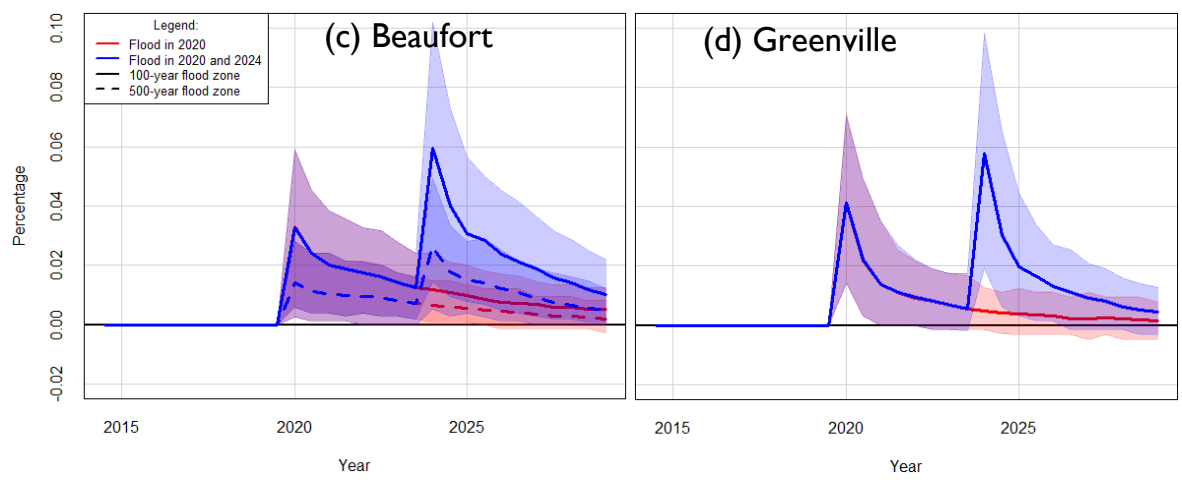

Figure 6.5. Households that want to move out of the flood zone after the flood but cannot. Either they withdraw their property from the market after a number of unsuccessful attempts due to low demand (Fig 6.5.a and 6.5.b), or because their mortgage debt is higher than the market value of the property (Fig 6.5.c and 6.5.d). Lines represent the average impact of the first flood (red) and of both floods combined (blue) across 663 Monte Carlo model runs. The bands represent $80 \%$ of the runs.

\subsubsection{Climate gentrification}

The two above-mentioned processes - a drop in demand and prices after the flood, and new low-income risk seekers moving in - together cause a gradual increase in poverty in the years following major flooding events. Flood damages and the drop in property values results in a gradual decrease in incomes of households residing in the flood zone, with the lower income cohorts affected stronger. The median income of households in the 100 -year flood zone decreases by $2-3 \%$ after the first flood and $4-6 \%$ in ten years with two major floods, while in that same period the lowest income 
quintile decreases by $4 \%$ up to $7-9 \%$ respectively. As such, the poor people get poorer, increasing social vulnerability in flood-prone areas. Consequently, the percentage of households earning beneath the poverty threshold increases steadily in both modelled towns in the years following the flood (Fig. 6.6).

Change in poverty (households earning below \$ 24.563)

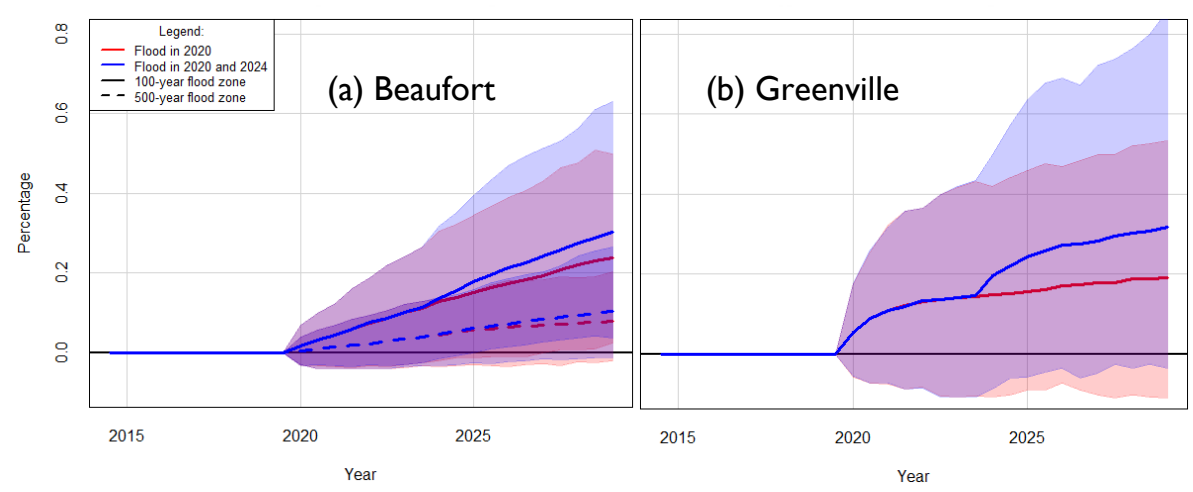

Figure 6.6. Change in poverty in the 100 -year and 500 -year reoccurring flood zones as a result of flooding. We use the 2016 US poverty threshold for 4-person households as a reference (US Census Bureau 20I7). Lines represent the average impact of the first flood (red) and of both floods combined (blue) across 663 Monte Carlo model runs. The bands represent $80 \%$ of the runs.

Although we saw that prices and selling conditions recover somewhat in a span of 510 years after the flood, in particular in Greenville, the increase in poverty seems to be more permanent in both study areas. Even when prices have a tendency to return to their old level, poverty still increases by over $30 \%$ compared to the control scenario ten years after the first flood. The processes gradually change the centre of gravitation on a market pushing high-income households towards safe zones, while attracting increasing numbers of highly vulnerable households to riskier locations. This goes hand-in-hand with climate gentrification based on speculative investments in low-hazard properties (Keenan et al. 2018), which further reinforces the trends of high-hazard neighbourhoods falling into decline. 


\subsection{Social simulations as testbeds for inclusive cross-scale climate adaptation policies}

This paper contributes to the climate adaptation literature by studying the consequences of household-level outmigration decisions on socio-demographics and urban resilience, mediated through housing market responses. Our coastal housing market $A B M$ shows that household behaviour in response to floods triggers market sorting, which enhances the risk of climate gentrification. Low-income households get trapped in hazard zones, even when households' risk perceptions and behavioural preferences are independent of income. The behavioural rules in our simulations are validated with empirical findings of outmigration triggered by floods that are already happening in our current climate, including the floods caused by Harvey. We use the spatial $A B M$ to study urban sorting, which could be expected as floods intensify in frequency and volatility with climate change. Hence, we can already find some empirical parallels of our model's scenarios such as the outmigration that happened after hurricane Katrina ${ }^{27}$ (Zainetti and Colten 2012). The results of this paper highlight that a bottom-up 'Laissez-Faire' approach to climate adaptation could locally result in increased social vulnerability to flood risk, the extent of which will likely expand rapidly given the climate and population trends along the coast Jongman et al. 2012). We stress that a timely and coordinated approach of well-structured institutional action is necessary in order to increase urban resilience against climatechange-driven floods. An artificial society, such as in the presented ABM, can be instrumental in exploring adaptation pathways where costs and benefits are shared by public and private actors.

While our model shows important market-driven effects of floods on urban resilience, the interaction with other institutions and sociodemographic processes might amplify or attenuate the effects. Our model can be extended by including other relevant drivers of socio-economic vulnerability to floods, and ways to channel smooth urban transformations in climate-sensitive areas. Future research may focus on: (I) connecting to labour markets - storms put businesses out of operation or cause major interruptions, and job (un)availability inhibits people's options to outmigrate, (2) integrating of sociodemographic push and pull effects - the impacts are amplified by urban blight when critical poverty thresholds are reached in the hazard

\footnotetext{
27 In the model we did not simulate a complete abandoning of properties. All properties in the model had to be re-occupied. We are uncertain how this effects the results, but the model allows possibilities to further explore this.
} 
area, and (3) modelling of institutional responses - insurance companies, (federal) risk-management agencies and policy interventions can play a major role in the recovery trajectory after a flood, and in damage prevention before the flood. Institutions in particular can be instrumental in improving social resilience against future flooding and assuring the benefits of cross-scale adaptation (Adger et al. 2005). The model is explicitly designed to explore bottom-up drivers of resilience against flooding. Given that it projects an emerging increase in social vulnerability, it is worthwhile to investigate what role institutions can have in alleviating the impacts of future floods. 
CHAPTER 7 - Discussion and Synthesis 

In this thesis I address the modelling of human behaviour in CHANS, with the purpose of exploring how the natural environment might change in the Anthropocene as a consequence of collective human action, and consequently how this changes societies. The impact of humans on the environment affects the wellbeing of our species, placing a huge responsibility on us to make the right decisions. The role for scientists lies in understanding our impact on the environment, and exploring how to prevent undesired changes. Therefore, the models that we make must be capable of exploring policy options that help to steer our behaviour in the right direction. We are capable of building complex models of the environment such as global climate models - emphasising the physical processes in natural systems. Yet, the complexity of human behaviour and its impact on the environment is still underrepresented in the models. In particular adaptive behaviour, social interactions and learning are not sufficiently taken into account in many models (Stern 2016; Verburg et al. 2016). Hence, in this thesis I addressed how humans are represented and modelled in complex models of CHANS. I illustrate this methodological issue with a case study of flood risk in climate-prone urban areas. The model in this case study illustrated how an increase in flood risk - driven by climate change - changes peoples' risk perceptions and behavioural responses, which aggregates into shifting patterns in socio-economic institutions such as housing markets. Changes in these institutions leads to shifts in urbanisation patterns, placing socio-economic vulnerable groups more at risk than others. In this final chapter I synthesise what we have learned methodologically from working on this ABM, and how this helps in addressing the research goal:

Improving the modelling of human behaviour in coupled human and natural systems, in agent-based models that are designed for exploring global environmental change scenarios and policy intervention strategies

I will first discuss step by step the answers to the research questions that are covered by the different chapters. After which I will synthesise how the chapters in my thesis contribute to improving the way we model human behaviour in CHANS. I will highlight a few ongoing discussion points: (I) should we make ABMs as simple as possible or increase the complexity, and (2) how to balance theory and data, and which theories and data to use when validating and parameterising human behaviour in ABMs of CHANS. Next, I suggest a list of concrete modelling steps to take when trying to improve the formalisation of human behaviour in models of CHANS. Finally, I will highlight some new lines of research that I suggest for future studies pursuing the goal of addressing with simulation models the grand scientific challenges of the Anthropocene. 
Research question I: How to capture the collective preferences of a variety of individuals in a dynamic environment? (Chapter 2)

In chapter 2 I was faced with the challenge of capturing preferences on an aggregate scale. Although ABM is focused on behaviour and preferences of individuals, part of the environment that agents perceive and where they interact consists of the agents and their preferences as a collective. The preferences in the case study were represented by buyers' willingness to pay and the sellers' willingness to accept for a house. Yet, properties are vastly different in terms of structural characteristics, location, risk of natural hazards and neighbourhood characteristics, and therefore these can be divided into a number of housing attributes. In an efficient market it is assumed that the market price for an attribute is where willingness to pay and willingness to accept meet, which is called the marginal implicit price of an attribute. By combining all the marginal implicit prices of all attributes, one can estimate how properties are valued by buyers and sellers in the market. This is the logic I followed in the model, which means that I took a utility maximisation approach in the assumptions of agents' preferences on an aggregate level. I assumed that the collective preferences of agents - despite that they differ per individual - can best be described by a simple linear rational choice model. The choice for utility maximisation theory is grounded in hedonic analysis, which is the practice of using housing values to monetise social costs and benefits of environmental variables (Rosen 1974).

I have found that it is difficult to capture aggregate preferences when sample sizes are small. It is common practice in the hedonic literature to capture a large number of housing characteristic variables. However, this practice does not suffice when one attempts to capture the dynamics of people's preferences. In particular when people's preferences are constantly changing, you should rather focus on limiting the number of variables that capture sufficiently how choices are valued by their attributes. The aggregated estimates of people's preferences are confounded by statistical biases in the independent variables and by behaviour that deviates from perfect rationality - or at least we fail to capture comprehensively all variables that matter in people's decisions (even my best model left more than $10 \%$ of the variance unexplained). A large part of the variance in the model predictions - in particular when sample sizes are small - is driven by heterogeneity in preferences of individuals and by multi-collinearity in the measured attributes. Multi-collinearity is present in many empirical datasets, since many attributes of choices are not statistically independent. For example: the number of bathrooms is linked with the size of the house, and luxurious houses are often associated with safer neighbourhoods and 
higher environmental quality. In these cases it is hard to separate one variable from another, and therefore it is important to consider whether the variables measure what you want to measure, and to consider if the model performs better when some variables are omitted.

My findings in chapter 2 show that models with fewer variables perform consistently better than models that try to capture as many variables as possible, especially those that are not statistically significant or where the regression coefficients have the wrong sign (i.e.: negative where a positive effect is expected). Too often models are over-complicated and over-fitted with variables without which the model's predictive value would be far better off. Furthermore, it is not uncommon in many social sciences disciplines to include a number of variables as covariates to control for their effect on the dependent variable, even when they lack a clear hypothesis as to how they have an effect. For example: in hedonic studies the distance to railroads is sometimes used to control for the effect that railroads may have on property values (Bin and Landry 2013). Yet, this does not specify what preferences may drive this effect. At a distance close to the railroad one can image that noise, structural vibration and perhaps even the hazard of a crash has a negative effect on property values, while on a larger spatial scale a high density of railroads could also be an indicator of good infrastructure and accessibility, which might positively affect property values. Furthermore, the effect can be so small that is easily overshadowed by more confounding spatial effects that just so happen to be statistically correlated with distances to railroads. A few things are important to question here: $(I)$ do the variables represent what we think they represent, (2) are the variables essential in capturing the main preferences of the population of people in the context, (3) are the variables represented by the correct mathematical function, (4) is it the most direct measurement of people's preferences or at least the best proxy for people's preferences, and (5) are the variables statistically independent?

When capturing the dynamics of people's preferences on an aggregate scale, it is important to be comprehensive but also to keep the model as simple as possible. The point is that you must always consider the common preferences that represent a general population: what are the main drivers of people's preferences in a certain context that can be generalised into a model? Large living space is generally preferred over small living space, newer is generally perceived as better, safer is better etc. You then have to try to reduce the number of variables and limit the complicatedness of the model as much as possible and simplify the functional forms. At the same time, you have to focus on theory, expert knowledge or common sense, and leave out variables of which you cannot intuitively predict how they are valued by people. The 
next step is to consider how people's preferences can be measured into attributes, e.g.: living space by the size of a house and garden, newness by the age of a house, safety by crime rates in a neighbourhood or by probability of a natural hazard. Some variables cannot be measured or quantified easily. For those variables the challenge lies in finding good proxies. In my case I captured the complexity of space by spatial interpolation of the residuals of the regression, represented by all remaining attributes excluding the structure of the house. These residuals proved to be a better proxy than a collection of spatial variables combined, such as distance to railroads, roads, city centre, rivers and parks. It is important to realise that this first part of my thesis/analysis is more about statistical parameterisation of general patterns than about capturing all the complexity aspects of CHANS such as heterogeneity, nonlinearity, adaptation and learning, which will comes later in the chapter when we focus more on the micro-level behaviour of the model in detail.

Research question 2: What are the aggregate impacts of various behavioural theories and assumptions regarding risky choices? (Chapter 3 and 4)

In chapters 3 and $4 \mathrm{I}$ dove more into disaggregate preferences on the micro level, which in chapters 3 and 4 was still very much theory-based. This is the first step in addressing the knowledge gap - should we use theory, data or both to inform behavioural rules in ABMs of CHANS, and which theories should we use? Some theories like utility maximisation do not need a lot of data to parameterise, and therefore this is a practical first theory to implement when modelling human behaviour in CHANS, which is typically done in most ABMs in the field of economics. In the case study I added prospect theory, which is a little more complex, but this theory also has well documented equations (Laciana et al. 2006) that can be directly implemented in the model.

What chapters 3 and 4 illustrate clearly is that the aggregate impacts of the model's micro-level behavioural assumptions are highly sensitive to the choice of a theory, and even to how a theory is formalised and implemented in the model. Furthermore, there are many biases in the dataset that interfere with the statistics that capture people's preferences on an aggregated scale. Similar to what I found in chapter 2, there is no perfect way of capturing the collective preferences because of many statistical biases in the dataset. This is problematic for two reasons. Firstly, the macro-scale patterns that we find in our agent-based simulations, as well as in empirical data, are masked by statistical biases such as multi-collinearity and noise, and hence we fail to accurately capture the collective preferences of people in 
empirical datasets. This makes it difficult to validate the behavioural assumptions and to parameterise any of the behavioural theories with aggregate data, because the macro-level statistics are inaccurate in the first place. Secondly, the coupling of micro-level and macro-level behaviour - i.e.: the feedback of environmental conditions on human behaviour - is modelled inefficiently because of systematic errors in capturing the environment.

In chapter $4 \mathrm{I}$ applied two simple behavioural theories (expected utility theory and risk negligence) in an experiment where I assessed the impact of mandatory insurances on property values in flood hazard areas. This chapter addresses whether an $A B M$ in which the behavioural assumptions of agents are based on theory alone is sufficient to assess the impact of policy interventions in CHANS. In other words: I tried to assess whether a mostly theory-based $A B M$ is useful in addressing the grand scientific challenges in the Anthropocene. I have used the model in my case study to illustrate the effect of a policy intervention aimed at internalising flood risk into property values, thereby countering the effect of market failure when people are not fully informed about risk. I found a consistent effect of the policy, exactly doing what it was intended to do. However, the net impact of the policy in the simulations was highly sensitive to the micro-level behavioural assumptions. How much risk people actually perceive, and what role it plays in their decisions influences drives the balance between costs and benefits of a policy intervention, which determines the decision whether a policy should be adopted or not. This uncertainty in the net effectiveness of policies, caused by an uncertainty of the model's theoretical validity, can be problematic when addressing the grand challenges in the Anthropocene with limited resources. In chapter 3 I have established a discrepancy between micro- and macrolevel measurements of people's preferences driven by all sorts of biases. Therefore, we cannot rely on policy explorations with a model where the micro-level foundations are parameterised on macro-level patterns. In this case we need to dig deeper into understanding human behaviour with the aid of detailed micro-level data.

Research question 3: How well do various behavioural theories fit with empirical micro level data of household behaviour in various contexts? (Chapter 5)

Chapter 5 covers two issues: it deals with the choice of a theory, supported by empirical data, that best fits with the context of the decisions that people make, and it elaborates further on the discussion whether to use theory, data or both to validate behavioural rules of agents in our models. I ran a survey among 519 buyers and 521 sellers in the residential property market in the U.S. to get a more detailed insight in 
people's preferences in the housing market, in particular how they perceive the risk of flooding in certain areas and how this influences their decisions of where to buy property and when to move away from flood hazard areas. Prior to the surveys I interviewed real estate agents (expert knowledge) to ensure the survey captures the essential questions that cover people's behavioural preferences in the flood-prone housing market. The advantage of the surveys was that I could collect a large number of variables that could drive behaviour in risky situations. Together they cover most of the theories of how people behave under risk and uncertainty, such as: feelings of fear, probability of a hazard, perceived control over the risk, risk perception and experience with natural hazards in social networks, and news media. Hence, in this setting I could use the surveys to empirically validate a number of theories to assess which one fits best in the context of the model.

I have found in chapter 5 that people behave differently in different contexts, and that the context decides what drives people's behaviour under risk and uncertainty. For example I have found that the preparedness to take precautionary action against flooding (getting flood insurance, elevating the property, flood proofing the house) is mostly driven by rationally weighing costs and benefits - i.e. how high is the probability that a flood happens and how cost-effective are the protective measures - whereas the choice whether or not to live in a flood hazard zone was mostly driven by feelings of fear towards flooding, which is more subjective and emotional. Thus, the decisions in former context were best explained by protection motivation theory, while the affect heuristics theory was more applicable in the latter context. In chapter 3.2 I state that there are various behavioural models that one can choose from when modelling human behaviour, and that each of them may result in different predictions on the behavioural outcome. Reviews about how to formalise behavioural rules in (agent-based) models (An 2012; Schlüter et al. 2017) do not provide assistance with the selection of a behavioural theory, seemingly because the model is indifferent which theories are used. However, the results of chapter 5 imply that behaviour is highly context-dependent, and that the descriptive accuracy of a theory changes with the context. Although behavioural theories are used interchangeably in various ABMs, I found that some theories simply fit the decision context better than others. Moreover, I found that people alter their perception of floods when they have experienced damage from a flood, and consequently the context of their decisions changes dynamically with flood events. People that experienced severe damage from a flood do not consider the choice of precautionary actions anymore, but rather choose to evade the flood hazard areas completely. Hence, for more accurate relusts it is good to consider being flexible in the use of a theory, and to consider that human behaviour and the theories describing it changes 
dynamically with the context and the environmental conditions. This is a particularly relevant issue to consider when exploring future scenarios in CHANS, given that these systems are prone to structural changes and regime shifts.

Moving to the discussion of whether to use theory, data or both, chapter 5 highlights the value of empirical data to validate behavioural theories that are used in an ABM. This is important to be aware of as a modeller. Even theory-based ABMs need to be parameterised to formalise decision rules in a model, and empirical data can be instrumental in doing so, whether aggregate or disaggregate. In particular when ABM is used for predictions in complex CHANS, the accuracy of the predictions strongly rely on how the input variables are parameterised. Some level of empirical data is therefore always needed to validate behavioural rules in ABMs. Yet, in chapters 3 and $4 \mathrm{I}$ have shown that aggregate data may not be adequate in this pursuit. Similarly, An and López-Car (2012) highlight that investigators need to be precautious in using top-down approaches when modelling human behaviour in CHANS. Which is why in chapter $5 \mathrm{I}$ use disaggregate empirical data as a more direct approach to infer behavioural rules. I have shown that the surveys can help modellers in selecting a behavioural theory, and in parameterising the variables of a theory when it is implemented in a model. The power of $A B M$ lies in simulating the emergence of macro-level patterns in a system through micro-level behaviour and interactions of many heterogeneous individuals (Gilbert and Terna 2000; Matthews et al. 2007; Farmer and Foley 2009; Railsback and Grimm 201 I). Hence, the formalisation of human behaviour in ABMs should do justice to this complexity at the individual level. One of these complexity aspects that can be captured only by disaggregate empirical data is heterogeneity. Focusing on aggregate data, general behavioural theories and average distributions have the draw-back that important heterogeneous details are lost (An 2012). Therefore, I argue that if you want to model human behaviour thoroughly in ABMs of CHANS, it seems that micro-level empirical data (e.g. survey data) are indispensable, in particular when empirical data is combined with wellestablished behavioural theories. I conclude from chapter 5 that there is a strong need for focusing on bottom-up behavioural choices that are informed by disaggregate empirical data, otherwise you risk losing the main strengths of $A B M$.

Finally, empirical data can sometimes substitute theories of human behaviour in ABM. In particular when none of the behavioural theories fully explains how people behave in all contexts. Some AMBs capture the dynamics of people's behaviour by altering the parameters of the theories they use dynamically in the simulations (e.g. Haer et al. 2017). For example: expected utility theory includes a parameter that specifies the extent to which people are risk-seeking or risk-prone. This parameter can be 
updated before and after a flood, to represent how people's risk perception and behaviour changes as a response to floods. Instead, I have used the surveys to calculate the Bayesian probabilities of a specific response, given people's experience with floods and a number of other parameters that characterise people's risk perceptions. The probabilities are used in the simulations to stochastically determine whether people adopt a certain response or not, which adds heterogeneity in the model. In that regard, the surveys combined with Bayesian statistics provide a nice short-cut to work around the mathematical 'tricks' of tweaking and fine-tuning parameters of theoretical models in the simulations. These cognitive short-cuts may actually represent behavioural rules well, a finding which has been established in heuristics theory in psychology (Slovic et al. 2005; Gigenzer and Gaissmaier 201 I). In some cases people rely on cognitive short-cuts to make quick decisions, rather than rationally weighing costs and benefits of various alternatives.

Research question 4: Can this $A B M$ be used to simulate the emergence of regime shifts from the bottom up, when changes in the natural system affect changes in human behaviour? (Chapter 6)

Throughout this thesis I have made continuous improvements to the ABM of the housing market. Chapters 2-5 highlight step-by-step which parts of the model were changed and how. These changes have led to the version of the model that I have used in chapter 6, where I explore the usefulness of the model in predicting how flood-prone urban areas will change as a consequence of climate change. I ran a hypothetical scenario of two major floods hitting a town shortly after each other, to assess how flood-prone cities may change demographically from the bottom up through autonomous households' responses to flooding. This is increasingly likely to happen as a consequence of climate change and urban development in flood hazard areas (Wong et al. 2014; Winsemius et al. 2016). Chapter 6 does not provide a definite answer to the question whether such model predictions are accurate or not. Instead, it is an exploration of the sort of macro-level outcome that may emerge through micro-level behavioural triggers in an ABM that is designed for the purposes of prediction. Although in chapter 6 I test the model, I do realise that I do not have the macro-level data to validate the predictions of trends in the future. Instead, one can qualitatively assess whether the predictions of the model present a plausible outcome, given our understanding of the dynamics in the system. Furthermore, the model structure and the agents' behavioural rules have been extensively validated in chapters 2-5 in order to gain more confidence in the model's prediction 
performance. The model in chapter 6 should thus yield more reliable predictions than earlier versions.

What I do test in chapter 6 is the model's potential to capture the emergence of regime shifts from the bottom up through changes in human behaviour in response to changing environmental conditions. A reason I use $A B M$ in this endeavour is because $A B M$ is focused on micro-level processes that drive system dynamics. Our focus on understanding how humans respond to a changing environment, and how correspondingly their behaviour and interactions enforce changes in the environment, allow us to detect micro-level signals before they have emerged in the system. Human-driven systemic changes and regime shifts in CHANS may not readily be observable at present, and hence simulations that predict regime shifts in the future are hard to validate. Yet, part of the behavioural drivers may already be visible on the micro level. In chapter 5, I assess empirically at the micro level how people responded to flood risk, and how their perceptions and behaviour changed when they had experienced a major flooding in their town or when they had suffered damage from flooding. Currently, this does not happen on a large scale, but it is expected to happen more widely in the future due to sea level rise, increasing number of severe storms and increasing urbanisation trends in flood risk areas. Therefore I use the empirical observations in chapter 5 and the model in chapter 6 to scale up the experiment in a hypothetical scenario of two major floods happening shortly after another. This way, I have been able to explore how the system (in this case climate-sensitive urban areas) might change in the future, while still being able to empirically validate the assumptions of the model on the micro level. It is important to assess whether the model outcome represents plausible behaviour at the agent level as well as at the system level. Keeping track of the cause-effectrelationships on different levels of the modelled system is the key in the interpretation of the model output and in assessing qualitatively the validity of the model predictions.

In chapter 6 we have seen that regime shifts are not unidimensional and do not necessarily manifest themselves in all facets of the system. Moreover, ABMs produce a highly complex multi-dimensional output, which offers the possibility to study the dynamics of multiple aspects of a system in detail. Hence, it is worthwhile to study a multitude of output variables in order to interpret how a system evolves over time, rather than tracking a single variable, e.g. the value of properties in flood hazard zones. I have shown that markets seemingly recover after a hazard event just by looking at price levels. Yet, by looking at how incomes are distributed between households among hazard areas and safe areas, one can see that some demographic 
shifts have occurred that would not have been observable by just looking at price levels before and after a major flood. These interpretations of the statistics would not be possible if it were not for the complex nature of $A B M$ as an approach to modelling CHANS. ABM offers a multidimensional approach to studying systems, which modellers and policy makers would find beneficial to make use of in order to benefit from the strengths of ABM. When exploring pathways of change in CHANS in the Anthropocene, it is important to account for the differences in patterns in the multiple facets of the system, for which ABM provides a more detailed overview than other modelling approaches.

\section{I Methodological contributions of this thesis to science}

Following from what we have learned by addressing the research questions, in this section, I would like to highlight a few methodological issues in ABM that this thesis sheds light on. I would like to address what could be learned from this research and how this contributes to doing better science with modelling, and by making some methodological advancements in ABM of CHANS in particular. First, I address the issue of finding the balance between simplicity and complexity (Sun et al. 2016; O'Sullivan et al. 2015). Secondly, I address the issue of how to validate human behaviour in ABM by using theories, data or both, and which theories to choose and what kind of data to collect. Thirdly, I propose a number of steps that are useful to consider when building or improving ABMs of CHANS.

\section{I.I Capturing complexity through simplicity}

Finding the right balance between simplicity or complexity is a much debated issue in ABM (Sun et al. 2016; O'Sullivan et al. 2015). The main argument for simplicity is presented by Occam's razor stating that the best solution to a problem is the simplest possible, and Einstein's razor that models should be as simple as possible but not simpler. This suggests that the optimal complexity of a model lies in minimizing the number of variables/factors while still being able to address the core issue. More complicated models are therefore not appreciated as better than the simplest ones. Yet, $A B M$ is especially designed to address systems in a more complicated way than other modelling approaches, which makes it more capable of capturing the complexity aspects of CHANS than other models. Therefore, highly simplified $A B M s$ may not have an added value over alternative modelling approaches. ABMs have been highly stylised and theoretical in the past, and now there is a general trend in making the models more complicated, supported by an increasing trend of using empirical data, big data and increasing computational power. These trends 
support the ability of $A B M$ to capture the complex behaviour of CHANS, in particular in modelling dynamics of systems that are out-of-equilibrium. But there is a limit to how complicated ABMs can be in order to be useful. This issue is discussed well by Sun et al. (2016), who present an arbitrary 'sweet spot' or 'goldilocks' zone' of complicatedness versus simplicity of $A B M s$ at the intermediate level of complicatedness. However, this is a rather abstract representation and does not provide a hands on guide as to what level of complicatedness is needed, and on which scales we need more detail versus abstractness. One contribution of my thesis to refining the model is that I show specific levels of the model (micro-macro) where the model needs to be simplified as much as possible and where it needs more complicated detail. On one hand I have found that simplifying the model as much as possible at the macro level produces more accurate predictions, by reducing the number of variables in the hedonic pricing function and by simplifying the representation of space. Contrastingly, at the micro level, I have improved the model by making it more complicated: adding heterogeneity to the agents, moving from theories based on economicaly-driven decision making to theories from psychology that capture more bounded rational behaviour, as well as adding more micro level empirical data to inform decision rules.

What I have shown in this thesis is that $A B M$ inherently requires a more complicated model, in order to address the complex systemic behaviour rules operating at the micro-level. The simplest theoretical assumptions of human behaviour, such as utility maximisation, did not perform well in explaining the patterns at the macro level. This is the reason that $\mathrm{I}$ used empirical data to get a more detailed representation of human behaviour in the model and to validate the use of more complicated behavioural theories. Yet, empirical data as an addition to the model's complexity at the micro level does not necessarily involve making the model more complicated. In fact, the data supported the selection of only a few core variables that were relevant in people's decision-making rules, such as their role as buyers or sellers, their level of fear and their experience with floods. Bayesian statistics can be used as a shortcut to define simple behavioural rules for agents based on these variables, similar to heuristic rules in human decision making (Gigerenzer and Gaissmaier 20I I). Indeed, I would suggest to use this Bayesian approach more in the future instead of tweaking the parameters of more complicated decision making theories.

Lastly, I want to highlight that making $A B M s$ simpler or more complicated is an iterative process. In chapters 2-6 I have improved the model step by step by expanding the model and adding detail, while in some parts of the model I have simplified it. You can use the stepwise approach by starting with a simple model and 
gradually making it more complicated until you reach a point where adding detail to the model does not add any value (Buchmann et al. 2016, Sun et al. 2016). And vice versa, you can start with a more complicated model which you gradually strip it down and reduce it to the core variables that are relevant in the system. I argue that both directions are necessary to explore at all times, but any incremental change to the model should help to enhance its predictive performance. There are no a-priori rules that define the 'sweet spot' in complexity versus simplicity in ABMs of CHANS. Thus, we should look for this by trial and error and gradually build upon our existing models. Meanwhile, we should keep using our models for purposes of prediction and experiments, because the fact that our models are unfinished does not imply that they are not useful.

\section{I.2 Theory, data or both, what kind of data and which theories?}

Similar to the previous discussion, the trade-off between using theories or empirical data is not a matter of one or the other. A balance should be found between the two. Historically, most ABMs were very stylised and mostly theory-based, followed by a trend of adding more empirical data to inform behavioural rules. Pure theoretic models provide a solid base for describing and predicting human behaviour, and therefore they are easy to generalise. However, theoretical models are poorly equipped in dealing with heterogeneous agents with diverse preferences, which is one of the strengths of ABM. Moreover, theories need to be parameterised in order to formalise decision rules, even the most widely used and well established ones. Therefore, you need at least some form of empirical data to validate and parameterise the decision rules. On the other hand, ABMs that are purely based on empirical data are criticised for being too ad-hoc and not generalizable. In my thesis I start with a strongly theoretical approach of modelling human behaviour, and I gradually shift towards a more empirical approach. This was driven by the desire to better understand, from the bottom up, the micro-level behaviour that underlies the observed patterns in the housing market. In my thesis I have found that the empirical data helped me to better capture the details of micro-level preferences of individuals. Moreover, I found in chapter 5 that the theoretical framework that best explains how humans respond to flood risk may change over time if floods become more of an issue in terms of frequency and/or severity. Consequently, adding empirical observations to the framework (?) benefited the selection of a theory and thus the prediction value of the model. This finding is supported by Sun et al. (2016), who argue that $A B M s$ used for purposes of prediction also rely more on empirical data than models that are merely used for understanding system dynamics or for theory building. 
Throughout this thesis I have used multiple behavioural theories and assessed their impact on the market. The reason I used theories is that they provide better grounding and generalisability of the modelled behaviour than ad-hoc behavioural rules based on empirical data In chapters 3 and 4 we saw that the choice of a theory matters a lot in the simulated outcome. Thus the choice of a theory and how it is implemented is an important one in the prediction outcome. In this regard I went beyond the borders of a single discipline. Some theories, in particular from microeconomics, have only few variables and already consists of clear equations that can be implemented in a model, while other theories from sociology and psychology have more variables and more degrees of freedom in how they are operationalised in a model. We have seen in chapter 3 that the selection of a theory could not be done purely on thebasis of simulation output, nor should simplicity be an argument to select one theory over another when their aggregated outcome differs so much. Therefore, I have used survey data in chapter 5 that gave me a more detailed insight into the decision making process at the individual level. This data highlighted that context matters a lot in which theory best describes human behaviour (protection motivation theory for protective measures such as insuring, and affect heuristics for the decision to evade hazard areas). I have found that empirical data can support the choice of a theory and facilitate the parameterisation of variables of a theory when it is operationalised in the model. There should be some prior reasoning behind the theories that are taken into consideration, but the selection and implementation of a theory can be supported well with empirical data.

As a contribution to the discussion what kind of empirical data should be used, I have found in my thesis that micro level data is essential to support the selection and parameterisation of a theoretical model. Moreover, the strength of micro level data for $A B M$ in particular is that it supports the modelling of heterogeneity at the level of the individual. In chapter 3 we have seen that some macro level patterns in the system, in particular the weak ones, can easily be distorted by statistical biases and a failure to distinguish signal from noise in the simulated output. Also, in empirical datasets, the aggregated patterns are not necessarily an obvious result from behaviour at the micro level. The patterns may strongly differ per case study when subtle patterns in society are masked by noise. Systemic patterns in CHANS are the result of many complex interactions that are sometimes hard to trace back. Hence, the parameterisation of micro-level behavioural theories can be very challenging when you cannot even rely on how we explain patterns that emerge in empirical as well as in simulated systems. In ABM we explicitly focus formulation of behavioural rules at the micro level to see what patterns can emerge in a system. Therefore, it would be inefficient to rely on macro-level data to validate behavioural rules on the 
micro level, even if it is combined with well-established behavioural theories. Furthermore, when exploring future scenarios in CHANS that are subject to regime shifts, such aggregate data are often non-existent. Validation based on historical pathways at the macro level is therefore insufficient for two reasons. One, because history is also just a single possible outcome; the historical pathways of complex systems may have been very different as a consequence of even the slightest disturbance. Two, because future environmental conditions in CHANS will most probably be very different, for example as a consequence of unprecedented storm severity. In chapter 5 and 6 we have seen that the consequence of such trends may already be visible on a small scale, and that such patterns at the micro level can easily be scaled up in an ABM in order to explore how the system will behave in a scenario where high impact floods happen frequently.

Human behaviour in ABM is strengthened when a modeller is open to multiple microlevel behavioural theories from various fields within the social sciences, and when these theories are parameterised and supported by empirical micro-level data such as surveys, interviews and lab experiments. Therefore, I strongly recommend to modellers to explore behavioural theories outside one's field, and make detailed disaggregate data collection common practice when modelling human behaviour in ABMs of CHANS, in particular when these models are used for purposes of prediction.

\section{I.3 Steps in modelling human behaviour in CHANS}

As a last methodological contribution resulting from this thesis I would like to propose a few steps that in my view every modeller should consider when addressing human behaviour in CHANS (Fig 7.I). In particular when working on a model that is designed to address the grand scientific challenges of the Anthropocene.

\section{Start with building a conceptual model}

One must understand the system well, before one can even start making modelling decisions. In this part the modeller must develop a mechanistic understanding of the system, i.e.: which aspects of human behaviour at the micro level could explain systemic patterns of CHANS at the macro level? At this stage it is not yet about formulating theories, but about building a conceptual understanding of how humans impact the natural system and vice versa. This inescapably involves the collection of empirical observations of some sort, either purely explorative or more in-depth. One cannot simply start modelling a system from behind a desk by studying theories and reading papers. Interviews and discussions with peers and experts may help to ensure none of the essential elements of people's behaviour in CHANS are missed. 


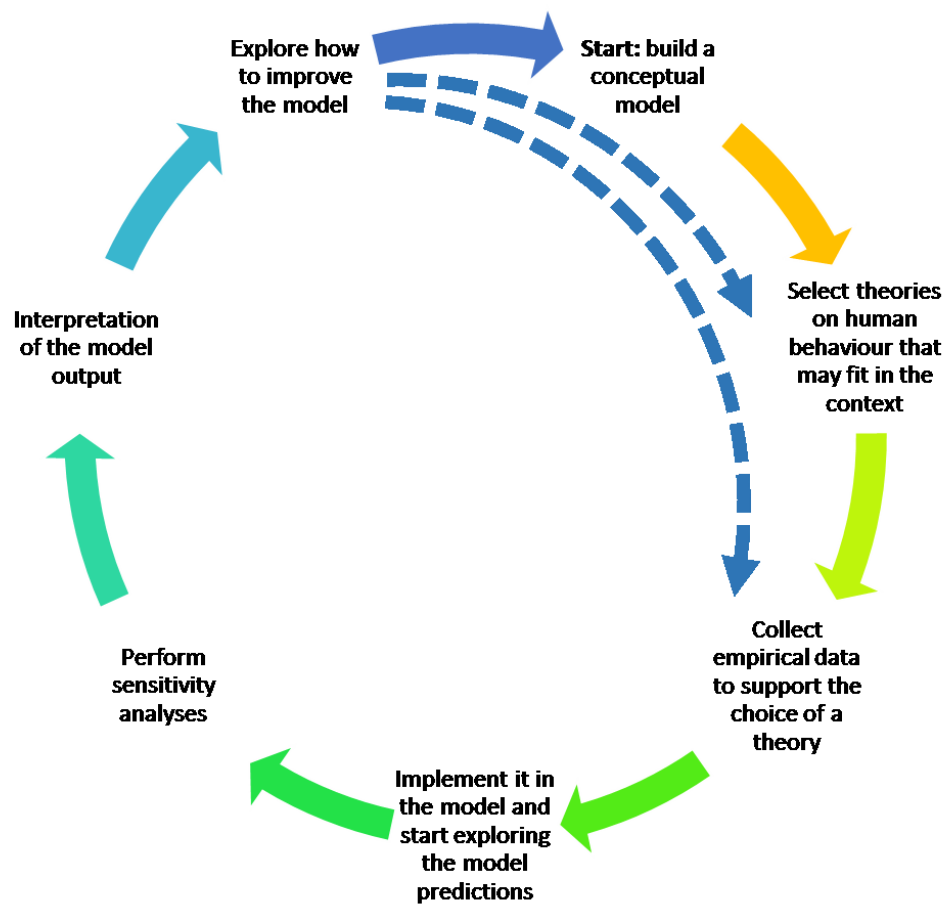

Figure 7.I. Steps to implement human behaviour in models of CHANS. Note that exploring how to improve the model may not necessarily lead to repeating all steps, as is shown by the short-cuts of the dashed arrows.

2. Select a number of theories of human behaviour that may fit in the context Once the main behavioural aspects of humans are highlighted in the system, it is time to gather theories that can explain human behaviour in the specific context, and that can predict people's choices when they are given various options. I suggest to go beyond one's disciplinary field. Theories from economics may provide the simplest model structure and therefore the best alternatives, but more complicated theories from sociology or psychology may fit better in the context of the decisions. At this stage it is not required to select only one theory. You can choose multiple theories from various fields, and use empirical data in a later stage to determine which theory fits best in the context of the decions.

3. Collect empirical data to support the definitive choice of a theory

The collection of empirical data should be done by taking all selected behavioural theories into account. This is relevant at the stage of designing the surveys, interviews or lab experiments, which should allow for validating which of the theories best fits 
human behaviour in the specific context. Moreover, the empirical data is a good way to kill two birds with one stone: selecting a theory and adding heterogeneity on the agent level. Hence, you must make sure that the data is collected in such a way that it can also serve as direct input source for modelling heterogeneous agents. Lastly, the empirical data should ideally represent a large sample so that the data can also be used for heuristic decision rules supported by Bayesian statistics.

4. Implement the data and theories in the model and start exploring the model predictions

This stage is all about coding. Most importantly, the modeller must make sure that the code is correct. Do the agents in the model behave in the way that they are expected to behave? Does their behaviour fit with how it is described in the theories? Does it also fit well with the empirical input data? Are there no other bugs in the code? Furthermore, at this stage it is important to explore a multitude of variables that represent the behaviour of the modelled system. The modeller should be aware that $A B M s$ produce a large number of variables that can be analysed (Lee et al. 2015). Be creative and think of which macro-level variables can best describe the main aspects of the system. This also depends on the focus of your research questions.

5. Perform sensitivity analyses

A sensitivity analysis is done to check how sensitive the aggregated outcome of the model predictions is to the behavioural assumptions at the micro level. This can be done by comparing the aggregate outcome of various theories, or by altering other assumptions in the model. Moreover, we can assess the models' sensitivity to uncertainty in the input data. Empirical data does not give a model that explains all behavioural variation in a population, and it should be considered how slightly different data could have changed the input parameterisation of human behaviour in the model, and how this has an impact on the results at the macro level.

6. Interpretation of the model output

How the model output should be interpreted is a combination of step 4 and 5 . The selected output variables give an overview of how a system behaves on the aspects that a researcher or policy maker is interested in. Here it is key to focus on general patterns in the system (Grimm et al. 2005), and not necessarily at the micro level details and numbers. The sensitivity analysis gives insight into how much care should be taken in the interpretation of the model output. Naturally, if the output patterns are very sensitive to the input assumptions, the interpretation must be handled with 
care. Conversely, when the simulated macro-level patterns are consistent no matter the uncertainty, these can be relied on with more confidence.

7. Make stepwise improvements to the model

Focus on those parts of the model where the output is most sensitive to the input assumptions. It could be worthwhile to work out the mechanistic framework in more detail and collect more data where needed. The use of Bayesian statistics instead of the frequentist approach helps because one can simply add new data that builds on the existing knowledge, and hence the data collection is constructive and not repetitive. At this point some of the previous steps can be repeated to make iterative improvements of the model (Jakeman et al. 2006). It should be kept in mind that a model is never finished. Nevertheless we should continue using our existing models for policy recommendations since as I have shown they have value, even though we should continue improving them.

\subsection{Agenda for future research}

My thesis has provided insights in how the modelling of human behaviour in CHANS can be improved, so that they can be of more effective use when addressing the grand scientific challenges of the Anthropocene. I conclude this thesis by sharing my thoughts on potentially interesting methodological improvements and good modelling practices that could be explored further in the future.

First, it will be relevant to know if my conclusions and recommendations hold in other case studies of modelling human behaviour in CHANS. My thesis is based on a single case study of human decisions in the housing market that is at increased risk of flooding due to climate change. The steps that I followed to improve the formalisation of human behaviour in a mathematical simulation model translated in the seven iterative steps suggested in Fig. 7.I that other modellers can follow. It would enhance our understanding by applying these steps to various models of CHANS to see if this framework does indeed contribute to good modelling practices and a better representation of human behaviour in our simulation models. Others may need to build further on the framework so that it can be generalised for all models of CHANS.

Secondly, future work should focus on connecting models from multiple disciplines. My thesis is focused mostly on the human aspect of CHANS, with little attention to the feedback mechanisms of how residential location choice affects the natural flow of water. This case study is illustrative of how human behaviour and the societal 
impacts of their behaviour can be modelled. Yet, in other models of CHANS, we may need more focus connecting different types of models. We should determine how models of human systems can be connected with more detailed models of natural systems (e.g. hydrology, ecology and geomorphology).

Thirdly and related is the focus on collaboration with researchers from multiple disciplines. In my thesis I have worked on the model mostly on my own, whereas I use models and theories from multiple disciplines. Hence, it is way more efficient and probably more effecitive to combine the work of experts from various fields. The need for more collaboration between researchers from different fields in modelling of CHANS is obvious and has been highlighted by other researchers (Ramanath and Gilbert 2004; Matthews et al. 2007; Verburg et al. 2016). Yet, there are still gaps in terms of how to do this which need to be filled in. For example: how to strengthen communication across scientific disciplines? What conditions are needed at the university to foster collaboration between scientists from various disciplines? How to ensure that qualitative researchers can provide the right input for modellers so that knowledge can easily be implemented in a model? How to deal with different versions of a large model where various researches work on simultaneously (Payette 2018)? And how to deal with computational phobia of social sciences, and with social sciences phobia of natural scientists?

Lastly, we must develop a way to make our complex (agent-based) models useful and easy to handle for policy makers. Scientists and policy makers have a shared responsibility in ensuring that the right decisions are made in order to secure the existence and well-being of our species in the future. Hence, modellers are responsible for ensuring that models of CHANS can easily be used and interpreted by policy makers. This involves giving transparency about how the models are designed, without drowning the lay person with the complex details of our models. The right balance must be found in giving sufficient transparency and leaving parts of the models a black box. We must think about user-friendly interfaces that allow policy makers to play with our models and design their own experiments, and think of good ways to visualise model outputs. The output must be a good balance between detail and abstractness, which should provide the right clarity and leave little room for false interpretation. Only then we can properly support policy makers to make informed decisions about the future of our planet. 


\section{BIBLIOGRAPHY}

Adamowicz, W., Louviere, J., \& Williams, M. (1994). Combining revealed and stated preference methods for valuing environmental amenities. Journal of environmental economics and management, 26(3), 27I-292.

Adamowicz, W., Swait, J., Boxall, P., Louviere, J., \& Williams, M. (1997). Perceptions versus objective measures of environmental quality in combined revealed and stated preference models of environmental valuation. Journal of environmental economics and management, 32(I), 65-84.

Adger, W. N., Arnell, N. W., \& Tompkins, E. L. (2005). Successful adaptation to climate change across scales. Global environmental change, I5(2), 77-86.

Adger, W. N., Hughes, T. P., Folke, C., Carpenter, S. R., \& Rockström, J. (2005). Social-ecological resilience to coastal disasters. Science, 309(5737), I036-I039.

Adger, W. N., Arnell, N. W., Black, R., Dercon, S., Geddes, A., \& Thomas, D. S. (2015). Focus on environmental risks and migration: causes and consequences. Environmental Research Letters, 10(6), 06020I.

Alemi MH, Azari AS, Nielsen DR (1988) Kriging and univariate modeling of a spatially correlated data. Soil Technology I: I33-147

Alexander, K. S., Ryan, A., \& Measham, T. G. (2012). Managed retreat of coastal communities: understanding responses to projected sea level rise. Journal of Environmental Planning and Management, 55(4), 409-433.

Amundson, R., Berhe, A. A., Hopmans, J. W., Olson, C., Sztein, A. E., \& Sparks, D. L. (20I5). Soil and human security in the 2 I st century. Science, 348(6235), I26I07I.

An, L. (20I2). Modeling human decisions in coupled human and natural systems: review of agent-based models. Ecological Modelling, 229, 25-36.

An, L., \& López-Carr, D. (20I2). Understanding human decisions in coupled natural and human systems. Ecological Modelling, (229), I-4.

Arthur, W. B. (1999). Complexity and the economy. science, 284(54I I), I07-I09.

Atreya, A., Ferreira, S., \& Kriesel, W. (2013). Forgetting the flood? An analysis of the flood risk discount over time. Land Economics, 89(4), 577-596.

Atreya, A., \& Czajkowski, J. (2016). Graduated flood risks and property prices in Galveston County. Real Estate Economics. 
Axtell, R. (2005). The complexity of exchange. The Economic Journal, I I5(504), FI93-F2I0.

Bagstad, K. J., Stapleton, K., \& D'Agostino, J. R. (2007). Taxes, subsidies, and insurance as drivers of United States coastal development. Ecological Economics, 63(2-3), 285298.

Barberis, N. C. (20I3). Thirty years of prospect theory in economics: A review and assessment. Journal of Economic Perspectives, 27(I), I73-96.

Basu S, Thibodeau TG (1998) Analysis of Spatial Autocorrelation in House Prices. Journal of Real Estate Finance and Economics I7(I): 6I-85

Bell, A. R. (20|7). Informing decisions in agent-based models-A mobile update. Environmental Modelling \& Software, 93, 310-321.

Beltrán, A., Maddison, D., \& Elliott, R. J. (2018). Is flood risk capitalised into property values?. Ecological Economics, I46, 668-685.

Bin $O$ (2004) A prediction comparison of housing sales prices by parametric versus semi-parametric regressions. Journal of Housing Economics 13: 68-84

Bin, O., \& Polasky, S. (2004). Effects of flood hazards on property values: evidence before and after Hurricane Floyd. Land Economics, 80(4), 490-500.

Bin, O., Kruse, J. B., \& Landry, C. E. (2008a). Flood hazards, insurance rates, and amenities: Evidence from the coastal housing market. Journal of Risk and Insurance, 75(I), 63-82.

Bin, O., Crawford, T. W., Kruse, J. B., \& Landry, C. E. (2008b). Viewscapes and flood hazard: Coastal housing market response to amenities and risk. Land economics, 84(3), 434-448.

Bin, O., \& Landry, C. E. (20I3). Changes in implicit flood risk premiums: Empirical evidence from the housing market. Journal of Environmental Economics and management, 65(3), 36I-376.

Black, R., Kniveton, D., \& Schmidt-Verkerk, K. (20I I). Migration and climate change: towards an integrated assessment of sensitivity. Environment and Planning A, 43(2), $43 \mathrm{I}-450$. 
Black, R., Arnell, N. W., Adger, W. N., Thomas, D., \& Geddes, A. (20I3). Migration, immobility and displacement outcomes following extreme events. Environmental Science \& Policy, 27, S32-S43.

Board, M. A. (2007). National security and the threat of climate change. Alexandria: CNA Corporation.

Bockstael, N. E. (1996). Modeling economics and ecology: the importance of a spatial perspective. American Journal of Agricultural Economics, 78(5), I I68-I I80.

Bokhari, S., \& Geltner, D. (20II). Loss aversion and anchoring in commercial real estate pricing: Empirical evidence and price index implications. Real Estate Economics, 39(4), 635-670.

Bonabeau, E. (2002). Agent-based modeling: Methods and techniques for simulating human systems. Proceedings of the National Academy of Sciences, 99(suppl 3), 7280 7287.

Bostic R, Gabriel S, Painter G (2005) Housing wealth, financial wealth, and consumption: new evidence from micro data. Regional Science and Urban Economics 39(I): 79-89

Botzen, W. J. W., Aerts, J. C. J. H., \& Van Den Bergh, J. C. J. M. (2009). Dependence of flood risk perceptions on socioeconomic and objective risk factors. Water Resources Research, 45(10).

Botzen, W. J., \& Van Den Bergh, J. C. (20I2). Monetary valuation of insurance against flood risk under climate change. International Economic Review, 53(3), 1005-1026.

Bourassa SC, Cantoni E, Hoesli M (2007) Spatial Dependence, Housing Submarkets and House Price Prediction. Journal of Real Estate Finance and Economics 35: I43160

Brookshire, D. S., Thayer, M. A., Tschirhart, J., \& Schulze, W. D. (1985). A test of the expected utility model: Evidence from earthquake risks. journal of Political Economy, 93(2), 369-389.

Brown, D. G., \& Robinson, D. T. (2006). Effects of heterogeneity in residential preferences on an agent-based model of urban sprawl. Ecology and society, I I(I).

Browne, M. J., \& Hoyt, R. E. (2000). The demand for flood insurance: empirical evidence. Journal of risk and uncertainty, 20(3), 29I-306. 
Bubeck, P., Botzen, W. J., \& Aerts, J. C. (20I2). A review of risk perceptions and other factors that influence flood mitigation behavior. Risk Analysis: An International Journal, 32(9), |48|-|495.

Bubeck, P., Botzen, W. J., Kreibich, H., \& Aerts, J. C. (20I3). Detailed insights into the influence of flood-coping appraisals on mitigation behaviour. Global Environmental Change, 23(5), I327-1338.

Buchmann, C. M., Grossmann, K., \& Schwarz, N. (2016). How agent heterogeneity, model structure and input data determine the performance of an empirical ABM-a real-world case study on residential mobility. Environmental Modelling \& Software, 75, 77-93.

Burningham, K., Fielding, J., \& Thrush, D. (2008). 'It'll never happen to me': understanding public awareness of local flood risk. Disasters, 32(2), 216-238.

Cameron, T. A., Poe, G. L., Ethier, R. G., \& Schulze, W. D. (2002). Alternative nonmarket value-elicitation methods: are the underlying preferences the same?. Journal of Environmental Economics and Management, 44(3), 39l-425.

Carpenter, S. R., \& Kitchell, J. F. (Eds.). (1996). The trophic cascade in lakes. Cambridge University Press.

Case B, Clapp J, Dubin R, Rodriguez M (2004) Modelling Spatial and Temporal House Price Patterns: A Comparison of Four Models. Journal of Real Estate Finance and Economics 29(2): |67-19|

Chivers, J., \& Flores, N. E. (2002). Market failure in information: the national flood insurance program. Land Economics, 78(4), 5I5-52I.

Collen, H., \& Hoekstra, J. (200I). Values as determinants of preferences for housing attributes. Journal of Housing and the Built Environment, 16(3-4), 285-306.

Congressional Budget Office (2017). The National Flood Insurance Program: Financial Soundness and Affordability

Crutzen, P. J. (2002). Geology of mankind. Nature, 4I5(6867), 23.

Daniel, V. E., Florax, R. J., \& Rietveld, P. (2009). Flooding risk and housing values: An economic assessment of environmental hazard. Ecological Economics, 69(2), 355365. 
De Blaeij, A., Florax, R. J., Rietveld, P., \& Verhoef, E. (2003). The value of statistical life in road safety: a meta-analysis. Accident Analysis \& Prevention, 35(6), 973-986.

de Koning, K., Filatova, T., \& Bin, O. (2017). Bridging the Gap Between Revealed and Stated Preferences in Flood-prone Housing Markets. Ecological economics, 136, I13.

de Koning, K., Filatova, T., \& Bin, O. (2018). Improved Methods for Predicting Property Prices in Hazard Prone Dynamic Markets. Environmental and resource economics, 69(2), 247-263.

de Koning, K., Filatova, T. Bin, O., Need, A. (20/8). Evading or mitigating flooding: bottom-up drivers of urban resilience to climate change. Manuscript under review

de Sherbinin, A., Castro, M., Gemenne, F., Cernea, M. M., Adamo, S., Fearnside, P. M., ... \& Scudder, T. (20II). Preparing for resettlement associated with climate change. Science, 334(6055), 456-457.

Delhomme JP (1978) Kriging in the Hydrosciences. Advances in Water Resources I(5): 25 I-266

Dixon, L., N. Clancy, S. A. Seabury \& A. Overton (2006). The National Flood Insurance Program's Market Penetration Rate: Estimates and Policy Implications. Santa Monica, California, RAND Corporation. Availablae online: http://www.rand.org/pubs/technical_reports/TR300.html. Accessed: January 6, 2014.

Do AQ, Grudnitski G (1993) A Neural Network Analysis of the Effect of Age on Housing Values. Journal of Real Estate Research 8(2): 253-264

Donovan, G. H., Champ, P. A., \& Butry, D. T. (2007). Wildfire risk and housing prices: a case study from Colorado Springs. Land Economics, 83(2), 2I 7-233.

Dronkers, J., Gilbert, J.T.E., Butler, L.W., Carey, J.J., Campbell, J., James, E., McKenzie, C., Misdorp, R., Quin, N., Ries, K.L., Schroder, P.C., Spradley, J.R., Titus, J.G., Vallianos, L., von Dadelszen, J. (1990). Strategies for Adaptation to Sea Level Rise. Report of the IPCC Coastal Zone Management Subgroup: Intergovernmental Panel on Climate Change. Geneva: Intergovernmental Panel on Climate Change

Dubbelboer, J., Nikolic, I., Jenkins, K., \& Hall, J. (2017). An agent-based model of flood risk and insurance. Journal of Artificial Societies and Social Simulation, 20(I).

Dubin, R. A. (1992). Spatial autocorrelation and neighborhood quality. Regional science and urban economics, 22(3), 433-452. 
Bibliography

Dubin RA (1999) Predicting House Prices Using Multiple Listings Data. Journal of Real Estate Finance and Economics 17(1): 35-59

Duinen, R. V., Filatova, T., Geurts, P., \& Veen, A. V. D. (2015). Empirical analysis of farmers' drought risk perception: Objective factors, personal circumstances, and social influence. Risk analysis, 35(4), 74I-755.

Dutta D, Herath S, Musiake K (2003) A mathematical model for flood loss estimation. Journal of Hydrology 277: 24-49

Earnhart, D. (1998). Combining revealed and stated data to examine decisions of housing location: Discrete-Choice Hedonic and Conjoint Analysis. University of Kansas.

Earnhart, D. (200I). Combining revealed and stated preference methods to value environmental amenities at residential locations. Land economics, 77(1), 12-29.

Ellison, A. M. (2004). Bayesian inference in ecology. Ecology letters, 7(6), 509-520.

Ettema, D. (20II). A multi-agent model of urban processes: Modelling relocation processes and price setting in housing markets. Computers, environment and urban systems, 35(I), I-II.

Faist, T., \& Schade, J. (20I3). Disentangling migration and climate change. Springer.

FAO, ITPS (2015). Status of the World's Soil Resources (SWSR)-Main Report. Food and Agriculture Organization of the United Nations and Intergovernmental Technical Panel on Soils, Rome, Italy, 650.

Farber S (1987) The Value of Coastal Wetlands for Protection of Property against Hurricane Wind Damage. Journal of Environmental Economics and Management 14: |43-|5|

Farmer, J. D., \& Foley, D. (2009). The economy needs agent-based modelling. Nature, 460(7256), 685.

Farmer, J. D., Hepburn, C., Mealy, P., \& Teytelboym, A. (20I5). A third wave in the economics of climate change. Environmental and Resource Economics, 62(2), 329. 357.

FEMA (2015). FEMA P-1037, Reducing Flood Risk to Residential Buildings That Cannot Be Elevated, https://www.fema.gov/media-library/assets/documents//09669

FEMA (2018) FEMA Flood Map Service Center, https://msc.fema.gov/portal/home 
Filatova, T., Parker, D. C., \& van der Veen, A. (20II). The implications of skewed risk perception for a Dutch coastal land market: insights from an agent-based computational economics model. Agricultural and Resource Economics Review, 40(3), 405-423.

Filatova, T. (20I5). Empirical agent-based land market: Integrating adaptive economic behavior in urban land-use models. Computers, environment and urban systems, 54, 397-4I3.

Filatova, T., Polhill, J. G., \& van Ewijk, S. (20/6). Regime shifts in coupled socioenvironmental systems: review of modelling challenges and approaches. Environmental modelling \& software, 75, 333-347.

Finucane, M. L., Alhakami, A., Slovic, P., \& Johnson, S. M. (2000). The affect heuristic in judgments of risks and benefits. Journal of behavioral decision making, I3(I), I- I7.

Florax, R. J., Travisi, C. M., \& Nijkamp, P. (2005). A meta-analysis of the willingness to pay for reductions in pesticide risk exposure. European Review of Agricultural Economics, 32(4), 44I-467.

Folke, C. (2006). Resilience: The emergence of a perspective for social-ecological systems analyses. Global environmental change, 16(3), 253-267.

Frame, D. E. (1998). Housing, natural hazards, and insurance. Journal of Urban Economics, 44(I), 93-109.

Freeman III, A. M., Herriges, J. A., \& Kling, C. L. (20I4). The measurement of environmental and resource values: theory and methods. Routledge.

Gamper CD, Thöni M, Weck-Hannemann H (2006) A conceptual approach to the use of Cost Benefit and Multi Criteria Analysis in natural hazard management. Natural Hazards and Earth System Sciences 6: 293-302

Ge, Y., Peacock, W. G., \& Lindell, M. K. (20II). Florida households' expected responses to hurricane hazard mitigation incentives. Risk Analysis: An International Journal, 3 I(10), I676-169I.

Gigerenzer, G., \& Goldstein, D. G. (1996). Reasoning the fast and frugal way: models of bounded rationality. Psychological review, 103(4), 650.

Gigerenzer, G., \& Selten, R. (Eds.). (2002). Bounded rationality: The adaptive toolbox. MIT press. 
Gigerenzer, G., \& Gaissmaier, W. (20II). Heuristic decision making. Annual review of psychology, 62, 45 I-482.

Gilbert, N., \& Terna, P. (2000). How to build and use agent-based models in social science. Mind \& Society, I ( I), 57-72.

Gilbert, N., Hawksworth, J. C., \& Swinney, P. A. (2009, March). An Agent-Based Model of the English Housing Market. In AAAI Spring Symposium: Technosocial Predictive Analytics (Pp. 30-35).

Goodman AC Thibodeau TG (1995) Age-Related Heteroskedasticity in Hedonic House Price Equations. Journal of Housing Research 6(I): 25-42

Gotts, N. M., van Voorn, G. A., Polhill, J. G., de Jong, E., Edmonds, B., Hofstede, G. J., \& Meyer, R. (20I8). Agent-based modelling of socio-ecological systems: Models, projects and ontologies. Ecological Complexity.

Grimm, V., Revilla, E., Berger, U., Jeltsch, F., Mooij, W. M., Railsback, S. F., ... \& DeAngelis, D. L. (2005). Pattern-oriented modeling of agent-based complex systems: lessons from ecology. science, $310(5750)$, 987-991.

Groeneveld, J., Müller, B., Buchmann, C. M., Dressler, G., Guo, C., Hase, N., ... \& Liebelt, V. (20I7). Theoretical foundations of human decision-making in agent-based land use models-A review. Environmental modelling \& software, 87, 39-48.

Grothmann, T., \& Reusswig, F. (2006). People at risk of flooding: why some residents take precautionary action while others do not. Natural hazards, 38(I-2), I0I-I20.

Haer, T., Botzen, W. W., de Moel, H., \& Aerts, J. C. (20I7). Integrating household risk mitigation behavior in flood risk analysis: an agent- based model approach. Risk Analysis, 37(10), 1977-1992.

Hall JW et al (2003) A methodology for national-scale flood risk assessment. Proceedings of the ICE-Water and Maritime Engineering I56(3): 235-247

Hall JW, Sayers PB, Dawson RJ (2005) National-scale Assessment of Current and Future Flood Risk in England and Wales. Natural Hazards 36: 147-I64

Hallegatte S (2006) A Cost-Benefit Analysis of the New Orleans Flood Protection System. AEI-Brookings Joint Center. Regulatory Analysis: 06-02

Hallstrom, D. G., \& Smith, V. K. (2005). Market responses to hurricanes. Journal of Environmental Economics and Management, 50(3), 54I-56I. 
Harrison, D., T. Smersh, G., \& Schwartz, A. (200I). Environmental determinants of housing prices: the impact of flood zone status. Journal of Real Estate Research, 2 I ( I 2), 3-20.

Hensher, D., Louviere, J., \& Swait, J. (1998). Combining sources of preference data. Journal of Econometrics, 89(I-2), 197-22I.

Hernandez-Stefanoni JL, Ponce-Hernandez R (2006) Mapping the spatial variability of plant diversity in a tropical forest: comparison of spatial interpolation methods. Environmental Monitoring and Assessment I 17: 307-334

Herstein, I. N., \& Milnor, J. (1953). An axiomatic approach to measurable utility. Econometrica, Journal of the Econometric Society, 29I-297.

Hirabayashi $Y$ et al (20I3) Global flood risk under climate change. Nature Climate Change 3: 816-82I

Ho, M. C., Shaw, D., Lin, S., \& Chiu, Y. C. (2008). How do disaster characteristics influence risk perception?. Risk Analysis: An International Journal, 28(3), 635-643.

Howson, C., \& Urbach, P. (2006). Scientific reasoning: the Bayesian approach. Open Court Publishing.

IPCC. (20I2) Managing the Risks of Extreme Events and Disasters to Advance Climate Change Adaptation (eds Field, C. B. et al.), Cambridge Univ. Press, Cambridge IPCC (2014) Climate Change 2014: Synthesis Report. Contribution of Working Groups I, II and III to the Fifth Assessment Report of the Intergovernmental Panel on Climate Change [Core Writing Team, R.K. Pachauri and L.A. Meyer (eds.)]. IPCC, Geneva, Switzerland, I5I PP.

IPBES (2018). Biodiversity and Nature's Contributions Continue Dangerous Decline, Scientists Warn. https://www.ipbes.net/news/media-release-updated-biodiversitynature\%E2\%80\%99s-contributions-continue-dangerous-decline-scientists\#_ftnrefI

Irwin, E. G. (2010). New directions for urban economic models of land use change: incorporating spatial dynamics and heterogeneity. Journal of Regional Science, 50(I), 65-91.

Jakeman, A. J., Letcher, R. A., \& Norton, J. P. (2006). Ten iterative steps in development and evaluation of environmental models. Environmental Modelling \& Software, 21 (5), 602-6I4. 
Janssen C, Söderberg B, Zhou J (200I) Robust estimation of hedonic models of price and income for investment property. Journal of Property Investment and Finance 19(4): 342-360

Janssen, M. A., \& Ostrom, E. (2006). Empirically based, agent-based models. Ecology and society, II(2).

Jongman, B., Ward, P.J., \& Aerts, J.C.J.H. (2012). Global exposure to river and coastal flooding: Long term trends and changes. Global Environmental Change, 22, 823-835

Kahneman, D., \& Tversky, A. (1979). Prospect theory: An analysis of decision under risk. Econometrica: Journal of the Econometric Society, 263-29I

Katapodi, M. C., Lee, K. A., Facione, N. C., \& Dodd, M. J. (2004). Predictors of perceived breast cancer risk and the relation between perceived risk and breast cancer screening: a meta-analytic review. Preventive medicine, 38(4), 388-402.

Kates, R. W., Travis, W. R., \& Wilbanks, T. J. (20I2). Transformational adaptation when incremental adaptations to climate change are insufficient. Proceedings of the National Academy of Sciences, 20 I I I552I.

Keenan, J. M., Hill, T., \& Gumber, A. (2018). Climate gentrification: from theory to empiricism in Miami-Dade County, Florida. Environmental Research Letters, 13(5), 054001 .

Kellens, W., Terpstra, T., \& De Maeyer, P. (20I3). Perception and communication of flood risks: a systematic review of empirical research. Risk Analysis: An International Journal, 33(I), 24-49.

Keller, C., Siegrist, M., \& Gutscher, H. (2006). The role of the affect and availability heuristics in risk communication. Risk analysis, 26(3), 631-639.

Kelly, R. A., Jakeman, A. J., Barreteau, O., Borsuk, M. E., ElSawah, S., Hamilton, S. H., ... \& van Delden, H. (2013). Selecting among five common modelling approaches for integrated environmental assessment and management. Environmental modelling \& software, $47,159-181$.

Kirman, A. P. (1992). Whom or what does the representative individual represent?. Journal of economic perspectives, 6(2), $117-136$.

Knuth, D., Kehl, D., Hulse, L., \& Schmidt, S. (20I4). Risk Perception, Experience, and Objective Risk: A Cross- National Study with European Emergency Survivors. Risk analysis, 34(7), I286-I 298. 
Koerth, J., Vafeidis, A. T., \& Hinkel, J. (2017). Household- level coastal adaptation and its drivers: A systematic case study review. Risk Analysis, 37(4), 629-646.

Kousky, C. (2010). Learning from extreme events: Risk perceptions after the flood. Land Economics, 86(3), 395-422.

Kousky, C., \& Michel- Kerjan, E. (2017). Examining flood insurance claims in the United States: Six key findings. Journal of Risk and Insurance, 84(3), 819-850.

Kriesel, W., \& Landry, C. (2004). Participation in the National Flood Insurance Program: An empirical analysis for coastal properties. Journal of Risk and Insurance, 7I (3), 405-420.

Kunreuther, H. C., \& Michel-Kerjan, E. O. (2007). Climate change, insurability of large-scale disasters and the emerging liability challenge (No. wI282I). National Bureau of Economic Research.

Kuntz M, Helbich M (20I4) Geostatistical mapping of real estate prices: an empirical comparison of kriging and cokriging. International Journal of Geographical Information Science 28(9): 1904-1921

Laciana, C., Weber, E., Bert, F., Podestá, G., González, X., \& Letson, D. (2006). Objective functions in agricultural decision-making: A comparison of the effects of expected utility, regret-adjusted expected utility, and prospect theory maximization. In Working Papers Series, Paper WP-06-0I, Columbia University.

Laurice J, Bhattacharya R (2005) Prediction Performance of a Hedonic Pricing Model for Housing. Appraisal Journal 73(2)

Leadley, P. (2010). Biodiversity scenarios: projections of 21 st century change in biodiversity, and associated ecosystem services: a technical report for the global biodiversity outlook 3 (No. 50). UNEP/Earthprint.

Lee, J. S., Filatova, T., Ligmann-Zielinska, A., Hassani-Mahmooei, B., Stonedahl, F., Lorscheid, I., ... \& Parker, D. C. (20I5). The complexities of agent-based modeling output analysis. Journal of Artificial Societies and Social Simulation, 18(4), 4.

Lindell, M. K., \& Hwang, S. N. (2008). Households' perceived personal risk and responses in a multihazard environment. Risk Analysis: An International Journal, 28(2), 539-556. 
List, J. A., \& Gallet, C. A. (200I). What experimental protocol influence disparities between actual and hypothetical stated values?. Environmental and Resource Economics, 20(3), 24I-254.

Liu, J., Dietz, T., Carpenter, S. R., Alberti, M., Folke, C., Moran, E., ... \& Ostrom, E. (2007). Complexity of coupled human and natural systems. science, 3 I7(5844), I 5 I31516.

Ludy, J., \& Kondolf, G. M. (20I2). Flood risk perception in lands "protected” by 100 year levees. Natural hazards, 6I(2), 829-842.

MacDonald, D. N., Murdoch, J. C., \& White, H. L. (1987). Uncertain hazards, insurance, and consumer choice: Evidence from housing markets. Land Economics, 63(4), 36I-37I.

MacDonald, D. N., White, H. L., Taube, P. M., \& Huth, W. L. (1990). Flood hazard pricing and insurance premium differentials: evidence from the housing market. Journal of Risk and Insurance, 654-663.

Magliocca, N., McConnell, V., Walls, M., \& Safirova, E. (20I2). Zoning on the urban fringe: Results from a new approach to modeling land and housing markets. Regional Science and Urban Economics, 42(I-2), 198-210.

Magliocca, N. R., Brown, D. G., \& Ellis, E. C. (2013). Exploring agricultural livelihood transitions with an agent-based virtual laboratory: global forces to local decisionmaking. PLoS One, 8(9), e7324I.

Magliocca, N., McConnell, V., \& Walls, M. (20I5). Exploring sprawl: Results from an economic agent-based model of land and housing markets. Ecological Economics, II3, II4-I 25 .

Matthews, R. B., Gilbert, N. G., Roach, A., Polhill, J. G., \& Gotts, N. M. (2007). Agentbased land-use models: a review of applications. Landscape Ecology, 22(10), 1447 1459.

McCluskey, J. J., \& Rausser, G. C. (200I). Estimation of perceived risk and its effect on property values. Land Economics, 77(1), 42-55.

McNamara, D. E., \& Keeler, A. (2013). A coupled physical and economic model of the response of coastal real estate to climate risk. Nature Climate Change, 3(6), 559.

Meen, G. (2016). Spatial housing economics: A survey. 
Merz B et al (2010) Assessment of economic flood damage. Natural Hazards and Earth System Sciences 10: 1697-1724

Michel- Kerjan, E. O., \& Kousky, C. (2010). Come rain or shine: Evidence on flood insurance purchases in Florida. Journal of Risk and Insurance, 77(2), 369-397.

Militino AF, Ugarte MD, García-Reinaldos L (2004) Alternative Models for Describing Spatial Dependence among Dwelling Selling Prices. Journal of Real Estate Finance and Economics 29(2): 193-209

Milner-Gulland, E. J. (20/2). Interactions between human behaviour and ecological systems. Philosophical Transactions of the Royal Society B: Biological Sciences, 367(I586), 270-278.

Mitchell, R. C., \& Carson, R. T. (20I3). Using surveys to value public goods: the contingent valuation method. Rff Press.

Morgenstern, O., \& Von Neumann, J. (1953). Theory of games and economic behavior. Princeton university press.

Muelder, H., \& Filatova, T. (2018). One Theory-Many Formalizations: Testing Different Code Implementations of the Theory of Planned Behaviour in Energy Agent-Based Models. Journal of Artificial Societies \& Social Simulation, 2 I (4).

Mueller, M. G., \& de Haan, P. (2009). How much do incentives affect car purchase? Agent-based microsimulation of consumer choice of new cars-Part I: Model structure, simulation of bounded rationality, and model validation. Energy Policy, 37(3), 1072-1082.

Müller, B., Bohn, F., Dreßler, G., Groeneveld, J., Klassert, C., Martin, R., ... \& Schwarz, N. (20/3). Describing human decisions in agent-based models-ODD+ $D$, an extension of the ODD protocol. Environmental Modelling \& Software, 48, 37-48.

Müller-Hansen, F., Schlüter, M., Mäs, M., Donges, J. F., Kolb, J. J., Thonicke, K., \& Heitzig, J. (20I7). Towards representing human behavior and decision making in Earth system models-an overview of techniques and approaches. Earth System Dynamics, 8(4), 977-1007.

Murdoch, J. C., Singh, H., \& Thayer, M. (1993). The impact of natural hazards on housing values: the Loma Prieta earthquake. Real Estate Economics, 2 I (2), I67-I84. 
Murphy, J. J., Allen, P. G., Stevens, T. H., \& Weatherhead, D. (2005). A meta-analysis of hypothetical bias in stated preference valuation. Environmental and Resource Economics, 30(3), 313-325.

National Hurricane Centre (2018). Costliest U.S. tropical cyclones tables updated. https://www.nhc.noaa.gov/news/UpdatedCostliest.pdf

National Weather Service. Hydrologic Information Center - Flood Loss Data. http://www.nws.noaa.gov/hic. - visited 20/9/2018

Nguyen N, Cipps A (200I) Predicting Housing Value: A Comparison of Multiple Regression Analysis and Artificial Neural Networks 22(3): 313-336

Nicholls, R. J. (2004). Coastal flooding and wetland loss in the 2 I st century: changes under the SRES climate and socio-economic scenarios. Global Environmental Change, I4(I), 69-86.

Nolan, J., Parker, D., Van Kooten, G. C., \& Berger, T. (2009). An overview of computational modeling in agricultural and resource economics. Canadian Journal of Agricultural Economics/Revue canadienne d'agroeconomie, 57(4), 4I7-429.

Nordhaus, W. D. (2018) Prize Lecture. NobelPrize.org. Nobel Media AB 20I8. Tue. 18 Dec 2018. https://www.nobelprize.org/prizes/economicsciences/2018/nordhaus/lecture/

Nyce, C., Dumm, R. E., Sirmans, G. S., \& Smersh, G. (2015). The capitalization of insurance premiums in house prices. Journal of Risk and Insurance, 82(4), 89I-919.

Oliveri E, Santoro M (2000) Estimation of urban structural flood damages: the case study of Palermo. Urban Water 2: 223-234

Osberghaus, D. (2017). The effect of flood experience on household mitigationEvidence from longitudinal and insurance data. Global environmental change, 43, I26136.

O’Sullivan, D., Evans, T., Manson, S., Metcalf, S., Ligmann-Zielinska, A., \& Bone, C. (2016). Strategic directions for agent-based modeling: avoiding the YAAWN syndrome. Journal of land use science, II(2), I77-I87

Pagourtzi E et al (2003) Real estate appraisal: a review of valuation methods. Journal of Property Investment and Finance 2 I (4): 383-40 I 
Parker, D. C., \& Filatova, T. (2008). A conceptual design for a bilateral agent-based land market with heterogeneous economic agents. Computers, Environment and Urban Systems, 32(6), 454-463.

Parker, D. C., Duke, J., \& Wu, J. (2014). An economic perspective on agent-based models of land use and land cover change. The Oxford Handbook of Land Economics, 402.

Payette, N. (2018). Collaborating like professionals: integrating NetLogo and GitHub. Social Simulations Conference 2018 Stockholm August $23^{\text {rd }} 2018$

Penning-Rowsell E et al (2005) The Benefits of Flood and Coastal Risk Management: A Handbook of Assessment Techniques. Middlesex university press

Penning-Rowsell, E., Sultana, S., \& Thompson, P. Population Movement in Response to Climate Related Hazards in Bangladesh: The 'Last Resort'(Government Office for Science, 20II).

Pollak, R. A. (1967). Additive von Neumann-Morgenstern utility functions. Econometrica, Journal of the Econometric Society, 485-494.

Poussin, J. K., Botzen, W. W., \& Aerts, J. C. (20I4). Factors of influence on flood damage mitigation behaviour by households. Environmental Science \& Policy, 40, 69-77.

Pryce, G., Chen, Y., \& Galster, G. (20II). The impact of floods on house prices: an imperfect information approach with myopia and amnesia. Housing Studies, 26(02), 259-279.

Quigley, J. M., \& Raphael, S. (2004). Is housing unaffordable? Why isn't it more affordable?. Journal of Economic Perspectives, I8(I), I9I-2I4.

Rai, V., \& Robinson, S. A. (20I5). Agent-based modeling of energy technology adoption: empirical integration of social, behavioral, economic, and environmental factors. Environmental Modelling \& Software, 70, 163-I77.

Railsback, S. F., \& Grimm, V. (20I I). Agent-based and individual-based modeling: a practical introduction. Princeton university press.

Ramanath, A. M., \& Gilbert, N. (2004). The design of participatory agent-based social simulations. Journal of Artificial Societies and Social Simulation, 7(4).

Rambaldi, A. N., Fletcher, C. S., Collins, K., \& McAllister, R. R. (2013). Housing shadow prices in an inundation-prone suburb. Urban Studies, 50(9), 1889-1905. 
Reuveny, R. (2007). Climate change-induced migration and violent conflict. Political geography, 26(6), 656-673.

Rey-Valette, H., Robert, S., \& Rulleau, B. (2019). Resistance to relocation in floodvulnerable coastal areas: a proposed composite index. Climate Policy, 19(2), 206218.

Richetin, J., Sengupta, A., Perugini, M., Adjali, I., Hurling, R., Greetham, D., \& Spence, M. (20I0). A micro-level simulation for the prediction of intention and behavior. Cognitive Systems Research, II(2), I8I-193.

Rosen, S. (1974). Hedonic prices and implicit markets: product differentiation in pure competition. Journal of political economy, 82(I), 34-55.

Scheffer, M., Carpenter, S., \& de Young, B. (2005). Cascading effects of overfishing marine systems. Trends in Ecology \& Evolution, 20(I I), 579-58I.

Scheffer, M., Bascompte, J., Brock, W. A., Brovkin, V., Carpenter, S. R., Dakos, V., ... \& Sugihara, G. (2009). Early-warning signals for critical transitions. Nature, 46 I (7260), 53.

Schlüter, M., Baeza, A., Dressler, G., Frank, K., Groeneveld, J., Jager, W., ... \& Schwarz, N. (2017). A framework for mapping and comparing behavioural theories in models of social-ecological systems. Ecological Economics, 131, 21-35.

Schulze, J., Müller, B., Groeneveld, J., \& Grimm, V. (20I7). Agent-based modelling of social-ecological systems: Achievements, challenges, and a way forward. Journal of Artificial Societies and Social Simulation, 20(2).

Selim H (2009) Determinants of house prices in Turkey: Hedonic regression versus artificial neural network. Expert Systems with Applications 36(2): 2843-2852

Sewell, T., Stephens, R. E., Dominey-Howes, D., Bruce, E., \& Perkins-Kirkpatrick, S. (2016). Disaster declarations associated with bushfires, floods and storms in New South Wales, Australia between 2004 and 20I4. Scientific reports, 6, 36369.

Simon, H. A. (1956). Rational choice and the structure of the environment. Psychological review, 63(2), 129.

Simon, H. A. (1997). Models of bounded rationality: Empirically grounded economic reason (Vol. 3). MIT press. 
Slovic, P., Finucane, M. L., Peters, E., \& MacGregor, D. G. (2004). Risk as analysis and risk as feelings: Some thoughts about affect, reason, risk, and rationality. Risk analysis, 24(2), 3 II -322.

Slovic, P., Peters, E., Finucane, M. L., \& MacGregor, D. G. (2005). Affect, risk, and decision making. Health psychology, 24(4S), S35.

Smajgl, A., Brown, D. G., Valbuena, D., \& Huigen, M. G. (20II). Empirical characterisation of agent behaviours in socio-ecological systems. Environmental Modelling \& Software, 26(7), 837-844.

Smajgl, A., \& Bohensky, E. (20|3). Behaviour and space in agent-based modelling: poverty patterns in East Kalimantan, Indonesia. Environmental modelling \& software, $45,8-14$.

Smith, V. K., \& Desvousges, W. H. (1988). Risk perception, learning, and individual behavior. American Journal of Agricultural Economics, 70(5), I I I3- I I 7.

Speyrer, J. F., \& Ragas, W. R. (1991). Housing prices and flood risk: an examination using spline regression. The Journal of Real Estate Finance and Economics, 4(4), 395407.

Statista (2016). http://www.statista.com/markets/4I I/topic/970/economy/

Steffen, W., Rockström, J., Richardson, K., Lenton, T. M., Folke, C., Liverman, D., ... \& Donges, J. F. (2018). Trajectories of the Earth System in the Anthropocene. Proceedings of the National Academy of Sciences, II 5(33), 8252-8259.

Stern, P. C. (1993). A second environmental science: human-environment interactions. Science, 260(5II6), I897-1899.

Stern, N., Peters, S., Bakhshi, V., Bowen, A., Cameron, C., Catovsky, S., ... \& Edmonson, N. (2006). Stern Review: The economics of climate change (Vol. 30, p. 2006). London: HM treasury.

Stern, N. (2008). The economics of climate change. American Economic Review, 98(2), I-37.

Stern, N. (2016). Economics: current climate models are grossly misleading. Nature News, 530(759I), 407. 
Sun, S., Parker, D. C., Huang, Q., Filatova, T., Robinson, D. T., Riolo, R. L., ... \& Brown, D. G. (20I4). Market impacts on land-use change: An agent-based experiment. Annals of the Association of American Geographers, 104(3), 460-484.

Sun, Z., Lorscheid, I., Millington, J. D., Lauf, S., Magliocca, N. R., Groeneveld, J., ... \& Buchmann, C. M. (20I6). Simple or complicated agent-based models? A complicated issue. Environmental Modelling \& Software, 86, 56-67.

Terpstra, T. (201 I). Emotions, trust, and perceived risk: Affective and cognitive routes to flood preparedness behavior. Risk analysis, 3 I (I0), I658-I675.

Tesfatsion, L. (2002). Agent-based computational economics: Growing economies from the bottom up. Artificial life, 8(I), 55-82.

Tesfatsion, L. (2006). Agent-based computational economics: A constructive approach to economic theory. Handbook of computational economics, 2, 83 I-880.

Tesfatsion, L., \& Judd, K. L. (2006). Handbook of Computational Economics Volume II: Agent-Based Computational Economics Elsevier BV.

Tesfatsion, L. (20I7). Modeling economic systems as locally-constructive sequential games. Journal of Economic Methodology, 24(4), 384-409.

Timmermans, H., Molin, E., \& Van Noortwijk, L. (1994). Housing choice processes: Stated versus revealed modelling approaches. Netherlands Journal of Housing and the Built Environment, 9(3), 215-227.

Treuer, G., Broad, K., \& Meyer, R. (2018). Using simulations to forecast homeowner response to sea level rise in South Florida: Will they stay or will they go?. Global Environmental Change, 48, I08-II8.

Trumbo, C., Meyer, M. A., Marlatt, H., Peek, L., \& Morrissey, B. (20I4). An assessment of change in risk perception and optimistic bias for hurricanes among Gulf Coast residents. Risk analysis, 34(6), 1013-1024.

Tversky, A., \& Kahneman, D. (1992). Advances in prospect theory: Cumulative representation of uncertainty. Journal of Risk and uncertainty, 5(4), 297-323.

UNISDR (20II) Global Assessment Report on Disaster Risk Reduction. GARII

US Census Bureau (2016). http://www.census.gov/hhes/www/cpstables/0320I2/ hhinc/toc.htm 
US Census Bureau (2017). Income and Poverty in the United States: 2016. https://www.census.gov/data/tables/20 I 7/demo/income-poverty/p60-259.html

Valbuena, D., Verburg, P. H., \& Bregt, A. K. (2008). A method to define a typology for agent-based analysis in regional land-use research. Agriculture, Ecosystems \& Environment, 128(I-2), 27-36.

van Duinen, R. (2015). Exploring farmers' drought adaptation behaviour; the role of risk perceptions, coping factors and social interactions.

van Duinen, R., Filatova, T., Jager, W., \& van der Veen, A. (2016). Going beyond perfect rationality: drought risk, economic choices and the influence of social networks. The Annals of Regional Science, 57(2-3), 335-369.

van Valkengoed, A. M., \& Steg, L. (2019). Meta-analyses of factors motivating climate change adaptation behaviour. Nature Climate Change, I.

Verburg, P. H., Dearing, J. A., Dyke, J. G., van der Leeuw, S., Seitzinger, S., Steffen, W., \& Syvitski, J. (2016). Methods and approaches to modelling the Anthropocene. Global Environmental Change, 39, 328-340.

Viscusi, W. K. (1985). A Bayesian perspective on biases in risk perception. Economics Letters, I7(I-2), 59-62.

Voltz M, Webster R (1990) A comparison of kriging, cubic splines and classification for predicting soil properties from sample information. Journal of Soil Science $4 \mathrm{I}$ (3): 473-490.

Von Neumann, J., \& Morgenstern, O. (2007). Theory of games and economic behavior (commemorative edition). Princeton university press.

Walker, G., \& Burningham, K. (20II). Flood risk, vulnerability and environmental justice: Evidence and evaluation of inequality in a UK context. Critical social policy, $3 \mathrm{I}(2), 2 \mid 6-240$.

Ward PJ et al (2014) Strong influence of El Niño Southern Oscillation on flood risk around the world. Proceedings of the National Academy of Sciences I I I (44): I 565915664

Wardman, M. (1988). A comparison of revealed preference and stated preference models of travel behaviour. Journal of transport economics and policy, 7I-9I. 
Webster R, Burgess TM (1983) Spatial variation in soil and the role of kriging. Agricultural Water Management 6: I I I-I22

Webster, P. J., Holland, G. J., Curry, J. A., \& Chang, H. R. (2005). Changes in tropical cyclone number, duration, and intensity in a warming environment. Science, 309(5742), 1844-1846.

Weinstein, N. D. (2000). Perceived probability, perceived severity, and healthprotective behavior. Health Psychology, 19(I), 65.

Weinstein, N. D., Kwitel, A., McCaul, K. D., Magnan, R. E., Gerrard, M., \& Gibbons, F. X. (2007). Risk perceptions: assessment and relationship to influenza vaccination. Health Psychology, 26(2), 146.

Whitehead, J. C., Pattanayak, S. K., Van Houtven, G. L., \& Gelso, B. R. (2008). Combining revealed and stated preference data to estimate the nonmarket value of ecological services: an assessment of the state of the science. Journal of Economic Surveys, 22(5), 872-908.

Whitmarsh, L. (2008). Are flood victims more concerned about climate change than other people? The role of direct experience in risk perception and behavioural response. Journal of risk research, I I(3), 35 I-374.

Willis, K. F., Natalier, K., \& Revie, M. (20I I). Understanding risk, choice and amenity in an urban area at risk of flooding. Housing Studies, 26(02), 225-239.

Windrum, P., Fagiolo, G., \& Moneta, A. (2007). Empirical validation of agent-based models: Alternatives and prospects. Journal of Artificial Societies and Social Simulation, 10(2), 8.

Winsemius, H.C., Aerts, J.C.J.H., van Beek, L.P.H., Bierkens, M.F.P., Bouwman, A., Jongman, B., Kwadijk, J.C.J., Ligtvoet, W., Lucas, P.L., van Vuuren, D.P., \& Ward, P.J. (2016). Global drivers of future river flood risk. Nature Climate Change, 6, 38I-385

Wong, P.P., I.J. Losada, J.-P. Gattuso, J. Hinkel, A. Khattabi, K.L. Mclnnes, Y. Saito, and A. Sallenger (20I4). Coastal systems and low-lying areas. In: Climate Change 20 I4: Impacts, Adaptation, and Vulnerability. Part A: Global and Sectoral Aspects. Contribution of Working Group II to the Fifth Assessment Report of the Intergovernmental Panel on Climate Change [Field, C.B., V.R. Barros, D.J. Dokken, K.J. Mach, M.D. Mastrandrea, T.E. Bilir, M. Chatterjee, K.L. Ebi, Y.O. Estrada, R.C. Genova, B. Girma, E.S. Kissel, A.N. Levy, S. MacCracken, P.R. Mastrandrea, and L.L. White (eds.)]. Cambridge University Press, Cambridge, United Kingdom and New York, NY, USA, 36 I-409 
Zaalberg, R., Midden, C., Meijnders, A., \& McCalley, T. (2009). Prevention, adaptation, and threat denial: Flooding experiences in the Netherlands. Risk Analysis: An International Journal, 29(12), I759-I778.

Zaninetti, J. M., \& Colten, C. E. (20/2). Shrinking New Orleans: Post-Katrina population adjustments. Urban Geography, 33(5), 675-699.

Zillow (2018). Data, Home Values, https://www.zillow.com/research/data/ - downloaded $13 / 3 / 2018$

Zinas, B. Z., \& Jusan, M. B. M. (20I2). Housing choice and preference: Theory and measurement. Procedia-Social and Behavioral Sciences, 49, 282-292. 

APPENDIXES 



\section{Appendix 2.a}

\section{Square footage and sales price}

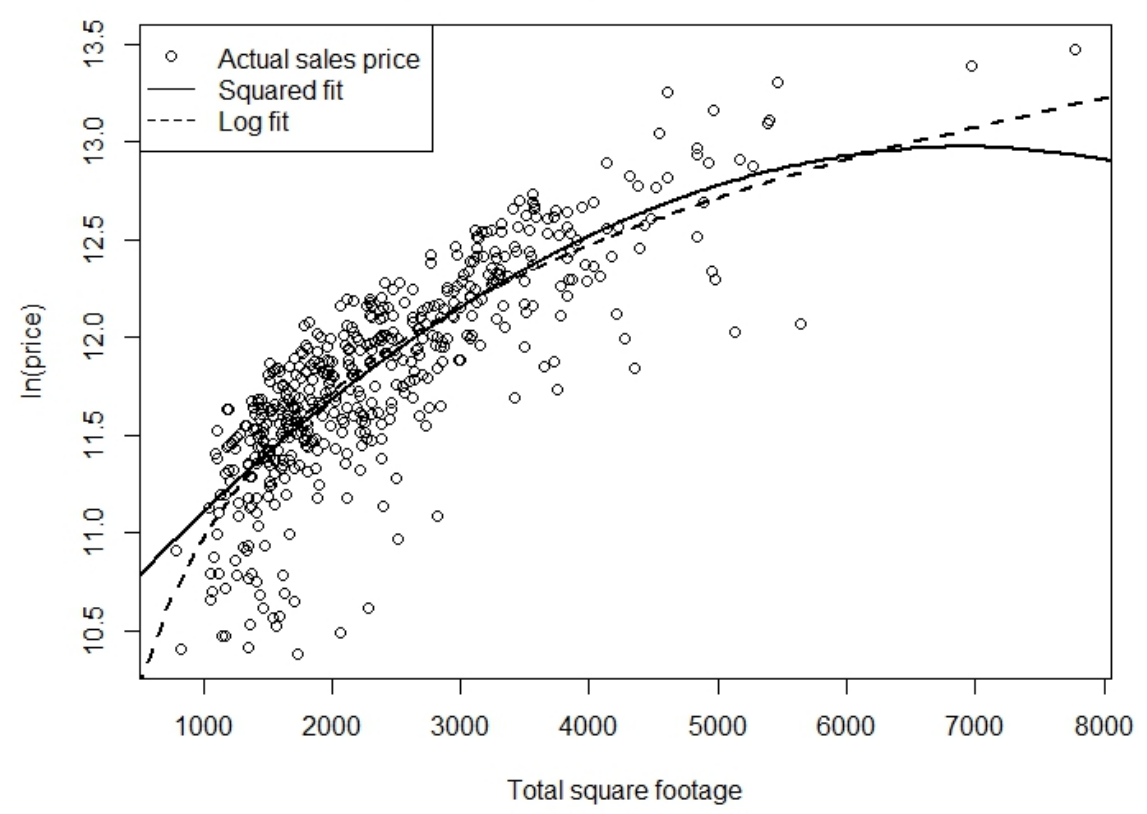

Figure AI. Relationship between house square footage and sales price, including the fitted regressions with squared and log functional forms.

\section{Appendix 2.b}

$$
\begin{aligned}
& R M S E=\sqrt{\frac{\sum_{i=1}^{n}\left(Y_{\text {pred }}-Y_{o b s}\right)^{2}}{n}} \\
& M A E=\frac{\sum_{i=1}^{n}\left|Y_{\text {pred }}-Y_{o b s}\right|}{n} \\
& S D E=\sqrt{\frac{\sum_{i=1}^{n}\left(Y_{\text {pred }}-Y_{o b s}\right)^{2}}{n-1}}
\end{aligned}
$$




\section{Appendix 3.a}

Table AI presents the experimental settings for each section of the results. In each section we use a different experimental approach, tackling one of two or both research objectives: (I) to quantitatively explore the market-level consequences of various behavioural models' assumptions regarding risky choices, (2) and the effect of the market-level bias that individual preferences for neighbourhood quality imply. We also include a reasoning behind these settings, in an attempt to elucidate what can be interpreted from the experiments.

Table AI. Experimental settings - columns 2 and 3 - for each section of the Results chapter. The fourth column indicates which research objective is tackled by each section and the fifth column gives the rationale for the experimental settings

\begin{tabular}{|c|c|c|c|c|}
\hline $\begin{array}{l}\text { Results } \\
\text { section }\end{array}$ & $\begin{array}{l}\text { Landscape } \\
\text { settings }\end{array}$ & $\begin{array}{l}\text { Gamma } \\
\text { settings }\end{array}$ & $\begin{array}{l}\text { Research } \\
\text { objective }\end{array}$ & Rationale \\
\hline 4.1 & Real & I & $I$ & $\begin{array}{l}\text { We quantify the market-level impact in a real } \\
\text { landscape, so that we can see which individual } \\
\text { risk behaviour model would best fit the } \\
\text { empirical data. }\end{array}$ \\
\hline 4.2 & Real & 0 & 2 & $\begin{array}{l}\text { By eliminating buyer's preferences for } \\
\text { neighbourhood quality, we can quantify the } \\
\text { specific bias in the revealed preference of flood } \\
\text { risk due to spatial correlation of risks and } \\
\text { amenities. }\end{array}$ \\
\hline 4.3 & Random & I & 1 and 2 & $\begin{array}{l}\text { Changing the landscape to random, we can } \\
\text { quantify the clean effect of individual risk } \\
\text { behaviour on an aggregate level. We can also } \\
\text { identify if there are any potential other sources } \\
\text { of multicollinearity apart from correlation } \\
\text { between risks and amenities that cause } \\
\text { systematic biases in market-level assessments } \\
\text { of risk. }\end{array}$ \\
\hline 4.4 & $\begin{array}{l}\text { Real and } \\
\text { Random }\end{array}$ & $0-2$ & 2 & $\begin{array}{l}\text { Across a range of gamma values we can } \\
\text { interpret (A) whether preference for } \\
\text { neighbourhood quality can indeed } \\
\text { systematically disturb macro-level risk } \\
\text { assessments in a real landscape, and (B) } \\
\text { whether randomising the landscape is a valid } \\
\text { way to control for multicollinearity in risks and } \\
\text { amenities. }\end{array}$ \\
\hline
\end{tabular}




\section{Appendix 3.b}

Below we present the dynamics of some model parameters changing over time as market evolves. Notably, the simulations converge to an equilibrium after some time. The number of time steps until an equilibrium is reached depends largely on gamma. Gamma represents buyers' sensitivity to neighbourhood quality in their selection of a property. On the sellers' side, the marginal implicit price of neighbourhood quality is captured by kriging. This addition in the price formation algorithm matches better with buyers' preferences when gamma is high, resulting in a faster convergence to an equilibrium. Meanwhile we see that the variance in residuals increases with gamma, as these residuals represent neighbourhood quality. To a lesser extent, the time to equilibrium depends also on the behavioural model of risk perception. The equilibrium value of flood risk is established faster when agents give a low weight to risk. A high risk perception results in a low number of sales of properties in the flood zone. While the capitalised price for flood risk is estimated through an analysis of these sales, the price estimate for flood risk can be highly volatile when sales are largely absent. We use the timelines in Fig. A2 to determine the limits of our analysis, as we are only interested in the equilibrium values. We visually analyse the time series of coefficients in the hedonic analysis, number of sales, residuals and mean sales prices to establish after how many time steps an equilibrium is reached. The equilibrium value for the flood zone coefficient in the hedonic analysis is generally reached within 30 time steps. Yet, some other parameters take longer to converge. Nonetheless, most of the simulations converge to an equilibrium within 40 time steps, which is 20 years of sales. Given that our simulations run for 50 time steps, we perform the rest of the analysis only for the equilibrium period and compare the values of the flood zone coefficient between $T=40$ and $T=50$, i.e. all sales over a period of five years. Model settings whereby convergence does not occur within 40 time steps are excluded from the analysis. 


\section{Appendixes}

Lot size coefficient over time

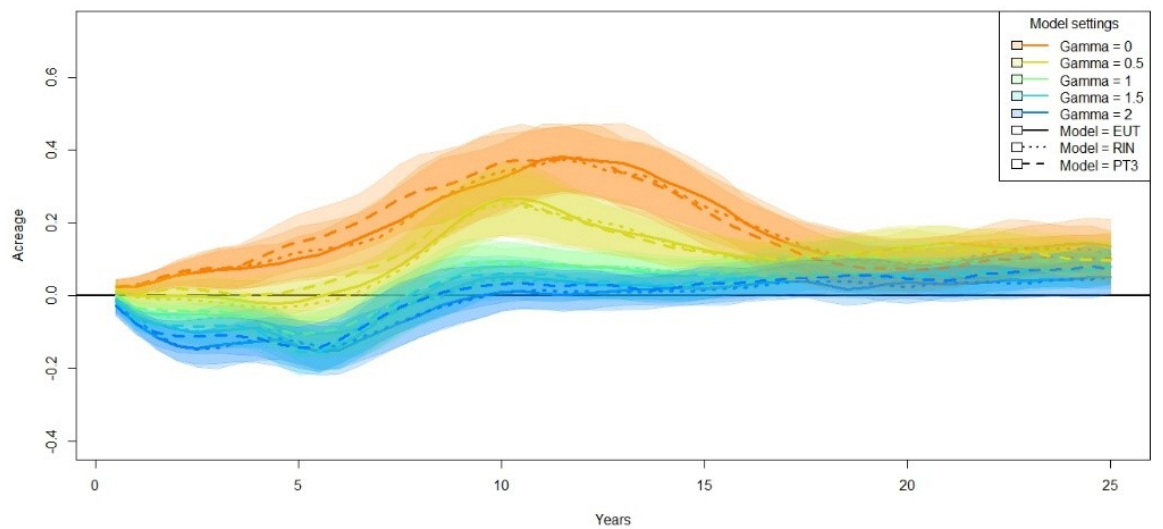

Age coefficient over time

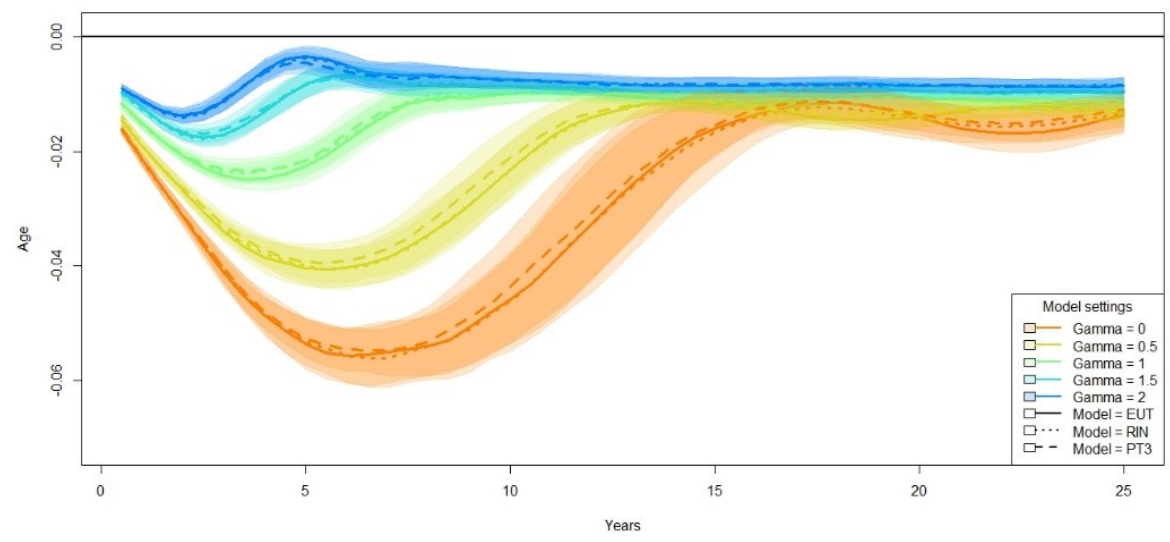


Appendixes

Number of bedrooms coefficient over time

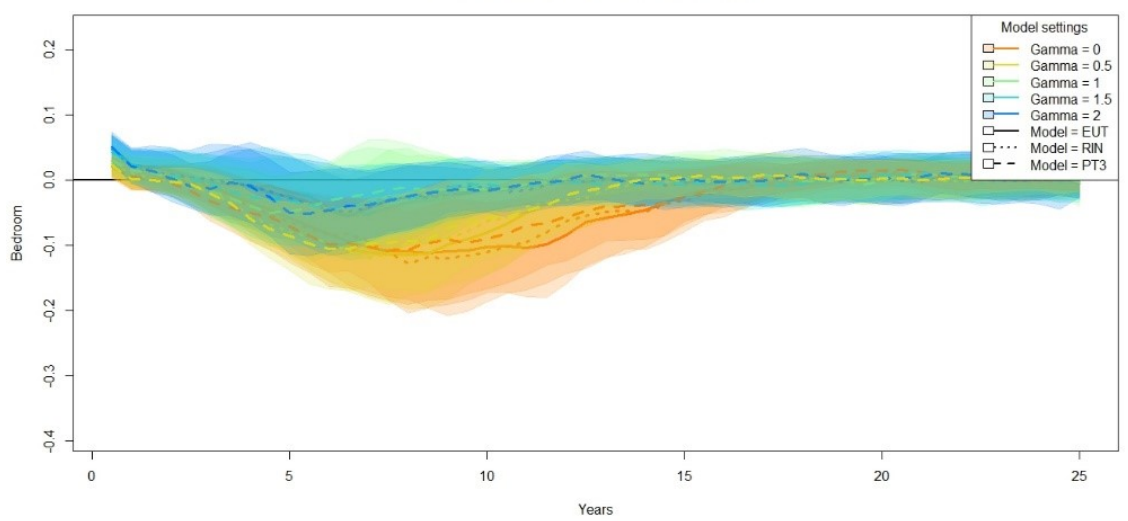

Flood discount over time

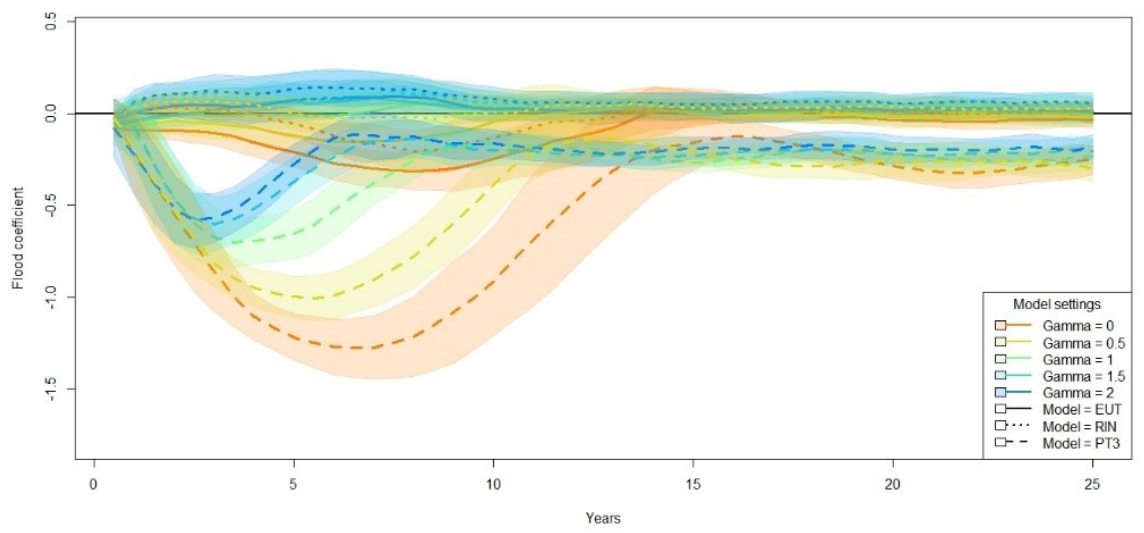

Mean sales price over time

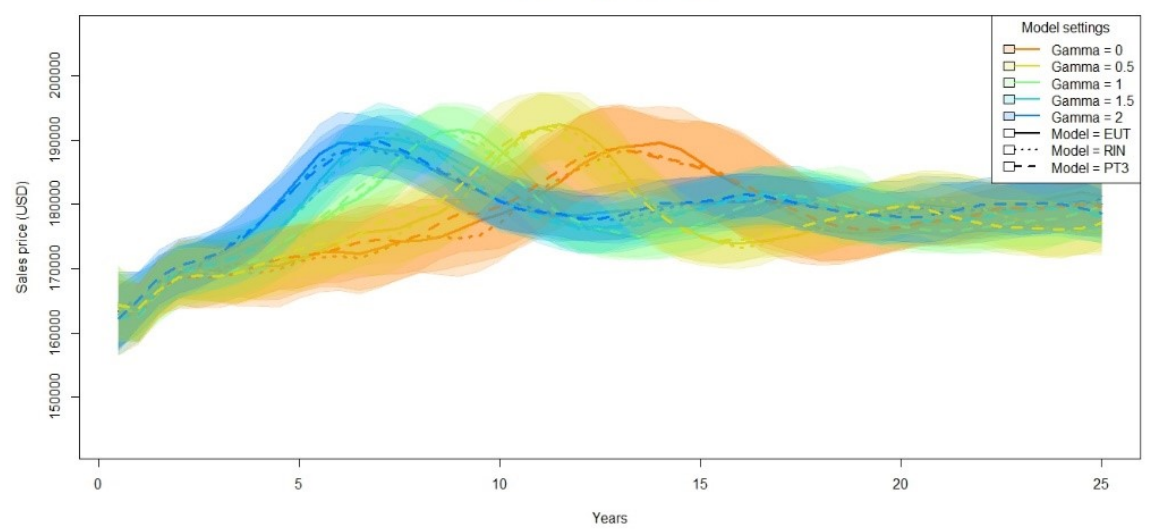




\section{Appendixes}

Flood properties sold over time

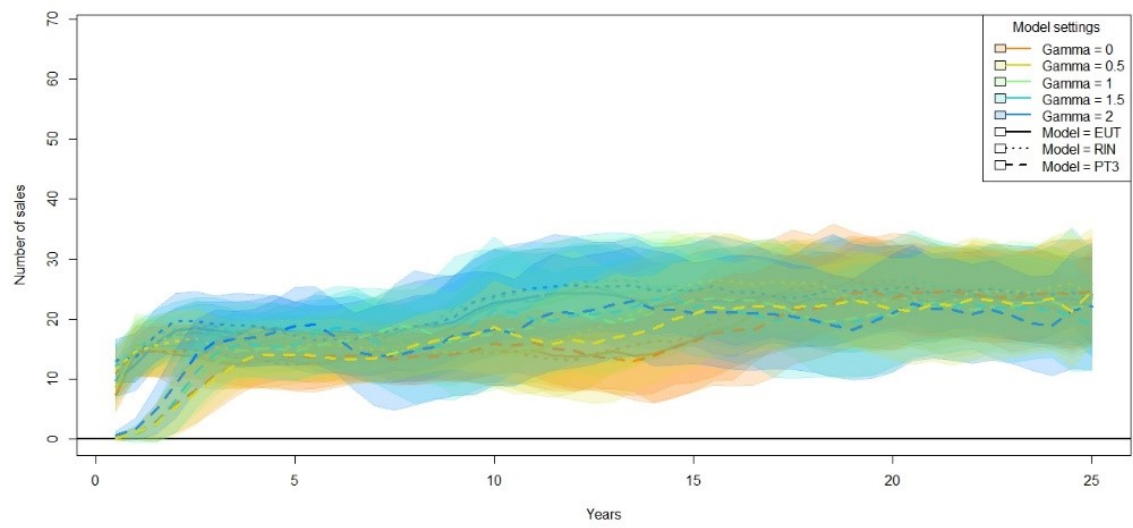

Standard deviation sales price over time

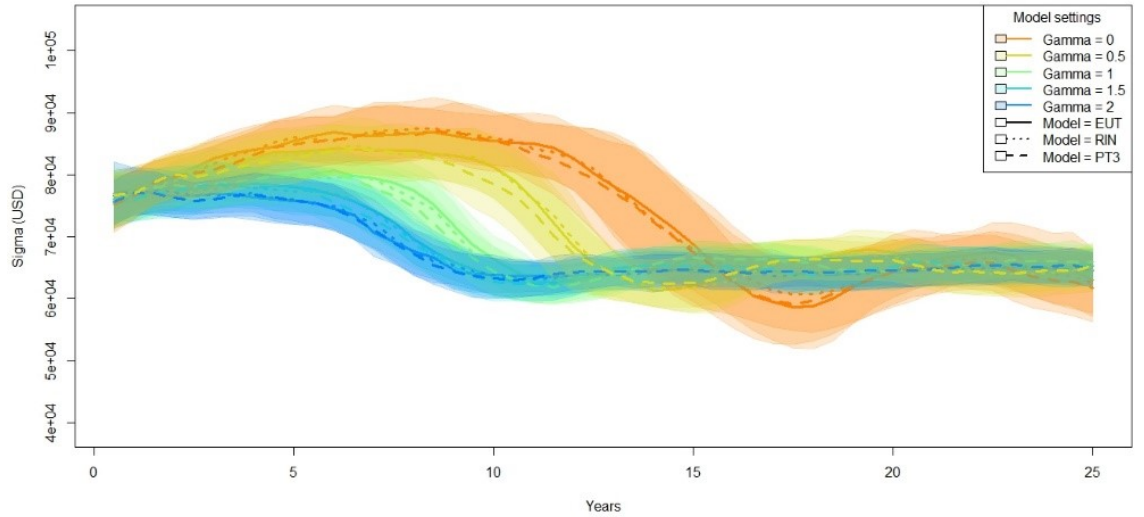

Standard deviation of residuals over time

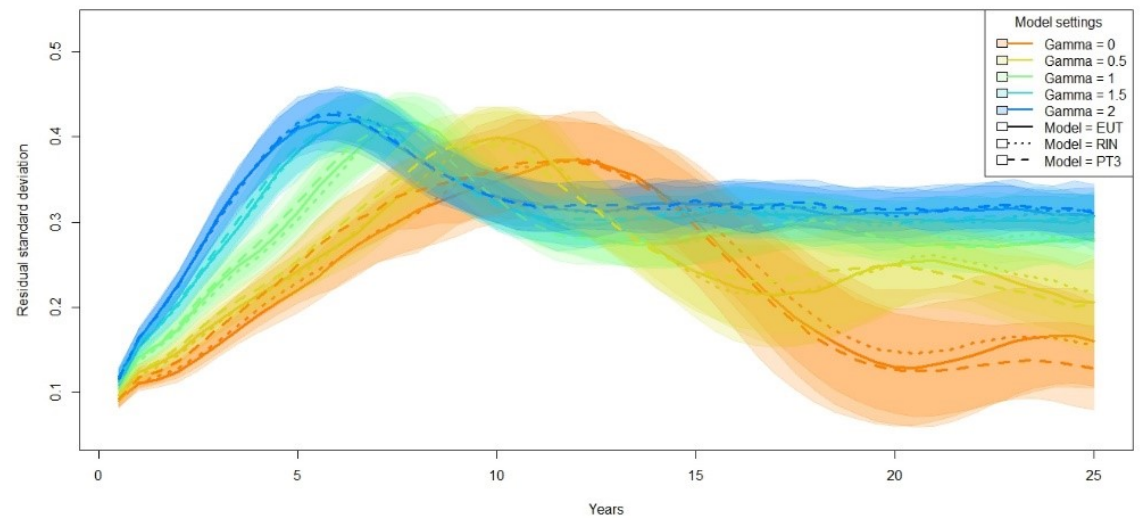




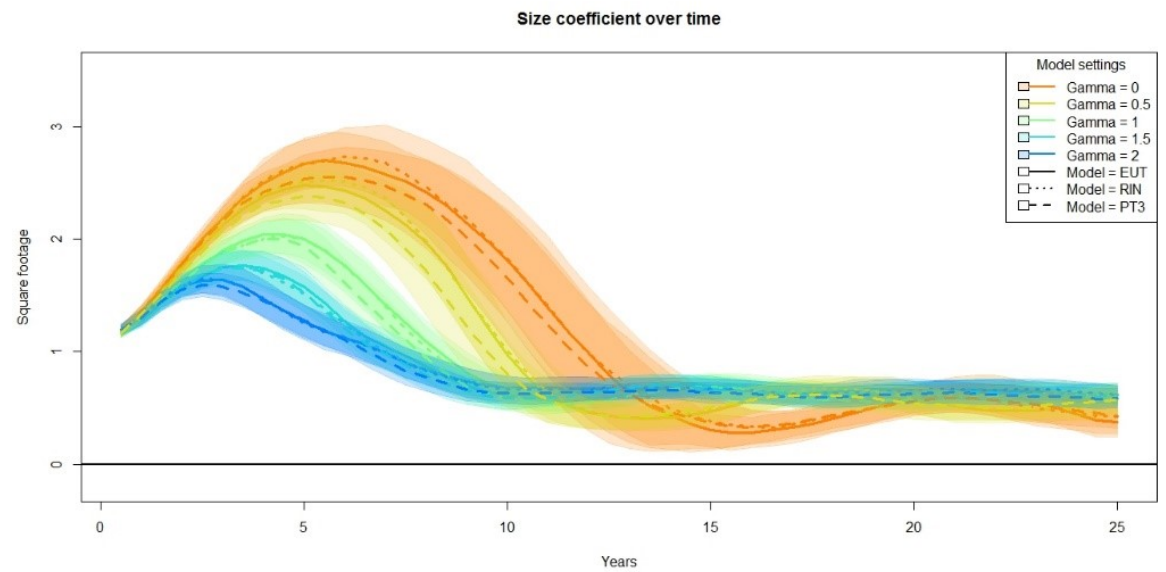

Figure A2. Model parameters converging to an equilibrium in the simulations. The time to reach an equilibrium depends largely on individual preferences for neighbourhood quality (the value of gamma), and somewhat on the behavioural risk perception model.

\section{Appendix 4.a}

Table A2. Summary Statistics of the Data $(N=348 I)$ for Beaufort, NC

\begin{tabular}{lllll}
\hline GIS parcel attribute & Average & St.dev. & Min & Max \\
\hline Number of bedrooms & 2.7 & 1.09 & 0 & 12 \\
House age & 40.2 & 29.9 & 0 & 274 \\
Sq. footage of a house & 1676 & 802.8 & 160 & 6080 \\
Lot size in acre & 1.03 & 3.09 & 0.005 & 42.39 \\
Whether a house is in I:I00 flood zone & 0.30 & 0.46 & 0 & $\mathrm{I}$ \\
Whether a house is in I:500 flood zone & 0.22 & 0.41 & 0 & $\mathrm{I}$ \\
\hline
\end{tabular}

\section{Appendix 4.b}

The following equations are meant to clarify how we calculated a percentage discount for properties in the flood zone driven by insurance costs. The same procedure applies to the risk attitude models. Eq. A4 shows the hedonic price of a property in the 100-year flood zone in the simulations without insurance (ins $=0$ ). The hedonic coefficient for the 100-year flood zone dummy is given by $a_{f z 100, i n s=0}$. The hedonic coefficients for all other property characteristics (i) are given by $a_{i}$.

$$
\ln \left(\text { price }_{f z 100, \text { ins }=0}\right)=\sum a_{i} X_{i}+C+a_{f z 100, \text { ins }=0} * 1
$$


Since we are using the natural log of sales prices in the hedonic analysis, the actual sales price is given by the e exponent of the hedonic coefficients and the property characteristics. Eq. A5 shows the price of a property located outside the flood zone.

price $_{\text {out }}=\exp \left(\sum a_{i} X_{i}+C\right)$

The price of a property located in the flood zone can be estimated by calculating the price as if it were outside the flood zone, and multiply it with the exponent of the coefficient of the 100-year flood zone dummy (Eq. A6).

price $_{f z 100, \text { ins }=0}=$ price $_{\text {out }} * \exp \left(a_{f z 100, \text { ins }=0}\right)$

The percentage difference between a property inside and outside the flood zone, all else being equal, is given by Eq. $A 7$.

$\Delta \operatorname{price}_{f z 100, \text { ins }=0}(\%)=\frac{\text { price }_{f \mathrm{z} 100, \text { ins }_{=0}-\text { price }_{\text {out }}}}{\text { price }_{\text {out }}} * 100$

Substituting $\frac{\text { rrice }_{f z 100, i n s=0}}{\text { price }_{\text {out }}}$ for $\exp \left(a_{f z 100, \text { ins }=0}\right)$ (Eq. A6 \& A7), we get Eq. A8, which shows how a percentage discount for a property located in the 100 -year flood zone is calculated directly from the coefficient of the 100 -year flood zone dummy in the hedonic analysis.

$\operatorname{sprice}_{f z 100, \text { ins }=0}(\%)=\left(\exp \left(a_{f z 100, \text { ins }=0}\right)-1\right) * 100$

In a similar way we can calculate the relative price discount for properties in the 100 year flood zone with and without insurance. We first compare the hedonic coefficients of the 100-year flood zone dummy of the simulations with insurance (ins=I) with those of the simulations without insurance (ins=0). The difference is given by $a_{f z 100, i n s}$ in Eq. A9.

$a_{f z 100, \text { ins }=1}=a_{f z 100, \text { ins }=0}+a_{f z 100, \text { ins }}$

Similar to Eq. A6 we can estimate the price for a property in the 100 -year zone with insurance by multiplying its price without insurance, all else being equal, with the $e$ exponent of $a_{f z 100, i n s}$. Thus, $a_{f z 100, i n s=0}$ can be substituted for $a_{f z 100, i n s}$ in Eq. A8 to calculate the percentage discount for a property in the 100 -year flood caused by insurance premiums. This can be calculated directly from the difference in the coefficients of the 100-year flood zone dummy $a_{f z 100, \text { ins }}$ between the simulations with and without insurance. The same equations are applied between the simulations with risk neglecting buyers and expected utility buyers to calculate a percentage discount for properties in the flood zone driven by changes in risk attitudes. 
$\operatorname{price}_{f z 100, \text { ins }=1}=\operatorname{price}_{f z 100, \text { ins }=0} * \exp \left(a_{f z 100, i n s}\right)$

The discount for properties in the 100-year flood zone in our simulations, $a_{f z 100}$, depends on the model of risk attitude $(R A)$ and insurance (ins). Both are either 0 or $\mathrm{I}$, and represent risk neglect or expected utility and false or true, respectively. We apply linear regression on the flood zone coefficients of the simulations $(N=200)$, with $R A$, ins and their interaction ins*RA as explanatory variables. Eq. All shows the variables that are predicted with this linear regression.

$a_{f z 100}($ ins,$R A)=I+a_{f z 100, i n s} *$ ins $+a_{f z 100, R A} * R A+a_{f z 100, i n s \times R A} *$ ins $*$ $R A$

(AII)

Namely, intercept $(I)$, discount for insurance $\left(a_{f z 100, i n s}\right)$, discount for flood risk $\left(a_{f z 100, R A}\right)$ and interaction effect of insurance and flood risk $\left(a_{f z 100, i n s \times R A}\right)$. These variables are used in Table 4.2. The values in Table 4.2 are constructed by substituting $a_{f z 100, i n s=0}$ in Eq. A8 for all of the variables $I, a_{f z 100, i n s}, a_{f z 100, R A}$ and $a_{f z 100, i n s \times R A}$ in Eq. AII. They represent row 2, columns 5, 2, 3 and 4 respectively.

\section{Appendix 5.a}

Table A3. Prior likelihood of hypothesised effect sizes for all statistical relations in the conceptual model, used to calculate the Bayesian posterior probabilities of the hypotheses

\begin{tabular}{|lllllll|}
\hline Variable & $\begin{array}{l}\text { Probability } \\
\text { density } \\
\text { function } \\
\text { priors }\end{array}$ & $\begin{array}{l}\text { Prior } \\
\text { expected } \\
\text { effect size }\end{array}$ & $\begin{array}{l}\text { Standard } \\
\text { deviation } \\
\text { effect size }\end{array}$ & $\begin{array}{l}\text { Minimum } \\
\text { effect size }\end{array}$ & $\begin{array}{l}\text { Maximum } \\
\text { effect size }\end{array}$ & Source \\
\hline $\begin{array}{l}\text { Sellers' decisions } \\
\text { Discount for } \\
\text { properties in } \\
\text { the I:I00 flood }\end{array}$ & Normal & 0.037 & 0.149 & $\mu-10 * \sigma$ & $\mu+10 * \sigma$ & Betran et al. \\
zone & & & & & $2018-$ Table 3: \\
\hline $\begin{array}{l}\text { Discount for } \\
\text { properties in } \\
\text { the I:500 flood } \\
\text { zone }\end{array}$ & Normal & -0.019 & 0.149 & $\mu-10 * \sigma$ & $\mu+10 * \sigma$ & year \\
\hline $\begin{array}{l}\text { Discount for } \\
\text { properties in } \\
\text { the I:I00 flood } \\
\text { zone after }\end{array}$ & Normal & -0.040 & 0.149 & $\mu-10 * \sigma$ & $\mu+10 * \sigma$ & Bet al. \\
\hline
\end{tabular}


Appendixes

\begin{tabular}{|c|c|c|c|c|c|c|}
\hline Variable & $\begin{array}{l}\text { Probability } \\
\text { density } \\
\text { function } \\
\text { priors }\end{array}$ & $\begin{array}{l}\text { Prior } \\
\text { expected } \\
\text { effect size }\end{array}$ & $\begin{array}{l}\text { Standard } \\
\text { deviation } \\
\text { effect size }\end{array}$ & $\begin{array}{l}\text { Minimum } \\
\text { effect size }\end{array}$ & $\begin{array}{l}\text { Maximum } \\
\text { effect size }\end{array}$ & Source \\
\hline \multicolumn{7}{|l|}{$\begin{array}{l}\text { experiencing a } \\
\text { flood }\end{array}$} \\
\hline $\begin{array}{l}\text { Discount for } \\
\text { properties in } \\
\text { the } 1: 500 \text { flood } \\
\text { zone after } \\
\text { experiencing a } \\
\text { flood }\end{array}$ & Normal & -0.055 & 0.149 & $\mu-10 * \sigma$ & $\mu+10 * \sigma$ & $\begin{array}{l}\text { Betran et al. } \\
2018 \text { - Table 3: } \\
\text { DID Inland } 500 \\
\text { year AF-BF }\end{array}$ \\
\hline $\begin{array}{l}\text { Discount for } \\
\text { properties that } \\
\text { were damaged } \\
\text { by the flood }\end{array}$ & Uniform & - & - & -1 & I & $\begin{array}{l}\text { Uninformative } \\
\text { (objective) } \\
\text { prior }\end{array}$ \\
\hline $\begin{array}{l}\text { Moving because } \\
\text { of flood risk }\end{array}$ & Binomial & - & - & 0 & I & $\begin{array}{l}\text { Uninformative } \\
\text { (objective) } \\
\text { prior }\end{array}$ \\
\hline $\begin{array}{l}\text { Moving out of } \\
\text { the flood zone }\end{array}$ & Binomial & - & - & 0 & I & $\begin{array}{l}\text { Uninformative } \\
\text { (objective) } \\
\text { prior }\end{array}$ \\
\hline $\begin{array}{l}\text { Perceived } \\
\text { likelihood of } \\
\text { damage (I = } \\
\text { highly unlikely, } \\
4 \text { = highly likely) }\end{array}$ & Normal & 1.46 & 0.79 & I & 4 & $\begin{array}{l}\text { Prior based on } \\
\text { the survey data }\end{array}$ \\
\hline $\begin{array}{l}\text { Fear towards } \\
\text { flooding }\end{array}$ & Normal & 2.75 & 1.00 & I & 4 & $\begin{array}{l}\text { Prior based on } \\
\text { the survey data }\end{array}$ \\
\hline Buyers' decisions & & & & & & \\
\hline $\begin{array}{l}\text { Willingness to } \\
\text { pay for } \\
\text { properties in } \\
\text { the } 1: 100 \text { flood } \\
\text { zone (compared } \\
\text { to outside) }\end{array}$ & Normal & 0.037 & 0.149 & $\mu-10 * \sigma$ & $\mu+10 * \sigma$ & $\begin{array}{l}\text { Betran et al. } \\
2018 \text { - Table 3: } \\
100 \text { year }\end{array}$ \\
\hline $\begin{array}{l}\text { Willingness to } \\
\text { pay for } \\
\text { properties in } \\
\text { the } 1: 500 \text { flood } \\
\text { zone (compared } \\
\text { to outside) }\end{array}$ & Normal & -0.019 & 0.149 & $\mu-10 * \sigma$ & $\mu+10 * \sigma$ & $\begin{array}{l}\text { Betran et al. } \\
2018 \text { - Table 3: } \\
500 \text { year }\end{array}$ \\
\hline $\begin{array}{l}\text { Willingness to } \\
\text { pay for } \\
\text { properties in } \\
\text { the I:I00 flood } \\
\text { zone (compared }\end{array}$ & Normal & -0.040 & 0.149 & $\mu-10 * \sigma$ & $\mu+10 * \sigma$ & $\begin{array}{l}\text { Betran et al. } \\
2018 \text { - Table 3: } \\
\text { DID Inland I00 } \\
\text { year AF-BF }\end{array}$ \\
\hline
\end{tabular}


Appendixes

\begin{tabular}{|c|c|c|c|c|c|c|}
\hline Variable & $\begin{array}{l}\text { Probability } \\
\text { density } \\
\text { function } \\
\text { priors }\end{array}$ & $\begin{array}{l}\text { Prior } \\
\text { expected } \\
\text { effect size }\end{array}$ & $\begin{array}{l}\text { Standard } \\
\text { deviation } \\
\text { effect size }\end{array}$ & $\begin{array}{l}\text { Minimum } \\
\text { effect size }\end{array}$ & $\begin{array}{l}\text { Maximum } \\
\text { effect size }\end{array}$ & Source \\
\hline $\begin{array}{l}\text { to outside) } \\
\text { after } \\
\text { experiencing a } \\
\text { flood }\end{array}$ & & & & & & \\
\hline $\begin{array}{l}\text { Willingness to } \\
\text { pay for } \\
\text { properties in } \\
\text { the } 1: 500 \text { flood } \\
\text { zone (compared } \\
\text { to outside) } \\
\text { after } \\
\text { experiencing a } \\
\text { flood }\end{array}$ & Normal & -0.055 & 0.149 & $\mu-10 * \sigma$ & $\mu+10 * \sigma$ & $\begin{array}{l}\text { Betran et al. } \\
2018 \text { - Table 3: } \\
\text { DID Inland } 500 \\
\text { year AF-BF }\end{array}$ \\
\hline $\begin{array}{l}\text { Avoid buying } \\
\text { property in the } \\
\text { flood zone (I = } \\
\text { strongly } \\
\text { disagree, } 4= \\
\text { strongly agree) }\end{array}$ & Uniform & - & - & I & 4 & $\begin{array}{l}\text { Uninformative } \\
\text { (objective) } \\
\text { prior }\end{array}$ \\
\hline $\begin{array}{l}\text { Perceived } \\
\text { likelihood of } \\
\text { damage ( } 1= \\
\text { highly unlikely, } \\
4=\text { highly likely) }\end{array}$ & Normal & 1.65 & 0.85 & I & 4 & $\begin{array}{l}\text { Prior based on } \\
\text { the survey data }\end{array}$ \\
\hline $\begin{array}{l}\text { Fear towards } \\
\text { flooding }\end{array}$ & Normal & 2.75 & 1.00 & I & 4 & $\begin{array}{l}\text { Prior based on } \\
\text { the survey data }\end{array}$ \\
\hline $\begin{array}{l}\text { Take flood risk } \\
\text { into account in } \\
\text { buying a new } \\
\text { property }\end{array}$ & Binomial & - & - & 0 & 1 & $\begin{array}{l}\text { Uninformative } \\
\text { (objective) } \\
\text { prior }\end{array}$ \\
\hline $\begin{array}{l}\text { Actively looking } \\
\text { for property } \\
\text { outside the } \\
\text { flood zone }\end{array}$ & Binomial & - & - & 0 & I & $\begin{array}{l}\text { Uninformative } \\
\text { (objective) } \\
\text { prior }\end{array}$ \\
\hline $\begin{array}{l}\text { Willingness to } \\
\text { pay for } \\
\text { properties that } \\
\text { require flood } \\
\text { insurance } \\
\text { (compared to } \\
\text { properties that } \\
\text { don't) }\end{array}$ & Uniform & - & - & -1 & 1 & $\begin{array}{l}\text { Uninformative } \\
\text { (objective) } \\
\text { prior }\end{array}$ \\
\hline
\end{tabular}




\begin{tabular}{|c|c|c|c|c|c|c|}
\hline Variable & $\begin{array}{l}\text { Probability } \\
\text { density } \\
\text { function } \\
\text { priors }\end{array}$ & $\begin{array}{l}\text { Prior } \\
\text { expected } \\
\text { effect size }\end{array}$ & $\begin{array}{l}\text { Standard } \\
\text { deviation } \\
\text { effect size }\end{array}$ & $\begin{array}{l}\text { Minimum } \\
\text { effect size }\end{array}$ & $\begin{array}{l}\text { Maximum } \\
\text { effect size }\end{array}$ & Source \\
\hline $\begin{array}{l}\text { Adopt } \\
\text { protective } \\
\text { measures } \\
\text { against flood } \\
\text { damage in new } \\
\text { home }\end{array}$ & Binomial & - & - & 0 & I & $\begin{array}{l}\text { Uninformative } \\
\text { (objective) } \\
\text { prior }\end{array}$ \\
\hline $\begin{array}{l}\text { Get flood } \\
\text { insurance for } \\
\text { new home }\end{array}$ & Binomial & - & - & 0 & I & $\begin{array}{l}\text { Uninformative } \\
\text { (objective) } \\
\text { prior }\end{array}$ \\
\hline
\end{tabular}

\section{Appendix 5.b - Buyer survey}

Thank you for taking the time to help with our research on flood risk. The information you provide will be used to better understand market forces in housing. The questions should take about I0-15 minutes of your time.

The questions are divided into five sections:

- Introduction [qualifying questions]

- Flood Risk Perceptions

- Protection from Flood Risk

- Your Last Home Purchase

- Experience with Flooding

- Demographics

\section{Introduction [qualifying questions]}

I. In what state do you live?

a. [dropdown menu of states]

2. Do you own your own home?
a. Yes
b. No

3. Did you purchase the home you live in within the past $\mathbf{I} \mathbf{2}$ months?
a. Yes [proceed to Q6]
b. No [proceed to Q5]

4. Are you currently in the market to purchase an owner-occupied house?
a. Yes
b. No [disqualify and exit survey] 
5. Have you identified a property on which you are considering making or have made an offer?

a. Yes [proceed to Q7]

b. No [disqualify and exit survey]

6. [Screener for current buyers] What interest rate do you expect to pay on your home mortgage?
a. $6-7 \%$ [disqualify and exit survey]
b. $7-8 \%$ [disqualify and exit survey]
c. $8-9 \%$ [disqualify and exit survey]
d. $9-10 \%$ [disqualify and exit survey]
e. $\quad 10-11 \%$ [disqualify and exit survey]
f. $>11 \%$ [disqualify and exit survey]
g. None of these [proceed with part II of the survey]

7. [Screener for recent buyers] What interest rate are you currently paying on your mortgage?
a. $7-8 \%$ [disqualify and exit survey]
b. $8-9 \%$ [disqualify and exit survey]
c. $9-10 \%$ [disqualify and exit survey]
d. $10-11 \%$ [disqualify and exit survey]
e. $>11 \%$ [disqualify and exit survey]
f. None of these [proceed with part II of the survey]
g. I do not have a mortgage on the house I bought [proceed with part II of the survey]

\section{Perceptions about Flood Risk}

DEFINITIONS: The Federal Emergency Management Agency (FEMA) identifies flood hazards through statistical information such as data for river flow, storm tides, hydrologic/hydraulic analyses and rainfall and topographic surveys. They outline flood risk areas with certain probabilities of flooding, the 100 -year flood zone and 500-year flood zone. They are defined as follows:

500-year flood zone - The area that has a .2 percent chance of being inundated by a flood event in any given year.

100-year flood zone - The area that has a I percent chance of being inundated by a flood event in any given year. 
I. [for recent buyers] How likely is it that your house will get damage from flooding?

[for current buyers] How likely is it that the house that you would like to buy will get damage from flooding?

[4-point scale with only end labels: very likely, very unlikely]

2. Please indicate your agreement or disagreement with each of the following statements relating to flood risk using the scale provided.

[4-point scale with only end labels: strongly agree, strongly disagree]

a. If a property is in a 100-year flood zone, I would never buy this property

b. If a property is in a 500-year flood zone, I would never buy this property

c. If a property has been flooded before, I would not never buy this property

d. I'm ok with living in a flood zone

e. The risk of flooding deters me from buying property in the (100/500-year) flood zone.

f. The cost of flood insurance deters me from buying property in the 100-year flood zone.

g. I worry about the risk of flooding

h. I fear the consequences of flooding

Space for comments on Perceptions of Flood Risk

III. Protecting yourself from Flood Risk

I. Which of the following have you done or are you planning to do as ways to minimize your personal damage in case of flooding? (Select all that apply)

a. Installation of flood openings (vents), routes for water to drain

b. Get flood insurance for your home

c. Selling the house and move to a location outside the flood zone

d. Elevating your home/Lifting the property or building the house on pilings

e. Abandon lowest floor

f. None of these

2. Please rate how effective you think each of the following measures is for reducing your risk?

[5-point scale with end labels: very effective/very ineffective + Not sure]

a. Installation of flood openings (vents), routes for water to drain 
b. Get flood insurance for your home

c. Selling the house and move to a location outside the flood zone

d. Elevating your home/Lifting the property or building the house on pilings

e. Abandon lowest floor

3. Please rate how expensive you think each of the following measures is?

[5-point scale with end labels: very expensive/very inexpensive + Not sure]

a. Installation of flood openings (vents), routes for water to drain

b. Get flood insurance for your home

c. Selling the house and move to a location outside the flood zone

d. Elevating your home/Lifting the property or building the house on pilings

e. Abandon lowest floor

4. How easy or difficult do you consider these measures be for the home you have bought? [5-point scale with end labels: very easy/very difficult + Not sure]

a. Installation of flood openings (vents), routes for water to drain

b. Get flood insurance for your home

c. Selling the house and move to a location outside the flood zone

d. Elevating your home/Lifting the property or building the house on pilings

e. Abandon lowest floor

\section{Your Home Purchase}

[for recent buyers] The questions in this section relate to your purchase of the house you now live in. We ask you to remember the process you went through when you bought your house.

[for current buyers] The questions in this section relate to the house you would like to purchase. We ask you to answer these questions about the process you go through related to your purchase.

I. [for recent buyers] How much was the house listed for when you offered the first bid on your house?

[for current buyers] How much is the house currently listed for?

a. US dollars 
b. Don't know

2. [for recent buyers] How much did you pay for your house? [for current buyers] How much do you hope to pay for your house?

a. US dollars

3. [for recent buyers] Do you currently have a mortgage on the house you purchased?

[for current buyers] Are you planning to have a mortgage on your new home?
a. Yes
b. No [proceed to Q4]

4. [for recent buyers] What percentage of your purchase price is financed with a commercial broker or bank?

[for current buyers] What percentage of your purchase price are you planning to finance with a commercial broker or bank?
a. $76 \%$ or more
b. $51-75 \%$
c. $26 \%-50 \%$
d. $1-25 \%$
e. Uncertain/Don't remember

5. Thinking back to when you were looking for a new home, what were your "must have" criteria for purchase?
a. No. of bedrooms (please specify)
b. A minimum square footage (please specify)
c. A minimum number of bathrooms (please specify)
d. Within a certain school district
e. A garage
f. Maximum years old (please specify)
g. Minimum years old (please specify)
h. Acres of land (please specify)
i. Live on the water front
j. Live near the water
k. Located in certain neighborhood (please specify)
I. House had to be outside the 500-year flood zone
m. House had to be outside the 100-year flood zone
n. Proximity to work (please specify) minutes
o. Other (please specify)
p. Don't remember

5 b. In what price range are/were your looking for a house? 

q. Upper limit US dollars
r. Lower limit US dollars

6. [for recent buyers] Where did you live prior to purchasing your current home?

[for current buyers] Where do you currently live?
a. The same city/area where I live now
b. Another city/area in the same state
c. In another state (please specify)
d. Abroad (i.e. out of the country)

7. [for recent buyers] Please provide the street address and city of your home.

[for current buyers] Please provide the street address and city of the house that you would like to buy.
a. Street address:
b. City:
c. State:

8. [for recent buyers] Is your home in one of the flood zones?

[for current buyers] Is the house you would like to buy in one of the flood zones?
a. Yes, it is in the 500-year flood plain
b. Yes, it is in the 100-year flood plain
c. Yes, but it's in a different flood zone, namely
d. No, the house is outside of a flood zone [skip QI0 and Q14]
e. I don't know [skip QI0 and Q I4]

9. Thinking back to when you were looking for a new home, did you think about flood zones at all?
a. Yes
b. No

10. [for recent buyers] Did the risk of flooding affect the price you paid for the house?

[for current buyers] Does the risk of flooding affect the price you are willing to pay for the house?
a. Yes
b. No [proceed to Q12]
c. Uncertain [proceed to QI2]

I I. [if yes] How much would the house be worth if it was not at risk of flooding?

a. US dollars 
b. Uncertain

12. [for recent buyers] Do you carry flood insurance on your home? [for current buyers] Do you plan to get flood insurance on your new home?
a. Yes
b. No [proceed to QI5]
c. Uncertain [proceed to Q15]

13. [for recent buyers] How much are you paying for flood insurance? [for current buyers] How much do you expect to pay for flood insurance for your new home?
a.
b. Uncertain USD per month

14. [for recent buyers] Did this flood insurance premium affect the amount you paid for the property?

[for current buyers] Does this flood insurance premium affect the amount you (would) offer for the property?
a. Yes
b. No
c. Uncertain

I5. [for recent buyers] If you were to experience a flood where you now live, what would you expect your personal damage to be and what percent of that total would be covered by insurance? [for current buyers] If there would be a flood where you are planning to live, what would you expect your personal damage to be and what percent of that total would be covered by insurance?

a. Total estimated cost of damages in US dollars

b. Percent of above total covered by insurance

Space for comments on your last home purchase

\section{Experience with Flooding}

I. Did you ever personally experience a major flooding event in your town?
a. Yes
b. No [continue with $\mathrm{VI}$ - demographics]
c. Don't remember

2. Where did your live at the time of the flood? 

a. City
b. State

3. When did this flood event take place?
a. Month
b. Year

4. Did you experience any damages caused by this flooding event?
a. Yes
b. No [continue with $\mathrm{VI}$ - demographics]
c. Don't remember [continue with $\mathrm{VI}$ - demographics]

5. How much damage approximately and what percent of that total was covered by insurance?

a. Total estimated cost of damages

b. Percent of above total covered by insurance

\section{Space for comments on your experience with flooding}

\section{Demographics}

I. How do you learn about flood risk? (Select all that apply)

a. I do not go out of my way to learn about flood risk

b. Personal experience with flooding

c. Experience of family/friends/colleagues

d. Experience of neighbors

e. FEMA flood maps

f. News media

g. Other sources, Please specify

2. [For each answer in QI] Please indicate why:
a. I trust this source of information
b. My situation is similar to the source's situation
c. I have no alternative information
d. It is the best available information source
e. Other reasons, Please specify

3. Approximately, what is your total annual household income?
a. Less than $\$ 30,000$
b. $\$ 30,000$ - $\$ 49,999$
c. $\$ 50,000-\$ 74,999$
d. $\$ 75,000$ - $\$ 99,999$
e. $\$ 100,000$ - $\$ 149,999$
f. $\$ 150,000-\$ 199,999$
g. $\$ 200,000$ or more
h. Prefer not to answer 
4. Would you like to receive an online summary of the results of this study?
a. Yes
b. No

5. For follow-up purposes only please provide the following information.

First name

Last name

Company

Email address

Phone number (or retype email address)

Room for final comments/suggestions/requests

Thank you for taking part in this important study.

\section{Appendix 5.c - Seller survey}

Thank you for taking the time to help with our research on flood risk. The information you provide will be used to better understand market forces in housing. The questions should take about I0-15 minutes of your time.

The questions are divided into five sections:

- Introduction [Qualifying questions]

- Perceiving Flood Risk

- Protecting yourself from flood risk

- Selling your Home

- Experience with Flooding

- Demographics

\section{Introduction [Qualifiers]}

I. In what state do you live?

a. [dropdown menu of states]

2. Do you own your own home?
a. Yes 
b. No [Disqualify and exit]

3. Are you currently selling your house or have you sold your house within the last twelve months?

a. My house is currently on the market

b. I've sold my house within the last twelve months

c. Neither [Disqualify and exit]

4. [If "sold"] Where (in which state) was the house you recently sold?

a. State: [Disqualify and exit if not a flood risk state]

b. Outside the US [Disqualify and exit]

5. [Screener] What was the interest rate for your mortgage when you sold your house?
a. $7-8 \%$ [Disqualify and exit]
b. $8-9 \%$ [Disqualify and exit]
c. $9-10 \%$ [Disqualify and exit]
d. $10-11 \%$ [Disqualify and exit]
e. $>11 \%$ [Disqualify and exit]
f. None of these [Proceed with survey]

\section{Perceptions of Flood Risk}

DEFINITIONS: The Federal Emergency Management Agency (FEMA) identifies flood hazards through statistical information such as data for river flow, storm tides, hydrologic/hydraulic analyses and rainfall and topographic surveys. They outline flood risk areas with certain probabilities of flooding, the 100-year flood zone and 500-year flood zone. They are defined as follows:

500-year flood zone - The area that has a .2 percent chance of being inundated by a flood event in any given year.

100-year flood zone - The area that has a I percent chance of being inundated by a flood event in any given year.

I. [If selling] How likely is it that your house will get damage from flooding?

[If sold] How likely is it that the house that you have sold will get damage from flooding?

[4-point scale with only end labels: very likely, very unlikely]

2. Please indicate your agreement or disagreement with each of the following statements relating to flood risk using the scale 
provided. [4-point scale with only end labels: strongly agree, strongly disagree]

a. If a property is in a 100-year flood zone, I would never buy this property

b. If a property is in a 500-year flood zone, I would never buy this property

c. If a property has been flooded before, I would not never buy this property

d. I'm ok with living in a flood zone

e. The risk of flooding deters me from buying property in the (100/500-year) flood zone.

f. The cost of flood insurance deters me from buying property in the 100-year flood zone.

g. I worry about the risk of flooding

h. I fear the consequences of flooding

Space for comments on perceptions of flood risk

\section{Protecting yourself from Flood Risk}

I. [If selling] Which of the following did you do or are still planning to do for the home you are selling as ways to minimize your personal damage in case of flooding?

[If sold] Which of the following did you do for the home you sold as ways to minimize your personal damage in case of flooding? (Select all that apply)

c. Installation of flood openings (vents), routes for water to drain

d. Get flood insurance for your home

e. Selling the house and move to a location outside the flood zone

f. Elevating your home/Lifting the property or building the house on pilings

g. Abandon lowest floor

h. None of these

2. Please rate how effective you think each of the following measures is for reducing your risk?

[5-point scale with end labels: very effective/very ineffective + Not sure]

a. Installation of flood openings (vents), routes for water to drain

b. Get flood insurance for your home 
c. Selling the house and move to a location outside the flood zone

d. Elevating your home/Lifting the property or building the house on pilings

e. Abandon lowest floor

3. Please rate how expensive you think each of the following measures is?

[5-point scale with end labels: very expensive/very inexpensive + Not sure]

a. Installation of flood openings (vents), routes for water to drain

b. Get flood insurance for your home

c. Selling the house and move to a location outside the flood zone

d. Elevating your home/Lifting the property or building the house on pilings

e. Abandon lowest floor

4. [If selling] How easy or difficult do you consider these measures be for the home you are selling?

[If sold] How easy or difficult do you consider these measures be for the home you have sold?

[5-point scale with end labels: very easy/very difficult + Not sure]

a. Installation of flood openings (vents), routes for water to drain

b. Get flood insurance for your home

c. Selling the house and move to a location outside the flood zone

d. Elevating your home/Lifting the property or building the house on pilings

e. Abandon lowest floor

\section{Selling Your Home}

The questions in this section relate to you selling your home. Several questions ask you to remember the process you went through when you sold your house or what you are going through as you sell your house.

I. [If selling] What is the main reason you are selling your house? (Select no more than three)

[If sold] What was the main reason you sold your house? (Select no more than three)

a. Wanted a bigger, newer or nicer house 
b. To be closer to work/Job change

c. Could not afford property anymore

d. Retirement/downsizing

e. Medical reasons

f. Related to a divorce or separation

g. Dissatisfaction with neighborhood

h. Risk of natural hazards in that area

i. Neighborhood deterioration

j. Other (please specify)

2. What was your initial list price when you first put your property on the market?

a. US dollars

3. [If selling] What is your current list price?

[If sold] For how much did you sell your house?

a. Sales Price: US dollars

b. Current List Price: US dollars

4. [If selling] What is the lowest price you would accept for your house?

[If sold] What was the lowest price you would have accepted for your house?

a. US dollars

b. Uncertain

5. [If selling] How long has your house been for sale?

[If sold] How long did it take to sell your house?

a. Less than 3 months

b. 3-6 months

c. 7-12 months

d. I-2 years

e. 3 years or more

f. Uncertain/Can't remember

6. [If selling] Approximately, how many showings of your house have you had?

[If sold] Approximately, how many showings of your house did you have?
a. None
b. I-3
c. 4-10
d. II-20
e. More than 20
f. Uncertain 
7. [If selling] Which of the following statements best represents your feelings about selling your house? (Select all that apply)
a. My property is likely to sell quickly
b. I will get a good price for my property.
c. I am generally pessimistic about the chances of selling my property.
d. I will likely end up taking less than my list price for my property.
e. Uncertain

8. [If sold] Which of the following statements best represents your feelings about selling your house? (Select all that apply)
a. My property sold quickly.
b. I got a good price for my property.
c. It took a long time to sell my property.
d. I reduced my list price in order to sell my property.
e. Uncertain

9. [If selling] Please provide the following information for your house:

[If sold] Please provide the following information for the house you sold:
a. No. of bedrooms (please specify)
b. Square footage (please specify*)
c. No. of bathrooms (please specify)
d. A garage $(\mathrm{Y}, \mathrm{N})$
e. Built in year (please specify*)
f. Acres of land (please specify*)
g. Live on the water front $(\mathrm{Y}, \mathrm{N})$
h. Live near the water $(\mathrm{Y}, \mathrm{N})$
i. Outside the flood zone $(\mathrm{Y}, \mathrm{N})$
*a rough estimation is fine

I0. [If selling] Where are you planning to move?
a. The same city/area where I live now
b. Another city/area in the same state
c. In another state (please specify)
d. Abroad (i.e. out of the country)

[If sold] Where did you move after selling your home?
e. The same city/area where I used to live
f. Another city/area in the same state
g. In another state (please specify)
h. Abroad (i.e. out of the country) 
II. [If selling] Please provide the street address and city of your home.

[If sold] Please provide the street address and city of the house which you sold.

a. Street address:

b. City:

12. [If selling] Is your home in one of the flood zones?

[If sold] Is the home you sold in one of the flood zones?
a. Yes, it is in the 100-year flood zone
b. Yes, it is in the 500-year flood zone
c. Yes, but it's in a different flood zone, namely
d. No, our house is outside of a flood zone [proceed to QI4]
e. I don't know [proceed to Q14]

13. [If in flood zone] Approximately how much do you think your property would be worth if it was located outside the flood zone?

a. Please specify amount US dollars

b. Uncertain

14. [If selling] Does the house you are selling have any visible damage from flooding events?

[If sold] Did the house you sold have any visible damage from flooding events?
a. Yes
b. No [proceed to Q17]
c. Don't know [proceed to Q17]

15. [If selling] Do these damages affect the value of the property in any way?

[If sold] Did these damages affect the value of the property in any way?
a. Yes
b. No [proceed to Q17]
c. Don't know [proceed to QI7]

16. How much would your property be worth if your property wasn't damaged?

a. Please specify amount US dollars

17. [If selling] Is your neighborhood visibly affected by damage from flooding events?

[If sold] Is the neighborhood of the house you sold visibly affected by damage from flooding events? 

a. Yes
b. No [proceed to Q20]
c. Don't know [proceed to Q20]

I 8. [If selling] Do these damages affect the value of your property in any way?

[If sold] Did these damages affect the value of your property in any way?
a. Yes
b. No [proceed to Q20]
c. Don't know [proceed to Q20]

19. How much would your property be worth if the neighborhood wasn't affected by floods?

a. Please specify amount US dollars

20. [If selling] Do you carry flood insurance on your home? [If sold] Did you carry flood insurance on the home you sold?
a. Yes
b. No [proceed to IV - Experience with flooding]
c. Uncertain [proceed to IV - Experience with flooding]

2I. [If selling] What do you pay monthly for flood insurance? [If sold] What did you pay monthly for flood insurance?

\section{a. \\ b. Uncertain \\ Space for Comments on Selling Your Home}

\section{Experience with Flooding}

I. Did you ever personally experience a major flooding event in your town?
a. Yes
b. No [proceed to Q7]
c. Don't remember [proceed to Q7]

2. Where did your live at the time of the flood?
a. City
b. State

3. When did this flood event take place?
a. Month
b. Year 
4. Did your house sustain damage from the flood you mention above?
a. Yes
b. No [proceed to Q7]

5. What was the total damage to your house and property and what percent of that total was covered by insurance?
a. Total damage: US dollars
b. Percent covered by flood insurance:

6. [If selling] If you were to experience a flood in your home, what would you expect your personal damage to be and what percent of that total would be covered by insurance? [If sold] If you had experienced a flood at the house you just sold, what would you have expected your personal damage to be and what percent of that total would have been covered by insurance?

a. Total estimated cost of damages US dollars

b. Percent of above total covered by insurance

Space for comments on your experience with flooding

\section{Demographics}

I. How do you learn about flood risk? (Select all that apply)
a. I do not go out of my way to learn about flood risk
b. Personal experience with flooding
c. Experience of family/friends
d. Experience of neighbors
e. FEMA flood maps
f. News media
g. Other
sources,
Please

specify

2. [For each answer in QI] Please indicate why: (Select all that apply)
a. I trust this source of information
b. My situation is similar to the source's situation
c. I have no alternative information
d. It is the best available information source
e. Other
reasons,
Please

specify

3. Approximately, what is your total annual household income?
a. Less than $\$ 30,000$
b. $\$ 30,000-\$ 49,999$ 

c. $\$ 50,000-\$ 74,999$
d. $\$ 75,000-\$ 99,999$
e. $\$ 100,000-\$ 149,999$
f. $\$ 150,000-\$ 199,999$
g. $\$ 200,000$ or more
h. Prefer not to answer

4. Would you like to receive an online summary of the results of this study?
a. Yes
b. No

5. For follow-up purposes only please provide the following information.

First name

Last name

Company

Email address

Phone number (or retype email address)

Room for final comments/suggestions/requests

Thank you for taking part in this important study. 



\section{SUPPLEMENTARY}

\section{List of Abbreviations}

$\begin{array}{ll}\text { ABM } & \text { Agent-based modelling } \\ \text { ABMs } & \text { Agent-based models } \\ \text { (an) ABM } & \text { (an) Agent-based model } \\ \text { ACE } & \text { Agent-Based Computational Economics } \\ \text { AVONA } & \text { Analysis of variance } \\ \text { CBA } & \text { Cost-Benefit Analysis } \\ \text { CHANS } & \text { Coupled Human And Natural Systems } \\ \text { CHES } & \text { Coupled Human-Environment Systems } \\ \text { CNH } & \text { Coupled Natural and Human } \\ \text { Eq. } & \text { Equation } \\ \text { EU } & \text { Expected Utility } \\ \text { EUT } & \text { Expected Utility Theory } \\ \text { FEMA } & \text { Federal Emergency Management Agency } \\ \text { Fig. } & \text { Figure } \\ \text { FL } & \text { Florida } \\ \text { GIS } & \text { Geographic Information System } \\ \text { HA } & \text { Hedonic Analysis } \\ \text { HA-ABM } & \text { Hedonic Analysis integrated with Agent-Based Modelling } \\ \text { HA-ACE } & \text { Hedonic Analysis integrated with Agent-Based Computational } \\ \text { MAE } & \text { Economics } \\ \text { NC } & \text { Mean Absolute prediction Error } \\ \text { NFIP } & \text { North Carolina } \\ & \text { National Flood Insurance Program } \\ & \end{array}$


ODD+D Overview, Design Concepts and Details + Human Decision-making PMT Protection Motivation Theory

PT Prospect Theory

RMSE Root Mean Squared prediction Error

RN Risk Negligence

SD Standard deviation

SDE Standard Deviation of prediction Error

SES Social-Ecological/Socio-Environmental Systems

SFHA(s) Special Flood Hazard Area(s)

U.S. $\quad$ United States

WTA Willingness to accept

WTP Willingness to pay

\section{List of Tables}

Table 2.I Summary statistics of the property attributes

Table 2.2 Input variables and their functional forms for the hedonic models

Table 2.3 Stability of a regression coefficient of the floodplain variable over various in-sample sizes

Table 3.I Input variables of the utility function

Table 3.2 Summary statistics for the six models of risk perception. Buyer agents have normal preference for neighbourhood quality: gamma $=$ I

Table 3.3 Summary statistics for the six models of risk perception. Buyer agents have no preference for neighbourhood quality: gamma $=0$

Table 3.4 Summary statistics for the six models of risk perception in the randomised landscape. Buyer agents have normal preference for neighbourhood quality: gamma = I 
Table 4.I Relative importance of property attributes in the agents' utility function

Table 4.2 Summary stats for the flood zone coefficient

Table 4.3 Total housing capital at risk in the 100 -year flood zone

Table 5.I Demographics of survey respondents

Table 5.2 Summary statistics of the variables included in the analysis

Table 5.3 List of explanatory variables and dependent variables included in the stepwise regression.

Table AI Experimental settings - columns 2 and 3 - for each section of the Results chapter. The fourth column indicates which research objective is tackled by each section and the fifth column gives the rationale for the experimental settings.

Table A2 Summary Statistics of the Data $(\mathrm{N}=348 \mathrm{I})$ for Beaufort, NC

Table A3 Prior likelihood of hypothesised effect sizes for all statistical relations in the conceptual model, used to calculate the Bayesian posterior probabilities of the hypotheses.

\section{List of Figures}

Figure I.I Open challenges for modellers how to capture human behaviour in ABM. The three steps 'Conceptualisation', 'Validation', and 'Operationalisation' highlight where the modeller is faced with a choice. The arrows represent a typical pathway of the choices that modellers go through, although they may not necessarily follow this particular order, and dependencies between the three steps do exist.

Figure I.2 Projected property price with flood events whereby flood probability does not change over time. Note the sudden drop after the event and the slow recovery of the price. After Pryce et al. (20II)

Figure 2.I Four out-of-sample prediction performance metrics as function of sample fraction, comparing MI (continuous) and M2 (dashed). In black: smoothing spline of the moving-window median. In grey: the $95 \%$ confidence interval boundary. 
Figure 2.2 Four out-of-sample prediction performance metrics as function of sample fraction, comparing M3 (continuous) and M2 (dashed). In black: smoothing spline of the moving-window median. In grey: the $95 \%$ confidence interval boundary.

Figure 2.3 Semivariogram of the in-sample residuals of M3. The graph shows that there is spatial autocorrelation in the residuals, since the variance in residuals increases with distance between properties.

Figure 2.4 Out-of-sample prediction performance of M3 (continuous) and M4 with kriging (dashed) as function of sample fraction. In black: smoothing spline of the moving-window median. In grey: $95 \%$ confidence interval boundary.

Figure 3.I Discount for flood-prone properties in the last 5 years of the simulations in the real landscape. The models shown are expected utility, risk negligence, prospect theory with no floods as reference point and prospect theory with 3 floods as reference point. Gamma indicates buyer's preference for neighbourhood quality. Error bars indicate one standard deviation, i.e. effect size.

Figure 3.2 Discount for flood-prone properties in the last 5 years of the simulations in the random landscape. The models shown are expected utility, risk negligence, prospect theory with no floods as reference point and prospect theory with 3 floods as reference point. Gamma indicates buyer's preference for neighbourhood quality. Error bars indicate one standard deviation, i.e. effect size.

Figure 4.I Map of the study area. Dots indicate the centroid of a parcel with colors indicating the parcels' flood zone designations: red $=100$ year , orange $=500$-year and green $=$ safe zone.

Figure 4.2 Agent-based housing market model

Figure 4.3 Properties sold in each flood zone, distributed in household income percentiles

Figure 4.4 Discount for Properties in the 100-year (A) and 500-year (B) flood zone

Figure 5.I Impacts and effect sizes (A) of factors that govern buyers' responses to flood risk. B highlights the Bayesian posterior 
likelihood of the effect. Red indicates negative effects and green the positive. Lighter arrows indicate weaker evidence for the effect. We added the mean value of each variable $(\mu)$ and, if applicable, the standard deviation $(\sigma)$ that allow for a more meaningful interpretation of the effect size. Thicker lines indicate a stronger effect.

Figure 5.2 Impacts and effect sizes $(A)$ of factors that govern sellers' responses to flood risk. B highlights the Bayesian posterior likelihood of the effect. Red indicates negative effects and green the positive. Lighter arrows indicate weaker evidence for the effect. We added the mean value of each variable $(\mu)$ and, if applicable, the standard deviation $(\sigma)$ that allow for a more meaningful interpretation of the effect size. Thicker lines indicate a stronger effect. The main dependent variables are highlighted in grey. The box 'moving out of the flood zone' indicates people that sell their property for all sorts of reasons, and choose to live outside the flood zone after the move. The box 'moving out to evade the hazard' is a subset of the former group that do so explicitly to escape the hazard of flooding.

Figure 6.I Schematic representation of interactions between buyers and sellers, and the real estate agent that updates the price expectation based on property transactions in the bilateral housing market model. Source: Filatova 2015

Figure 6.2 Schematic representation of buyer behaviour in response to flood risk. Red indicates negative effects and green the positive. The strength of the impact is given by the thickness of the lines. The main responses of buyer agents in the model are highlighted in grey.

Figure 6.3 Schematic representation of seller behaviour in response to flood risk. Red indicates negative effects and green the positive. The strength of the impact is given by the thickness of the lines. The main responses of seller agents in the model are highlighted in grey. The box 'moving out of the flood zone' indicates household agents that sell their property for all sorts of reasons, and choose to live outside the flood zone after the move. The box 'moving out to evade the hazard' is a subset of the former group highlighting agents sell their house explicitly to escape the hazard of flooding. 
Figure 6.4 Change in property values in the 100-year and 500-year flood zones as a result of flooding. Recovery time differs between the cases because Greenville has a lower proportion of properties in the flood zone. Lines represent the average impact of the first flood (red) and of both floods combined (blue) across 663 Monte Carlo model runs. The bands represent $80 \%$ of the runs.

Figure 6.5 Households that want to move out of the flood zone after the flood but cannot. Either they withdraw their property from the market after a number of unsuccessful attempts due to low demand (Fig 6.5.a and 6.5.b), or because their mortgage debt is higher than the market value of the property (Fig 6.5.c and 6.5.d). Lines represent the average impact of the first flood (red) and of both floods combined (blue) across 663 Monte Carlo model runs. The bands represent $80 \%$ of the runs.

Figure 6.6 Change in poverty in the 100-year and 500-year reoccurring flood zones as a result of flooding. We use the 2016 US poverty threshold for 4-person households as a reference (US Census Bureau 2017). Lines represent the average impact of the first flood (red) and of both floods combined (blue) across 663 Monte Carlo model runs. The bands represent $80 \%$ of the runs.

Figure 7.I Steps to implement human behaviour in models of CHANS. Note that exploring how to improve the model may not necessarily lead to repeating all steps, as is shown by the short-cuts of the dashed arrows.

Figure AI Relationship between house square footage and sales price, including the fitted regressions with squared and log functional forms.

Figure A2 Model parameters converging to an equilibrium in the simulations. The time to reach an equilibrium depends largely on individual preferences for neighbourhood quality (the value of gamma), and somewhat on the behavioural risk perception model. 


\section{SUMMARY}

Humans have a major impact on what the world looks like today. So much so, that we can speak of a new geological epoch: the Anthropocene. Some of the impacts of our collective behaviour are unintended, and yet it is accelerating beyond our control. These impacts include changes that are undesirable, and that may cause conflict and war over natural resources. The challenge for modern humans is to counter the undesirable changes that affect our collective well-being. Changes such as environmental degradation, climate change and rapid loss of global biodiversity. For scientists it implies that we must try to understand the impacts that people have on their environment and vice versa, and consequently how nature and society may change over time. This requires an interdisciplinary approach in which we study nature and society as a whole. We study them in systems referred to as coupled human-and-natural systems (CHANS). Such systems are highly complex, with many elements interacting, learning, adapting, and influencing each other. Hence, it is difficult to trace intuitively how humans influence the dynamics of CHANS over time. Computational models and simulation studies provide a useful tool for studying such dynamics. Agent-Based Modelling (ABM) is one of the tools that is increasingly used for its capability of capturing the complexity of CHANS. However, the complex dynamics of human behaviour and its impact on the environment is still underrepresented in CHANS prediction models. Furthermore, there are many degrees of freedom in terms of how to model human behaviour in ABMs of CHANS, and guidance is necessary to make strategic modelling decisions. In this methodological thesis I address these issues with the following research goal:

Improving the modelling of human behaviour in coupled human and natural systems, in agent-based models that are designed for exploring global environmental change scenarios and policy intervention strategies.

This goal encompasses improving the usability of $A B M s$ beyond understanding systems in the present alone. Our models must be usable to explore changes that can emerge in societies and natural systems in the future as a consequence of the expanding collective impact of human behaviour. I address this methodological challenge in a case study of the housing market in flood-prone areas that are increasingly at risk as a consequence of climate change. Throughout the thesis I make step by step changes to an existing ABM of the housing market that is designed to predict how households will respond to increasing flood risk and how demographics of coastal cities may change as a consequence of that.

In chapter 2 I address how to capture the collective preferences of a variety of individuals in a dynamic environment. This is important because we ultimately need to trace the patterns that emerge in society as a consequence of collective behaviour. Chapter 2 shows how collective preferences can be traced in dynamic situations where preferences change constantly. In these cases we need to make an accurate 
assessment with little available data, given that there is little time to collect data. Although the strength of $A B M$ lies in the detailed modelling a large number of individuals with diverse preferences, capturing the collective preferences of these individuals requires a more abstract and simplified approach. My findings in chapter 2 show that models with fewer variables perform consistently better than models that include as many variables as possible. The reason for that is that the preferences of the collective comprises only those preferences that people have in common. A modeller should therefore only include those variables that represent very general rules-of-thumb.

Chapter 3 and chapter 4 deal with the aggregate impacts of various micro-level behavioural theories and assumptions in an ABM. I assess how the choice of a behavioural theory impacts the patterns in the simulations that emerge on macro scale, and thus how sensitively the patterns in the model respond to different assumptions of micro-level behaviour. In chapter 3 I use historical empirical data to assess which micro-level behavioural theory in the model result in a macro-level outcome that resembles the patterns observed in empirical data. Then, in chapter 4 I use the $A B M$ to assess the effectiveness of a certain policy intervention on the macro scale. This is relevant in case we want to steer the impact of human behaviour in a certain direction through policy intervention. Chapters 3 and 4 illustrate that the model can behave very sensitively to the micro-level behavioural assumptions, the choice of a theory, and even how a theory is formalised and implemented in the model. Furthermore, chapters 3 and 4 highlight a discrepancy between micro- and macro-level measurements of peoples' behavioural preferences driven by all sorts of statistical biases. The simulations of chapter 4 show that the perceived net impact of a policy intervention is highly sensitive to behavioural assumptions on the microlevel. Therefore, if we want to use an ABM to explore which interventions steer human behaviour in CHANS in which direction, we cannot rely on a model where the micro-level foundations are parametrised on macro-level data.

In chapter 5 I use more detailed micro-level empirical data rather than macro level data. Naturally, an increased level of detail leads to more realistic representation of human behaviour in ABM. Yet, a more realistic representation of human behaviour may also result in redundant complexity of our models. Chapter 5 highlights that validation of human behaviour with empirical data has a clear added value when selecting behavioural theories for an ABM. This is important to be aware of as a modeller. Even theory-based ABMs need to be parametrised somehow to formalise decision rules in a model, and empirical data can be instrumental in doing so. Furthermore, in chapter 5 I look at the importance of context in the decisions of households, and I look at which theories fit better in which context. I use interview and survey data to understand human behaviour better in the specific decision contexts that I try to describe in the ABM. In my results I find that people behave 
differently in different contexts, and that the context determines which behavioural theory fits best in the model.

In chapter 6 I put the validated model to the test. I simulate the emergence of regime shifts from the bottom up, when changes in the natural system affect changes in human behaviour. I scale up the micro-level behaviour that I observed in empirical data and extrapolate it into the future of a changing climate. It is expected that global environmental change such as climate change will cause changing human behaviour, and consequently it may cause structural shifts in the state of CHANS. These structural shifts are demonstrated in the simulation study in chapter 6 . The simulation study can be used to explore what kind of macro-level output may emerge in an $A B M$ through micro-level behavioural triggers. Although we do not have empirical data to validate these predictions, one can qualitatively assess whether the predictions of the model present a plausible outcome, given our confidence in the model design and our understanding of the dynamics in the system. Moreover, chapter 6 shows the complexity of the output of an ABM and the benefits thereof. In chapter $6 \mathrm{I}$ show that different patterns emerge in a number of macro level variables. These variables are relevant indicators of the system's functioning and outlook. The diversity of these patterns and the diversity of conclusions that are drawn if they are assessed separately indicates why $A B M$ does justice to the complexity of CHANS more than any other modelling technique.

The findings of my thesis contribute to current modelling practices in 3 ways. First, my thesis contributes to finding the sweet spot between complicatedness and simplicity in modelling CHANS with ABMs. This is a much debated issue in the field of ABM. The contribution of my thesis is that I show on specific levels of the model (micro-macro) where the model needs to be simplified as much as possible and where it needs more complicated detail. On one hand I see that simplifying the model as much as possible on the macro level produces better predictions. Contrastingly on the micro level I make improvements to the model by making it more complicated: adding heterogeneity to the agents, adding more complex theories of bounded rational behaviour, and adding more micro-level empirical data to inform behavioural rules. Secondly, my thesis contributes to the question what kind of data to use and which behavioural theories to select in order to formulate human behaviour in our models. In my results I find that the theories describing human behaviour are highly context-dependent, and therefore a modeller must always understand the context before collecting theories that may accurately describe human behaviour in the context of the model. Moreover, human behaviour in ABMs is ideally always informed by some form of empirical data. Micro-level data such as interviews and surveys are preferred over macro-level data, because it adds more detail on the individual actors. This does justice to the complex individual-oriented approach of ABM. Thirdly, I propose a number of steps that every modeller should undertake when trying to improve the formalisation of human behaviour in $A B M s$ of 
Summary

CHANS. These are: (I) build a conceptual model of the system, (2) select a number of theories on human behaviour that may fit in the context, (3) collect empirical data to support the choice of a theory, (4) implement the behavioural rules in the model and start exploring the model predictions, (5) perform sensitivity analyses, (6) interpret the model output, and (7) explore how the model can be improved. The model may continuously be improved after the last step, not necessarily starting from step I. 


\section{SAMENVATTING}

Mensen hebben een enorme invloed op hoe de wereld er vandaag de dag uitziet. Zo veel dat we kunnen spreken van een nieuw geologisch tijdperk: het Antropoceen. Sommige effecten van ons collectief gedrag zijn onbedoeld, en toch verliezen we er snel de controle over. Onder deze effecten zijn veranderingen die onwenselijk zijn, en die zorgen voor conflict en oorlog over natuurlijke hulpbronnen. De uitdaging voor de moderne mens is het tegengaan van de onwenselijke veranderingen die ons collectief welzijn aangaan. Veranderingen zoals milieudegradatie, klimaatverandering en een snel verlies van wereldwijde biodiversiteit. Voor wetenschappers betekent dit dat we moeten proberen te begrijpen wat voor invloed mensen op hun omgeving hebben en vice versa, en uiteindelijk hoe natuur en maatschappij met de tijd kunnen veranderen. Hiervoor is een interdisciplinaire aanpak nodig waarbij we natuur en maatschappij als één geheel bestuderen. We bestuderen ze in systemen die we 'gekoppelde mens-natuur-systemen' (CHANS) noemen. Dit soort systemen zijn zeer complex, met veel interacterende elementen die leren, zich aanpassen en elkaar beïnvloeden. Vandaar dat het moeilijk is om intuïtief te traceren hoe mensen de dynamiek van CHANS over de tijd beïnvloeden. Wiskundig modellen en simulatiestudies zijn een goede manier om dit soort dynamiek te bestuderen. Agentbased modelleren (ABM) is een van de tools die steeds meer wordt gebruikt vanwege de mogelijkheid om de complexiteit van CHANS te omvatten. De complexiteit van menselijk gedrag en zijn invloed op zijn omgeving is echter nog steeds ondervertegenwoordigd in voorspellingsmodellen van CHANS. Verder zijne er vele manieren waarop menselijk gedrag in ABM's van CHANS gemodelleerd kan worden. Daarom is sturing nodig om strategische beslissingen te nemen in het modelleren. In deze methodologische thesis belicht ik deze issues met het volgende onderzoeksdoel:

Verbeteren hoe menselijk gedrag gemodelleerd wordt in gekoppelde mens-natuur-systemen, in agent-based modellen die gemaakt zijn om wereldwijde milieuverandering scenario's en beleidsinterventiestrategieën te verkennen.

Dit doel omvat het verbeteren van de bruikbaarheid van ABM's die verder gaat dan het begrijpen van systemen in het heden alleen. Onze modellen moeten bruikbaar zijn om veranderingen te onderzoeken die zich in de toekomst kunnen voordoen in samenlevingen en natuurlijke systemen als gevolg van de groeiende impact van collectief menselijk gedrag. Ik ga in op deze methodologische uitdaging in een casestudy van de woningmarkt in overstromingsgevoelige gebieden die steeds meer gevaar lopen als gevolg van klimaatverandering. In mijn proefschrift maak ik stap voor 
stap aanpassingen aan een bestaand $A B M$ van de woningmarkt dat is ontworpen om te voorspellen hoe huishoudens zullen reageren op het toenemende overstromingsrisico en hoe de demografie in kuststeden als gevolg daarvan kan veranderen.

In hoofdstuk 2 bespreek ik hoe de collectieve voorkeuren van een verscheidenheid aan individuen in een dynamische omgeving kunnen worden vastgelegd. Dit is belangrijk omdat we uiteindelijk de patronen moeten traceren die in de samenleving ontstaan als gevolg van collectief gedrag. Hoofdstuk 2 laat zien hoe collectieve voorkeuren getraceerd kunnen worden in dynamische situaties waarin voorkeuren voortdurend veranderen. In deze gevallen moeten we met weinig beschikbare gegevens een nauwkeurige inschatting maken, aangezien er weinig tijd is om gegevens te verzamelen. De kracht van ABM ligt in het gedetailleerd modelleren van een groot aantal individuen met uiteenlopende voorkeuren. Toch vereist het vastleggen van de overeenkomstige voorkeuren van deze individuen een meer abstracte en vereenvoudigde aanpak. Mijn bevindingen in hoofdstuk 2 laten zien dat modellen met minder variabelen consistent beter presteren dan modellen die zoveel mogelijk variabelen bevatten. De reden hiervoor is dat het alleen die voorkeuren betreft die mensen gemeen hebben binnen het collectief. Een modelbouwer zou daarom alleen die variabelen moeten opnemen die zeer algemene vuistregels vertegenwoordigen.

Hoofdstuk 3 en hoofdstuk 4 gaan in op de geaggregeerde impact van verschillende gedragstheorieën en -aannames in een ABM. Ik beoordeel hoe de keuze van een gedragstheorie invloed heeft op de patronen in de simulaties die op macroschaal ontstaan, en dus hoe gevoelig de patronen in het model reageren op verschillende aannames over gedrag op microniveau. In hoofdstuk 3 gebruik ik historische gegevens om te beoordelen welke gedragstheorie in het model resulteert in een patroon op macroschaal dat lijkt op de patronen die in empirische data zijn waargenomen. Vervolgens gebruik ik in hoofdstuk 4 het ABM om de effectiviteit van een bepaalde beleidsinterventie op macroschaal te beoordelen. Dit is relevant wanneer we de impact van menselijk gedrag door beleidsinterventie in een bepaalde richting willen sturen. De hoofdstukken 3 en 4 illustreren dat het model zeer gevoelig kan reageren op de verschillende gedragsaannames, op de keuze van een gedragstheorie, en zelfs op de manier waarop een gedragstheorie wordt geïmplementeerd in het model. Bovendien benadrukken de hoofdstukken 3 en 4 een discrepantie tussen micro- en macroniveau metingen van de gedragsvoorkeuren van mensen, als gevolg van allerlei statistische vertekeningen. De simulaties van hoofdstuk 4 tonen aan dat de verwachte netto-impact van een beleidsinterventie zeer gevoelig is voor aannames van gedrag op microniveau. Als we een ABM willen gebruiken om te onderzoeken welke 
interventies het menselijk gedrag in welke richting sturen in CHANS, kunnen we niet vertrouwen op een model waarbij de aannames op microniveau geparametriseerd worden op basis van geaggregeerde data.

In hoofdstuk 5 gebruik ik meer gedetailleerde empirische gegevens op microniveau in plaats van op macroniveau. Vanzelfsprekend leidt een verhoogd detailniveau tot een meer realistische weergave van menselijk gedrag in ABM. Toch kan een meer realistische weergave van menselijk gedrag ook leiden tot onnodig ingewikkelde modellen. Hoofdstuk 5 benadrukt dat voor validatie van menselijk gedrag empirische gegevens duidelijk een toegevoegde waarde hebben bij het selecteren van gedragstheorieën voor een ABM. Het is belangrijk om je hier als modelbouwer bewust van te zijn. Zelfs theoretische ABM's moeten op de een of andere manier geparametriseerd worden om gedragsregels in een model te formaliseren, en empirische gegevens kunnen hierbij een uitkomst bieden. Verder kijk ik in hoofdstuk 5 naar het belang van context in de beslissingen van huishoudens. Ik kijk naar welke gedragstheorieën beter in welke context passen. Ik maak gebruik van interviews en enquêtes om het menselijk gedrag beter te begrijpen in de specifieke beslissingscontexten die ik in het ABM probeer te beschrijven. In mijn resultaten vind $i k$ dat mensen zich in verschillende contexten anders gedragen en dat de context bepalend is voor welke gedragstheorie het beste past in het model.

In hoofdstuk 6 stel ik het gevalideerde model op de proef. lk simuleer het ontstaan van regimeverschuivingen van onderop, wanneer het menselijk gedrag verandert door veranderingen in het milieu. Ik schaal het micro-gedrag dat ik in empirische gegevens heb waargenomen op en extrapoleer het naar de toekomst waarin ons klimaat zal veranderen. Verwacht wordt dat wereldwijde milieuveranderingen, zoals klimaatverandering, zullen leiden tot veranderend menselijk gedrag, en dus tot structurele verschuivingen in de toestand van CHANS. Deze structurele verschuivingen worden gedemonstreerd in de simulatiestudie in hoofdstuk 6. De simulatiestudie kan worden gebruikt om te verkennen welk soort macroniveauoutput kan ontstaan in een $A B M$ als gevolg van gedragstriggers op microniveau. Hoewel we geen empirische gegevens hebben om deze voorspellingen te valideren, kan men kwalitatief beoordelen of de voorspellingen van het model een plausibel resultaat opleveren. Dit is mede afhankelijk van ons vertrouwen in het modelontwerp en ons begrip van de dynamiek in het systeem. Bovendien toont hoofdstuk 6 de complexiteit van de output van een ABM en de voordelen daarvan. In hoofdstuk 6 laat ik zien dat er verschillende patronen naar voren komen in een aantal variabelen op macroniveau. Dit betreft variabelen die relevant zijn als indicator voor het systeem qua uiterlijk en functioneren ervan. De diversiteit van deze 
patronen en de diversiteit van de conclusies die worden getrokken als ze afzonderlijk worden beoordeeld, geeft aan waarom ABM meer recht doet aan de complexiteit van CHANS dan enige andere modelleertechniek.

De bevindingen van mijn proefschrift dragen op 3 manieren bij aan de huidige modelleerpraktijk. Ten eerste draagt mijn scriptie bij aan de zoektocht naar de juiste balans tussen complexiteit en eenvoud in het modelleren van CHANS met ABM's. Dit is een veelbesproken onderwerp in het veld van $A B M$. De bijdrage van mijn scriptie is dat ik op specifieke niveaus van het model (micromacro) laat zien waar het model zo veel mogelijk vereenvoudigd moet worden en waar complexere details nodig zijn. Aan de ene kant zie ik dat het zoveel mogelijk vereenvoudigen van het model op macroniveau betere voorspellingen oplevert. Contrasterend breng ik op microniveau verbeteringen aan door het model ingewikkelder te maken: het toevoegen van heterogeniteit van de agenten, het toevoegen van complexere theorieën over beperkt rationeel gedrag, en het toevoegen van meer empirische data op microniveau om gedragsregels te van af te leiden. Ten tweede draagt mijn proefschrift bij aan de vraag wat voor validatiedata te gebruiken en welke gedragstheorieën te selecteren om menselijk gedrag in onze modellen te formuleren. In mijn resultaten vind ik dat de theorieën die het menselijk gedrag beschrijven in hoge mate contextafhankelijk zijn, en daarom moet een modelbouwer altijd de context begrijpen voordat hij theorieën verzamelt die het menselijk gedrag beschrijven in de juiste context van het model. Bovendien is het menselijk gedrag in ABM's idealiter altijd gebaseerd op empirische data in één of andere vorm. Gegevens op microniveau, zoals interviews en enquêtes, hebben de voorkeur boven gegevens op macroniveau, omdat het meer details over de individuele actoren toevoegt. Dit doet recht aan de complexe individueel georiënteerde benadering van ABM. Ten derde stel ik een aantal stappen voor die elke modelbouwer zou moeten ondernemen om de formalisering van menselijk gedrag in ABM's van CHANS te verbeteren. Deze zijn: (I) bouw een conceptueel model van het system, (2) selecteer een aantal theorieën over menselijk gedrag die in de context passen, (3) verzamel empirische gegevens om de keuze van een theorie te ondersteunen, (4) implementeer de gedragsregels in het model en begin de modelvoorspellingen te verkennen, (5) voer een sensitiviteitsanalyses uit, (6) interpreteer de modeloutput, en (7) onderzoek hoe het model kan worden verbeterd. Het model kan na de laatste stap continu worden verbeterd, niet noodzakelijk vanaf stap I. 


\section{About the author}

Koen de Koning was born in Venlo, the Netherlands, on the $25^{\text {th }}$ of July 1991. He went to primary school at Basischool de Springbeek in Hout-Blerick. After that he went to high school at Blariacumcollege Venlo. He graduated high school in 2009.

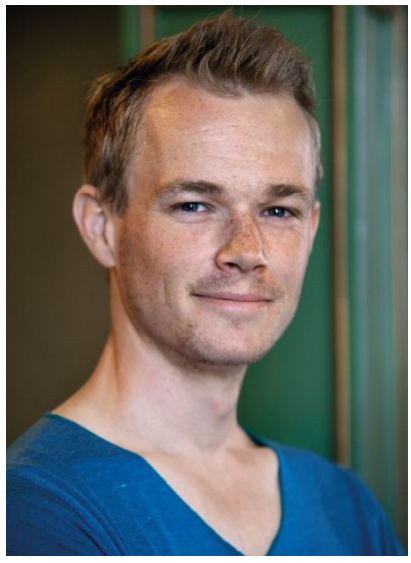

In 2009 he started the bachelor's programme of Bos- en Natuurbeheer (English: Forest and Nature Conservation) at Wageningen University. During his bachelor's he did the specialisation of Ecology and Management. He did his thesis on facilitation between herbivorous mammals. In 2012 he started the master's programme Forest and Nature Conservation at Wageningen University. He specialised his master's in ecological modelling. In 2014 he did his thesis on the movement patterns of African grazers: wildebeest and zebra. He spent three months on the South African savannah for his field work. In the summer of 2014 he did a fourmonth internship at the department of Natural Resource Management, faculty of ITC, University of Twente. During his internship he worked on time series of satellite images to assess the stability of forest ecosystems in Australia. Koen graduated his MSc in 2014 with the honorary distinction 'Cum Laude'.

Koen started his $\mathrm{PhD}$ in January 2015 at the department of Governance and Technology for Sustainability (CSTM), faculty of BMS, University of Twente. In 2015 he joined the IGS PhD Initiative Group and the PhD Network of the University of Twente (P-NUT). Since that time he helped in organising several PhD networking events and social events. In 2016 he fulfilled the role as treasurer of P-NUT, and in 2017 he fulfilled the role of president. In 2018, Koen was awarded as one of the 'Faces-of-Science' by the Royal Dutch Academy of Sciences (KNAW). Since that time he has been blogging about his life as a researcher on the website of NEMO Kennislink. 


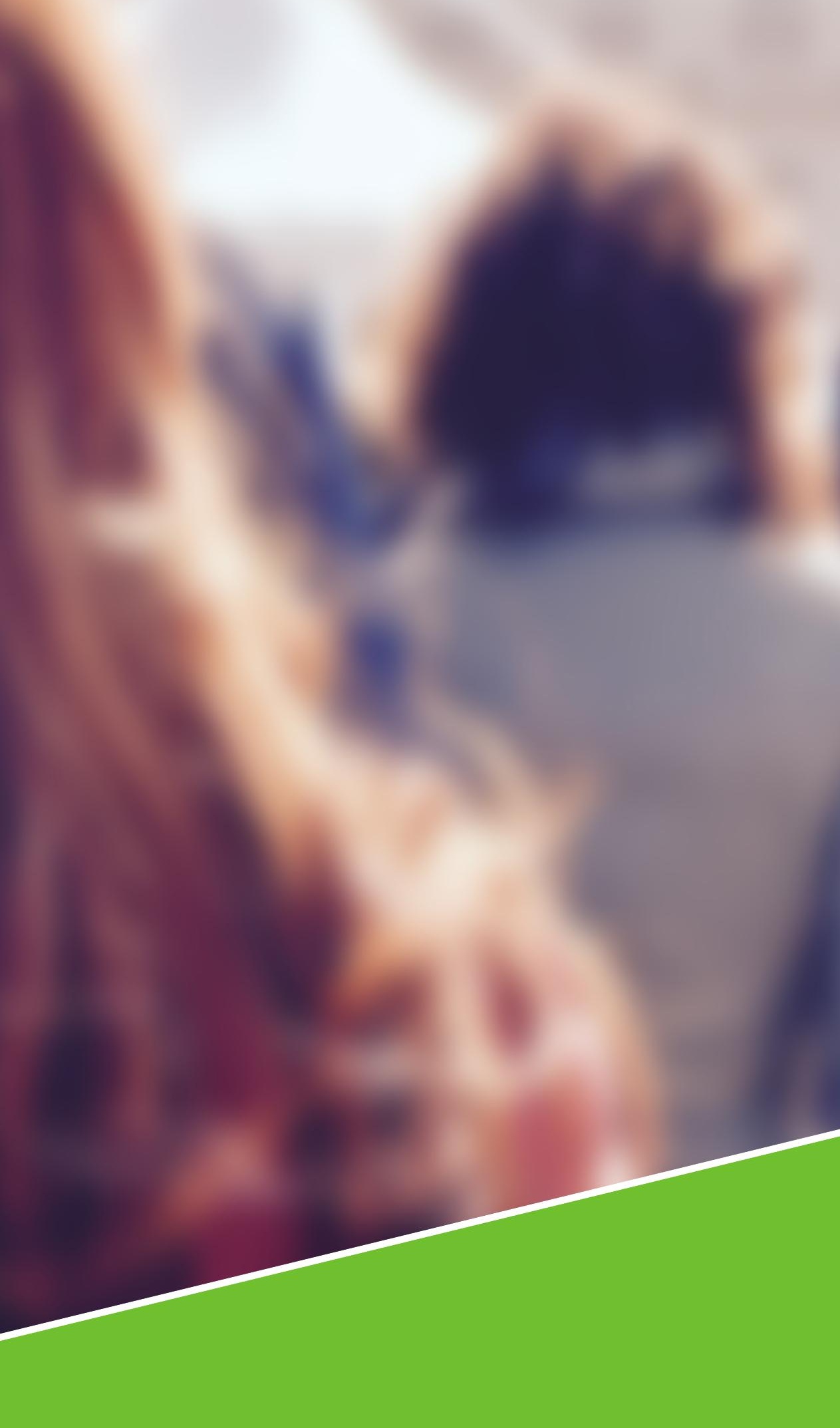

